\title{
Prepartal overfeeding alters the lipidomic profiles in the liver and the adipose tissue of transition dairy cows
}

\section{Qin, Nanbing}

2017-02

Qin , N , Kokkonen , T , Salin , S , Seppänen-Laakso , T , Taponen , J , Vanhatalo , A \& Elo , $\mathrm{K} 2017$, ' Prepartal overfeeding alters the lipidomic profiles in the liver and the adipose tissue of transition dairy cows ' , Metabolomics , vol. 13 , no. 2 , 21 . https://doi.org/10.1007/s11306-016-1160-0

http://hdl.handle.net/10138/309504

https://doi.org/10.1007/s11306-016-1160-0

unspecified

acceptedVersion

Downloaded from Helda, University of Helsinki institutional repository.

This is an electronic reprint of the original article.

This reprint may differ from the original in pagination and typographic detail.

Please cite the original version. 


\section{Abstract}

Introduction

Physiological adaptations in the energy metabolism of dairy cows during the periparturient period are partly mediated by insulin resistance (IR), which may subsequently induce metabolic disorders postpartum. The molecular mechanisms underlying IR in dairy cows are largely unknown.

Objective

This study aimed to find a novel insight into the molecular mechanisms underlying IR in dairy cows during the periparturient period by analyzing the effects of prepartal overfeeding on the lipidomic profiles in the liver and adipose tissue (AT).

\section{Methods}

Sixteen cows were allocated to controlled-energy and high-energy feeding groups. Lipidomic profiling was conducted on liver and adipose tissue samples collected at $8 \mathrm{~d}$ prior to the predicted parturition, and $1 \mathrm{~d}$ (only AT) and $9 \mathrm{~d}$ after the actual parturition.

Results

Five ceramides (Cers) were identified to be significantly increased by prepartal overfeeding in AT before parturition. Principal component-linear discriminant analysis showed that the differences in lipidomic profiles between the feeding groups were mainly characterized by phosphatidylcholines (PC), phosphatidylethanolamines (PE), lysophophosphatidylcholines (LysoPC), and lysophosphatidylethanolamines (LysoPE) in the liver, and by Cer, PE, and phosphatidylinositols (PI) in AT. Lipid class levels indicated that prepartal overfeeding elevated the concentration of PE, PI, LysoPC, LysoPE, and sphingomyelin (SM) in the liver, and increased the concentration of Cer in AT during the periparturient period.

\section{Conclusion}

Prepartal overfeeding significantly altered the concentrations of various sphingolipids, phospholipids, and lysophospholipids in the liver and AT of dairy cows during the periparturient period.

Key words: energy metabolism, molecular adaptation, altered lipid profiles, transition dairy cow, ceramides 
Dairy cows suffer from negative energy balance during early lactation, which results from a combination of the increasing glucose requirement in lactating mammary glands and insufficient dry matter intake (DMI) (Bertics et al. 1992). A series of physiological adaptions are mediated by insulin resistance (IR) to allocate the glucose, amino acids, and fatty acids to the tissue in demand in periparturient dairy cows (Bell and Bauman 1997). The development of IR induces augmented hepatic gluconeogenesis, hepatic glycogenolysis, protein mobilization from skeletal muscle, and lipolysis in the adipose tissue (AT) (De Koster and Opsomer 2013; Hayirli 2006). Although these alterations in the energy metabolism are usually physiological responses that affect the supply of the nutrients and energy that are increasingly required by the fetus and by the onset of lactation (Coppock 1985), the response also increases the risk of metabolic disorders in the postparturient cows (Ingvartsen 2006). These metabolic disorders include hepatic lipidosis and ketosis induced by the excessive mobilization of non-esterified fatty acids (NEFA) from the AT into the liver (Drackley et al. 1992; Reid 1980; Veenhuizen et al. 1991),

Prepartal nutritional strategies affect the postpartal health of dairy cows as the periparturient physiological adaptions are related to the change in energy balance that occurs near calving (Douglas et al. 2006). Prepartal oversupply of energy decreases lipogenesis and increases lipolysis in AT postpartum when adiposity of the cows is increased (Nielsen et al. 2010). As a result, exacerbated adipose mobilization was observed postpartum in overfed cows, characterized by higher NEFA levels compared to the cows fed according to the requirement (Rukkwamsuk et al. 1998). The NEFA delivered to the liver promote hepatic lipogenesis through the activation of peroxisome proliferator-activated receptor alpha (PPAR $\alpha$ ) (Ji et al. 2012; Khan et al. 2014). In addition, prepartal overfeeding decreases fatty acid oxidation capacity of the liver (Selim et al. 2014). The combination of these changes potentially lead to the accumulation of triglycerides in the liver (Drackley et al. 2005; Loor et al. 2006). These changes in lipid metabolism might be mediated by lower whole body insulin sensitivity caused by prepartal overfeeding (Holtenius et al. 2003).

The molecular mechanisms of IR have been studied extensively in humans and rodents (Chavez and Summers 2010; Erion and Shulman 2010; McArdle et al. 2013). It is widely accepted that IR is closely associated with obesity and induced by lipids (Kahn and Flier 2000). Specifically, ceramides and diacylglycerols are the two lipid classes that serve as potential triggers of IR in human and mice. Ceramides block phosphoinositide 3-kinase insulin signaling pathway by interfering the translocation of protein kinase B (Akt/PKB) (Chavez and Summers 2010). Diacylglycerols interfere the phosphorylation of insulin receptor substrate 1 and 2 (IRS1 and IRS2) through the activation of protein kinase C (PKC) (Erion and Shulman 2010). In addition, sphingomyelins, phosphatidylcholines, phosphatidylethanolamines, phosphatidylinositols, phosphatidylserines, lysophophosphatidylcholines, lysophosphatidylethanolamines, and cholesterol esters were identified as the biomarkers of IR as their concentrations were found to differ between healthy individuals and insulin-resistant individuals (Haus et al. 2009; Rauschert et al. 2016; Wallace et al. 2014). Moreover, some lipid subspecies were identified to be associated with alterations in nutritional intake levels and the obesity-related diseases (Eisinger et al. 2014a; Hanamatsu et al. 2014; Yetukuri et al. 2007).

In the past few years, lipidomic studies on plasma lipid compositions of periparturient dairy cows have revealed associations between the changes in lipid profiles and the physiological adaptations. Imhasly et al. (2014 and 2015) reported that the concentration of triglycerides, phosphatidylcholines, lysophophosphatidylcholines, and sphingomyelins 
in the plasma of dairy cows changed significantly from $14 \mathrm{~d}$ prepartum to $28 \mathrm{~d}$ postpartum. The same authors identified that the concentrations of six phosphatidylcholine subspecies in the plasma were highly correlated with the occurrence of hepatic lipidosis (Imhasly et al. 2014). In addition, sphingolipids including ceramides, monohexosylceramides, and lactosylceramides, were found to be elevated in the plasma with the increased adiposity in cows (Rico et al. 2015).

Although analyses of gene expression in various tissues and lipidomic profiling of plasma have been conducted in the investigation of energy metabolism in dairy cows, the molecular mechanisms of IR during the periparturient period at tissue level has remained largely unknown. The main aim of the present study was to analyze the effects of prepartal overfeeding on the lipidomic profiles in the liver and in the AT in dairy cows. It was hypothesized that prepartal dietary energy level significantly affects the concentrations of hepatic and AT lipid subspecies that are potentially associated with IR.

\section{Materials and Methods}

2.1 Animals, diets, experimental design and sampling

Feeding experiment, feed composition, and the collection of biopsy samples were described in detail in Selim et al. (2014). The feeding experiment involved 16 Finnish Ayrshire dairy cows in a randomized complete block design. The cows were paired according to their parity, body weight, and body condition score. The members of each pair were randomly allocated to one of two dietary treatments groups at $44 \pm 5$ days (mean $\pm \mathrm{SD}$ ) prior to the actual parturition date, thus one individual of each pair received the controlled-energy treatment and its partner received the high-energy intake treatment. The grass silage-based dietary treatments were controlled-energy diet (CON; 100\% of energy requirement of pregnant dairy cows) and initially an ad libitum high-energy diet (HIGH) for three weeks. The actual mean energy intake of the HIGH group was initially $142 \%$ of energy requirement of pregnant dairy cows during the first three weeks of the experimental feeding. During the last three weeks before the predicted parturition the energy allowance of the HIGH group was sequentially decreased in decrements of $5 \%$ on alternate days. The average metabolizable energy (ME) was $99 \mathrm{MJ} / \mathrm{d}$ in CON group and $141 \mathrm{MJ} / \mathrm{d}$ in HIGH group from week 6 prepartum to week 4 prepartum and $109 \mathrm{MJ} / \mathrm{d}$ in CON group and $128 \mathrm{MJ} / \mathrm{d}$ in HIGH group from week 3 prepartum to week 1 prepartum. The data on measurements of feed intake, dry mater intake, milk yield live weight, body condition score (BCS), blood concentrations of glucose, NEFA, beta-hydroxybutyrate, glucagon, and insulin during the periparturient period were previously published by Selim et al. (2014).

Subcutaneous AT samples were collected by biopsy $8 \mathrm{~d}$ before the predicted parturition ( $11 \pm 5 \mathrm{~d}$ in the actual operation) and 1 and $9( \pm 1)$ d postpartum (the three time points are represented as $-8 \mathrm{~d}, 1 \mathrm{~d}$, and $9 \mathrm{~d}$ in the following paragraphs in this paper). The liver biopsies were conducted immediately after the AT biopsies at $-8 \mathrm{~d}$ and $9 \mathrm{~d}$. Liver samples were successfully collected from 13 cows at $-8 \mathrm{~d}$ and from 11 cows at $9 \mathrm{~d}$. AT samples were successfully collected from 15 cows at $-8 \mathrm{~d}$, from 12 cows at $1 \mathrm{~d}$, and from 13 cows at $9 \mathrm{~d}$, respectively. The data of the liver samples of two cows were finally removed due to the health problems (sciatic nerve paralysis and prolonged inappetence) in these cows after the parturition.

\subsection{Lipidomic profiling}


The liver biopses were pulverized (homogenized) as frozen (fast freezed in dry ice for 1 min) by using a Covaris CryoPrep System (Woburn, Massachusetts, USA). The homogenates were transferred from TissueTubesTM into cryo tubes for further storage at $-80^{\circ} \mathrm{C}$. Typically, $5 \mathrm{mg}$ aliquots of homogenized samples were weighed for lipidomics analyses. Homogenized tissue samples were spiked with an internal standard mixture $(20 \mu 1)$ consisting of lysophosphatidylcholine [LPC(17:0)], ceramide [Cer(d18:1/17:0)], phosphatidylcholine [PC(17:0/17:0)], phosphatidyl-ethanolamine [PE(17:0/17:0)] and triacylglycerol [TG(17:0/17:0/17:0], at concentration levels that varied from 1.08 to $2.12 \mu \mathrm{g}$. Sodium chloride solution $(15 \mathrm{mM}, 50 \mu \mathrm{l})$ was added to the samples and lipids that were extracted with a mixture of chloroform and methanol $(2: 1,400 \mu \mathrm{l})$ by using a Retsch Mixer Mill homogenizer (Retsch $\mathrm{GmbH}$, Haan, Germany) at $-20^{\circ} \mathrm{C}$ for 2 $\min (20 \mathrm{~Hz}, 2$ grinding balls $\emptyset 3 \mathrm{~mm})$. After 30-60 min extraction time at room temperature, the samples were centrifuged (Eppendorf 5804R) at $10620 \mathrm{~g}$ for $3 \mathrm{~min}$. A $200 \mu \mathrm{l}$ quantity was taken from the separated lower organic solvent layer and transferred into a vial insert and mixed with $10 \mu \mathrm{l}$ of a standard mixture containing 3 labelled lipid species: L- $\alpha$ lysophosphatidylcholine palmitoyl-D3 (methyl-D3), 1,2-dipalmitoyl-D6-3-sn-glycerophosphatidylcholine (dimethyl-D6) and tripalmitin-1,1,1-13C3 (concentration level $1.0 \mu \mathrm{g}$ ).

Lipidomic analyses were performed on a Waters Q-Tof Premier mass spectrometer combined with an Acquity Ultra Performance LC $^{\mathrm{TM}}$ (UPLC) following the analytical procedure modified from Nygren et al. (2011). All solvents used were LC-MS grade, and the reference lipids were obtained from either Avanti Polar Lipids, Inc. (Alabama, USA) or Larodan Fine Chemicals (Solna, Sweden). Details of the analytical procedure were described in the supplementary material (Online Resource 1).

The lipidomics data were processed by using MZmine 2 software (mzmine.sourceforge.net) including alignment of peaks, peak integration, normalization, and peak identification. Lipids were identified using internal MS and MS-MS spectral library. Quantification of lipid subspecies was based on the peak heights of internal standards. All monoacyl lipids except cholesteryl esters, such as monoacylglycerols and monoacylglycerophospholipids, were normalized by using LPC(17:0), all diacyl lipids except ethanolamine phospholipids were normalized by using PC(17:0/17:0), all ceramides using Cer(d18:1/17:0), all diacyl ethanolamine phospholipids by using PE(17:0/17:0), and TGs and cholesteryl esters by using TG(17:0/17:0/17:0). Other molecular species (including unidentified species) were normalized by using LPC(17:0) for retention time $<300 \mathrm{~s}, \mathrm{PC}(17: 0 / 17: 0)$ for retention time between $300 \mathrm{~s}$ and $410 \mathrm{~s}$, and TG(17:0/17:0/17:0) for higher retention times.

In total, from the liver samples 296 and 1325 masses were detected in the positive electrospray ionization mode (ESI+) and negative electrospray ionization mode (ESI-), respectively, and from the AT samples 541 and 603 masses were detected in ESI+ and ESI-, respectively (Online Resource Table S1). The lipids were identified based on an internal spectral library and were classified into ceramides (Cers), hexosylceramides (HexCers), sphingomyelins (SMs), triacylglycerols (TGs), diacylglycerols (DGs), cholesteryl esters (ChoEs), lysophophosphatidylcholines (LysoPCs), lysophosphatidylethanolamines (LysoPEs), phosphatidylcholines (PCs), phosphatidylethanolamines (PEs), and phosphatidylinositols (PIs). However, a substantial proportion of the detected masses remained unknown.

2.3 Calculations and statistical analyses

2.3.1 Statistical analyses within time points and FDR-calculation 
The statistical analyses were performed using SAS software (release 9.3; SAS Institute, Cary, NC). The concentrations of all detected masses were log2-transformed to normalize the data. Subsequently, Analysis of Variance (ANOVA) between the feeding groups were performed on each variable within each sampling time point by using the PROC MIXED procedure. The model included diet as a fixed effect and block as a random effect. The normalities of all data were tested using the UNIVARIATE procedure based on the residuals of the fitted model. The $P$-values obtained from PROC MIXED procedure were adjusted by the PROC MULTTEST procedure with the AFDR option for the false discovery rate (FDR) calculation (Benjamini and Hochberg 2000). After the FDR threshold calculation was performed, the lipid subspecies were sorted in ascending order using the adjusted $P$-values. The threshold for the FDR indicates the percentage of false positives among the variables with adjusted- $P$ lower than the threshold. In the 4 dataset from liver and AT, the thresholds of adjusted $P$-values were not determined as fixed values, but as the $P$-value of the specific detected mass that produced less than one false positive.

\subsubsection{Principal component analysis-linear discriminant analysis of identified lipids in the liver and AT}

A principal component analysis-linear discriminant analysis model (PCA-LDA) described by Fearn (2008) was applied and the log2-transformed concentrations of all the identified lipid subspecies for both ESI+ and ESI- for the liver and AT as the input was used. This entailed PCA-LDA for 262 subspecies from the liver ESI+, 65 subspecies from the liver ESI-, 55 subspecies from the AT ESI+, and 53 subspecies from the AT ESI- datasets. The subjects in the liver dataset were divided into four groups according to the experimental design and designated: CON $-8 \mathrm{~d}$, CON $9 \mathrm{~d}$, HIGH $-8 \mathrm{~d}$, and HIGH $9 \mathrm{~d}$. The data of the AT dataset were divided into six groups: CON $-8 \mathrm{~d}$, CON $1 \mathrm{~d}$, CON $9 \mathrm{~d}$, HIGH $-8 \mathrm{~d}$, HIGH $1 \mathrm{~d}$, and HIGH 9 d. The PCA scores of all the variables were computed using the PROC PRINCOMP procedure in SAS and used as the input for LDA. The LDA process was iteratively repeated with increasing principal components as the input, starting from 2 . The lowest number of principal components that contributed to $100 \%$ accurate classification in LDA were selected as the final input of LDA. The numbers of the principal components used in the LDA of the liver ESI+, the liver ESI-, AT ESI+, and AT ESI- were calculated to be 8, 10, 27, and 26, respectively. The LDA resulted in 3 PCA-LDA functions (PL functions) that accounted for $100 \%$ of the variation between the four groups in the two liver datasets and five PL functions that accounted for $100 \%$ of variation between six groups in the two AT datasets. The equation of these PL functions can be retrieved in the supplementary materials (Online Resource Table S2).

The PL loadings obtained from the analyses reflected the correlation between the variables and the PL functions. Larger PL loadings indicated greater contributions of the variables to the variation between groups. After PCA-LDA, the variables were ranked by their contribution to the variation between groups caused by diet or time effects according to their corresponding PCA-LDA loadings.

\subsubsection{Repeated measures ANOVA for identified lipid subspecies and the total concentrations of lipid classes}

The total concentration of each lipid class was only calculated for the ESI mode which gave a higher number of detected subspecies. The total concentrations of SMs, TGs, DGs, LysoPCs, LysoPEs, PCs, PEs, and PIs in the liver datasets were calculated from ESI+ detection, whereas the concentrations of Cers and HexCers were calculated from ESI- detection. The total concentrations of ChoEs, SMs, TGs, LysoPCs, and LysoPEs in the AT datasets were calculated from ESI+ detection, whereas those of Cers, HexCers, PCs, PEs, and PIs were calculated from the ESI- detection. 
The concentrations of lipid subspecies and the total concentrations of lipid classes were log-2 transformed to normalize the data prior to the statistical analyses. The preliminary analysis of transformed data was conducted using PROC MIXED procedure with a model including diet as a fixed effect and block as a random effect. The normality of the residuals was checked using PROC UNIVARIATE procedure. The box-and-whisker plots were used to detect the residuals that were not normally distributed.using a model including diet as a fixed effect and block as a random effect. The box-and-whisker plots were composed for the residuals that were not normally distributed. All the observations showing residuals out of the whiskers range in plots could be considered as outliers. Then the normality of the residuals of these variables were repeatedly tested after the most deviated residual from the mean was removed until the normal distribution of the residuals was achieved. Among the total concentrations of the lipid classes, two Cer observations (one at $1 \mathrm{~d}$ and one at $9 \mathrm{~d}$ ), one SM observation (at $-8 \mathrm{~d}$ ), and one PE observation (at $9 \mathrm{~d}$ ) were removed from the AT data. The repeated-measures ANOVA were performed using the PROC MIXED procedure, in which the treatments, times, and their interactions were set as the fixed effects, block and the interaction between block and time were set as random effects, and the animal was set as within-subject effect. Three covariance structures were used in the analyses of variables in the AT dataset, including compound symmetry (CS), unstructured (UN), and spatial power law SP (POW). The structure that gave the smallest Bayesian information criterion (BIC) was eventually selected. In addition, the slice option of MIXED procedure in the SAS software was used to test the effect of diet within each time point. The $P$-values $\leq 0.05$ were considered as statistically significant and $0.05<P<0.10$ was considered as a tendency toward significance.

\section{Results}

3.1 Effect of prepartal overfeeding on lipid subspecies profiles

Only a few detected masses in the datasets exceeded the FDR threshold calculation (Online Resource Table S3-S6). Significant concentration differences between treatments were only found for seven detected masses at -8 d in AT ESIdataset wherein $7 \times 0.11529$ (threshold, adjusted- $P$ ) produced less than 1 false positive (Table 1 ). Five out of the seven significant lipids were Cer subspecies, with one PE and one unknown subspecies being the rest.

\subsection{Contribution of lipids to the distinctness between groups}

The lipidomic profiles of the liver and the AT presented clear distinctness after PCA-LDA. The first 2 PL functions contributed to $99.17 \%$ of the distinctness between all groups in the two liver datasets (Online Resource Fig. S1A and S1B). The first three PL functions contributed to $86.18 \%$ and $90.93 \%$ of the separation between all groups in AT ESI+ dataset and AT ESI- dataset respectively (Online Resource Fig. S2A and S2B). The PL2 of both liver datasets differentiated between the two feeding groups, whereas PL1 differentiated the time points. The lipidomic profiles of the two feeding groups in the AT datasets were categorized according to PL1 in ESI+ and by PL2 in ESI-. The lipidomic profiles on different time points were categorically separated by different PLs in the two feeding groups, which indicated that the lipidomic profiles in the two feeding groups may change following different patterns across the periparturition period.

The plots of corresponding PL functions against the retention time of the lipids in MS provided an overview of the contribution of each lipid class made to the difference by diet effect in the PCA-LDA (Fig. 1). The distinctness between feeding groups in the liver ESI+ dataset resulted from changes in levels of phospholipids, lysophospholipids, Cers, and 
SMs. In liver ESI- dataset, the separation between feeding groups was mainly resulted from phospholipids and Cers. The difference between the feeding groups in the two AT data sets was equally contributed to by the lipids. As the separation between time points was achieved by different PL in the two feeding groups, the analyses of PL loadings against retention time for the time effect were not processed in the AT datasets.

The PL loadings of lipids indicated an abundance of lipids, and in very small concentrations loadings of lipids were not likely to have made a considerable contribution to the distinctness of the overall lipidomic profiles. The top 20 lipids that contributed most to each profile were picked up after the lipids were ranked according to the absolute value of the corresponding PL loadings (Table 2 and 3). The ranking indicated that the separation between feeding groups was mainly caused by PCs, PEs, LysoPCs, and LysoPEs in the liver ESI+ dataset, whereas the separation between time points was mainly caused by PCs, PEs, and TGs. The changes in Cer and PE quantities were the main contributors for the differentiation between both feeding groups and time points in the liver ESI- dataset. The differentiation between feeding groups for the AT ESI+ dataset was mainly contributed to by TGs. In AT ESI- dataset, Cers, PEs, and PIs accounted for the differences between feeding groups.

The significant ANOVA effects of the lipid subspecies of diet revealed a considerable proportion of the lipids to be in the liver $(P<0.05)$ (Online Resource Table S7). In contrast, only a few lipids displayed differences between feeding groups in AT $(P<0.05)$, which suggest a marked similarity of the lipidomic profiles between the two feeding groups in AT. The combination of PCA-LDA and repeated measures ANOVA enabled the selection of the lipids based on both their statistical and biological significance. The lipids that showed significant diet or time effects in the iterative ANOVA and which were ranked in the top 20 lipids in PCA-LDA were identified as the subspecies that explained most of the variation between diets or time points (Online Resource Table S7). Consequently, 12 lipids were identified as having contributed most to the distinctness between the feeding groups in the liver ESI+ dataset, including 1 LysoPE, 1 TG, 2 LysoPCs, 4 PCs, and 4 PEs (Table 2). Six lipids were identified as having contributed most to the separation between feeding groups in the liver ESI- dataset, including 1 Cer, 1 PC, and 4 PEs (Table 2). Fourteen lipids were identified as having contributed most to the separation between time points in liver ESI+ dataset, and these comprised 1 DG, 1 Cer, 1 SM, 3 TGs, 4 PCs, and 4 PEs (Table 2). Fifteen lipids contributed most to the separation between time points in the liver ESI- dataset, included 1 Hex, 1 LysoPC, 3 PCs, 4 PEs, and 6 Cers (Table 2). Significant differences between feeding groups were observed in the AT but only one SM species in the top 20 lipids list of the ESI+ dataset was found and 3 Cer subspecies in the ESI- dataset were identified (Table 3).

The HIGH group displayed higher hepatic LysoPC, LysoPE, PE, PI, and SM concentrations $(P<0.05)$ during the periparturient period than the CON group (Fig. 2). Concentrations of PCs and total lipids tended to be higher in the HIGH group in this period $(P<0.10)$. It is noteworthy that the differences in the lipid concentrations between the two feeding regimes were mainly derived from the prepartum data. The HIGH group at $-8 \mathrm{~d}$ had higher concentrations of LysoPC, PC, PI, SM, and total lipids ( $P<0.05)$ and tended to have higher PE concentrations compared to the CON group (Fig. 2). However, some of these differences diminished after parturition, and were characterized by only higher LysoPC and PE concentrations and a tendency towards higher SM concentration in the HIGH group compared to the CON group. The 
concentrations of PI, DG, TG, and total lipids increased for both groups after parturition $(P<0.05)$, whereas SM had a tendency to decrease across the time points $(P=0.10)$ (Fig. 2).

Prepartal overfeeding only increased the concentration of Cer in the AT of the HIGH group during the whole periparturient period $(P<0.05)$ (Fig. 3). Prepartal overfeeding at specific time points resulted in higher Cer and PI concentrations and tended to increase PC and PE at $-8 \mathrm{~d}$. Increased levels of Cer and decreased levels of SM were found in the HIGH group at $1 \mathrm{~d}$. However, all the differences between groups had diminished by $9 \mathrm{~d}$ (Fig. 3). The concentrations of most lipid classes displayed no alteration when comparing both feeding groups during the periparturient period except that the concentrations of SM increased across time $(P<0.05)$. In addition, significant interactions or tendencies of interaction between diet and time were found for SM $(P<0.05)$, PC $(P<0.05)$, PE $(P<0.10)$ and PI $(P<0.05)$ (Fig. 3). These interactions were presented as a delayed increase in SM concentrations in the HIGH group compared to the CON group, and decreases in the change of PC, PE, and PI levels between the two feeding groups (Fig. 3).

\section{Discussion}

The effects of prepartal overfeeding on the lipidomic profiles of dairy cows on lipid subspecies and lipid classes were analyzed by using three different approaches, including the ANOVA between groups within time points on all lipid subspecies, PCA-LDA, and the repeated measures ANOVA of the total concentrations of lipid classes. The strict FDR threshold calculation in the screening of individual lipids enabled the identification of the subspecies that were most affected by the prepartal overfeeding. Only a very small proportion of lipids finally passed the threshold, i.e., seven lipids in AT at -8 d. These lipids can be interpreted as the most sensitive subspecies to the prepartal overfeeding and may have roles in the development of IR. The majority of the lipids that exceeded the FDR threshold were ceramides, which contain long-chain fatty acids of at least 20 carbons. The significant change of Cer profile caused by prepartal overfeeding was also confirmed by the other analytical approaches because Cer lipids were found to be affected by the diet by the PCALDA of the AT dataset and there was also a significant difference in Cer profiles in AT between the two feeding groups determined by the class-based analysis. Moreover, the class-based analysis showed that the difference in Cer concentrations between the two feeding groups in AT diminished after parturition, which could be attributed to the termination of overfeeding in the HIGH group at parturition. The elevation in Cer (16:0), Cer (24:0) and total Cer concentrations were previously reported in the plasma of overweight dairy cows compared to lean dairy cows (Rico et al. 2015). It has been suggested that liver is the major source of plasma Cer (Lightle et al. 2003; Yamaguchi et al. 2004). Although the class-based analysis of the liver displayed no significant difference in the hepatic Cer concentrations between $\mathrm{CON}$ and $\mathrm{HIGH}$ groups during the periparturient period, a number of Cer subspecies did contribute to the difference between the two feeding groups in liver ESI- dataset as determined by PCA-LDA. The reason for the different outcomes between the two analyses for the liver data could be that only Cers containing specific fatty acids were significantly altered by the prepartal overfeeding.

Ceramides have been studied as potential triggers for IR in the human and the mouse, thus they have been identified as a biomarker for diabetes in the human (Lappas et al. 2015). The accumulation of ceramides were suggested to reduce the insulin-stimulated glucose transport in adipocyte cultures (Summers et al. 1998). The onset of insulin resistance in mice was found to be accompanied by increased local Cer concentration in the AT, when obesity was induced by a high-fat diet (Turner et al. 2013). On the other hand, the depletion of Cer de novo synthesis by a chemical inhibitor or the knockout 
of synthetic enzymes effectively improved the insulin sensitivity in mice (Holland et al. 2007; Yang et al. 2009). Further, in vitro and in vivo studies on the molecular level have demonstrated that Cers prevented the translocation of protein kinase $\mathrm{B}(\mathrm{Akt} / \mathrm{PKB})$ in phosphoinositide 3 kinase (PI3-K) of the insulin signaling pathway through the activation of either protein kinase C (PKC) or protein phosphatase 2 A (PP2A) in the muscle (Chavez et al. 2003; Fox et al. 2007). Based on the intravenous glucose tolerance test (IVGTT) performed on the following day of biopsies from the same cows in the present study, HIGH group showed a blunted suppression of plasma NEFA compared to CON group at $-8 \mathrm{~d}$, suggesting increased IR in AT caused by prepartal overfeeding at $-8 \mathrm{~d}$ (Kokkonen et al. 2012). Therefore, the higher Cer concentrations in HIGH group corresponded to the results from IVGTT and may reflect greater magnitude of IR in AT of the HIGH group before parturition.

The concentration patterns of Cer in the AT found in the present study were similar for both feeding groups, which was characterized by an increase from $-8 \mathrm{~d}$ to $1 \mathrm{~d}$ followed by a decrease to a lower level compared to that at $-8 \mathrm{~d}$. Dairy cows have increased IR during late pregnancy and early lactation (Bell and Bauman 1997). The decline in Cer concentrations in the AT may suggest decreased IR after the parturition. The change of Cers over the entire periparturient period reflected a potential transition in cellular processes, with parturition being a trigger for that transition.

As the most abundant complex sphingolipids in mammalian cells, SMs are synthesized by the transfer of a phosphocholine headgroup from PC to Cer, which yields another product DG at the same time (Gault et al. 2010). Conversely, SM can be hydrolyzed to Cers when the reaction is mediated by acid sphingomyelinase (Kitatani et al. 2008). Therefore, SM and Cer lipids are closely associated with each other through the biological conversion. The conversion between SM and Cer is regulated by various cellular stimuli. The evidence from in vitro studies suggest that the hydrolysis of SM to Cer was potentially promoted by oxidative stress (Gault et al. 2010), adipokine tumor necrosis factor alpha (TNF $\alpha$ ) (Dbaibo et al. 2001), and by the inflammatory response that is induced by Toll-like receptor 4 (Holland et al. 2011). The concentrations of SM lipids in the liver were found to be higher in the HIGH group compared to CON group during the periparturient period, which suggest reduced SM hydrolyses. Previously, increased concentrations of SM subspecies were found in the serum of obese humans with lower insulin sensitivity (Hanamatsu et al. 2014). However, contrasting results were reported showing decreased SM concentrations in the liver of obese mice with hepatic steatosis (Yetukuri et al. 2007). It is noteworthy that the HIGH group did not have a decrease of hepatic Cer concentrations in response to the increase of SM species. Therefore, Cer lipids in the liver may be produced through multiple pathways, including de novo synthesis pathway and salvage pathways (Merrill 2011). The interaction between diet and time of SM in AT can be interpreted as a delay of increase in the HIGH group. Abuelo et al. (2015) demonstrated that oxidative stress is increased in dairy cows after calving, which potentially promotes the production of Cer species from SM. However, the concentrations of Cer lipids, decreased in both groups after parturition, which was in contrast to the change of SM concentrations across the time. This conflicting result suggests that production of Cers can be mediated by other pathways than SM hydrolysis or was dominantly mediated by other factors than oxidative stress.

Only a few LysoPC and LysoPE subspecies were identified in the liver and in the AT. However, the PCA-LDA results showed a small contribution of lysophospholipids to the difference in the hepatic lipidomic profiles between the two feeding groups. Although small, the total concentrations of LysoPC and LysoPE in the liver were significantly higher for the HIGH group from $-8 \mathrm{~d}$ to $9 \mathrm{~d}$. Previously, the lipidomic profiling of dairy cow plasma indicated that the concentration of 5 LysoPC subspecies, required for very-low-density lipoprotein (VLDL) secretion, decreased from two weeks 
prepartum until parturition and then increased thereafter until four weeks postpartum (Imhasly et al. 2015). Their results indicate the potential roles of LysoPC in metabolic adaptation of dairy cows during the periparturient period. Therefore, the difference in lysophospholipid concentrations between CON and HIGH groups may indicate differences in the physiological adaptation during the periparturient period. Specific LysoPC subspecies in humans and mice were found to be associated with insulin resistance. Pietilainen et al. (2007) demonstrated that LysoPC concentration was higher in the serum of the obese twin compared to that in the serum of normal-weight twin. Kim et al. (2011) also reported that LysoPC concentration was higher in the plasma of overweight humans compared to that of their normal-weight counterparts. However, the opposite result was reported by Wallace et al. (2014): a finding that suggested humans with higher body mass indices (BMI) had lower plasma concentrations of LysoPC and LysoPE subspecies. Moreover, Wallace and colleagues (2014) also reported that plasma concentrations of these lipids were negatively correlated with homeostasis model assessment-estimated insulin resistance (HOMA-IR).

The phospholipids identified in the liver and in AT included PC, PE, and PI subspecies. These phospholipids are required for the secretion of hepatic TG as VLDL particles in the liver (Vernon 2005). They are also essential components of lipid droplets and thus their abundance may be related to the accumulation of TG. In addition, specific phospholipid subspecies were found to act as cell signaling messengers that regulate the action of PPAR $\alpha$ (Chakravarthy et al. 2009). The concentrations of PC species in dairy cows decreased from two weeks prepartum until parturition and then increased until four weeks after calving (Imhasly et al. 2015). The same group reported in an earlier study that six PC subspecies may have significant roles in the variation of the plasma lipidomic profiles between healthy dairy cows and cows with a different degree of fat deposition in the hepatocytes (Imhasly et al. 2014). They stated the decrease of PC (36:3), PC (38:3), PC (36:4) and PC (36:2) and the increase of PC (30:2) and PC (32:2) in the plasma of the cows with hepatic lipidosis in comparison to the healthy cows. Among these PC subspecies, increased concentration of PC (32:2) in the HIGH group was observed in liver ESI+ dataset in the present study.

Much more profiling on phospholipids has been conducted in human and mice suffering from obesity and associated metabolic diseases. Eisinger and colleagues analyzed the lipidomic profiles in the liver of mice and found lower concentrations of total PC, saturated fatty acids (SFA) PC, monounsaturated fatty acids (MUFA) PE, and various PI subspecies in high-fat fed mice compared with control mice (Eisinger et al. 2014b). The PC and PE subspecies also decreased in animals with steatohepatitis when compared with healthy animals (Koteish and Diehl 2001). Specific PC and PE subspecies in humans were found to be potential predictor of type 2 diabetes in women with previous gestational diabetes mellitus (Lappas et al. 2015). The PCA-LDA revealed that phospholipids had significant contributions to the difference in hepatic and AT lipidomic profiles between the CON group and the HIGH group in our study. The increase or the tendency to increase in PC, PE, and PI concentrations in the liver caused by prepartal overfeeding were also identified in the class-based analyses. The rise of phospholipids in the liver of the HIGH group may suggest increased VLDL secretion from the liver during the periparturient period (Oikawa et al. 2010). Alternatively, it may indicate increased lipid deposition in the liver caused by the oversupply of energy based on the tendency for higher total lipid concentrations in the liver (Fig. 2). The phospholipid concentrations in the AT tended to be higher for the HIGH group on $-8 \mathrm{~d}$ compared to that of the CON group. The significant interaction between diet and time in the class-based analyses indicated that the concentration of phospholipids changed and exhibited opposite patterns in the two groups. This interaction was supported by the results from PCA-LDA, as no single PL function was able to explain the separation of 
lipidomic profiles by time in the both feeding groups. The different changing patterns of phospholipids across the time reflected the difference in the physiological adaptation in the two feeding groups.

359 The neutral lipids are the main components of the lipid deposition in mammals. The accumulation of TG has been observed in the liver of dairy cows with hepatic lipidosis in numerous earlier studies (Lubojacka et al. 2005; Murondoti et al. 2004). The concentration of TG in the liver in our study increased dramatically across the time points, which indicates that hepatic lipid accumulation after parturition was driven by the greater lipolysis in AT caused by the negative energy balance. As the DG is the intermediate in TG synthesis, the DG levels increased following a similar pattern after parturition. Moreover, the increase of lipid deposition in the liver was also reflected by the significant elevation of total lipid concentration after parturition. Moreover, the tendency towards higher total lipid concentration in HIGH group during the periparturient period may reflect higher risk to develop fatty liver in HIGH group compared to CON group.

Collectively, the lipidomic profiles revealed changes in various lipid classes during the periparturient period. The global lipidomic profiling in the present study was conducted using a conventional extraction method fitting to wide variety of lipid classes. However, specific lipids, including glycolipids and acidic phospholipids, may require further optimization of the extraction solvent and rations of solvents in the extraction.. Therefore, the identification of these lipid classes may have been restricted in the present study. Moreover, the incompleteness of the internal library limited the identification of lipids. However, the global profiling of the whole lipidome still provided novel insights of the influence of prepartal overfeeding on the lipid metabolism in periparturient dairy cows.

\section{Conclusions}

The dairy cows that received a high-energy diet from six weeks prepartum until parturition displayed altered lipidomic profiles in their livers and in their adipose tissue compared to the cows that received a controlled-energy diet. Prepartal overfeeding increased the concentrations of various phospholipids, lysophospholipids, and sphingomyelins in the liver during the periparturient period. The adipose tissue of the overfed cows had the higher concentrations of ceramides and displayed an opposite change of phospholipid profile after the parturition compared to the cows that received the controlled-energy diet. The increase of sphingomyelins in the liver and the increase of ceramides in the adipose tissue of the overfed cows potentially reflected the different magnitude of IR in the overfed cows compared to the cows in the controlled-energy diet. Therefore, the results of lipidomic profiling in this study provided a novel perspective on the molecular mechanisms underlying the insulin resistance in dairy cows during the periparturient period. Future studies should investigate the biosynthesis pathways of the lipids that were significantly influenced by prepartal energy level to understand further the mechanism of IR in dairy cows.

Compliance with ethical standards The experimental procedures were conducted under the protocols approved by the National Animal Ethics Committee in Finland.

Conflict of interest The authors declare that they have no conflict of interest. 


\section{References}

Abuelo, A., Hernandez, J., Benedito, J.L. and Castillo, C. (2015). The importance of the oxidative status of dairy cattle in the periparturient period: Revisiting antioxidant supplementation. Journal of Animal Physiology and Animal Nutrition, 99, 1003-1016.

Bell, A.W. and Bauman, D.E. (1997). Adaptations of glucose metabolism during pregnancy and lactation. Journal of Mammary Gland Biology and Neoplasia, 2, 265-278.

Benjamini, Y. and Hochberg, Y. (2000). On the adaptive control of the false discovery fate in multiple testing with independent statistics. Journal of Educational and Behavioral Statistics, 25, 60-83.

Bertics, S.J., Grummer, R.R., Cadornigavalino, C. and Stoddard, E.E. (1992). Effect of prepartum dry-matter intake on liver triglyceride concentration and early lactation. Journal of Dairy Science, 75, 1914-1922.

Chakravarthy, M.V., Lodhi, I.J., Yin, L., Malapaka, R.R.V., Xu, H.E., Turk, J. et al. (2009). Identification of a physiologically relevant endogenous ligand for PPAR alpha in liver. Cell, 138, 476-488.

Chavez, J.A., Knotts, T.A., Wang, L.P., Li, G.B., Dobrowsky, R.T., Florant, G.L. et al. (2003). A role for ceramide, but not diacylglycerol, in the antagonism of insulin signal transduction by saturated fatty acids. Journal of Biological Chemistry, 278, 10297-10303.

Chavez, J.A. and Summers, S.A. (2010). Lipid oversupply, selective insulin resistance, and lipotoxicity: Molecular mechanisms. Biochimica et Biophysica Acta-Molecular and Cell Biology of Lipids, 1801, 252-265.

Coppock, C.E. (1985). Energy nutrition and metabolism of the lactating dairy-cow. Journal of Dairy Science, 68, 34033410 .

Dbaibo, G.S., El-Assaad, W., Krikorian, A., Liu, B., Diab, K., Idriss, N.Z. et al. (2001). Ceramide generation by two distinct pathways in tumor necrosis factor alpha-induced cell death. FEBS Letters, 503, 7-12.

De Koster, J.D. and Opsomer, G. (2013). Insulin resistance in dairy cows. Veterinary Clinics of North America-Food Animal Practice, 29, 299-322.

Douglas, G.N., Overton, T.R., Bateman, H.G., Dann, H.M. and Drackley, J.K. (2006). Prepartal plane of nutrition, regardless of dietary energy source, affects periparturient metabolism and dry matter intake in holstein cows. Journal of Dairy Science, 89, 2141-2157.

Drackley, J.K., Richard, M.J., Beitz, D.C. and Young, J.W. (1992). Metabolic changes in dairy-cows with ketonemia in response to feed restriction and dietary 1,3-butanediol. Journal of Dairy Science, 75, 1622-1634.

Drackley, J., Dann, H., Douglas, G., Guretzky, N., Litherland, N., Underwood, J. et al. (2005). Physiological and pathological adaptations in dairy cows that may increase susceptibility to periparturient diseases and disorders. Italian Journal of Animal Science, 4, 323-344.

Eisinger, K., Krautbauer, S., Hebel, T., Schmitz, G., Aslanidis, C., Liebisch, G. et al. (2014a). Lipidomic analysis of the liver from high-fat diet induced obese mice identifies changes in multiple lipid classes. Experimental and Molecular Pathology, 97, 37-43.

Eisinger, K., Liebisch, G., Schmitz, G., Aslanidis, C., Krautbauer, S. and Buechler, C. (2014b). Lipidomic analysis of serum from high fat diet induced obese mice. International Journal of Molecular Sciences, 15, 2991-3002.

Erion, D.M. and Shulman, G.I. (2010). Diacylglycerol-mediated insulin resistance. Nature Medicine, 16, 400-402. 
Fearn, T. (2008). Principal component discriminant analysis. Statistical Applications in Genetics and Molecular Biology, 7, 6 .

Fox, T.E., Houck, K.L., O'Neill, S.M., Nagarajan, M., Stover, T.C., Pomianowski, P.T. et al. (2007). Ceramide recruits and activates protein kinase C zeta (PKC zeta) within structured membrane microdomains. Journal of Biological Chemistry, 282, 12450-12457.

Gault, C.R., Obeid, L.M. and Hannun, Y.A. (2010). An overview of sphingolipid metabolism: From synthesis to breakdown. Sphingolipids as Signaling and Regulatory Molecules, 688, 1-23.

Hanamatsu, H., Ohnishi, S., Sakai, S., Yuyama, K., Mitsutake, S., Takeda, H. et al. (2014). Altered levels of serum sphingomyelin and ceramide containing distinct acyl chains in young obese adults. Nutrition \& Diabetes, 4 , e141.

Haus, J.M., Kashyap, S.R., Kasumov, T., Zhang, R., Kelly, K.R., DeFronzo, R.A. et al. (2009). Plasma ceramides are elevated in obese subjects with type 2 diabetes and correlate with the severity of insulin resistance. Diabetes, 58, 337343.

Hayirli, A. (2006). The role of exogenous insulin in the complex of hepatic lipidosis and ketosis associated with insulin resistance phenomenon in postpartum dairy cattle. Veterinary Research Communications, 30, 749-774.

Holland, W.L., Bikman, B.T., Wang, L., Yuguang, G., Sargent, K.M., Bulchand, S. et al. (2011). Lipid-induced insulin resistance mediated by the proinflammatory receptor TLR4 requires saturated fatty acid-induced ceramide biosynthesis in mice. Journal of Clinical Investigation, 121, 1858-1870.

Holland, W.L., Brozinick, J.T., Wang, L., Hawkins, E.D., Sargent, K.M., Liu, Y. et al. (2007). Inhibition of ceramide synthesis ameliorates glucocorticoid-, saturated-fat-, and obesity-induced insulin resistance. Cell Metabolism, 5, 167179.

Holtenius, K., Agenas, S., Delavaud, C. and Chilliard, Y. (2003). Effects of feeding intensity during the dry period. 2. metabolic and hormonal responses. Journal of Dairy Science, 86, 883-891.

Imhasly, S., Bieli, C., Naegeli, H., Nystroem, L., Ruetten, M. and Gerspach, C. (2015). Blood plasma lipidome profile of dairy cows during the transition period. BMC Veterinary Research, 11, 252.

Imhasly, S., Naegeli, H., Baumann, S., von Bergen, M., Luch, A., Jungnickel, H. et al. (2014). Metabolomic biomarkers correlating with hepatic lipidosis in dairy cows. BMC Veterinary Research, 10, 122.

Ingvartsen, K.L. (2006). Feeding- and management-related diseases in the transition cow - physiological adaptations around calving and strategies to reduce feeding-related diseases. Animal Feed Science and Technology, 126, 175-213.

Ji, P., Osorio, J.S., Drackley, J.K. and Loor, J.J. (2012). Overfeeding a moderate energy diet prepartum does not impair bovine subcutaneous adipose tissue insulin signal transduction and induces marked changes in peripartal gene network expression. Journal of Dairy Science, 95, 4333-4351.

Kahn, B.B. and Flier, J.S. (2000). Obesity and insulin resistance. Journal of Clinical Investigation, 106, 473-481.

Khan, M.J., Jacometo, C.B., Graugnard, D.E., Correa, M.N., Schmitt, E., Cardoso, F. et al. (2014). Overfeeding dairy cattle during late-pregnancy alters hepatic PPARalpha-regulated pathways including hepatokines: Impact on metabolism and peripheral insulin sensitivity. Gene Regulation and Systems Biology, 8, 97-111.

Kim, H., Kim, J.H., Noh, S., Hur, H.J., Sung, M.J., Hwang, J. et al. (2011). Metabolomic analysis of livers and serum from high-fat diet induced obese mice. Journal of Proteome Research, 10, 722-731.

Kitatani, K., Idkowiak-Baldys, J. and Hannun, Y.A. (2008). The sphingolipid salvage pathway in ceramide metabolism and signaling. Cellular Signalling, 20, 1010-1018. 
Kokkonen, T., Salin, S., Selim, S., Taponen, J., Elo, K. \& Vanhatalo, A.. 2012. Effect of dietary energy level during the dry period on insulin resistance in dairy cows. In: Proceedings of the 3rd Nordic Feed Science Conference, 28. -29.6. 2012, Uppsala (ed. P.Uden et al.). Available at http://www.slu.se/en/departments/animal-nutrition-

471 management/publications/, pp. 84-88.

Koteish, A. and Diehl, A.M. (2001). Animal models of steatosis. Seminars in Liver Disease, 21, 89-104.

Lappas, M., Mundra, P.A., Wong, G., Huynh, K., Jinks, D., Georgiou, H.M. et al. (2015). The prediction of type 2 diabetes in women with previous gestational diabetes mellitus using lipidomics. Diabetologia, 58, 1436-1442.

Lightle, S., Tosheva, R., Lee, A., Queen-Baker, J., Boyanovsky, B., Shedlofsky, S. et al. (2003). Elevation of ceramide in serum lipoproteins during acute phase response in humans and mice: Role of serine-palmitoyl transferase. Archives of Biochemistry and Biophysics, 419, 120-128.

Loor, J.J., Dann, H.M., Guretzky, N.A.J., Everts, R.E., Oliveira, R., Green, C.A. et al. (2006). Plane of nutrition prepartum alters hepatic gene expression and function in dairy cows as assessed by longitudinal transcript and metabolic profiling. Physiological Genomics, 27, 29-41.

Lubojacka, V., Pechova, A., Dvorak, R., Drastich, P., Kummer, V. and Poul, J. (2005). Liver steatosis following supplementation with fat in dairy cow diets. Acta Veterinaria Brno, 74, 217-224.

McArdle, M.A., Finucane, O.M., Connaughton, R.M., McMorrow, A.M. and Roche, H.M. (2013). Mechanisms of obesity-induced inflammation and insulin resistance: Insights into the emerging role of nutritional strategies. Frontiers in Endocrinology, 4, 52-52.Merrill, A.H.,Jr. (2011). Sphingolipid and glycosphingolipid metabolic pathways in the era of sphingolipidomics. Chemical Reviews, 111, 6387-6422.

Murondoti, A., Jorritsma, R., Beynen, A.C., Wensing, T. and Geelen, M.J.H. (2004). Activities of the enzymes of hepatic gluconeogenesis in periparturient dairy cows with induced fatty liver. Journal of Dairy Research, 71, 129-134.

Nielsen, N.I., Hameleers, A., Young, F.J., Larsen, T. and Friggens, N.C. (2010). Energy intake in late gestation affects blood metabolites in early lactation independently of milk production in dairy cows. Animal, 4, 52-60.

Nygren, H., Seppanen-Laakso, T., Castillo, S., Hyotylainen, T. and Oresic, M. (2011). Liquid chromatography-mass spectrometry (LC-MS)-based lipidomics for studies of body fluids and tissues. Metabolic Profiling: Methods and Protocols, 708, 247-257.

Oikawa, S., Mizunuma, Y., Iwasaki, Y. and Tharwat, M. (2010). Changes of very low-density lipoprotein concentration in hepatic blood from cows with fasting-induced hepatic lipidosis. Canadian Journal of Veterinary Research-Revue Canadienne De Recherche Veterinaire, 74, 317-320.

Pietilainen, K.H., Sysi-Aho, M., Rissanen, A., Seppanen-Laakso, T., Yki-Jarvinen, H., Kaprio, J. et al. (2007). Acquired obesity is associated with changes in the serum lipidomic profile independent of genetic effects - A monozygotic twin study. Plos One, 2, e218.

Rauschert, S., Uhl, O., Koletzko, B., Kirchberg, F., Mori, T.A., Huang, R. et al. (2016). Lipidomics reveals associations of phospholipids with obesity and insulin resistance in young adults. The Journal of Clinical Endocrinology and Metabolism, 101, 871-9.

Reid, I.M. (1980). Incidence and severity of fatty liver in dairy-cows. Veterinary Record, 107, 281-284.

Rico, J.E., Bandaru, V.V.R., Dorskind, J.M., Haughey, N.J. and McFadden, J.W. (2015). Plasma ceramides are elevated in overweight holstein dairy cows experiencing greater lipolysis and insulin resistance during the transition from late pregnancy to early lactation. Journal of Dairy Science, 98, 7757-7770.

Rukkwamsuk, T., Wensing, T. and Geelen, M.J.H. (1998). Effect of overfeeding during the dry period on regulation of adipose tissue metabolism in dairy cows during the periparturient period. Journal of Dairy Science, 81, $2904-2911$. 
Selim, S., Kokkonen, T., Taponen, J., Vanhatalo, A. and Elo, K. (2015). Effect of prepartal ad libitum feeding of grass silage on transcriptional adaptations of the liver and subcutaneous adipose tissue in dairy cows during the periparturient period. Journal of Dairy Science, 98, 5515-5528.

Summers, S.A., Garza, L.A., Zhou, H.L. and Birnbaum, M.J. (1998). Regulation of insulin-stimulated glucose transporter GLUT4 translocation and akt kinase activity by ceramide. Molecular and Cellular Biology, 18, 5457-5464.

Turner, N., Kowalski, G.M., Leslie, S.J., Risis, S., Yang, C., Lee-Young, R.S. et al. (2013). Distinct patterns of tissuespecific lipid accumulation during the induction of insulin resistance in mice by high-fat feeding. Diabetologia, 56, 1638-1648.

Veenhuizen, J.J., Drackley, J.K., Richard, M.J., Sanderson, T.P., Miller, L.D. and Young, J.W. (1991). Metabolic changes in blood and liver during development and early treatment of experimental fatty liver and ketosis in cows. Journal of Dairy Science, 74, 4238-4253.

Vernon, R.G. (2005). Lipid metabolism during lactation: A review of adipose tissue-liver interactions and the development of fatty liver. Journal of Dairy Research, 72, 460-469.

Wallace, M., Morris, C., O'Grada, C.M., Ryan, M., Dillon, E.T., Coleman, E. et al. (2014). Relationship between the lipidome, inflammatory markers and insulin resistance. Molecular Biosystems, 10, 1586-1595.

Yamaguchi, M., Miyashita, Y., Kumagai, Y. and Kojo, S. (2004). Change in liver and plasma ceramides during Dgalactosamine-induced acute hepatic injury by LC-MS/MS. Bioorganic \& Medicinal Chemistry Letters, 14, 4061-4064.

Yang, G., Badeanlou, L., Bielawski, J., Roberts, A.J., Hannun, Y.A. and Samad, F. (2009). Central role of ceramide biosynthesis in body weight regulation, energy metabolism, and the metabolic syndrome. American Journal of Physiology-Endocrinology and Metabolism, 297, E211-E224.

Yetukuri, L., Katajamaa, M., Medina-Gomez, G., Seppanen-Laakso, T., Vidal-Puig, A. and Oresic, M. (2007). Bioinformatics strategies for lipidomics analysis: Characterization of obesity related hepatic steatosis. BMC Systems Biology, 1, 12. 
Fig. 1 Plots of PL function against retention time of identified lipid. (a) The second PL function (PL2) indicates the contribution of the variables to the diet effect in the liver positive electrospray ionization mode (ESI+) dataset. (b) The second PL function (PL2) indicates the contributions of the variables to the diet effect in the liver negative electrospray ionization mode (ESI-) dataset. (c) The first PL function (PL1) indicates the contribution of the variables to the diet effect in AT ESI+ dataset. (d) The second PL function (PL2) indicates the contribution of the variables to the diet effect in AT ESI- dataset. (e) The first PL function (PL1) indicated the contribution of the variables to the time effect in liver ESI+ dataset. (f) The first PL function (PL1) indicates the contribution of the variables to the time effect in the liver ESI- dataset. Abbreviations: Cer, ceramide. HexCer, hexosylceramide. SM, sphingomyelin. TG, triacylglycerol. DG, diacylglycerol, ChoE, cholesteryl ester, LysoPC, lysophophosphatidylcholine. LysoPE, lysophosphatidylethanolamine. PC, phosphatidylcholine. PE, phosphatidylethanolamine. PI, phosphatidylinositol.

Fig. 2 Concentrations $(\mu \mathrm{mol} / \mathrm{L})$ of different lipid classes in the liver. The error bars represent the standard deviations calculated from non-transformed concentrations. Abbreviations: Cer, ceramides. HexCer, hexosylceramide. SM, sphingomyelin. TG, triacylglycerol. DG, diacylglycerol. ChoE, cholesteryl ester. LysoPC, lysophophosphatidylcholine. LysoPE, lysophosphatidylethanolamine. PC, phosphatidylcholine. PE, phosphatidylethanolamine. PI, phosphatidylinositol.

Fig. 3 Concentrations $(\mu \mathrm{mol} / \mathrm{L})$ of different lipid classes in the adipose tissue. The error bars represent the standard deviation calculated from non-transformed concentration. Cer, ceramide. HexCer, hexosylceramide. SM, sphingomyelin. TG, triacylglycerol. DG, diacylglycerol, ChoE, cholesteryl ester. LysoPC, lysophophosphatidylcholine. LysoPE, lysophosphatidylethanolamine. PC, phosphatidylcholine. PE, phosphatidylethanolamine. PI, phosphatidylinositol. 
A) Liver ESI+

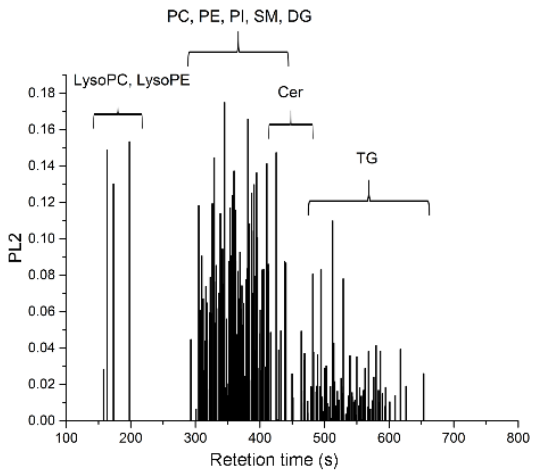

C) AT ESI+

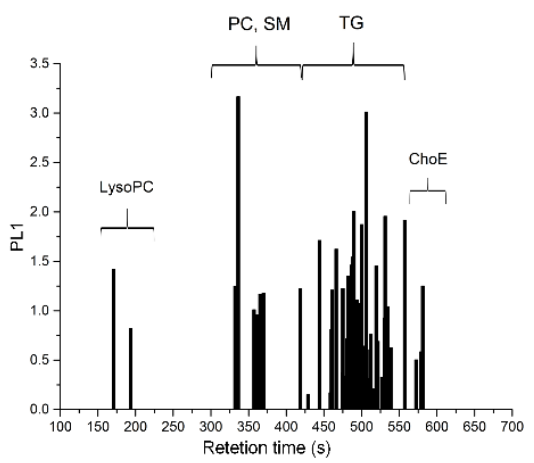

E) Liver ESI+

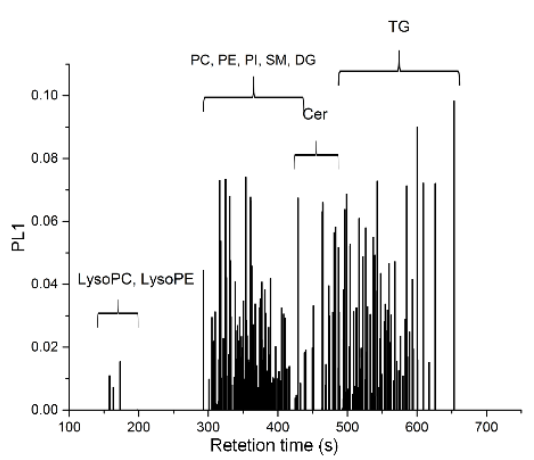

B) Liver ESI-

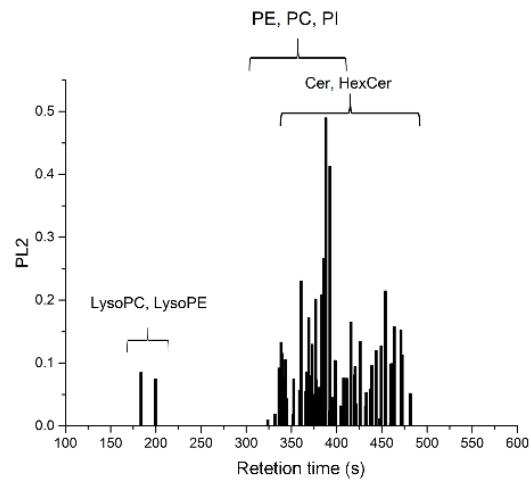

D) AT ESI-

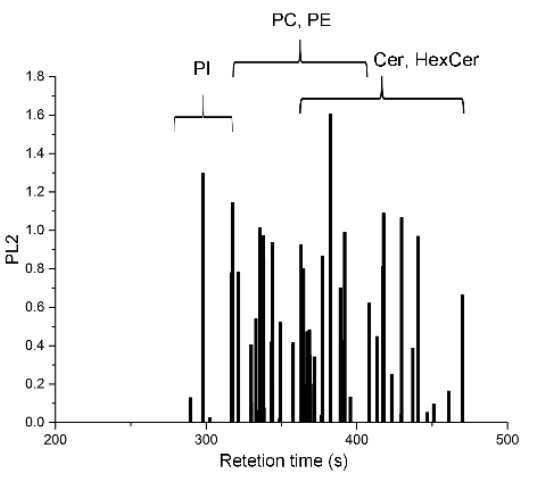

F) Liver ESI-

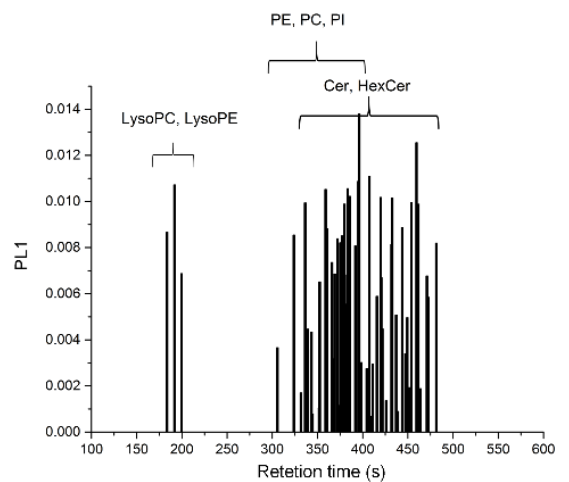



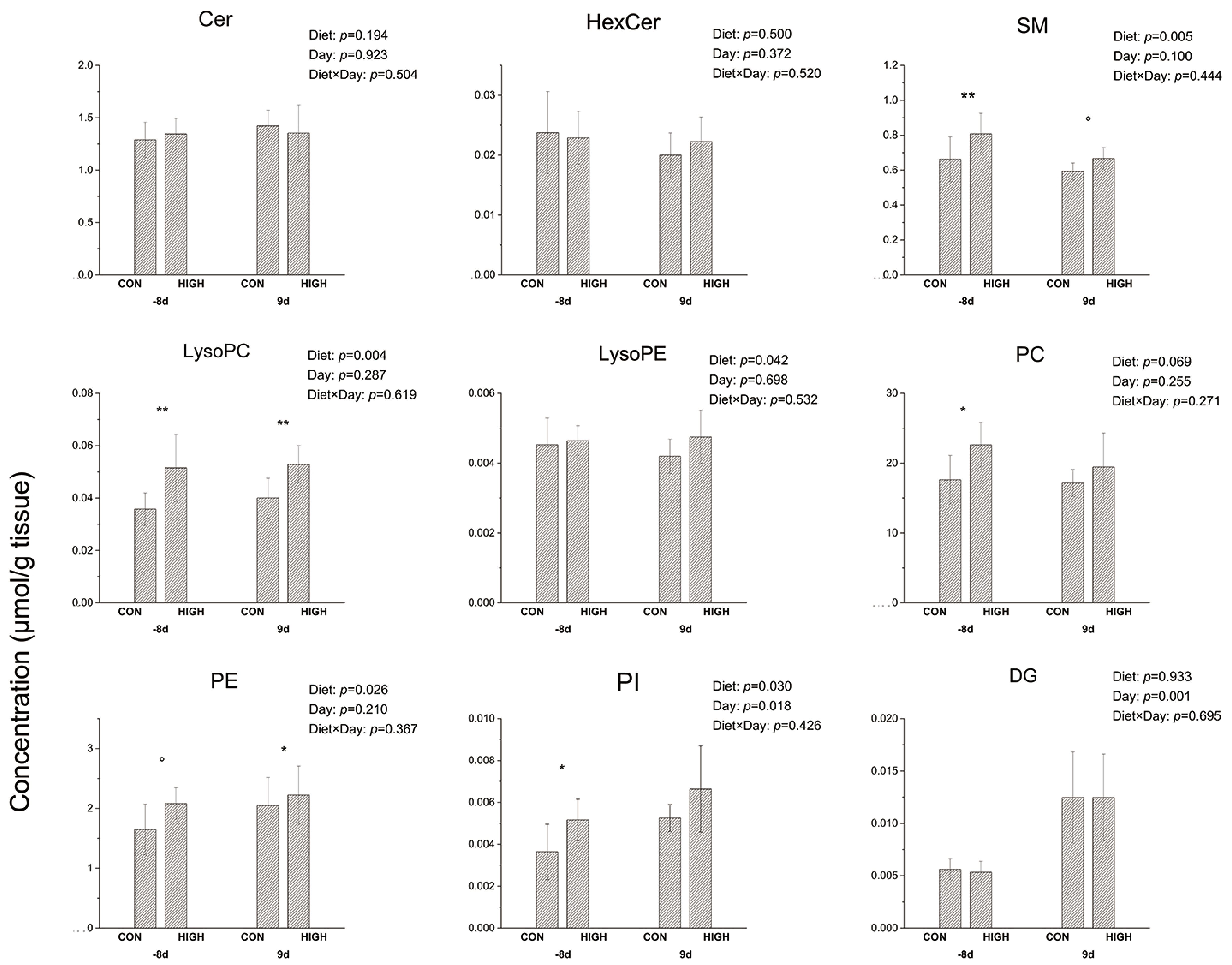

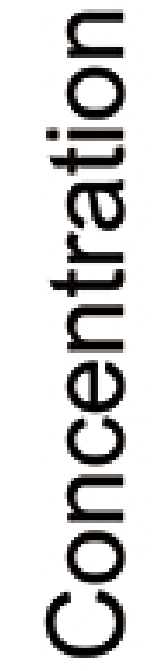
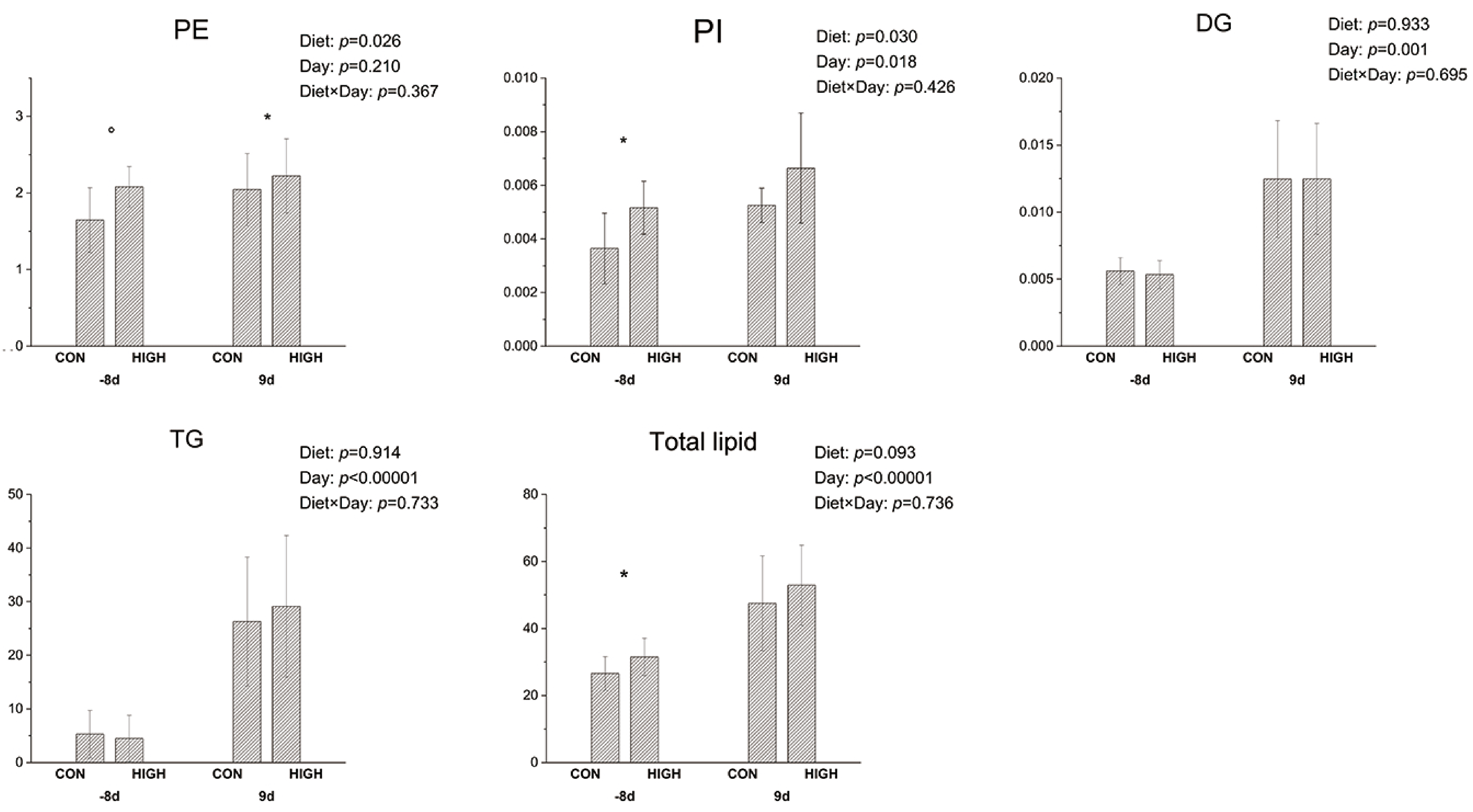

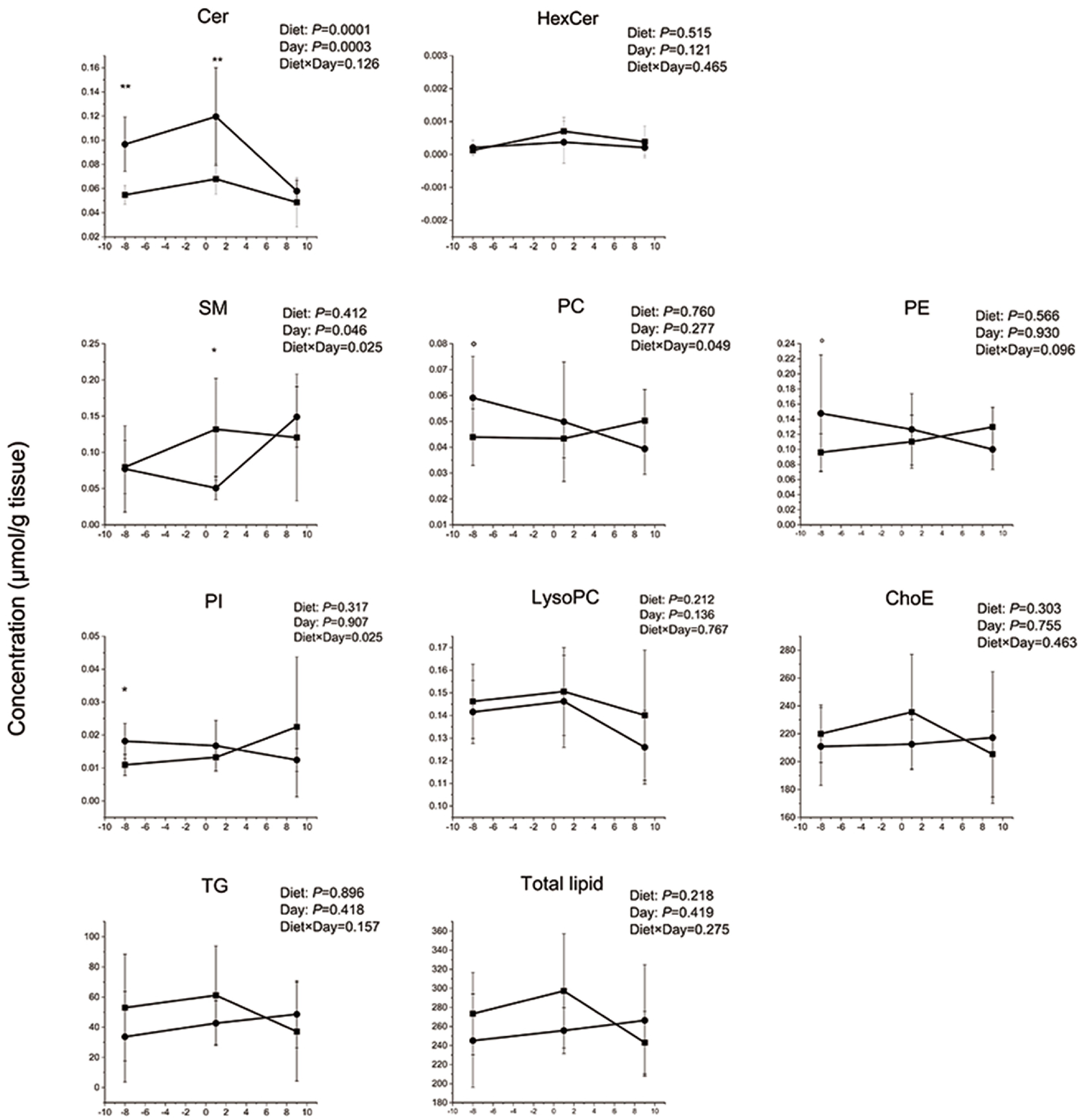

Time relative to parturition (d) 
1 Table 1 Lipids with significantly different concentrations between the two feeding groups. All lipid species were taken 2 from adipose tissue (day -8) and they were measured using negative electrospray ionization mode (ESI-). The values in 3 the table were $\log _{2}$-transformed from the original concentrations $(\mu \mathrm{mol} / \mathrm{L})$.

\begin{tabular}{lllll}
\hline Species & CON $^{\mathrm{a}}$ & HIGH $^{\mathrm{b}}$ & Adjusted- $^{\mathrm{c}}$ & Log2 fold \\
\hline${ }^{\mathrm{d}}$ Cer(d17:1/22:0) & -9.877 & -8.39 & 0.002 & 1.41 \\
Cer(d18:1/20:0) & -10.999 & -9.443 & 0.003 & 1.44 \\
Cer(d18:1/23:0) & -6.585 & -5.617 & 0.047 & 0.96 \\
Cer(d18:1/22:0) & -6.85 & -5.737 & 0.055 & 1.11 \\
${ }^{\mathrm{e} E}(18: 0 / 20: 0)$ & -9.004 & -8.531 & 0.091 & 0.51 \\
unknown & -10.292 & -9.521 & 0.091 & 0.76 \\
Cer(d18:1/23:1) & -8.329 & -7.174 & 0.115 & 1.15 \\
\hline
\end{tabular}

$4 \quad$ a $\overline{\mathrm{CON}}$, controlled-energy feeding group. ${ }^{\mathrm{b}} \mathrm{HIGH}$, high-energy feeding group. ${ }^{\mathrm{c}}$ Adjusted- $P, P$-value after false discovery 5 rate control. ${ }^{\mathrm{d}} \mathrm{Cer}$, ceramide. ${ }^{\mathrm{P} E}$, phosphatidylethanolamine. 
8 Table 2 Top 20 lipids that contributed to the separation between the lipidomic profiles of HIGH and CON groups or the 9 separation between various time points in the liver datasets.

\begin{tabular}{|c|c|c|c|c|c|c|c|c|}
\hline \multirow{2}{*}{ Rank } & \multicolumn{2}{|c|}{ Liver ESI+ Diet (PL2) } & \multicolumn{2}{|c|}{ Liver ESI- Diet (PL2) } & \multicolumn{2}{|c|}{ Liver ESI+ Day (PL1) } & \multicolumn{2}{|c|}{ Liver ESI- Day (PL1) } \\
\hline & Lipid species & $P$-value & Lipid species & $P$-value & Lipid species & $P$-value & Lipid species & $P$-value \\
\hline$\overline{1}$ & $\overline{P E(32: 1)}$ & $\overline{0.0003 \uparrow}$ & $\mathrm{PC}(33: 1)$ & $0.0013 \uparrow$ & PE(p16:0/22:6) & $<0.0001 \downarrow$ & $\mathrm{PC}(33: 1)$ & $0.0181 \downarrow$ \\
\hline 2 & PE(p18:0/20:4) & 0.0672 & PE(18:3/14:1) & $<0.0001 \uparrow$ & TG(48:5) & 0.8149 & LysoPC(18:0) & $0.0442 \uparrow$ \\
\hline 3 & LysoPC(18:0) & $0.0095 \uparrow$ & PE(p16:0/18:1) & $0.0022 \uparrow$ & $\mathrm{PC}(40: 8)$ & 0.1844 & $\mathrm{PE}(18: 3 / 14: 1)$ & 0.2539 \\
\hline 4 & LysoPE(18:0) & $0.0422 \uparrow$ & PE(p16:0/18:2) & $0.0052 \uparrow$ & DG(33:5) & $0.0055 \uparrow$ & $\operatorname{Cer}(d 18: 1 / 23: 0)$ & $0.0175 \uparrow$ \\
\hline 5 & TG(48:5) & $0.0164 \downarrow$ & Cer(d18:1/23:0) & 0.3932 & $\operatorname{PE}(40: 4 e)$ & $0.0002 \uparrow$ & PE(p16:0/18:1) & $0.0017 \uparrow$ \\
\hline 6 & $\operatorname{PE}(34: 2 e)$ & $0.0021 \uparrow$ & $\mathrm{PC}(38: 3)$ & 0.4884 & $\operatorname{PE}(36: 2 e)$ & 0.7043 & $\mathrm{PE}(\mathrm{p} 16: 0 / 18: 2)$ & 0.0711 \\
\hline 7 & PE(36:0) & $0.0271 \uparrow$ & $\mathrm{PC}(36: 2)$ & 0.1808 & PC(38:6) & $0.0313 \uparrow$ & PC(38:3) & $0.0050 \downarrow$ \\
\hline 8 & $\operatorname{PC}(32: 2)$ & $0.0020 \uparrow$ & Cer(d18:2/18:0) & 0.9884 & $\mathrm{PE}(36: 0)$ & 0.6548 & $\operatorname{Cer}(d 18: 2 / 26: 0)$ & $0.0001 \downarrow$ \\
\hline 9 & $\operatorname{PE}(36: 2 e)$ & 0.0878 & $\operatorname{Cer}(d 18: 2 / 24: 1)$ & $0.0182 \uparrow$ & $\mathrm{TG}(54: 0)$ & 0.3865 & $\operatorname{Cer}(d 18: 2 / 22: 0)$ & $0.0164 \downarrow$ \\
\hline 10 & LysoPC(16:0) & $0.0084 \uparrow$ & Cer(d18:1/24:0) & 0.9882 & $\operatorname{PC}(18: 0 / 22: 6)$ & $0.0015 \downarrow$ & $\operatorname{Cer}(d 18: 2 / 20: 0)$ & $0.0019 \downarrow$ \\
\hline 11 & $\operatorname{PE}(38: 3)$ & 0.5425 & Cer(d18:0/24:0) & 0.5643 & $\operatorname{SM}(d 18: 1 / 16: 0)$ & $0.0402 \downarrow$ & HexCer(d18:2/20:0) & $<0.0001 \downarrow$ \\
\hline 12 & $\operatorname{PC}(34: 2)$ & $0.0106 \uparrow$ & $\begin{array}{l}\text { HexCer(d18:1/22 } \\
: 0)\end{array}$ & 0.8815 & TG(45:0) & $<0.0001 \uparrow$ & $\operatorname{PE}(36: 3)$ & 0.0837 \\
\hline 13 & $\operatorname{PE}(40: 6)$ & 0.6080 & $\begin{array}{l}\text { PE(16:0/20:4)+ } \\
\text { PE(18:2/18:2) }\end{array}$ & $0.0069 \uparrow$ & TG(51:5) & $0.0015 \uparrow$ & Cer(d18:1/25:0) & 0.0883 \\
\hline 14 & $\mathrm{PC}(37: 5)$ & 0.0672 & $\mathrm{PE}(32: 0)$ & 0.6779 & $\mathrm{PC}(34: 3)$ & $0.0223 \uparrow$ & $\operatorname{Cer}(d 18: 2 / 26: 1)$ & $0.0001 \downarrow$ \\
\hline 15 & $\operatorname{SM}(\mathrm{d} 18: 1 / 14: 0)$ & 0.1105 & $\operatorname{Cer}(d 18: 1 / 26: 0)$ & 0.2129 & Cer(d18:2/23:0) & $0.0232 \downarrow$ & PE(36:2) & $\mathbf{0 . 0 0 7 7} \uparrow$ \\
\hline 16 & $\operatorname{PC}(32: 2 e)$ & $0.0126 \uparrow$ & $\operatorname{Cer}(d 18: 1 / 22: 0)$ & 0.2392 & TG(47:3) & $<0.0001 \uparrow$ & PE(18:0/20:3) & $0.0247 \downarrow$ \\
\hline 17 & $\operatorname{PE}(36: 5 e)$ & 0.2484 & $\mathrm{SM}(\mathrm{d} 18: 1 / 16: 0)$ & 0.9610 & $\operatorname{PE}(38: 2)$ & 0.0681 & $\operatorname{PE}(32: 0)$ & 0.1620 \\
\hline 18 & $\mathrm{PC}(38: 4)$ & 0.3370 & $\operatorname{Cer}(d 18: 1 / 25: 0)$ & 0.4881 & PE(38:4) & $0.0034 \downarrow$ & $\mathrm{PC}(16: 0 / 20: 4)$ & $0.0054 \downarrow$ \\
\hline 19 & $\operatorname{PE}(34: 4 e)$ & $0.0026 \uparrow$ & $\begin{array}{l}\mathrm{PC}(16: 0 / 16: 1)+ \\
\mathrm{PC}(14: 0 / 18: 1)^{5}\end{array}$ & 0.1994 & $\operatorname{PC}(38: 3)$ & $0.0032 \downarrow$ & PE(38:4) & $0.0066 \downarrow$ \\
\hline 20 & PC(30:1) & $0.0103 \uparrow$ & PE(18:1/18:0) & 0.0745 & $\begin{array}{l}\operatorname{PE}(40: 2)+ \\
\operatorname{PC}(37: 2)^{5}\end{array}$ & $0.0002 \downarrow$ & Cer(d17:1/22:0) & $0.0435 \uparrow$ \\
\hline
\end{tabular}

aAbbreviations: ESI+, positive electrospray ionization mode in mass spectrometry. ESI-, negative electrospray ionization mode in mass spectrometry. Cer, ceramide. HexCer, hexosylceramide. SM, sphingomyelin. TG, triacylglycerol. DG, diacylglycerol. LysoPC, lysophophosphatidylcholine. LysoPE, lysophosphatidylethanolamine. PC, phosphatidylcholine. PE, phosphatidylethanolamine. PI, phosphatidylinositol. ${ }^{b} p$-values were obtained from repeated measures of ANOVA by the SAS software. "The bold text represents the species displaying significant diet or time effects in repeated measures ANOVA $(P<0.05)$. ${ }^{\mathrm{d}}$ The arrows $(\uparrow$ and $\downarrow)$ indicates the increase or decrease of lipid concentrations in the HIGH group compared to the CON group or the increase or decrease of lipid concentrations across the time, respectively. ${ }^{\mathrm{e}} \mathrm{The}$ ambiguous subspecies indicates that the lipidomic profiling gave two possible identifications of the lipid. 
Table 3 Top 20 lipids that contributed to the differences between the lipidomic profiles of HIGH and CON groups or that between different time points in the AT datasets.

\begin{tabular}{|c|c|c|c|c|}
\hline \multirow{2}{*}{ Rank } & \multicolumn{2}{|c|}{ AT ESI+ Diet (PL1) } & \multicolumn{2}{|c|}{ AT ESI- Diet (PL2) } \\
\hline & Lipid species & $P$-value & Lipid species & $P$-value \\
\hline 1 & $\mathrm{PC}(34: 2)$ & 0.2709 & $\operatorname{PE}(36: 3 e)$ & 0.2818 \\
\hline 2 & TG(56:5) & 0.7784 & $\mathrm{PI}(38: 5)$ & 0.3775 \\
\hline 3 & $\mathrm{TG}(58: 7)$ & 0.0843 & PI(18:0/20:4) & 0.298 \\
\hline 4 & $\mathrm{TG}(53: 2)$ & 0.903 & $\operatorname{Cer}(d 18: 1 / 20: 0)$ & $0.0243 \uparrow$ \\
\hline 5 & TG(17:0/18:1/18:1) & 0.6762 & $\operatorname{Cer}(d 17: 1 / 22: 0)$ & $0.0269 \uparrow$ \\
\hline 6 & $\mathrm{TG}(56: 5)$ & 0.1568 & $\begin{array}{l}\operatorname{PE}(16: 0 / 20: 4)+ \\
\operatorname{PE}(18: 2 / 18: 2)\end{array}$ & 0.1147 \\
\hline 7 & $\operatorname{SM}(d 18: 1 / 24: 0)$ & $0.0341 \downarrow$ & Cer(d18:1/18:0) & 0.8897 \\
\hline 8 & TG(47:3) & 0.2982 & $\operatorname{PE}(18: 0 / 18: 2)$ & 0.725 \\
\hline 9 & $\mathrm{TG}(52: 4)$ & 0.6726 & Cer(d18:1/22:0) & $0.0173 \uparrow$ \\
\hline 10 & $\mathrm{TG}(54: 5)$ & 0.1141 & $\operatorname{PE}(36: 3)$ & 0.5619 \\
\hline 11 & $\begin{array}{l}\text { TG(14:0/16:0/17:0)+ } \\
\text { TG(14:0/18:0/15:0) }\end{array}$ & 0.5382 & Cer(d18:1/16:0) & 0.3368 \\
\hline 12 & LysoPC(16:0) & 0.3203 & $\operatorname{PE}(18: 0 / 20: 3)$ & 0.6399 \\
\hline 13 & $\mathrm{TG}(51: 4)$ & 0.6832 & $\operatorname{PE}(36: 2)$ & 0.7443 \\
\hline 14 & ChoE(18:0) fragm & 0.0934 & $\operatorname{Cer}(\mathrm{d} 18: 1 / 22: 1)$ & 0.0749 \\
\hline 15 & $\mathrm{PC}(32: 1)$ & 0.7274 & $\operatorname{PE}(38: 4)$ & 0.6935 \\
\hline 16 & $\mathrm{TG}(50: 4)$ & 0.7212 & $\operatorname{PE}(38: 4)$ & 0.8571 \\
\hline 17 & SM(d18:1/24:1) & 0.6415 & $\mathrm{PI}(40: 5)$ & 0.1339 \\
\hline 18 & TG(48:4) & 0.8332 & $\operatorname{PE}(38: 4 \mathrm{e})$ & 0.1509 \\
\hline 19 & $\mathrm{PC}(38: 3)$ & 0.6716 & $\operatorname{Cer}(\mathrm{d} 18: 1 / 25: 0)$ & 0.5819 \\
\hline 20 & $\mathrm{PC}(36: 2)$ & 0.5518 & $\operatorname{PE}(36: 2 e)$ & 0.6256 \\
\hline
\end{tabular}

aAbbreviations: ESI+, positive electrospray ionization mode in mass spectrometry. ESI-, negative electrospray ionization mode in mass spectrometry Cer, ceramide. SM, sphingomyelin. TG, triacylglycerol. ChoE, cholesteryl ester, LysoPC lysophophosphatidylcholine. PC, phosphatidylcholine. PE, phosphatidylethanolamine. PI, phosphatidylinositol. ${ }^{\mathrm{b}} P$ values were obtained from repeated measures ANOVA in SAS. ${ }^{c}$ The bold text represents the species that have significant diet or time effect in repeated measures ANOVA $(P<0.05)$. ${ }^{\mathrm{d}}$ The arrows $(\uparrow$ and $\downarrow)$ indicate the increase or decrease of lipid concentrations in the HIGH group compared to the CON group or the increase or decrease of lipid concentrations

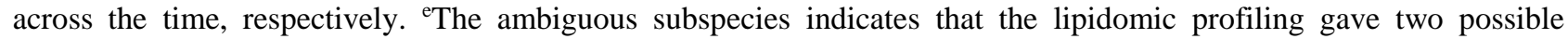
identifications of the lipid. 
Prepartal overfeeding alters the lipidomic profiles associated with insulin resistance in the liver and the adipose tissue of transition dairy cows

\section{Nanbing Qin*, Tuomo Kokkonen*, Siru Salin*, Tuulikki Seppänen-Laakso $†$, Juhani} Taponen $\neq$, AilaVanhatalo* and Kari Elo*

*Department of Agricultural Sciences, P.O. Box 28, FI-00014 University of Helsinki, Finland $\dagger$ Industrial Biotechnology, VTT Technical Research Centre of Finland Ltd, Tietotie 2, P.O. Box 1000, FI-02044 VTT, Finland

$\ddagger$ Department of Production Animal Medicine, University of Helsinki, Paroninkuja 20, FI-04920, Saarentaus, Finland

Corresponding author:

Kari Elo

kari.elo@helsinki.fi

Journal: Metabolomics 
571 Lipidomics runs were performed on a Waters Q-Tof Premier mass spectrometer fitted with an 572 Acquity Ultra Performance LC ${ }^{\mathrm{TM}}$ (UPLC). The column (at $50^{\circ} \mathrm{C}$ ) was an Acquity UPLC ${ }^{\mathrm{TM}}$ BEH $573 \mathrm{C} 182.1 \times 100 \mathrm{~mm}$ with $1.7 \mu \mathrm{m}$ particles. The solvent system included ultrapure water $(1 \% 1 \mathrm{M}$ $\left.574 \mathrm{NH}_{4} \mathrm{Ac}, 0.1 \% \mathrm{HCOOH}\right)$ and (B) acetonitrile/isopropanol (1:1, $1 \% 1 \mathrm{M} \mathrm{NH}_{4} \mathrm{Ac}, 0.1 \% \mathrm{HCOOH}, \mathrm{LC}-$ 575 MS grade). The gradient started from $65 \%$ A / 35\% B, reached $80 \%$ B in 2 min, $100 \%$ B in 7 min, 576 and remained there for $7 \mathrm{~min}$. The flow rate was $0.400 \mathrm{ml} / \mathrm{min}$ and the injected amount $2.0 \mu \mathrm{l}$ 577 (Acquity Sample Organizer, at $10^{\circ} \mathrm{C}$ ). Reserpine and leucine enkephaline were used as lock spray 578 reference compounds in ESI positive and negative ion modes, respectively.

580 The lipid profiling was carried out in either the ESI positive or ESI negative mode and the data 581 (centroid) were collected at a mass range of $\mathrm{m} / \mathrm{z}$ 300-1200 with scan duration of $0.2 \mathrm{sec}$. The cone 582 and capillary voltages were $40 \mathrm{~V}$ and $3.1 \mathrm{kV}$, respectively. Source temperature was $120{ }^{\circ} \mathrm{C}$ and the 583 desolvation gas flow and temperature $800 \mathrm{~L} / \mathrm{h}$ and $270{ }^{\circ} \mathrm{C}$, respectively. The peaks were measured 584 by using the peak heights. 


\section{Online Resource Table S1}

Number of masses detected in lipidomic profiling.

\begin{tabular}{lllll}
\hline \multirow{2}{*}{ Class } & \multicolumn{2}{c}{ Liver } & \multicolumn{2}{c}{ Adipose tissue } \\
\cline { 2 - 5 } & ESI+ & ESI- & ESI+ & ESI- \\
\hline Total detected masses & 296 & 1325 & 541 & 603 \\
Ceramide (Cer) & 6 & 25 & 0 & 16 \\
Hexosyl ceramide (HexCer) & 0 & 6 & 0 & 1 \\
Diacylglycerol (DG) & 2 & 0 & 0 & 0 \\
Phophosphatidylcholine (PC) & 69 & 8 & 7 & 4 \\
Phosphatidylethanolamine (PE) & 54 & 21 & 0 & 26 \\
Phosphatidylinositol (PI) & 1 & 1 & 0 & 6 \\
Sphingomyelin (SM) & 9 & 1 & 3 & 1 \\
Cholesteryl ester (CHoE) & 0 & 0 & 3 & 0 \\
Lysophophosphatidylcholine (LysoPC) & 3 & 1 & 2 & 0 \\
Lysophosphatidylethanolamine (Lyso PE) & 1 & 1 & 0 & 0 \\
Triacylglycerol (TG) & 117 & 0 & 40 & 0 \\
Unknown & 34 & 1261 & 486 & 549 \\
\hline Abbreviationyyyyyy
\end{tabular}

Abbreviations: ESI+, positive electrospray ionization mode in mass spectrometry. ESI-, negative electrospray ionization mode in mass spectrometry. 


\section{Online Resource Table S2}

PCA-LDA loadings of lipid subspecies identified in lipidomic profiling.

Formula for principal component analysis-linear discriminant analysis (PCA-LDA) functions $\mathrm{F}\left(\mathrm{PL}_{\mathrm{n}}\right)=\mathrm{PL}_{\mathrm{n}, 1} \cdot \mathrm{VAR}_{1}+\mathrm{PL}_{\mathrm{n}, 2} \cdot \mathrm{VAR}_{2}+\mathrm{PL}_{\mathrm{n}, 3} \cdot \mathrm{VAR}_{3}+\cdots+\mathrm{PL}_{\mathrm{n}, \mathrm{m}} \cdot \mathrm{VAR}_{\mathrm{m}}$ $\mathrm{F}\left(\mathrm{PL}_{\mathrm{n}}\right)=\mathrm{PCA}-\mathrm{LDA}$ function $\mathrm{n}, \mathrm{PL}=\mathrm{PCA}-\mathrm{LDA}$ loading $\mathrm{n}, \mathrm{VAR}=$ concentration of lipid, $\mathrm{m}=$ total number of lipid subspecies, $\mathrm{n}=$ total number of PCA-LDA funcitons

a) Liver positive electrospray ionization mode (ESI+)

\begin{tabular}{|c|c|c|c|c|}
\hline No. & Subspecies & PL1 & PL2 & PL3 \\
\hline 1 & Cer(d18:1/16:0) & -0.029 & -0.067 & -0.011 \\
\hline 2 & Cer(d18:1/18:0) & -0.038 & -0.101 & -0.029 \\
\hline 3 & Cer(d18:1/24:0) & -0.024 & -0.049 & 0.002 \\
\hline 4 & $\operatorname{Cer}(\mathrm{d} 18: 1 / 24: 1)$ & -0.039 & -0.087 & 0.020 \\
\hline 5 & Cer(d18:1/26:1) & -0.001 & 0.039 & 0.019 \\
\hline 6 & Cer(d18:2/23:0) & -0.051 & -0.049 & 0.020 \\
\hline 7 & DG(33:5) & 0.068 & 0.069 & -0.015 \\
\hline 8 & $\mathrm{DG}(34: 1) \mathrm{Na}$ & 0.022 & 0.030 & -0.010 \\
\hline 9 & LysoPC(16:0) & -0.027 & -0.130 & -0.044 \\
\hline 10 & LysoPC(18:0) & -0.027 & -0.153 & -0.036 \\
\hline 11 & LysoPC(18:2) & 0.023 & -0.028 & -0.042 \\
\hline 12 & LysoPE(18:0) & -0.023 & -0.149 & -0.025 \\
\hline 13 & $\mathrm{PC}(16: 0 / 20: 4)$ & -0.024 & 0.062 & -0.007 \\
\hline 14 & $\mathrm{PC}(16: 0 / 20: 5)$ & 0.017 & -0.008 & -0.046 \\
\hline 15 & $\mathrm{PC}(16: 0 / 22: 5)$ & -0.003 & -0.064 & -0.050 \\
\hline 16 & $\operatorname{PC}(16: 1 / 20: 4)$ & 0.017 & -0.045 & 0.006 \\
\hline 17 & $\mathrm{PC}(18: 0 / 22: 6)$ & -0.058 & -0.014 & -0.021 \\
\hline 18 & PC(18:1/20:4) & -0.034 & -0.049 & -0.047 \\
\hline 19 & $\mathrm{PC}(30: 0)$ & 0.000 & -0.070 & -0.024 \\
\hline 20 & $\mathrm{PC}(30: 1)$ & 0.018 & -0.110 & -0.027 \\
\hline 21 & $\mathrm{PC}(32: 0)$ & 0.029 & 0.010 & 0.018 \\
\hline 22 & $\mathrm{PC}(32: 1)$ & 0.035 & -0.017 & -0.038 \\
\hline 23 & $\mathrm{PC}(32: 1)$ & -0.015 & -0.104 & 0.001 \\
\hline 24 & $\mathrm{PC}(32: 1 \mathrm{e})$ & -0.019 & -0.029 & 0.027 \\
\hline 25 & $\mathrm{PC}(32: 2)$ & 0.030 & -0.091 & -0.028 \\
\hline 26 & $\mathrm{PC}(32: 2)$ & -0.009 & -0.054 & -0.018 \\
\hline 27 & $\mathrm{PC}(32: 2)$ & 0.027 & -0.137 & -0.046 \\
\hline 28 & $\mathrm{PC}(32: 2 \mathrm{e})$ & 0.019 & -0.118 & -0.028 \\
\hline 29 & $\mathrm{PC}(33: 2)$ & 0.022 & -0.041 & -0.007 \\
\hline 30 & $\mathrm{PC}(34: 1)$ & 0.035 & 0.063 & -0.026 \\
\hline 31 & $\operatorname{PC}(34: 1 \mathrm{e})$ & 0.029 & -0.006 & 0.012 \\
\hline 32 & $\mathrm{PC}(34: 2)$ & 0.032 & 0.028 & 0.001 \\
\hline 33 & $\mathrm{PC}(34: 2)$ & 0.018 & -0.125 & -0.035 \\
\hline 34 & $\mathrm{PC}(34: 3)$ & -0.003 & -0.065 & -0.004 \\
\hline 35 & $\mathrm{PC}(34: 3)$ & 0.052 & 0.059 & 0.014 \\
\hline 36 & $\mathrm{PC}(34: 4)$ & 0.047 & -0.006 & 0.004 \\
\hline 37 & $\mathrm{PC}(34: 4)+\mathrm{PE}(37: 4)^{\mathrm{a}}$ & 0.021 & 0.091 & 0.033 \\
\hline 38 & $\mathrm{PC}(34: 4 \mathrm{e})$ & 0.037 & 0.020 & 0.011 \\
\hline 39 & $\mathrm{PC}(34: 5)$ & -0.039 & -0.074 & 0.005 \\
\hline 40 & $\mathrm{PC}(35: 2)$ & 0.017 & -0.030 & 0.020 \\
\hline 41 & $\mathrm{PC}(36: 1)$ & 0.028 & -0.007 & -0.024 \\
\hline 42 & $\mathrm{PC}(36: 2)$ & 0.011 & -0.018 & 0.016 \\
\hline 43 & $\mathrm{PC}(36: 2)$ & 0.038 & 0.026 & 0.022 \\
\hline 44 & $\mathrm{PC}(36: 3)$ & -0.001 & -0.009 & 0.019 \\
\hline 45 & $\mathrm{PC}(36: 4)$ & -0.046 & 0.010 & 0.044 \\
\hline 46 & $\mathrm{PC}(36: 4 \mathrm{e})$ & 0.045 & 0.084 & 0.003 \\
\hline 47 & $\mathrm{PC}(36: 5)$ & -0.019 & 0.008 & 0.006 \\
\hline
\end{tabular}




\begin{tabular}{|c|c|c|c|c|}
\hline 48 & $\mathrm{PC}(36: 6)$ & 0.001 & -0.079 & -0.003 \\
\hline 49 & $\mathrm{PC}(36: 6 e)$ & 0.029 & -0.082 & -0.088 \\
\hline 50 & $\mathrm{PC}(38: 1)$ & 0.000 & 0.051 & 0.013 \\
\hline 51 & $\mathrm{PC}(38: 1)$ & 0.012 & -0.029 & -0.012 \\
\hline 52 & $\mathrm{PC}(38: 1)$ & -0.035 & -0.049 & -0.006 \\
\hline 53 & $\mathrm{PC}(38: 3)$ & -0.047 & -0.001 & 0.034 \\
\hline 54 & $\mathrm{PC}(38: 3)$ & -0.031 & 0.065 & 0.031 \\
\hline 55 & $\mathrm{PC}(38: 3)$ & -0.012 & 0.108 & 0.028 \\
\hline 56 & $\mathrm{PC}(38: 4)$ & -0.046 & 0.005 & 0.026 \\
\hline 57 & $\mathrm{PC}(38: 4)$ & 0.010 & 0.116 & 0.011 \\
\hline 58 & $\mathrm{PC}(38: 4 \mathrm{e})$ & -0.046 & -0.022 & 0.006 \\
\hline 59 & $\mathrm{PC}(38: 5 \mathrm{e})$ & 0.034 & 0.039 & 0.007 \\
\hline 60 & $\mathrm{PC}(38: 5 \mathrm{e})$ & -0.011 & 0.005 & 0.001 \\
\hline 61 & $\mathrm{PC}(38: 5 \mathrm{e})$ & -0.018 & -0.045 & 0.012 \\
\hline 62 & $\mathrm{PC}(38: 6)$ & 0.059 & -0.028 & -0.018 \\
\hline 63 & $\mathrm{PC}(38: 6)$ & -0.024 & 0.018 & -0.014 \\
\hline 64 & $\mathrm{PC}(38: 6 e)$ & -0.028 & -0.057 & 0.015 \\
\hline 65 & $\mathrm{PC}(38: 7 \mathrm{e})$ & -0.044 & 0.010 & 0.040 \\
\hline 66 & $\mathrm{PC}(40: 3)$ & -0.028 & 0.024 & 0.004 \\
\hline 67 & $\mathrm{PC}(40: 3 \mathrm{e})$ & 0.041 & 0.080 & 0.006 \\
\hline 68 & $\mathrm{PC}(40: 4)$ & 0.000 & -0.025 & -0.039 \\
\hline 69 & PC $(40: 4)$ & -0.042 & -0.025 & -0.008 \\
\hline 70 & $\mathrm{PC}(40: 4 \mathrm{e})$ & 0.021 & -0.003 & 0.027 \\
\hline 71 & PC(40:5) & -0.017 & 0.008 & -0.007 \\
\hline 72 & $\mathrm{PC}(40: 5 \mathrm{e})$ & 0.015 & 0.044 & 0.005 \\
\hline 73 & PC(40:7) & 0.006 & -0.067 & 0.011 \\
\hline 74 & PC(40:7) & -0.007 & 0.059 & 0.025 \\
\hline 75 & $\mathrm{PC}(40: 8)$ & 0.069 & 0.047 & 0.003 \\
\hline 76 & $\mathrm{PC}(40: 8 \mathrm{e})$ & -0.005 & 0.017 & 0.021 \\
\hline 77 & PC(p16:0/20:4) & 0.013 & 0.002 & -0.019 \\
\hline 78 & $\operatorname{PE}(32: 1)$ & 0.012 & -0.175 & -0.010 \\
\hline 79 & $\operatorname{PE}(32: 3 e)$ & 0.035 & -0.076 & 0.000 \\
\hline 80 & $\operatorname{PE}(34: 1)$ & 0.035 & 0.027 & -0.005 \\
\hline 81 & $\operatorname{PE}(34: 2)$ & 0.027 & 0.003 & 0.020 \\
\hline 82 & $\mathrm{PE}(34: 2 \mathrm{e})$ & 0.028 & -0.145 & -0.045 \\
\hline 83 & $\operatorname{PE}(34: 3 e)$ & 0.020 & -0.088 & 0.003 \\
\hline 84 & $\operatorname{PE}(34: 4 e)$ & -0.005 & -0.114 & 0.006 \\
\hline 85 & $\operatorname{PE}(36: 0)$ & -0.014 & -0.022 & 0.004 \\
\hline 86 & $\operatorname{PE}(36: 0)$ & 0.059 & 0.141 & 0.020 \\
\hline 87 & $\operatorname{PE}(36: 1)$ & 0.004 & 0.019 & 0.029 \\
\hline 88 & $\operatorname{PE}(36: 1)$ & 0.029 & -0.002 & -0.019 \\
\hline 89 & $\operatorname{PE}(36: 1)$ & 0.004 & 0.006 & -0.006 \\
\hline 90 & $\operatorname{PE}(36: 2)$ & 0.020 & 0.005 & 0.025 \\
\hline 91 & $\operatorname{PE}(36: 2 e)$ & 0.064 & 0.136 & -0.025 \\
\hline 92 & $\operatorname{PE}(36: 2 e)$ & 0.002 & -0.006 & 0.018 \\
\hline 93 & $\operatorname{PE}(36: 2 e)$ & -0.010 & -0.083 & -0.001 \\
\hline 94 & $\operatorname{PE}(36: 3)$ & 0.002 & -0.059 & 0.014 \\
\hline 95 & $\operatorname{PE}(36: 3 e)$ & -0.008 & -0.018 & 0.051 \\
\hline 96 & $\operatorname{PE}(36: 3 e)$ & 0.007 & -0.070 & 0.012 \\
\hline 97 & $\operatorname{PE}(36: 4)$ & 0.034 & 0.091 & 0.028 \\
\hline 98 & $\operatorname{PE}(36: 4 e)$ & -0.028 & -0.041 & 0.022 \\
\hline 99 & $\operatorname{PE}(36: 5)$ & 0.031 & 0.044 & 0.029 \\
\hline 100 & $\operatorname{PE}(36: 5 e)$ & -0.046 & 0.075 & 0.068 \\
\hline 101 & $\operatorname{PE}(36: 5 e)$ & 0.027 & 0.117 & 0.019 \\
\hline 102 & $\operatorname{PE}(38: 0)$ & -0.018 & -0.026 & 0.004 \\
\hline 103 & $\operatorname{PE}(38: 1)$ & -0.006 & -0.026 & 0.005 \\
\hline 104 & $\operatorname{PE}(38: 1)$ & -0.009 & -0.028 & -0.008 \\
\hline
\end{tabular}




\begin{tabular}{|c|c|c|c|c|}
\hline 105 & $\operatorname{PE}(38: 1 \mathrm{e})$ & 0.024 & 0.048 & 0.022 \\
\hline 106 & $\operatorname{PE}(38: 2)$ & 0.022 & -0.053 & 0.000 \\
\hline 107 & $\operatorname{PE}(38: 2)$ & -0.048 & 0.092 & 0.053 \\
\hline 108 & $\operatorname{PE}(38: 2)$ & -0.024 & 0.028 & 0.010 \\
\hline 109 & $\operatorname{PE}(38: 3)$ & -0.014 & 0.130 & 0.058 \\
\hline 110 & $\operatorname{PE}(38: 3)$ & -0.039 & 0.096 & 0.049 \\
\hline 111 & $\operatorname{PE}(38: 3)$ & -0.043 & 0.059 & 0.043 \\
\hline 112 & $\operatorname{PE}(38: 4)$ & 0.015 & 0.093 & 0.026 \\
\hline 113 & $\operatorname{PE}(38: 4)$ & -0.047 & 0.026 & 0.036 \\
\hline 114 & $\operatorname{PE}(38: 5)$ & 0.016 & 0.057 & 0.012 \\
\hline 115 & $\operatorname{PE}(38: 5)$ & 0.020 & 0.041 & 0.005 \\
\hline 116 & $\operatorname{PE}(38: 5 \mathrm{e})$ & -0.031 & 0.074 & 0.041 \\
\hline 117 & $\operatorname{PE}(38: 6)$ & 0.020 & -0.009 & 0.046 \\
\hline 118 & $\operatorname{PE}(38: 6 \mathrm{e})$ & 0.040 & 0.057 & 0.031 \\
\hline 119 & $\operatorname{PE}(38: 6 e)$ & -0.013 & -0.088 & 0.024 \\
\hline 120 & $\mathrm{PE}(40.1)+\mathrm{PC}(37: 1)^{\mathrm{a}}$ & -0.039 & -0.083 & -0.013 \\
\hline 121 & $\operatorname{PE}(40: 2)+\mathrm{PC}(37: 2)^{\mathrm{a}}$ & -0.047 & -0.081 & -0.008 \\
\hline 122 & $\operatorname{PE}(40: 3)$ & 0.008 & 0.061 & 0.014 \\
\hline 123 & $\operatorname{PE}(40: 3)$ & -0.040 & -0.005 & 0.029 \\
\hline 124 & $\operatorname{PE}(40: 4)$ & -0.001 & 0.056 & 0.004 \\
\hline 125 & $\operatorname{PE}(40: 4 \mathrm{e})$ & 0.066 & 0.039 & 0.020 \\
\hline 126 & $\operatorname{PE}(40: 5)$ & -0.023 & 0.025 & -0.007 \\
\hline 127 & $\operatorname{PC}(37: 5)$ & -0.014 & -0.119 & -0.041 \\
\hline 128 & $\operatorname{PE}(40: 6)$ & -0.034 & -0.019 & -0.038 \\
\hline 129 & $\operatorname{PE}(40: 6)$ & 0.015 & -0.043 & 0.004 \\
\hline 130 & $\operatorname{PE}(40: 6)$ & 0.001 & 0.036 & 0.019 \\
\hline 131 & $\operatorname{PE}(40: 6)$ & -0.015 & 0.124 & 0.019 \\
\hline 132 & PE(40:6e) & -0.029 & 0.078 & 0.010 \\
\hline 133 & $\mathrm{PC}(39: 6)$ & 0.026 & 0.085 & 0.018 \\
\hline 134 & $\mathrm{PE}(\mathrm{p} 16: 0 / 22: 6)$ & -0.084 & -0.094 & 0.014 \\
\hline 135 & PE(p18:0/20:4) & -0.013 & 0.166 & 0.028 \\
\hline 136 & $\operatorname{PI}(38: 5)$ & 0.043 & -0.044 & -0.011 \\
\hline 137 & $\operatorname{SM}(d 18: 1 / 14: 0)$ & -0.001 & -0.118 & -0.017 \\
\hline 138 & $\operatorname{SM}(d 18: 1 / 16: 0)$ & -0.056 & -0.051 & 0.023 \\
\hline 139 & $\operatorname{SM}(d 18: 1 / 16: 1)$ & -0.046 & -0.061 & 0.008 \\
\hline 140 & $\operatorname{SM}(d 18: 1 / 18: 0)$ & -0.039 & -0.074 & 0.019 \\
\hline 141 & $\operatorname{SM}(\mathrm{d} 18: 1 / 20: 0)$ & -0.022 & -0.061 & 0.031 \\
\hline 142 & $\operatorname{SM}(d 18: 1 / 21: 0)$ & -0.005 & -0.082 & 0.036 \\
\hline 143 & $\operatorname{SM}(d 18: 1 / 23: 0)$ & -0.029 & -0.087 & 0.037 \\
\hline 144 & $\operatorname{SM}(d 18: 1 / 23: 1)$ & -0.039 & -0.086 & 0.029 \\
\hline 145 & $\operatorname{SM}(d 18: 1 / 24: 1)$ & 0.010 & -0.074 & -0.030 \\
\hline \multirow[t]{2}{*}{146} & TG(14:0/16:0/16:0)+ & 0.035 & -0.008 & -0.015 \\
\hline & $\operatorname{TG}(16: 0 / 18: 0 / 12: 0)^{\mathrm{a}}$ & & & \\
\hline \multirow[t]{2}{*}{147} & TG(14:0/16:0/17:0)+ & 0.038 & -0.019 & -0.015 \\
\hline & $\mathrm{TG}(14: 0 / 18: 0 / 15: 0)^{\mathrm{a}}$ & & & \\
\hline \multirow[t]{2}{*}{148} & TG(14:0/16:0/17:0)+ & 0.044 & -0.006 & -0.012 \\
\hline & $\mathrm{TG}(14: 0 / 18: 0 / 15: 0)^{\mathrm{a}}$ & & & \\
\hline \multirow[t]{2}{*}{149} & $\mathrm{TG}(16: 0 / 16: 0 / 16: 0)+$ & 0.024 & -0.007 & -0.008 \\
\hline & $\mathrm{TG}(14: 0 / 16: 0 / 18: 0)^{\mathrm{a}}$ & & & \\
\hline 150 & TG(16:0/18:1/18:1) & 0.000 & -0.030 & -0.009 \\
\hline \multirow[t]{2}{*}{151} & TG(16:0/18:1/20:1)+ & -0.028 & -0.035 & -0.003 \\
\hline & $\mathrm{TG}(18: 0 / 18: 1 / 18: 1)^{\mathrm{a}}$ & & & \\
\hline \multirow[t]{2}{*}{152} & $\mathrm{TG}(16: 0 / 18: 2 / 18: 1)+$ & 0.014 & -0.019 & 0.000 \\
\hline & $\mathrm{TG}(16: 1 / 18: 1 / 18: 1)^{\mathrm{a}}$ & & & \\
\hline 153 & $\mathrm{TG}(17: 1 / 18: 1 / 18: 2)$ & 0.022 & -0.029 & 0.000 \\
\hline
\end{tabular}




\begin{tabular}{|c|c|c|c|c|}
\hline 154 & $\begin{array}{l}\text { TG(18:1/16:1/18:2)+ } \\
\text { TG(18:2/18:2/16:0) }\end{array}$ & 0.022 & -0.013 & 0.003 \\
\hline 155 & TG(18:1/18:1/18:1) & -0.008 & -0.023 & 0.007 \\
\hline 156 & $\mathrm{TG}(18: 1 / 18: 1 / 22: 1)+$ & 0.019 & -0.038 & 0.001 \\
\hline & $\mathrm{TG}(20: 1 / 20: 1 / 18: 1)^{\mathrm{a}}$ & & & \\
\hline 157 & $\mathrm{TG}(37: 0)$ & 0.015 & 0.019 & 0.010 \\
\hline 158 & $\mathrm{TG}(38: 0)$ & -0.018 & -0.026 & 0.016 \\
\hline 159 & $\mathrm{TG}(42: 0)$ & 0.028 & -0.038 & -0.009 \\
\hline 160 & $\mathrm{TG}(42: 1)$ & 0.028 & -0.016 & -0.011 \\
\hline 161 & $\mathrm{TG}(44: 0)$ & 0.040 & -0.001 & -0.016 \\
\hline 162 & $\mathrm{TG}(44: 1)$ & 0.039 & -0.002 & -0.007 \\
\hline 163 & $\mathrm{TG}(44: 2)$ & 0.035 & -0.018 & -0.006 \\
\hline 164 & $\mathrm{TG}(45: 0)$ & 0.054 & 0.002 & -0.015 \\
\hline 165 & $\mathrm{TG}(46: 1)$ & 0.041 & 0.010 & -0.008 \\
\hline 166 & $\mathrm{TG}(46: 2)$ & 0.035 & -0.019 & -0.006 \\
\hline 167 & TG(46:3) & 0.009 & 0.001 & 0.022 \\
\hline 168 & TG(46:3) & 0.044 & -0.010 & -0.002 \\
\hline 169 & $\mathrm{TG}(47: 1)$ & 0.045 & 0.010 & -0.007 \\
\hline 170 & $\mathrm{TG}(47: 2)$ & 0.046 & -0.003 & -0.005 \\
\hline 171 & $\mathrm{TG}(47: 3)$ & 0.049 & -0.001 & -0.004 \\
\hline 172 & $\mathrm{TG}(48: 1)$ & 0.030 & 0.008 & -0.004 \\
\hline 173 & $\mathrm{TG}(48: 2)$ & 0.032 & -0.002 & -0.002 \\
\hline 174 & $\mathrm{TG}(48: 3)$ & 0.041 & -0.004 & -0.001 \\
\hline 175 & TG(48:4) & 0.039 & -0.006 & 0.001 \\
\hline 176 & $\mathrm{TG}(48: 5)$ & 0.070 & 0.147 & 0.010 \\
\hline 177 & $\mathrm{TG}(49: 0)$ & 0.017 & -0.003 & -0.007 \\
\hline 178 & $\mathrm{TG}(49: 0)$ & 0.022 & 0.003 & 0.001 \\
\hline 179 & $\mathrm{TG}(49: 1)$ & 0.034 & 0.011 & -0.002 \\
\hline 180 & $\mathrm{TG}(49: 2)$ & 0.036 & 0.005 & 0.001 \\
\hline 181 & $\mathrm{TG}(49: 3)$ & 0.042 & -0.004 & 0.000 \\
\hline 182 & $\mathrm{TG}(49: 4)$ & 0.042 & -0.011 & -0.002 \\
\hline 183 & $\mathrm{TG}(50: 0)$ & 0.037 & 0.017 & 0.001 \\
\hline 184 & TG(50:0) & 0.002 & 0.002 & -0.008 \\
\hline 185 & TG(50:1) & 0.038 & 0.000 & -0.001 \\
\hline 186 & TG(50:1) & 0.017 & 0.004 & -0.012 \\
\hline 187 & $\mathrm{TG}(50: 2)$ & -0.006 & 0.014 & -0.006 \\
\hline 188 & $\mathrm{TG}(50: 2)$ & 0.037 & 0.007 & 0.000 \\
\hline 189 & $\mathrm{TG}(50: 2)$ & 0.022 & -0.002 & 0.001 \\
\hline 190 & $\mathrm{TG}(50: 3)$ & 0.035 & 0.004 & 0.002 \\
\hline 191 & TG(50:6) & 0.029 & -0.013 & 0.012 \\
\hline 192 & $\mathrm{TG}(51: 1)$ & 0.021 & -0.033 & 0.004 \\
\hline 193 & $\mathrm{TG}(51: 1)$ & 0.013 & 0.003 & 0.000 \\
\hline 194 & $\mathrm{TG}(51: 1)$ & 0.022 & 0.015 & 0.002 \\
\hline 195 & $\mathrm{TG}(51: 2)$ & 0.021 & -0.001 & 0.002 \\
\hline 196 & $\mathrm{TG}(51: 2)$ & 0.029 & 0.016 & -0.001 \\
\hline 197 & $\mathrm{TG}(51: 3)$ & 0.032 & 0.003 & 0.004 \\
\hline 198 & TG(51:4) & 0.033 & -0.016 & 0.000 \\
\hline 199 & TG(51:5) & 0.053 & 0.030 & -0.026 \\
\hline 200 & TG(51:5) & 0.039 & -0.004 & 0.006 \\
\hline 201 & TG(52:0) & -0.045 & 0.038 & 0.013 \\
\hline 202 & TG(52:0) & 0.031 & 0.029 & 0.004 \\
\hline 203 & TG(52:1) & 0.000 & 0.018 & -0.005 \\
\hline 204 & TG(52:3) & 0.012 & 0.017 & -0.041 \\
\hline 205 & TG(52:5) & 0.028 & -0.006 & 0.006 \\
\hline 206 & TG(53:0) & -0.013 & 0.003 & 0.001 \\
\hline 207 & TG(53:0) & -0.039 & 0.010 & 0.018 \\
\hline 208 & $\mathrm{TG}(53: 0)$ & 0.029 & 0.024 & 0.012 \\
\hline
\end{tabular}




\begin{tabular}{|c|c|c|c|c|}
\hline 209 & TG(53:0) & -0.041 & 0.018 & 0.014 \\
\hline 210 & TG(53:1) & -0.019 & -0.008 & 0.002 \\
\hline 211 & TG(53:1) & 0.029 & -0.011 & 0.001 \\
\hline 212 & TG(53:1) & -0.010 & -0.001 & -0.005 \\
\hline 213 & TG(53:3) & 0.020 & -0.018 & 0.004 \\
\hline 214 & TG(53:3) & 0.016 & -0.001 & 0.005 \\
\hline 215 & TG(53:5) & 0.018 & 0.016 & 0.003 \\
\hline 216 & TG(53:5) & 0.028 & -0.019 & 0.005 \\
\hline 217 & TG(53:6) & 0.000 & -0.008 & 0.002 \\
\hline 218 & TG(54:0) & -0.058 & 0.039 & 0.023 \\
\hline 219 & $\mathrm{TG}(54: 0)$ & 0.023 & 0.015 & 0.003 \\
\hline 220 & TG $(54: 1)$ & -0.042 & 0.018 & 0.008 \\
\hline 221 & TG(54:1) & 0.027 & 0.001 & 0.003 \\
\hline 222 & TG(54:2) & 0.017 & 0.019 & 0.017 \\
\hline 223 & TG(54:2) & -0.006 & -0.002 & 0.011 \\
\hline 224 & TG(54:4) & 0.020 & 0.005 & 0.012 \\
\hline 225 & TG(54:5) & 0.023 & -0.005 & 0.008 \\
\hline 226 & TG(54:6) & 0.025 & -0.019 & 0.009 \\
\hline 227 & TG(54:9) & -0.003 & -0.004 & 0.006 \\
\hline 228 & $\mathrm{TG}(55: 1)$ & -0.003 & -0.008 & 0.005 \\
\hline 229 & TG(55:1) & 0.022 & -0.006 & 0.015 \\
\hline 230 & TG(55:2) & 0.027 & -0.013 & 0.002 \\
\hline 231 & TG(55:2) & -0.009 & -0.017 & 0.001 \\
\hline 232 & $\mathrm{TG}(55: 3)$ & -0.003 & -0.007 & 0.012 \\
\hline 233 & TG(55:4) & 0.017 & 0.002 & 0.023 \\
\hline 234 & TG(55:5) & 0.025 & -0.022 & 0.002 \\
\hline 235 & TG(55:6) & 0.028 & -0.036 & 0.005 \\
\hline 236 & TG(56:0) & 0.015 & 0.026 & 0.000 \\
\hline 237 & TG(56:1) & -0.007 & 0.011 & 0.014 \\
\hline 238 & TG(56:1) & 0.006 & 0.014 & 0.009 \\
\hline 239 & TG(56:1) & 0.023 & 0.017 & 0.013 \\
\hline 240 & TG(56:2) & -0.008 & -0.007 & 0.012 \\
\hline 241 & TG(56:2) & 0.029 & 0.008 & 0.006 \\
\hline 242 & TG(56:3) & 0.000 & 0.013 & 0.027 \\
\hline 243 & $\mathrm{TG}(56: 3)$ & -0.003 & -0.010 & 0.018 \\
\hline 244 & TG(56:3) & 0.002 & -0.078 & 0.004 \\
\hline 245 & TG(56:4) & 0.025 & -0.002 & 0.008 \\
\hline 246 & TG(56:4) & -0.008 & -0.015 & 0.029 \\
\hline 247 & $\mathrm{TG}(56: 4)$ & 0.002 & -0.036 & 0.013 \\
\hline 248 & TG(56:5) & 0.007 & -0.022 & 0.010 \\
\hline 249 & TG(56:5) & -0.001 & -0.008 & 0.024 \\
\hline 250 & TG(56:6) & 0.016 & -0.012 & 0.013 \\
\hline 251 & TG(56:7) & 0.016 & -0.037 & 0.011 \\
\hline 252 & TG(56:8) & 0.014 & -0.037 & 0.010 \\
\hline 253 & TG(57:1) & 0.023 & 0.019 & 0.010 \\
\hline 254 & $\mathrm{TG}(57: 2)$ & 0.021 & -0.010 & -0.003 \\
\hline 255 & $\mathrm{TG}(57: 3)$ & 0.029 & -0.014 & 0.003 \\
\hline 256 & $\mathrm{TG}(58: 2)$ & 0.024 & 0.005 & 0.005 \\
\hline 257 & TG(58:5) & 0.001 & -0.043 & 0.017 \\
\hline 258 & TG(58:5) & -0.043 & -0.056 & -0.011 \\
\hline 259 & TG(58:6) & -0.026 & -0.110 & 0.001 \\
\hline 260 & TG(58:7) & -0.002 & -0.083 & 0.012 \\
\hline 261 & TG(58:8) & -0.012 & -0.081 & 0.016 \\
\hline 262 & TG(59:3) & 0.000 & -0.041 & 0.007 \\
\hline
\end{tabular}


b) Liver negative electrospray ionization mode (ESI-)

\begin{tabular}{|c|c|c|c|c|}
\hline VAR & Species & PL1 & PL2 & PL3 \\
\hline$\overline{1}$ & $\overline{C e r(d 17: 1 / 22: 0)}$ & 0.200 & -0.053 & -0.008 \\
\hline 2 & Cer(d18:1/23:1) & -0.177 & 0.031 & -0.109 \\
\hline 3 & Cer(d18:0/16:0) & 0.034 & -0.074 & 0.090 \\
\hline 4 & Cer(d18:0/18:0) & -0.108 & -0.032 & -0.071 \\
\hline 5 & Cer(d18:0/23:0) & 0.073 & -0.099 & -0.081 \\
\hline 6 & $\operatorname{Cer}(\mathrm{d} 18: 0 / 24: 0)$ & -0.034 & -0.153 & -0.032 \\
\hline 7 & Cer(d18:1/16:0) & 0.089 & 0.086 & 0.071 \\
\hline 8 & Cer(d18:1/18:0) & -0.073 & 0.045 & 0.056 \\
\hline 9 & Cer(d18:1/19:0) & 0.083 & -0.004 & -0.053 \\
\hline 10 & Cer(d18:1/20:0) & 0.043 & -0.094 & -0.055 \\
\hline 11 & Cer(d18:1/22:0) & 0.117 & -0.120 & -0.055 \\
\hline 12 & Cer(d18:1/23:0) & 0.622 & 0.215 & -0.124 \\
\hline 13 & Cer(d18:1/23:0) & 0.239 & 0.004 & -0.120 \\
\hline 14 & Cer(d18:1/24:0) & -0.139 & -0.158 & -0.096 \\
\hline 15 & Cer(d18:1/24:1) & 0.175 & 0.096 & -0.127 \\
\hline 16 & Cer(d18:1/25:0) & -0.271 & -0.113 & -0.158 \\
\hline 17 & Cer(d18:1/26:0) & -0.081 & 0.127 & 0.087 \\
\hline 18 & Cer(d18:2/26:0) & -0.356 & -0.052 & -0.021 \\
\hline 19 & Cer(d18:2/16:0) & -0.113 & 0.092 & 0.076 \\
\hline 20 & Cer(d18:2/18:0) & 0.075 & 0.172 & -0.021 \\
\hline 21 & Cer(d18:2/20:0) & -0.325 & -0.040 & -0.046 \\
\hline 22 & Cer(d18:2/22:0) & -0.328 & -0.081 & -0.039 \\
\hline 23 & Cer(d18:2/23:1) & -0.159 & 0.076 & -0.035 \\
\hline 24 & Cer(d18:2/24:1) & 0.074 & 0.165 & -0.115 \\
\hline 25 & Cer(d18:1/25:1) & 0.087 & 0.098 & -0.057 \\
\hline 26 & Cer(d18:2/26:1) & -0.258 & -0.009 & -0.046 \\
\hline 27 & HexCer(d18:1/16:0) & -0.034 & -0.043 & 0.058 \\
\hline 28 & HexCer(d18:1/22:0) & -0.145 & -0.134 & 0.002 \\
\hline 29 & HexCer(d18:1/23:0) & 0.093 & -0.058 & 0.044 \\
\hline 30 & HexCer(d18:1/24:0) & -0.043 & 0.012 & 0.025 \\
\hline 31 & HexCer(d18:1/24:1) & -0.161 & 0.035 & -0.059 \\
\hline 32 & HexCer(d18:2/20:0) & -0.317 & -0.035 & 0.028 \\
\hline 33 & LysoPC(18:0) & 0.644 & 0.085 & 0.290 \\
\hline 34 & LysoPE(18:0) & 0.159 & 0.075 & 0.026 \\
\hline \multirow[t]{2}{*}{35} & $\mathrm{PC}(16: 0 / 16: 1)+$ & 0.026 & 0.105 & -0.071 \\
\hline & $\operatorname{PC}(14: 0 / 18: 1)^{\mathrm{a}}$ & & & \\
\hline 36 & $\operatorname{PC}(16: 0 / 20: 4)$ & -0.209 & 0.010 & -0.078 \\
\hline 37 & PC(18:1/20:1) & -0.083 & 0.062 & -0.051 \\
\hline 38 & $\mathrm{PC}(30: 0)$ & 0.115 & 0.019 & 0.031 \\
\hline 39 & $\mathrm{PC}(33: 0)$ & -0.134 & 0.045 & 0.089 \\
\hline 40 & $\mathrm{PC}(33: 1)$ & 0.696 & 0.490 & 0.034 \\
\hline 41 & $\mathrm{PC}(36: 2)$ & 0.167 & 0.202 & -0.018 \\
\hline 42 & $\mathrm{PC}(38: 3)$ & -0.441 & -0.208 & 0.035 \\
\hline \multirow[t]{2}{*}{43} & $\operatorname{PE}(16: 0 / 20: 4)+$ & -0.049 & -0.133 & 0.049 \\
\hline & $\operatorname{PE}(18: 2 / 18: 2)^{\mathrm{a}}$ & & & \\
\hline 44 & $\operatorname{PE}(18: 0 / 18: 1)$ & 0.046 & 0.055 & 0.038 \\
\hline 45 & $\mathrm{PE}(18: 0 / 20: 3)$ & -0.240 & -0.061 & 0.036 \\
\hline 46 & $\mathrm{PE}(18: 0 / 22: 4)$ & -0.086 & 0.085 & 0.057 \\
\hline 47 & PE(18:1/18:0) & 0.095 & 0.104 & 0.073 \\
\hline 48 & $\mathrm{PE}(32: 0)$ & 0.225 & 0.130 & 0.054 \\
\hline 49 & $\operatorname{PE}(34: 1)$ & 0.081 & -0.080 & 0.079 \\
\hline 50 & $\mathrm{PE}(36: 2)$ & 0.248 & 0.050 & -0.004 \\
\hline 51 & $\operatorname{PE}(36: 2 e)$ & 0.064 & 0.076 & -0.022 \\
\hline 52 & $\operatorname{PE}(36: 3)$ & 0.293 & 0.075 & 0.011 \\
\hline 53 & $\mathrm{PE}(36: 3 e)$ & 0.134 & -0.014 & -0.064 \\
\hline
\end{tabular}




\begin{tabular}{llrrr}
54 & $\mathrm{PE}(38: 4)$ & -0.207 & -0.056 & 0.041 \\
55 & $\mathrm{PE}(38: 4 \mathrm{e})$ & -0.156 & -0.024 & 0.041 \\
56 & $\mathrm{PE}(38: 5 \mathrm{e})$ & -0.045 & -0.043 & 0.061 \\
57 & $\mathrm{PE}(\mathrm{p} 16: 0 / 18: 1)$ & 0.525 & 0.266 & 0.031 \\
58 & $\mathrm{PE}(\mathrm{p} 16: 0 / 18: 2)$ & 0.487 & 0.230 & 0.026 \\
59 & $\mathrm{PE}(\mathrm{P}-16: 0 / 20: 4)$ & 0.011 & 0.018 & 0.071 \\
60 & $\mathrm{PE}(\mathrm{P}-16: 0 / 22: 6)$ & -0.032 & 0.032 & 0.035 \\
61 & $\mathrm{PI}(40: 6)$ & -0.047 & 0.000 & 0.140 \\
62 & $\mathrm{SM}(\mathrm{d} 18: 1 / 16: 0)$ & 0.129 & 0.115 & 0.237 \\
63 & $\mathrm{PE}(18: 3 / 14: 1)$ & 0.624 & 0.413 & 0.003 \\
64 & $\mathrm{PE}(18: 2 / 16: 1)$ & -0.056 & 0.001 & -0.074 \\
65 & $\mathrm{PE}(22: 5 / 18: 0)$ & 0.049 & 0.076 & 0.131 \\
\hline
\end{tabular}

c) Adipose tissue positive electrospray ionization mode (ESI+)

\begin{tabular}{|c|c|c|c|c|c|c|}
\hline$\overline{\text { VAR }}$ & Species & PL1 & PL2 & PL3 & PL4 & PL5 \\
\hline$\overline{1}$ & ChoE(14:0) & 0.152 & 0.004 & 0.623 & 0.330 & -0.829 \\
\hline 2 & ChoE(18:0)fragm & 1.250 & 0.622 & 0.085 & -0.125 & 0.046 \\
\hline 3 & ChoE(18:0)fragm & 0.503 & 0.177 & -0.602 & 0.986 & 0.788 \\
\hline 4 & LysoPC(16:0) & -1.418 & -0.572 & -0.601 & -0.601 & 0.361 \\
\hline 5 & LysoPC(18:0) & -0.820 & -0.282 & 0.805 & 0.296 & -0.122 \\
\hline 6 & $\mathrm{PC}(32: 0)$ & 0.958 & 0.209 & 1.083 & -0.292 & -0.873 \\
\hline 7 & $\mathrm{PC}(32: 1)$ & 1.247 & -1.561 & -1.783 & -0.154 & 0.494 \\
\hline 8 & $\mathrm{PC}(34: 1)$ & 0.113 & 0.964 & 0.285 & 0.484 & -0.313 \\
\hline 9 & $\mathrm{PC}(34: 2)$ & -3.166 & -0.576 & -0.765 & 0.874 & -1.137 \\
\hline 10 & $\mathrm{PC}(36: 2)$ & 1.168 & 0.289 & 0.367 & -1.097 & -0.511 \\
\hline 11 & $\mathrm{PC}(38: 3)$ & 1.179 & -0.417 & 0.285 & -0.469 & 0.137 \\
\hline 12 & $\mathrm{PC}(38: 4)$ & -1.010 & -1.265 & -0.175 & 0.759 & 0.058 \\
\hline 13 & $\mathrm{SM}(\mathrm{d} 18: 1 / 16: 0)$ & -0.677 & 2.407 & 0.167 & 0.081 & 0.050 \\
\hline 14 & $\mathrm{SM}(\mathrm{d} 18: 1 / 24: 0)$ & -1.712 & -0.070 & 1.002 & 0.369 & -0.114 \\
\hline 15 & $\mathrm{SM}(\mathrm{d} 18: 1 / 24: 1)$ & 1.224 & -0.052 & -0.052 & -1.252 & 1.425 \\
\hline \multirow[t]{2}{*}{16} & TG(14:0/16:0/17:0)+ & 1.453 & 1.225 & -0.606 & 0.303 & 0.160 \\
\hline & TG(14:0/18:0/15:0 $)^{\mathrm{a}}$ & & & & & \\
\hline \multirow[t]{2}{*}{17} & TG(14:0/16:0/17:0)+ & 0.096 & 1.463 & -0.670 & 0.638 & 0.535 \\
\hline & $\mathrm{TG}(14: 0 / 18: 0 / 15: 0)^{\mathrm{a}}$ & & & & & \\
\hline 18 & TG(17:0/18:1/18:1) & -1.917 & 0.111 & -0.711 & 0.737 & 0.423 \\
\hline 19 & TG(17:1/18:1/18:2) & 1.109 & 0.204 & 0.588 & -0.377 & -0.669 \\
\hline 20 & TG(18:1/18:2/18:1) & 0.193 & -0.503 & -0.412 & 0.262 & -0.483 \\
\hline 21 & TG(44:1) & 0.339 & -0.611 & 0.282 & -0.707 & 0.494 \\
\hline 22 & TG(45:0) & -0.767 & 1.079 & -1.934 & 0.496 & -0.380 \\
\hline 23 & TG(45:1) & -0.994 & -0.170 & -1.673 & -0.044 & 0.026 \\
\hline 24 & TG(46:1) & -0.451 & -0.152 & -0.153 & -0.025 & 0.511 \\
\hline 25 & TG(46:3) & -0.169 & 0.434 & -0.224 & 0.154 & 0.732 \\
\hline 26 & TG(47:1) & -1.075 & 0.023 & -0.431 & 0.342 & -0.614 \\
\hline 27 & TG(47:2) & -0.647 & 0.252 & 0.250 & -0.030 & -0.907 \\
\hline 28 & TG(47:3) & 1.623 & 0.339 & 1.701 & -1.419 & 0.468 \\
\hline 29 & TG(48:1) & -0.605 & 0.836 & 0.647 & 0.604 & 0.015 \\
\hline 30 & TG(48:2) & -0.458 & 0.088 & -0.339 & 0.476 & -0.149 \\
\hline 31 & TG(48:4) & 1.209 & -0.650 & 1.091 & -1.036 & -0.262 \\
\hline 32 & TG(49:0) & 0.623 & -1.215 & 1.912 & -0.637 & -0.892 \\
\hline 33 & TG(49:2) & -0.527 & -0.189 & 0.438 & 0.232 & -1.411 \\
\hline 34 & TG(50:4) & 1.224 & -0.860 & 0.422 & -0.648 & -0.582 \\
\hline 35 & TG(50:5) & -0.811 & -0.658 & 2.293 & -0.752 & 0.304 \\
\hline 36 & $\mathrm{TG}(51: 2)$ & -0.761 & 0.151 & -0.377 & 0.758 & -0.536 \\
\hline 37 & $\mathrm{TG}(51: 2)$ & 1.041 & -0.050 & -0.933 & 0.114 & 1.621 \\
\hline 38 & TG(51:4) & 1.350 & -0.079 & -0.275 & -0.582 & -0.047 \\
\hline
\end{tabular}




\begin{tabular}{rrrrrrr}
39 & TG(52:2) & 0.690 & -0.083 & 0.119 & 0.091 & 0.364 \\
40 & TG(52:3) & 0.642 & -0.618 & -0.375 & 0.160 & -0.488 \\
41 & TG(52:4) & 1.544 & -0.757 & 0.016 & -0.471 & -0.333 \\
42 & TG(53:2) & -1.956 & 0.271 & 0.943 & 0.873 & -1.266 \\
43 & TG(53:2) & -0.329 & 0.955 & -0.758 & 1.137 & 0.011 \\
44 & TG(53:3) & 0.927 & 0.126 & -0.995 & -0.293 & 0.409 \\
45 & TG(53:5) & -0.714 & 0.689 & -0.619 & 0.841 & 1.342 \\
46 & TG(54:0) & 0.584 & -1.519 & 1.653 & -1.479 & 1.497 \\
47 & TG(54:5) & -1.461 & -0.618 & -0.558 & 0.858 & -0.657 \\
48 & TG(54:6) & -0.369 & 0.906 & -1.346 & 0.908 & -0.452 \\
49 & TG(55:4) & 0.318 & 0.115 & -0.032 & 0.086 & -0.535 \\
50 & TG(55:5) & 0.250 & -1.474 & 1.568 & -0.469 & -0.353 \\
51 & TG(56:4) & 0.214 & -0.425 & -0.926 & 0.220 & 1.479 \\
52 & TG(56:5) & -1.873 & -0.105 & 0.425 & -0.032 & 1.089 \\
53 & TG(56:5) & 3.010 & 0.729 & 0.104 & -0.506 & 0.565 \\
54 & TG(56:6) & 0.679 & 2.207 & -0.142 & 0.352 & -0.844 \\
55 & TG(58:7) & -2.002 & -0.405 & -0.642 & 0.118 & 0.974 \\
\hline
\end{tabular}

d) Adipose tissue negative electrospray ionization mode (ESI-)

\begin{tabular}{|c|c|c|c|c|c|c|}
\hline VAR & Species & PL1 & PL2 & PL3 & PL4 & PL5 \\
\hline 1 & $\operatorname{Cer}(\mathrm{d} 17: 1 / 22: 0)$ & 0.563 & 1.066 & -0.130 & -0.285 & 0.248 \\
\hline 2 & Cer(d18:1/23:1) & 0.540 & 0.043 & -0.096 & -0.095 & -0.369 \\
\hline 3 & $\operatorname{Cer}(\mathrm{d} 18: 1 / 16: 0)$ & 1.966 & -0.926 & -0.601 & 0.100 & 0.354 \\
\hline 4 & Cer(d18:1/18:0) & 1.146 & -0.989 & -0.710 & 0.250 & 0.378 \\
\hline 5 & Cer(d18:1/20:0) & -0.896 & 1.090 & 0.335 & 0.481 & -0.475 \\
\hline 6 & Cer(d18:1/22:0) & 2.735 & -0.970 & -1.148 & 0.442 & 0.415 \\
\hline 7 & Cer(d18:1/23:0) & 0.840 & -0.096 & -0.551 & 0.624 & 0.196 \\
\hline 8 & Cer(d18:1/24:0) & -0.979 & 0.164 & 0.711 & 0.452 & 0.175 \\
\hline 9 & Cer(d18:1/24:1) & 0.108 & 0.386 & -0.632 & -0.640 & 0.159 \\
\hline 10 & Cer(d18:1/25:0) & 0.808 & -0.665 & 0.825 & -0.295 & 1.100 \\
\hline 11 & $\operatorname{Cer}(\mathrm{d} 18: 2 / 16: 0)$ & -2.019 & 0.539 & 0.274 & -0.152 & -0.798 \\
\hline 12 & $\operatorname{Cer}(\mathrm{d} 18: 2 / 18: 0)$ & -2.260 & -0.057 & 0.827 & -0.710 & -0.194 \\
\hline 13 & Cer(d18:1/22:1) & -0.616 & 0.814 & 0.564 & -0.080 & -0.362 \\
\hline 14 & Cer(d18:2/24:1) & -1.693 & 0.448 & -0.324 & -0.554 & -0.101 \\
\hline 15 & Cer(d18:2/25:0) & -0.228 & 0.052 & 0.461 & -0.666 & -0.122 \\
\hline 16 & HexCer(d18:1/22:0) & -0.474 & 0.250 & 0.010 & 0.419 & 0.740 \\
\hline 17 & $\operatorname{PE}(18: 0 / 16: 1)$ & 1.290 & 0.063 & -0.320 & 0.997 & 0.857 \\
\hline 18 & $\operatorname{PE}(18: 0 / 18: 2)$ & -1.062 & 0.973 & 1.076 & 0.358 & 0.261 \\
\hline 19 & PC(18:1/18:0) & 1.292 & -0.427 & -0.213 & 0.155 & 0.427 \\
\hline 20 & $\mathrm{PC}(36: 2)$ & 0.340 & -0.473 & 0.149 & 0.879 & -0.280 \\
\hline 21 & $\mathrm{PC}(36: 3)$ & 0.687 & 0.206 & 0.573 & 0.288 & 0.151 \\
\hline 22 & $\mathrm{PC}(38: 3)$ & 0.009 & -0.342 & -0.447 & -0.315 & 0.098 \\
\hline \multirow[t]{2}{*}{23} & $\mathrm{PE}(16: 0 / 20: 4)+$ & 0.619 & 1.015 & 0.148 & -0.314 & 0.651 \\
\hline & $\operatorname{PE}(18: 2 / 18: 2)^{\mathrm{a}}$ & & & & & \\
\hline 24 & $\mathrm{PE}(18: 0 / 18: 1)$ & 0.576 & -0.159 & -0.004 & -0.619 & 0.587 \\
\hline 25 & $\operatorname{PE}(18: 0 / 20: 3)$ & -0.051 & -0.865 & 0.288 & 0.320 & -0.871 \\
\hline 26 & $\mathrm{PE}(18: 0 / 22: 6)$ & -2.515 & -0.018 & 0.839 & -0.340 & -0.862 \\
\hline 27 & $\operatorname{PE}(18: 1 / 18: 0)$ & -0.161 & 0.132 & -0.964 & 0.281 & -1.130 \\
\hline 28 & $\operatorname{PE}(34: 1)$ & 0.018 & 0.348 & 0.202 & -0.066 & 0.144 \\
\hline 29 & $\operatorname{PE}(36: 2)$ & -1.127 & 0.851 & 0.999 & 0.071 & 0.173 \\
\hline 30 & $\mathrm{PE}(36: 2)$ & 0.263 & -0.200 & -0.095 & 0.271 & -1.328 \\
\hline 31 & $\operatorname{PE}(36: 2 e)$ & -0.455 & 0.622 & 0.280 & -0.790 & 0.244 \\
\hline 32 & $\mathrm{PE}(36: 3)$ & 1.228 & -0.937 & -1.088 & -0.469 & 0.471 \\
\hline 33 & $\operatorname{PE}(36: 3 e)$ & 2.028 & -1.605 & -0.485 & 0.548 & 0.780 \\
\hline 34 & $\mathrm{PE}(36: 5 \mathrm{e})$ & -0.570 & -0.419 & 0.519 & -0.917 & -0.080 \\
\hline
\end{tabular}




\begin{tabular}{llrrrrr}
35 & $\mathrm{PE}(36: 5 \mathrm{e})$ & 0.255 & 0.023 & 0.474 & 0.777 & 0.030 \\
36 & $\mathrm{PE}(38: 4)$ & -0.275 & 0.522 & -0.212 & 0.802 & 0.621 \\
37 & $\mathrm{PE}(38: 4)$ & 1.474 & -0.784 & 0.117 & -0.285 & 0.089 \\
38 & $\mathrm{PE}(38: 4)$ & 1.439 & -0.404 & -0.443 & 0.782 & -0.145 \\
39 & $\mathrm{PE}(38: 4)$ & 0.230 & -0.800 & -0.115 & -0.536 & 0.397 \\
40 & $\mathrm{PE}(38: 4 \mathrm{e})$ & -0.829 & 0.700 & -0.414 & -0.607 & -0.455 \\
41 & $\mathrm{PE}(38: 5 \mathrm{e})$ & 0.039 & 0.483 & -0.285 & -0.020 & 0.469 \\
42 & $\mathrm{PE}(38: 5 \mathrm{e})$ & 0.601 & -0.038 & -0.812 & 0.100 & -0.099 \\
43 & $\mathrm{PE}(\mathrm{p} 16: 0 / 18: 1)$ & -0.208 & 0.286 & 0.811 & -0.156 & -0.355 \\
44 & $\mathrm{PE}(\mathrm{p} 16: 0 / 18: 2)$ & -0.993 & -0.417 & 0.146 & 0.425 & -0.001 \\
45 & $\mathrm{PI}(18: 0 / 20: 4)$ & -1.280 & 1.146 & -0.067 & -0.696 & 0.789 \\
46 & $\mathrm{PI}(36: 3)$ & -0.289 & 0.025 & 0.838 & 0.040 & -0.918 \\
47 & $\mathrm{PI}(36: 4)$ & -0.222 & 0.129 & 0.270 & -0.011 & 0.069 \\
48 & $\mathrm{PI}(38: 3)$ & 0.669 & 0.076 & -0.843 & 0.756 & -0.199 \\
49 & $\mathrm{PI}(38: 5)$ & 1.439 & -1.297 & -0.539 & 0.515 & 0.446 \\
50 & $\mathrm{PI}(40: 5)$ & 0.048 & 0.780 & 0.584 & -0.496 & -0.658 \\
51 & $\mathrm{SM}(\mathrm{d} 18: 1 / 16: 0)$ & 0.738 & -0.111 & -0.249 & -0.886 & 0.164 \\
52 & $\mathrm{PE}(18: 0 / 20: 4)$ & -0.429 & 0.102 & -0.526 & 0.021 & -0.307 \\
53 & $\mathrm{PE}(18: 0 / 20: 0)$ & -1.506 & -0.197 & 0.005 & 0.426 & -0.641 \\
\hline
\end{tabular}

${ }^{\mathrm{a}}$ The ambiguous subspecies indicates that the lipidomic profiling gave two possible identifications of the lipid. ${ }^{\mathrm{b}}$ Abbreviations: Cer, ceramide. HexCer, hexosylceramide. SM, sphingomyelin. TG, triacylglycerol. DG, diacylglycerol. ChoE, cholesteryl ester. LysoPC, lysophophosphatidylcholine. LysoPE, lysophosphatidylethanolamine. PC, phosphatidylcholine. PE, phosphatidylethanolamine. PI, phosphatidylinositol. 


\section{Online Resource Table S3}

Results from the analysis of variance between the control group (CON) and the high-energy feeding group (HIGH) within time points in liver positive electrospray ionization mode (ESI+) dataset. $P$-values were obtained from the analysis performed using MIXED procedure in SAS, with diet as the fixed effect and pair as the random effect. Adjusted- $p$ values were obtained from $p$-values after false discovery rate control.

\begin{tabular}{|c|c|c|c|c|c|c|c|c|}
\hline \multirow[b]{2}{*}{ Lipid subspecies } & \multicolumn{4}{|c|}{$-8 \mathrm{~d}$} & \multicolumn{4}{|c|}{$9 \mathrm{~d}$} \\
\hline & $\mathrm{CON}$ & HIGH & $p$-value & Adjusted- $p$ & $\mathrm{CON}$ & HIGH & $p$-value & Adjusted- $p$ \\
\hline Cer(d18:1/16:0) & -4.691 & -4.375 & 0.1182 & 0.9106 & -4.834 & -4.671 & 0.4952 & 0.9916 \\
\hline Cer(d18:1/18:0) & -7.015 & -6.713 & 0.1157 & 0.6487 & -7.416 & -6.870 & 0.0447 & 0.5220 \\
\hline Cer(d18:1/24:0) & -3.208 & -2.755 & 0.0311 & 0.7513 & -3.125 & -2.776 & 0.1694 & 0.8405 \\
\hline Cer(d18:1/24:1) & -6.086 & -5.474 & 0.0314 & 0.7983 & -6.020 & -5.826 & 0.3005 & 0.9042 \\
\hline $\operatorname{Cer}(\mathrm{d} 18: 1 / 26: 1)$ & -7.265 & -7.318 & 0.9071 & 0.5996 & -7.596 & -7.103 & 0.2653 & 0.8968 \\
\hline Cer(d18:2/23:0) & -5.894 & -5.377 & 0.0349 & 0.9845 & -6.284 & -6.089 & 0.2794 & 0.8988 \\
\hline $\operatorname{DG}(33: 5)$ & -7.883 & -7.896 & 0.9264 & 0.8789 & -7.434 & -7.407 & 0.8579 & 0.9929 \\
\hline DG(34:1) & -9.806 & -9.959 & 0.7641 & 0.9228 & -7.439 & -7.375 & 0.8926 & 0.9929 \\
\hline LysoPC(16:0) & -5.827 & -5.552 & 0.0854 & 0.7015 & -5.851 & -5.539 & 0.0228 & 0.4306 \\
\hline LysoPC(18:0) & -6.143 & -5.305 & 0.0077 & 0.9845 & -5.827 & -5.473 & 0.0943 & 0.7775 \\
\hline LysoPC(18:2) & -8.220 & -8.138 & 0.7955 & 0.8179 & -7.846 & -7.052 & 0.0901 & 0.7648 \\
\hline LysoPE(18:0) & -7.804 & -7.756 & 0.7024 & 0.7582 & -7.905 & -7.732 & 0.2072 & 0.8405 \\
\hline $\mathrm{PC}(16: 0 / 20: 4)$ & 0.681 & 0.792 & 0.1723 & 0.4870 & 0.552 & 0.666 & 0.4574 & 0.9702 \\
\hline $\mathrm{PC}(16: 0 / 20: 5)$ & -0.018 & 0.309 & 0.1656 & 0.4870 & 0.092 & 0.229 & 0.5984 & 0.9929 \\
\hline $\mathrm{PC}(16: 0 / 22: 5)$ & 1.122 & 1.421 & 0.2044 & 0.7341 & 0.795 & 0.992 & 0.5351 & 0.9929 \\
\hline $\mathrm{PC}(16: 1 / 20: 4)$ & -5.566 & -4.863 & 0.0008 & 0.6078 & -4.147 & -4.005 & 0.6935 & 0.9929 \\
\hline $\mathrm{PC}(18: 0 / 22: 6)$ & -0.049 & 0.325 & 0.0181 & 0.4870 & -0.743 & -0.737 & 0.9849 & 0.9957 \\
\hline $\mathrm{PC}(18: 1 / 20: 4)$ & 1.392 & 1.904 & 0.0154 & 0.4870 & 1.054 & 1.211 & 0.6451 & 0.9929 \\
\hline $\mathrm{PC}(30: 0)$ & -6.131 & -5.171 & 0.0795 & 0.4870 & -6.856 & -6.446 & 0.1909 & 0.8405 \\
\hline $\mathrm{PC}(30: 1)$ & -9.482 & -8.359 & 0.0483 & 0.4870 & -9.476 & -8.841 & 0.0809 & 0.7532 \\
\hline $\mathrm{PC}(32: 0)$ & -4.338 & -4.026 & 0.1667 & 0.5128 & -4.432 & -4.411 & 0.8795 & 0.9929 \\
\hline $\mathrm{PC}(32: 1)$ & -3.377 & -2.976 & 0.0204 & 0.4870 & -2.698 & -2.315 & 0.0202 & 0.8968 \\
\hline $\mathrm{PC}(32: 1)$ & -6.593 & -5.485 & 0.2199 & 0.4870 & -6.438 & -6.110 & 0.2270 & 0.4306 \\
\hline $\mathrm{PC}(32: 1 \mathrm{e})$ & -8.011 & -7.619 & 0.0852 & 0.4870 & -8.170 & -8.168 & 0.9822 & 0.9957 \\
\hline $\mathrm{PC}(32: 2)$ & -7.227 & -6.262 & 0.0162 & 0.4870 & -7.146 & -6.663 & 0.0319 & 0.8968 \\
\hline $\mathrm{PC}(32: 2)$ & -9.428 & -8.178 & 0.0228 & 0.4870 & -10.674 & -9.933 & 0.2517 & 0.9284 \\
\hline $\mathrm{PC}(32: 2)$ & -5.959 & -5.123 & 0.1087 & 0.4870 & -5.415 & -4.841 & 0.3345 & 0.4306 \\
\hline $\mathrm{PC}(32: 2 \mathrm{e})$ & -7.343 & -6.366 & 0.0207 & 0.4870 & -6.626 & -6.114 & 0.0114 & 0.4306 \\
\hline $\mathrm{PC}(33: 2)$ & -6.182 & -5.580 & 0.0669 & 0.4870 & -6.199 & -5.869 & 0.3422 & 0.9293 \\
\hline $\mathrm{PC}(34: 1)$ & -0.215 & -0.094 & 0.6272 & 0.4870 & 0.345 & 0.537 & 0.3745 & 0.9628 \\
\hline $\mathrm{PC}(34: 1 \mathrm{e})$ & -7.669 & -7.383 & 0.1087 & 0.4870 & -6.910 & -6.917 & 0.9351 & 0.9957 \\
\hline $\mathrm{PC}(34: 2)$ & -1.296 & -1.124 & 0.0515 & 0.5455 & -0.627 & -0.446 & 0.0315 & 0.9929 \\
\hline $\mathrm{PC}(34: 2)$ & -8.731 & -7.876 & 0.2738 & 0.4900 & -8.295 & -7.795 & 0.5100 & 0.4306 \\
\hline $\mathrm{PC}(34: 3)$ & -3.258 & -2.468 & 0.0083 & 0.4870 & -2.623 & -2.143 & 0.1862 & 0.8405 \\
\hline $\mathrm{PC}(34: 3)$ & -4.289 & -3.996 & 0.3054 & 0.4870 & -3.670 & -3.346 & 0.2062 & 0.8405 \\
\hline $\mathrm{PC}(34: 4)$ & -6.747 & -6.193 & 0.1242 & 0.4870 & -5.848 & -5.321 & 0.1939 & 0.8405 \\
\hline $\mathrm{PC}(34: 4)+\mathrm{PE}(37: 4)^{\mathrm{a}}$ & -7.350 & -7.107 & 0.3745 & 0.4870 & -6.916 & -6.756 & 0.3018 & 0.9042 \\
\hline $\mathrm{PC}(34: 4 \mathrm{e})$ & -8.425 & -8.364 & 0.5441 & 0.4870 & -7.904 & -7.914 & 0.9186 & 0.9957 \\
\hline $\mathrm{PC}(34: 5)$ & -8.319 & -7.351 & 0.0063 & 0.4870 & -9.465 & -9.107 & 0.4418 & 0.9702 \\
\hline $\mathrm{PC}(35: 2)$ & -4.698 & -4.145 & 0.0898 & 0.4870 & -4.359 & -4.159 & 0.2748 & 0.8968 \\
\hline $\mathrm{PC}(36: 1)$ & 0.243 & 0.630 & 0.1733 & 0.4870 & 0.577 & 0.859 & 0.0521 & 0.5729 \\
\hline $\mathrm{PC}(36: 2)$ & -3.765 & -3.345 & 0.1684 & 0.4870 & -3.550 & -3.184 & 0.0175 & 0.4306 \\
\hline $\mathrm{PC}(36: 2)$ & -0.165 & 0.058 & 0.3541 & 0.4870 & 0.465 & 0.682 & 0.0812 & 0.7532 \\
\hline $\mathrm{PC}(36: 3)$ & -1.045 & -0.585 & 0.0583 & 0.4870 & -0.743 & -0.521 & 0.1896 & 0.8405 \\
\hline $\mathrm{PC}(36: 4)$ & -2.655 & -1.880 & 0.0593 & 0.4870 & -3.012 & -3.018 & 0.9906 & 0.9957 \\
\hline $\mathrm{PC}(36: 4 \mathrm{e})$ & -7.319 & -7.392 & 0.6636 & 0.4870 & -7.165 & -6.986 & 0.0299 & 0.4306 \\
\hline $\mathrm{PC}(36: 5)$ & -7.697 & -7.120 & 0.0655 & 0.4870 & -7.918 & -7.770 & 0.6020 & 0.9929 \\
\hline
\end{tabular}




\begin{tabular}{|c|c|c|c|c|c|c|c|c|}
\hline $\mathrm{PC}(36: 6)$ & -5.085 & -4.194 & 0.0132 & 0.4870 & -4.969 & -4.575 & 0.0593 & 0.6294 \\
\hline $\mathrm{PC}(36: 6 e)$ & -6.311 & -5.945 & 0.3500 & 0.4870 & -5.889 & -5.587 & 0.2661 & 0.8968 \\
\hline $\mathrm{PC}(37: 1)$ & -3.503 & -2.155 & 0.0969 & 0.4900 & -4.357 & -4.061 & 0.2721 & 0.8988 \\
\hline $\operatorname{PC}(37: 2)$ & -3.190 & -2.567 & 0.0359 & 0.5815 & -3.473 & -3.508 & 0.4717 & 0.9929 \\
\hline $\mathrm{PC}(37: 5)$ & -3.892 & -3.162 & 0.5145 & 0.4870 & -4.263 & -4.007 & 0.8912 & 0.9628 \\
\hline $\mathrm{PC}(38: 1)$ & -6.705 & -6.359 & 0.7211 & 0.4870 & -7.047 & -6.975 & 0.3243 & 0.9929 \\
\hline $\mathrm{PC}(38: 1)$ & -4.429 & -3.536 & 0.0668 & 0.4870 & -5.035 & -4.565 & 0.7466 & 0.8968 \\
\hline $\mathrm{PC}(38: 1)$ & -7.226 & -5.919 & 0.0719 & 0.4870 & -8.056 & -7.896 & 0.6115 & 0.9867 \\
\hline $\operatorname{PC}(38: 3)$ & -3.275 & -2.716 & 0.4851 & 0.4870 & -4.343 & -4.193 & 0.4162 & 0.9929 \\
\hline $\mathrm{PC}(38: 3)$ & -0.547 & -0.173 & 0.0371 & 0.4870 & -0.938 & -1.097 & 0.9505 & 0.9929 \\
\hline $\operatorname{PC}(38: 3)$ & -3.744 & -3.651 & 0.0319 & 0.4870 & -4.345 & -4.628 & 0.0286 & 0.9172 \\
\hline PC(38:4) & -0.647 & -0.091 & 0.0794 & 0.4870 & -1.616 & -1.590 & 0.1486 & 0.9957 \\
\hline $\mathrm{PC}(38: 4)$ & -0.954 & -0.839 & 0.0794 & 0.6959 & -1.063 & -0.939 & 0.6488 & 0.9649 \\
\hline $\mathrm{PC}(38: 4 \mathrm{e})$ & -5.887 & -4.967 & 0.2742 & 0.4870 & -6.609 & -6.280 & 0.7930 & 0.4306 \\
\hline $\mathrm{PC}(38: 5 \mathrm{e})$ & -7.631 & -7.476 & 0.1428 & 0.4870 & -6.884 & -6.942 & 0.3521 & 0.9929 \\
\hline $\mathrm{PC}(38: 5 \mathrm{e})$ & -4.167 & -3.640 & 0.0677 & 0.4870 & -4.157 & -4.058 & 0.6561 & 0.9929 \\
\hline $\mathrm{PC}(38: 5 \mathrm{e})$ & -8.410 & -7.629 & 0.0427 & 0.6865 & -8.687 & -8.325 & 0.1804 & 0.8405 \\
\hline PC(38:6) & -4.445 & -4.004 & 0.2748 & 0.4870 & -3.971 & -3.769 & 0.2020 & 0.9293 \\
\hline $\mathrm{PC}(38: 6)$ & -3.440 & -2.989 & 0.1756 & 0.6056 & -3.404 & -3.280 & 0.9801 & 0.9929 \\
\hline PC(38:6e) & -5.460 & -4.695 & 0.1776 & 0.5775 & -5.744 & -5.524 & 0.6994 & 0.8405 \\
\hline $\mathrm{PC}(38: 7 e)$ & -6.845 & -6.531 & 0.0277 & 0.8754 & -7.193 & -6.912 & 0.0847 & 0.8405 \\
\hline PC(39:6) & -6.946 & -6.895 & 0.1059 & 0.5666 & -7.125 & -6.853 & 0.3057 & 0.8405 \\
\hline $\mathrm{PC}(40: 3)$ & -7.769 & -7.322 & 0.1736 & 0.4870 & -9.107 & -9.115 & 0.7267 & 0.9957 \\
\hline $\mathrm{PC}(40: 3 \mathrm{e})$ & -6.867 & -6.637 & 0.0568 & 0.9724 & -6.645 & -6.681 & 0.4218 & 0.9929 \\
\hline $\mathrm{PC}(40: 4)$ & -6.096 & -5.499 & 0.1810 & 0.8078 & -6.086 & -5.818 & 0.8771 & 0.9042 \\
\hline PC(40:4) & -3.412 & -2.563 & 0.1315 & 0.8549 & -4.579 & -4.110 & 0.0232 & 0.7626 \\
\hline $\mathrm{PC}(40: 4 \mathrm{e})$ & -7.182 & -6.898 & 0.4365 & 0.9604 & -7.131 & -7.106 & 0.8813 & 0.9929 \\
\hline $\mathrm{PC}(40: 5)$ & -0.269 & 0.323 & 0.0030 & 0.4870 & -0.630 & -0.405 & 0.7213 & 0.9649 \\
\hline PC(40:5e) & -8.027 & -7.679 & 0.1867 & 0.4870 & -7.878 & -7.941 & 0.3062 & 0.9929 \\
\hline $\mathrm{PC}(40: 7)$ & -7.734 & -7.250 & 0.2648 & 0.4870 & -7.910 & -7.488 & 0.4131 & 0.4306 \\
\hline $\mathrm{PC}(40: 7)$ & -5.797 & -5.598 & 0.0130 & 0.8516 & -5.851 & -5.811 & 0.0353 & 0.9929 \\
\hline PC(40:8) & -7.426 & -8.020 & 0.0213 & 0.8233 & -7.474 & -7.384 & 0.0120 & 0.9929 \\
\hline PC(40:8e) & -8.174 & -7.627 & 0.7495 & 0.6752 & -8.397 & -8.194 & 0.4442 & 0.9042 \\
\hline PC(p16:0/20:4) & -0.709 & -0.420 & 0.2060 & 0.6078 & -0.534 & -0.313 & 0.6877 & 0.9649 \\
\hline $\operatorname{PE}(32: 1)$ & -7.229 & -6.271 & 0.0150 & 0.9776 & -7.041 & -6.523 & 0.0270 & 0.4563 \\
\hline $\operatorname{PE}(32: 3 \mathrm{e})$ & -7.788 & -6.954 & 0.0103 & 0.9724 & -6.660 & -6.213 & 0.0090 & 0.4306 \\
\hline $\operatorname{PE}(34: 1)$ & -4.639 & -4.513 & 0.0179 & 0.4910 & -3.506 & -3.301 & 0.0285 & 0.9702 \\
\hline $\operatorname{PE}(34: 2)$ & -5.549 & -5.240 & 0.0963 & 0.8864 & -4.219 & -4.106 & 0.3472 & 0.9929 \\
\hline $\operatorname{PE}(34: 2 \mathrm{e})$ & -7.989 & -6.732 & 0.7965 & 0.4870 & -7.527 & -6.658 & 0.9628 & 0.4306 \\
\hline $\operatorname{PE}(34: 3 e)$ & -7.103 & -6.102 & 0.1529 & 0.4900 & -6.049 & -5.599 & 0.1958 & 0.4306 \\
\hline $\operatorname{PE}(34: 4 e)$ & -8.533 & -7.479 & 0.0812 & 0.4870 & -8.246 & -7.856 & 0.2536 & 0.4306 \\
\hline $\operatorname{PE}(36: 0)$ & -5.459 & -4.787 & 0.3903 & 0.4870 & -6.298 & -6.039 & 0.3157 & 0.9293 \\
\hline $\operatorname{PE}(36: 0)$ & -8.167 & -8.122 & 0.1311 & 0.8226 & -8.103 & -8.098 & 0.2709 & 0.9957 \\
\hline $\operatorname{PE}(36: 1)$ & -5.553 & -5.237 & 0.0653 & 0.8226 & -4.720 & -4.385 & 0.0081 & 0.9103 \\
\hline $\operatorname{PE}(36: 1)$ & -3.928 & -3.451 & 0.0774 & 0.4870 & -3.817 & -3.515 & 0.2715 & 0.8968 \\
\hline $\operatorname{PE}(36: 1)$ & -3.300 & -2.749 & 0.0150 & 0.7935 & -2.751 & -2.573 & 0.9421 & 0.8405 \\
\hline $\operatorname{PE}(36: 2)$ & -2.938 & -2.510 & 0.0350 & 0.4870 & -1.833 & -1.566 & 0.1542 & 0.8968 \\
\hline $\operatorname{PE}(36: 2 \mathrm{e})$ & -8.466 & -9.000 & 0.0265 & 0.5780 & -8.678 & -8.696 & 0.0023 & 0.9957 \\
\hline $\operatorname{PE}(36: 2 \mathrm{e})$ & -8.982 & -8.296 & 0.1612 & 0.7582 & -8.512 & -8.343 & 0.5545 & 0.4306 \\
\hline $\operatorname{PE}(36: 2 e)$ & -8.471 & -7.694 & 0.6513 & 0.5815 & -8.161 & -7.770 & 0.8816 & 0.8968 \\
\hline $\operatorname{PE}(36: 3)$ & -4.935 & -4.358 & 0.0136 & 0.7423 & -4.247 & -3.911 & 0.6665 & 0.8405 \\
\hline $\operatorname{PE}(36: 3 e)$ & -7.043 & -6.590 & 0.5717 & 0.4870 & -6.440 & -6.268 & 0.7771 & 0.9929 \\
\hline $\operatorname{PE}(36: 3 e)$ & -8.780 & -8.019 & 0.1880 & 0.8731 & -8.189 & -7.791 & 0.4089 & 0.4306 \\
\hline $\operatorname{PE}(36: 4)$ & -5.345 & -5.445 & 0.2337 & 0.7983 & -4.395 & -4.352 & 0.4178 & 0.9929 \\
\hline $\mathrm{PE}(36: 4 \mathrm{e})$ & -8.708 & -8.004 & 0.1471 & 0.6078 & -8.857 & -8.749 & 0.2059 & 0.9929 \\
\hline
\end{tabular}




\begin{tabular}{|c|c|c|c|c|c|c|c|c|}
\hline $\operatorname{PE}(36: 5)$ & -6.680 & -6.502 & 0.0756 & 0.8078 & -5.709 & -5.635 & 0.0876 & 0.9929 \\
\hline $\operatorname{PE}(36: 5 \mathrm{e})$ & -8.570 & -8.181 & 0.0705 & 0.4870 & -8.809 & -9.027 & 0.4096 & 0.9649 \\
\hline $\operatorname{PE}(36: 5 e)$ & -6.961 & -6.776 & 0.2331 & 0.6932 & -6.669 & -6.581 & 0.1099 & 0.9649 \\
\hline $\operatorname{PE}(38: 0)$ & -6.722 & -6.070 & 0.0515 & 0.5567 & -7.952 & -7.641 & 0.0218 & 0.8405 \\
\hline $\operatorname{PE}(38: 1)$ & -3.059 & -2.177 & 0.1191 & 0.6865 & -3.271 & -2.854 & 0.3793 & 0.7648 \\
\hline $\operatorname{PE}(38: 1)$ & -6.990 & -6.049 & 0.0283 & 0.5423 & -8.052 & -7.677 & 0.7252 & 0.9649 \\
\hline $\mathrm{PE}(38: 1 \mathrm{e})$ & -8.010 & -7.694 & 0.5932 & 0.6097 & -7.493 & -7.321 & 0.1466 & 0.8369 \\
\hline $\operatorname{PE}(38: 2)$ & -9.163 & -8.297 & 0.1845 & 0.6846 & -8.470 & -8.160 & 0.3393 & 0.4306 \\
\hline $\operatorname{PE}(38: 2)$ & -6.728 & -6.384 & 0.0664 & 0.4910 & -7.027 & -7.111 & 0.7453 & 0.9929 \\
\hline $\operatorname{PE}(38: 2)$ & -7.513 & -7.024 & 0.3954 & 0.9553 & -8.102 & -7.752 & 0.2550 & 0.9628 \\
\hline $\operatorname{PE}(38: 3)$ & -6.928 & -6.790 & 0.0837 & 0.8754 & -7.172 & -7.477 & 0.8755 & 0.8405 \\
\hline $\operatorname{PE}(38: 3)$ & -3.932 & -3.598 & 0.3317 & 0.5943 & -4.320 & -4.431 & 0.3075 & 0.9929 \\
\hline $\operatorname{PE}(38: 3)$ & -7.784 & -7.409 & 0.1115 & 0.8731 & -8.305 & -8.724 & 0.8506 & 0.9293 \\
\hline $\operatorname{PE}(38: 4)$ & -1.819 & -1.649 & 0.5267 & 0.8789 & -1.581 & -1.431 & 0.4554 & 0.8968 \\
\hline $\operatorname{PE}(38: 4)$ & -4.871 & -4.426 & 0.1199 & 0.5280 & -5.677 & -5.741 & 0.6703 & 0.9929 \\
\hline $\operatorname{PE}(38: 5)$ & -7.028 & -6.659 & 0.0349 & 0.4870 & -6.695 & -6.658 & 0.0240 & 0.9929 \\
\hline $\operatorname{PE}(38: 5)$ & -4.672 & -4.398 & 0.3971 & 0.7272 & -4.161 & -4.014 & 0.6096 & 0.9042 \\
\hline $\mathrm{PE}(38: 5 \mathrm{e})$ & -6.122 & -5.990 & 0.0175 & 0.5815 & -6.209 & -6.076 & 0.2815 & 0.9702 \\
\hline $\operatorname{PE}(38: 6)$ & -8.258 & -7.759 & 0.0221 & 0.4870 & -7.623 & -7.489 & 0.8006 & 0.9929 \\
\hline $\operatorname{PE}(38: 6 e)$ & -8.695 & -8.484 & 0.0518 & 0.4870 & -8.341 & -8.301 & 0.9687 & 0.9929 \\
\hline $\operatorname{PE}(38: 6 e)$ & -5.711 & -5.059 & 0.9741 & 0.4870 & -5.595 & -5.257 & 0.9772 & 0.4306 \\
\hline $\operatorname{PE}(40: 3)$ & -7.656 & -7.645 & 0.3559 & 0.4870 & -8.432 & -8.444 & 0.7867 & 0.9957 \\
\hline $\operatorname{PE}(40: 3)$ & -5.797 & -5.074 & 0.6025 & 0.4870 & -6.423 & -6.436 & 0.9631 & 0.9957 \\
\hline $\operatorname{PE}(40: 4)$ & -6.203 & -5.917 & 0.1477 & 0.9776 & -6.747 & -6.818 & 0.1638 & 0.9929 \\
\hline $\operatorname{PE}(40: 4 e)$ & -8.218 & -8.277 & 0.0387 & 0.4870 & -7.855 & -7.848 & 0.3773 & 0.9957 \\
\hline $\operatorname{PE}(40: 5)$ & -8.727 & -8.211 & 0.0922 & 0.4870 & -9.418 & -8.875 & 0.1008 & 0.8405 \\
\hline PE(40:6) & -2.711 & -2.574 & 0.5026 & 0.4870 & -2.510 & -2.452 & 0.7467 & 0.9929 \\
\hline $\operatorname{PE}(40: 6)$ & -3.926 & -3.535 & 0.5483 & 0.8754 & -3.450 & -3.246 & 0.6628 & 0.7878 \\
\hline $\operatorname{PE}(40: 6)$ & -8.136 & -7.923 & 0.5741 & 0.8549 & -7.608 & -7.524 & 0.7954 & 0.9929 \\
\hline $\operatorname{PE}(40: 6)$ & -5.734 & -5.560 & 0.2078 & 0.7513 & -5.662 & -5.595 & 0.9782 & 0.9929 \\
\hline $\operatorname{PE}(40: 6 e)$ & -6.448 & -6.232 & 0.8275 & 0.5525 & -6.638 & -6.642 & 0.1682 & 0.9957 \\
\hline $\mathrm{PE}(\mathrm{p} 16: 0 / 22: 6)$ & -5.106 & -4.566 & 0.0202 & 0.4870 & -5.663 & -5.405 & 0.2651 & 0.8968 \\
\hline PE(p18:0/20:4) & -3.760 & -3.845 & 0.5149 & 0.7198 & -3.799 & -4.120 & 0.0146 & 0.4306 \\
\hline PI(38:5) & -8.166 & -7.618 & 0.0745 & 0.5943 & -7.581 & -7.287 & 0.1984 & 0.8405 \\
\hline $\mathrm{SM}(\mathrm{d} 18: 1 / 14: 0)$ & -6.261 & -5.882 & 0.2271 & 0.7246 & -6.284 & -6.015 & 0.0969 & 0.7775 \\
\hline $\operatorname{SM}(\mathrm{d} 18: 1 / 16: 0)$ & -1.791 & -1.520 & 0.0678 & 0.5282 & -2.104 & -1.910 & 0.2487 & 0.8968 \\
\hline $\mathrm{SM}(\mathrm{d} 18: 1 / 16: 1)$ & -6.975 & -6.594 & 0.0359 & 0.5431 & -7.273 & -7.164 & 0.5265 & 0.9929 \\
\hline $\mathrm{SM}(\mathrm{d} 18: 1 / 18: 0)$ & -4.369 & -4.152 & 0.0921 & 0.4870 & -4.590 & -4.470 & 0.1779 & 0.8405 \\
\hline $\operatorname{SM}(\mathrm{d} 18: 1 / 20: 0)$ & -5.928 & -5.651 & 0.1445 & 0.4870 & -5.702 & -5.562 & 0.3014 & 0.9042 \\
\hline $\operatorname{SM}(\mathrm{d} 18: 1 / 21: 0)$ & -5.398 & -4.994 & 0.1330 & 0.4870 & -5.212 & -4.963 & 0.1685 & 0.8405 \\
\hline $\operatorname{SM}(\mathrm{d} 18: 1 / 23: 0)$ & -2.658 & -2.170 & 0.0070 & 0.6703 & -2.517 & -2.342 & 0.1922 & 0.8405 \\
\hline $\operatorname{SM}(\mathrm{d} 18: 1 / 23: 1)$ & -5.033 & -4.651 & 0.0224 & 0.4900 & -5.384 & -5.168 & 0.0292 & 0.4306 \\
\hline $\operatorname{SM}(\mathrm{d} 18: 1 / 24: 1)$ & -4.051 & -4.199 & 0.1353 & 0.4870 & -4.416 & -4.318 & 0.6293 & 0.9929 \\
\hline $\mathrm{TG}(14: 0 / 16: 0 / 16: 0)+$ & -3.498 & -3.651 & 0.8021 & 0.4870 & -0.639 & -0.444 & 0.6194 & 0.9929 \\
\hline \multicolumn{9}{|l|}{$\mathrm{TG}(16: 0 / 18: 0 / 12: 0)^{\mathrm{a}}$} \\
\hline TG(14:0/16:0/17:0)+ & -5.647 & -5.751 & 0.7767 & 0.4870 & -2.964 & -2.624 & 0.3528 & 0.9293 \\
\hline \multicolumn{9}{|l|}{$\mathrm{TG}(14: 0 / 18: 0 / 15: 0)^{\mathrm{a}}$} \\
\hline TG(14:0/16:0/17:0)+ & -5.124 & -5.201 & 0.8118 & 0.4870 & -2.431 & -2.083 & 0.3536 & 0.9293 \\
\hline \multicolumn{9}{|l|}{$\mathrm{TG}(14: 0 / 18: 0 / 15: 0)^{\mathrm{a}}$} \\
\hline TG(16:0/16:0/16:0)+ & -2.732 & -3.060 & 0.6556 & 0.4870 & 0.079 & 0.215 & 0.8070 & 0.9929 \\
\hline \multicolumn{9}{|l|}{$\operatorname{TG}(14: 0 / 16: 0 / 18: 0)^{\mathrm{a}}$} \\
\hline TG(16:0/18:1/18:1) & -0.638 & -0.688 & 0.9556 & 0.4870 & 1.859 & 1.688 & 0.7769 & 0.9929 \\
\hline TG(16:0/18:1/20:1)+ & -1.955 & -1.976 & 0.9832 & 0.4870 & -0.592 & -0.795 & 0.7717 & 0.9929 \\
\hline $\mathrm{TG}(18: 0 / 18: 1 / 18: 1)^{\mathrm{a}}$ & & & & & & & & \\
\hline
\end{tabular}




\begin{tabular}{|c|c|c|c|c|c|c|c|c|}
\hline \multicolumn{9}{|l|}{$\begin{array}{l}\text { TG(16:0/18:2/18:1)+ } \\
\text { TG(16:1/18:1/18:1) }\end{array}$} \\
\hline $\mathrm{TG}(17: 1 / 18: 1 / 18: 2)$ & -7.380 & -7.303 & 0.8452 & 0.4910 & -4.137 & -4.049 & 0.8766 & 0.9929 \\
\hline TG(18:1/16:1/18:2)+ & -4.114 & -4.539 & 0.5108 & 0.5128 & -0.471 & -0.451 & 0.9756 & 0.9957 \\
\hline \multicolumn{9}{|l|}{$\mathrm{TG}(18: 2 / 18: 2 / 16: 0)^{\mathrm{a}}$} \\
\hline TG(18:1/18:1/18:1) & -2.671 & -2.746 & 0.9250 & 0.8561 & -0.526 & -0.662 & 0.8580 & 0.9929 \\
\hline TG(18:1/18:1/22:1)+ & -10.295 & -10.202 & 0.8279 & 0.5128 & -7.965 & -7.665 & 0.6693 & 0.9929 \\
\hline \multicolumn{9}{|l|}{$\mathrm{TG}(20: 1 / 20: 1 / 18: 1)^{\mathrm{a}}$} \\
\hline $\mathrm{TG}(37: 0)$ & -7.163 & -6.689 & 0.0847 & 0.7666 & -6.994 & -6.831 & 0.4445 & 0.9702 \\
\hline $\mathrm{TG}(38: 0)$ & -8.541 & -8.000 & 0.0603 & 0.4870 & -8.463 & -8.163 & 0.0745 & 0.7375 \\
\hline $\mathrm{TG}(42: 0)$ & -9.176 & -8.812 & 0.5825 & 0.4900 & -7.014 & -6.345 & 0.1935 & 0.8405 \\
\hline $\mathrm{TG}(42: 1)$ & -9.947 & -9.980 & 0.9676 & 0.4870 & -7.481 & -6.846 & 0.2405 & 0.8968 \\
\hline $\mathrm{TG}(44: 0)$ & -6.701 & -6.655 & 0.9417 & 0.4870 & -3.878 & -3.354 & 0.1961 & 0.8405 \\
\hline $\mathrm{TG}(44: 1)$ & -7.135 & -7.159 & 0.9703 & 0.6173 & -3.947 & -3.414 & 0.2488 & 0.8968 \\
\hline $\mathrm{TG}(44: 2)$ & -9.759 & -9.692 & 0.9084 & 0.4870 & -6.579 & -5.940 & 0.3751 & 0.9628 \\
\hline $\mathrm{TG}(45: 0)$ & -7.647 & -7.410 & 0.4650 & 0.4870 & -4.725 & -4.286 & 0.2094 & 0.8405 \\
\hline $\mathrm{TG}(46: 1)$ & -4.718 & -4.988 & 0.5975 & 0.4870 & -1.396 & -1.011 & 0.4566 & 0.9702 \\
\hline TG(46:2) & -6.932 & -6.703 & 0.6904 & 0.4870 & -3.161 & -2.652 & 0.4225 & 0.9649 \\
\hline $\mathrm{TG}(46: 3)$ & -8.671 & -8.329 & 0.0733 & 0.4870 & -8.367 & -8.240 & 0.1917 & 0.8405 \\
\hline TG(46:3) & -9.634 & -9.473 & 0.6123 & 0.4870 & -5.852 & -5.294 & 0.3883 & 0.9649 \\
\hline $\mathrm{TG}(47: 1)$ & -6.377 & -6.408 & 0.9320 & 0.4870 & -2.729 & -2.242 & 0.2565 & 0.8968 \\
\hline $\mathrm{TG}(47: 2)$ & -7.950 & -7.938 & 0.9657 & 0.4870 & -4.188 & -3.852 & 0.5161 & 0.9929 \\
\hline $\mathrm{TG}(47: 3)$ & -10.378 & -10.240 & 0.6066 & 0.4870 & -6.559 & -6.253 & 0.5163 & 0.9929 \\
\hline $\mathrm{TG}(48: 1)$ & -2.804 & -3.048 & 0.7252 & 0.4870 & 0.243 & 0.534 & 0.5232 & 0.9929 \\
\hline TG(48:2) & -4.424 & -4.486 & 0.9116 & 0.4870 & -0.735 & -0.581 & 0.7642 & 0.9929 \\
\hline $\mathrm{TG}(48: 3)$ & -7.020 & -6.757 & 0.3554 & 0.5666 & -2.643 & -2.183 & 0.4221 & 0.9649 \\
\hline TG(48:4) & -8.790 & -8.635 & 0.2294 & 0.8913 & -5.073 & -4.570 & 0.4751 & 0.9868 \\
\hline TG(48:5) & -6.221 & -6.440 & 0.1241 & 0.7174 & -6.340 & -6.374 & 0.7922 & 0.9929 \\
\hline TG(49:0) & -6.388 & -6.390 & 0.9673 & 0.4870 & -4.081 & -3.737 & 0.6883 & 0.9929 \\
\hline TG(49:0) & -6.199 & -6.231 & 0.9980 & 0.4870 & -3.520 & -3.211 & 0.5360 & 0.9929 \\
\hline TG(49:1) & -4.874 & -5.120 & 0.6951 & 0.4870 & -1.130 & -1.045 & 0.8861 & 0.9929 \\
\hline $\mathrm{TG}(49: 2)$ & -6.255 & -6.260 & 0.9888 & 0.4870 & -2.274 & -2.042 & 0.6827 & 0.9929 \\
\hline TG(49:3) & -8.124 & -7.964 & 0.3795 & 0.7513 & -4.291 & -3.836 & 0.3911 & 0.9649 \\
\hline TG(49:4) & -9.849 & -9.717 & 0.5848 & 0.4870 & -6.542 & -6.289 & 0.6209 & 0.9929 \\
\hline $\mathrm{TG}(50: 0)$ & -6.553 & -6.620 & 0.8316 & 0.4870 & -3.990 & -3.654 & 0.4481 & 0.9702 \\
\hline $\mathrm{TG}(50: 0)$ & -3.670 & -3.933 & 0.8139 & 0.4870 & -1.765 & -1.574 & 0.7632 & 0.9929 \\
\hline $\mathrm{TG}(50: 1)$ & -8.658 & -8.532 & 0.6959 & 0.4900 & -5.630 & -5.563 & 0.8314 & 0.9929 \\
\hline $\mathrm{TG}(50: 1)$ & -1.310 & -1.544 & 0.7946 & 0.4870 & 1.539 & 1.646 & 0.8396 & 0.9929 \\
\hline $\mathrm{TG}(50: 2)$ & -6.271 & -6.010 & 0.5042 & 0.4870 & -7.542 & -7.681 & 0.7980 & 0.9929 \\
\hline $\mathrm{TG}(50: 2)$ & -7.289 & -7.538 & 0.5786 & 0.4870 & -3.268 & -3.041 & 0.6693 & 0.9929 \\
\hline $\mathrm{TG}(50: 2)$ & -2.636 & -2.747 & 0.8849 & 0.5423 & 0.701 & 0.903 & 0.7350 & 0.9929 \\
\hline $\mathrm{TG}(50: 3)$ & -4.886 & -5.135 & 0.5686 & 0.4870 & -1.037 & -0.760 & 0.6312 & 0.9929 \\
\hline TG(50:6) & -11.043 & -10.931 & 0.7472 & 0.4870 & -7.686 & -7.394 & 0.6832 & 0.9929 \\
\hline $\mathrm{TG}(51: 1)$ & -6.097 & -5.391 & 0.0901 & 0.6904 & -5.339 & -4.865 & 0.0252 & 0.4306 \\
\hline $\mathrm{TG}(51: 1)$ & -5.037 & -5.212 & 0.8700 & 0.4870 & -2.015 & -1.822 & 0.7990 & 0.9929 \\
\hline $\mathrm{TG}(51: 1)$ & -5.304 & -5.634 & 0.6817 & 0.8343 & -2.156 & -2.153 & 0.9957 & 0.9957 \\
\hline $\mathrm{TG}(51: 2)$ & -4.953 & -4.997 & 0.7535 & 0.4870 & -1.377 & -1.340 & 0.9779 & 0.9957 \\
\hline $\mathrm{TG}(51: 2)$ & -9.246 & -9.528 & 0.9529 & 0.5943 & -5.185 & -5.167 & 0.9590 & 0.9957 \\
\hline $\mathrm{TG}(51: 3)$ & -6.868 & -6.842 & 0.9550 & 0.4870 & -2.775 & -2.720 & 0.9270 & 0.9957 \\
\hline $\mathrm{TG}(51: 4)$ & -8.497 & -8.154 & 0.0851 & 0.4870 & -4.485 & -4.200 & 0.6394 & 0.9929 \\
\hline $\mathrm{TG}(51: 5)$ & -8.946 & -9.061 & 0.5752 & 0.4870 & -8.277 & -8.321 & 0.8053 & 0.9929 \\
\hline $\mathrm{TG}(51: 5)$ & -10.413 & -10.326 & 0.7752 & 0.4870 & -6.858 & -6.631 & 0.7007 & 0.9929 \\
\hline $\mathrm{TG}(52: 0)$ & -4.440 & -4.788 & 0.6287 & 0.4870 & -4.046 & -4.342 & 0.4828 & 0.9929 \\
\hline $\mathrm{TG}(52: 0)$ & -5.473 & -5.647 & 0.7964 & 0.8908 & -2.499 & -2.151 & 0.7220 & 0.9916 \\
\hline
\end{tabular}




\begin{tabular}{|c|c|c|c|c|c|c|c|c|}
\hline $\mathrm{TG}(52: 1)$ & -1.801 & -2.285 & 0.6863 & 0.4870 & 0.188 & 0.197 & 0.9892 & 0.9957 \\
\hline $\mathrm{TG}(52: 3)$ & -7.311 & -7.422 & 0.7429 & 0.4870 & -6.900 & -6.800 & 0.3992 & 0.9649 \\
\hline TG(52:5) & -7.400 & -7.050 & 0.0024 & 0.4870 & -2.984 & -2.898 & 0.9081 & 0.9957 \\
\hline TG(53:0) & -9.331 & -9.662 & 0.4221 & 0.4870 & -7.656 & -7.692 & 0.4515 & 0.9957 \\
\hline TG(53:0) & -8.717 & -8.690 & 0.9222 & 0.4900 & -8.284 & -8.385 & 0.5628 & 0.9929 \\
\hline TG(53:0) & -8.423 & -8.318 & 0.9807 & 0.4870 & -6.336 & -6.017 & 0.8893 & 0.9702 \\
\hline TG(53:0) & -9.119 & -9.247 & 0.8093 & 0.4870 & -8.626 & -9.071 & 0.9589 & 0.9929 \\
\hline TG(53:1) & -6.828 & -6.871 & 0.8434 & 0.4870 & -5.274 & -5.106 & 0.5354 & 0.9929 \\
\hline TG(53:1) & -8.195 & -8.134 & 0.9234 & 0.4870 & -4.776 & -4.363 & 0.8860 & 0.9929 \\
\hline TG(53:1) & -6.925 & -7.047 & 0.9756 & 0.4870 & -5.063 & -5.150 & 0.8200 & 0.9929 \\
\hline TG(53:3) & -10.641 & -10.339 & 0.4587 & 0.4870 & -7.300 & -7.196 & 0.8722 & 0.9929 \\
\hline TG(53:3) & -6.484 & -6.500 & 0.9818 & 0.4870 & -3.486 & -3.636 & 0.8218 & 0.9929 \\
\hline TG(53:5) & -8.452 & -8.308 & 0.6339 & 0.9035 & -7.736 & -8.012 & 0.1310 & 0.8405 \\
\hline TG(53:5) & -9.352 & -9.044 & 0.3270 & 0.4870 & -5.874 & -5.581 & 0.6627 & 0.9929 \\
\hline TG(53:6) & -4.531 & -4.440 & 0.8473 & 0.8149 & -3.116 & -3.302 & 0.6619 & 0.9929 \\
\hline $\mathrm{TG}(54: 0)$ & -6.476 & -6.937 & 0.6552 & 0.4870 & -6.826 & -7.375 & 0.4256 & 0.9929 \\
\hline $\mathrm{TG}(54: 0)$ & -6.599 & -6.465 & 0.7404 & 0.4870 & -4.033 & -3.656 & 0.5624 & 0.9649 \\
\hline TG(54:1) & -3.810 & -4.091 & 0.7060 & 0.6669 & -3.044 & -3.451 & 0.5647 & 0.9929 \\
\hline $\mathrm{TG}(54: 1)$ & -6.086 & -6.256 & 0.8425 & 0.4870 & -2.841 & -2.472 & 0.6378 & 0.9929 \\
\hline $\mathrm{TG}(54: 2)$ & -8.339 & -8.441 & 0.6707 & 0.4870 & -6.704 & -6.876 & 0.6641 & 0.9929 \\
\hline $\mathrm{TG}(54: 2)$ & -5.676 & -5.709 & 0.9711 & 0.4870 & -3.480 & -3.584 & 0.9017 & 0.9957 \\
\hline $\mathrm{TG}(54: 4)$ & -7.731 & -7.812 & 0.6594 & 0.9988 & -4.751 & -4.833 & 0.9073 & 0.9957 \\
\hline TG(54:5) & -6.665 & -6.332 & 0.0538 & 0.6659 & -2.406 & -2.094 & 0.7352 & 0.9929 \\
\hline TG(54:6) & -8.327 & -8.062 & 0.5589 & 0.9944 & -4.258 & -4.009 & 0.7410 & 0.9929 \\
\hline TG(54:9) & -5.358 & -5.494 & 0.8852 & 0.4870 & -3.236 & -3.371 & 0.8399 & 0.9929 \\
\hline TG(55:1) & -9.322 & -9.388 & 0.2419 & 0.4870 & -7.581 & -7.307 & 0.8444 & 0.9929 \\
\hline TG(55:1) & -9.536 & -9.175 & 0.9535 & 0.5423 & -7.228 & -7.114 & 0.7333 & 0.9929 \\
\hline $\mathrm{TG}(55: 2)$ & -10.731 & -10.610 & 0.6958 & 0.4870 & -7.481 & -7.240 & 0.7481 & 0.9929 \\
\hline $\mathrm{TG}(55: 2)$ & -8.303 & -8.234 & 0.9476 & 0.4870 & -6.571 & -6.522 & 0.9344 & 0.9957 \\
\hline TG(55:3) & -8.350 & -8.324 & 0.9650 & 0.9988 & -6.957 & -6.851 & 0.8356 & 0.9929 \\
\hline TG(55:4) & -9.311 & -9.269 & 0.8511 & 0.4870 & -7.552 & -7.432 & 0.8135 & 0.9929 \\
\hline TG(55:5) & -9.858 & -9.486 & 0.0774 & 0.6585 & -6.828 & -6.343 & 0.4879 & 0.9916 \\
\hline TG(55:6) & -10.491 & -10.020 & 0.2217 & 0.7395 & -7.059 & -6.765 & 0.6810 & 0.9929 \\
\hline TG(56:0) & -10.425 & -10.664 & 0.7298 & 0.4870 & -8.557 & -8.423 & 0.8866 & 0.9929 \\
\hline TG(56:1) & -9.593 & -9.640 & 0.8854 & 0.4870 & -8.373 & -8.497 & 0.8385 & 0.9929 \\
\hline TG(56:1) & -9.064 & -9.222 & 0.9479 & 0.4870 & -6.688 & -6.717 & 0.8242 & 0.9957 \\
\hline $\mathrm{TG}(56: 1)$ & -7.892 & -7.973 & 0.8803 & 0.4870 & -5.329 & -5.194 & 0.9751 & 0.9929 \\
\hline $\mathrm{TG}(56: 2)$ & -7.842 & -7.752 & 0.9523 & 0.4870 & -6.426 & -6.456 & 0.7860 & 0.9957 \\
\hline $\mathrm{TG}(56: 2)$ & -8.368 & -8.396 & 0.9153 & 0.4870 & -5.437 & -5.275 & 0.9623 & 0.9929 \\
\hline $\mathrm{TG}(56: 3)$ & -8.257 & -8.265 & 0.0007 & 0.4870 & -7.266 & -7.510 & 0.5492 & 0.9929 \\
\hline $\mathrm{TG}(56: 3)$ & -8.174 & -8.172 & 0.9777 & 0.4870 & -6.478 & -6.460 & 0.5954 & 0.9957 \\
\hline $\mathrm{TG}(56: 3)$ & -9.087 & -8.317 & 0.9980 & 0.4870 & -7.418 & -7.191 & 0.9765 & 0.9929 \\
\hline $\mathrm{TG}(56: 4)$ & -8.770 & -8.580 & 0.1131 & 0.5240 & -6.018 & -5.879 & 0.4504 & 0.9929 \\
\hline $\mathrm{TG}(56: 4)$ & -6.984 & -6.671 & 0.1231 & 0.4870 & -6.066 & -5.902 & 0.6664 & 0.9929 \\
\hline $\mathrm{TG}(56: 4)$ & -9.616 & -9.368 & 0.3841 & 0.4870 & -8.460 & -8.046 & 0.7935 & 0.9702 \\
\hline TG(56:5) & -7.157 & -7.036 & 0.6039 & 0.4870 & -5.385 & -4.972 & 0.4975 & 0.9916 \\
\hline $\mathrm{TG}(56: 5)$ & -7.238 & -6.951 & 0.1578 & 0.4870 & -5.708 & -5.604 & 0.8339 & 0.9929 \\
\hline TG(56:6) & -6.762 & -6.450 & 0.0524 & 0.4870 & -3.873 & -3.877 & 0.9952 & 0.9957 \\
\hline TG(56:7) & -8.882 & -8.347 & 0.2148 & 0.4870 & -5.729 & -5.521 & 0.7647 & 0.9929 \\
\hline TG(56:8) & -10.153 & -9.822 & 0.3461 & 0.4870 & -7.438 & -7.226 & 0.7593 & 0.9929 \\
\hline $\mathrm{TG}(57: 1)$ & -10.772 & -11.213 & 0.5843 & 0.4870 & -8.323 & -7.973 & 0.6981 & 0.9929 \\
\hline $\mathrm{TG}(57: 2)$ & -10.144 & -10.219 & 0.9311 & 0.4870 & -6.828 & -6.579 & 0.7898 & 0.9929 \\
\hline $\mathrm{TG}(57: 3)$ & -10.970 & -10.919 & 0.9368 & 0.4870 & -8.307 & -7.815 & 0.4696 & 0.9867 \\
\hline $\mathrm{TG}(58: 2)$ & -10.634 & -10.778 & 0.8169 & 0.4870 & -8.077 & -7.647 & 0.6374 & 0.9929 \\
\hline TG(58:5) & -9.232 & -8.845 & 0.1368 & 0.4870 & -8.061 & -7.930 & 0.2044 & 0.9929 \\
\hline
\end{tabular}




\begin{tabular}{|c|c|c|c|c|c|c|c|c|}
\hline $\mathrm{TG}(58: 5)$ & -8.810 & -8.312 & 0.1881 & 0.4870 & -8.789 & -8.390 & 0.7572 & 0.8405 \\
\hline $\mathrm{TG}(58: 6)$ & -7.700 & -7.111 & 0.0735 & 0.4870 & -7.218 & -6.806 & 0.1708 & 0.8405 \\
\hline TG(58:7) & -8.027 & -7.454 & 0.0558 & 0.4870 & -7.125 & -6.717 & 0.1401 & 0.8405 \\
\hline $\mathrm{TG}(58: 8)$ & -9.620 & -8.935 & 0.0924 & 0.4870 & -8.249 & -7.820 & 0.4110 & 0.9649 \\
\hline $\mathrm{TG}(59: 3)$ & -11.200 & -10.551 & 0.4624 & 0.5240 & -8.525 & -8.391 & 0.8801 & 0.9929 \\
\hline unknown & -7.494 & -7.034 & 0.0366 & 0.9604 & -7.648 & -7.154 & 0.0122 & 0.5220 \\
\hline unknown & -7.954 & -7.943 & 0.1501 & 0.4870 & -6.519 & -6.492 & 0.0090 & 0.9957 \\
\hline unknown & -6.673 & -6.600 & 0.0050 & 0.4870 & -5.532 & -5.494 & 0.0426 & 0.9929 \\
\hline unknown & -5.294 & -5.276 & 0.1289 & 0.4870 & -5.079 & -4.897 & 0.0457 & 0.6849 \\
\hline unknown & -8.000 & -7.666 & 0.8901 & 0.4870 & -7.479 & -7.276 & 0.0669 & 0.9929 \\
\hline unknown & -8.597 & -7.800 & 0.0836 & 0.4870 & -8.321 & -8.164 & 0.1757 & 0.9649 \\
\hline unknown & -9.058 & -9.142 & 0.0602 & 0.5845 & -8.269 & -7.937 & 0.1499 & 0.9042 \\
\hline unknown & -7.770 & -7.881 & 0.1173 & 0.4870 & -6.854 & -6.722 & 0.1197 & 0.9929 \\
\hline unknown & -7.631 & -7.075 & 0.2129 & 0.5128 & -6.926 & -6.663 & 0.1365 & 0.9042 \\
\hline unknown & -5.347 & -5.011 & 0.3996 & 0.4870 & -4.378 & -4.203 & 0.1351 & 0.9916 \\
\hline unknown & -5.684 & -5.512 & 0.7206 & 0.4870 & -5.189 & -4.874 & 0.1408 & 0.8405 \\
\hline unknown & -5.657 & -5.157 & 0.7442 & 0.4870 & -5.223 & -4.882 & 0.1585 & 0.4306 \\
\hline unknown & -8.610 & -7.490 & 0.1448 & 0.4870 & -9.078 & -8.604 & 0.2311 & 0.8405 \\
\hline unknown & -8.147 & -8.156 & 0.3697 & 0.4870 & -7.690 & -7.647 & 0.2335 & 0.9929 \\
\hline unknown & -5.996 & -5.844 & 0.0349 & 0.4870 & -5.621 & -5.458 & 0.2966 & 0.9916 \\
\hline unknown & -7.472 & -7.234 & 0.7521 & 0.4870 & -6.986 & -6.839 & 0.3008 & 0.8968 \\
\hline unknown & -4.712 & -4.753 & 0.7149 & 0.4870 & -4.511 & -4.335 & 0.3150 & 0.8405 \\
\hline unknown & -7.722 & -7.307 & 0.0774 & 0.4870 & -8.346 & -8.475 & 0.3243 & 0.9929 \\
\hline unknown & -6.789 & -6.706 & 0.1701 & 0.4870 & -6.363 & -6.501 & 0.3274 & 0.9103 \\
\hline unknown & -6.824 & -6.411 & 0.0274 & 0.4870 & -7.270 & -7.311 & 0.4239 & 0.9929 \\
\hline unknown & -7.436 & -6.981 & 0.2204 & 0.4870 & -7.497 & -7.214 & 0.4917 & 0.8968 \\
\hline unknown & -8.840 & -8.216 & 0.5929 & 0.7908 & -7.931 & -7.466 & 0.4966 & 0.4306 \\
\hline unknown & -8.837 & -7.969 & 0.0345 & 0.4870 & -7.860 & -7.602 & 0.8447 & 0.5220 \\
\hline unknown & -6.929 & -6.407 & 0.0347 & 0.5161 & -7.109 & -6.937 & 0.7981 & 0.9172 \\
\hline unknown & -4.182 & -3.950 & 0.0427 & 0.5191 & -3.466 & -3.373 & 0.6979 & 0.9929 \\
\hline unknown & -8.063 & -7.336 & 0.1706 & 0.4870 & -8.623 & -8.295 & 0.7460 & 0.8405 \\
\hline unknown & -8.169 & -7.711 & 0.1822 & 0.9228 & -7.787 & -7.463 & 0.7141 & 0.8405 \\
\hline unknown & -5.630 & -5.000 & 0.2374 & 0.4870 & -6.506 & -6.435 & 0.6175 & 0.9929 \\
\hline unknown & -4.442 & -3.456 & 0.4413 & 0.8255 & -5.423 & -5.219 & 0.5378 & 0.9929 \\
\hline unknown & -7.929 & -7.589 & 0.4543 & 0.4870 & -10.632 & -10.947 & 0.5370 & 0.9929 \\
\hline unknown & -7.842 & -7.345 & 0.5610 & 0.4870 & -8.245 & -8.031 & 0.6670 & 0.9172 \\
\hline unknown & -8.067 & -7.832 & 0.8664 & 0.4900 & -7.509 & -7.293 & 0.8886 & 0.8405 \\
\hline unknown & -8.132 & -8.044 & 0.9684 & 0.8078 & -7.716 & -7.403 & 0.7206 & 0.8405 \\
\hline unknown & -8.515 & -8.274 & 0.9690 & 0.4870 & -10.266 & -10.521 & 0.9389 & 0.9929 \\
\hline
\end{tabular}

${ }^{\mathrm{a}}$ The ambiguous subspecies indicates that the lipidomic profiling gave two possible identifications of the lipid. ${ }^{\mathrm{b}}$ Abbreviations: Cer, ceramide. SM, sphingomyelin. TG, triacylglycerol. DG, diacylglycerol. LysoPC, lysophophosphatidylcholine. LysoPE, lysophosphatidylethanolamine. PC, phosphatidylcholine. PE, phosphatidylethanolamine. PI, phosphatidylinositol. 
Online Resource Table S4

Results from the analysis of variance between the control group (CON) and the high-energy feeding group (HIGH) within time points in liver negative electrospray ionization mode (ESI-) dataset. $P$-values were obtained from the analysis performed using MIXED procedure in SAS, with diet as the fixed effect and pair as the random effect. Adjusted- $p$ values were obtained from $p$-values after false discovery rate control.

\begin{tabular}{|c|c|c|c|c|c|c|c|c|}
\hline \multirow[b]{2}{*}{ Lipid subspecies } & \multicolumn{4}{|c|}{$-8 \mathrm{~d}$} & \multicolumn{4}{|c|}{$9 d$} \\
\hline & $\mathrm{CON}$ & HIGH & $p$-value & Adjusted- $p$ & $\mathrm{CON}$ & HIGH & $p$-value & Adjusted- $p$ \\
\hline$\overline{\operatorname{Cer}(\mathrm{d} 17: 1 / 22: 0)}$ & -4.557 & -4.345 & 0.4093 & 0.7660 & -4.170 & -4.111 & 0.4387 & 0.7766 \\
\hline Cer(d18:1/23:1) & -4.046 & -3.864 & 0.2118 & 0.6124 & -4.303 & -4.300 & 0.9782 & 0.9920 \\
\hline Cer(d18:0/16:0) & -7.126 & -7.310 & 0.5277 & 0.8319 & -6.710 & -6.620 & 0.7984 & 0.9174 \\
\hline Cer(d18:0/18:0) & -9.253 & -9.115 & 0.3444 & 0.7217 & -9.102 & -9.152 & 0.7774 & 0.9094 \\
\hline Cer(d18:0/23:0) & -7.199 & -7.107 & 0.7302 & 0.8936 & -6.123 & -6.365 & 0.5505 & 0.8143 \\
\hline Cer(d18:0/24:0) & -6.907 & -6.821 & 0.7229 & 0.8936 & -6.131 & -6.473 & 0.4314 & 0.7766 \\
\hline Cer(d18:1/16:0) & -3.432 & -3.575 & 0.4802 & 0.8099 & -3.748 & -3.842 & 0.7270 & 0.8912 \\
\hline Cer(d18:1/18:0) & -4.582 & -4.470 & 0.4437 & 0.7913 & -5.057 & -4.749 & 0.3498 & 0.7373 \\
\hline Cer(d18:1/19:0) & -7.442 & -7.354 & 0.4464 & 0.7918 & -7.560 & -7.495 & 0.6083 & 0.8363 \\
\hline Cer(d18:1/20:0) & -5.773 & -5.499 & 0.3928 & 0.7555 & -5.433 & -5.385 & 0.6217 & 0.8363 \\
\hline Cer(d18:1/22:0) & -2.737 & -2.657 & 0.5607 & 0.8347 & -2.468 & -2.481 & 0.9167 & 0.9609 \\
\hline Cer(d18:1/23:0) & -2.077 & -1.894 & 0.1116 & 0.5435 & -1.758 & -1.737 & 0.8667 & 0.9379 \\
\hline Cer(d18:1/24:0) & -1.848 & -1.758 & 0.5652 & 0.8349 & -1.618 & -1.710 & 0.6359 & 0.8408 \\
\hline Cer(d18:1/24:1) & -3.756 & -3.400 & 0.0402 & 0.4977 & -3.658 & -3.547 & 0.5549 & 0.8191 \\
\hline Cer(d18:1/25:0) & -3.808 & -3.627 & 0.2225 & 0.6171 & -3.869 & -4.195 & 0.2417 & 0.6723 \\
\hline Cer(d18:1/26:0) & -3.477 & -3.222 & 0.2691 & 0.6519 & -4.805 & -4.437 & 0.4508 & 0.7820 \\
\hline Cer(d18:2/26:0) & -6.217 & -6.070 & 0.5876 & 0.8433 & -7.412 & -7.460 & 0.8873 & 0.9490 \\
\hline Cer(d18:2/16:0) & -7.020 & -7.057 & 0.7704 & 0.9050 & -7.812 & -7.606 & 0.4651 & 0.7838 \\
\hline Cer(d18:2/18:0) & -9.002 & -9.007 & 0.9845 & 0.9905 & -9.789 & -9.672 & 0.7038 & 0.8769 \\
\hline Cer(d18:2/20:0) & -10.068 & -9.907 & 0.3263 & 0.7018 & -10.583 & -10.453 & 0.5183 & 0.7975 \\
\hline Cer(d18:2/22:0) & -5.498 & -5.272 & 0.2632 & 0.6445 & -5.844 & -5.704 & 0.4783 & 0.7846 \\
\hline Cer(d18:2/23:1) & -8.184 & -7.893 & 0.1763 & 0.5904 & -9.273 & -9.011 & 0.1045 & 0.5450 \\
\hline Cer(d18:2/24:1) & -6.884 & -6.225 & 0.0414 & 0.4977 & -7.198 & -7.030 & 0.3590 & 0.7407 \\
\hline Cer(d18:1/25:1) & -5.308 & -4.906 & 0.0492 & 0.4977 & -5.462 & -5.342 & 0.5099 & 0.7935 \\
\hline Cer(d18:2/26:01) & -10.620 & -10.307 & 0.1936 & 0.5980 & -11.634 & -11.395 & 0.4653 & 0.7838 \\
\hline HexCer(d18:1/16:0) & -9.193 & -9.841 & 0.0739 & 0.5174 & -9.589 & -9.764 & 0.6619 & 0.8550 \\
\hline HexCer(d18:1/22:0) & -7.516 & -7.658 & 0.4627 & 0.7999 & -7.518 & -7.396 & 0.4897 & 0.7897 \\
\hline HexCer(d18:1/23:0) & -7.698 & -7.590 & 0.5416 & 0.8347 & -7.445 & -7.253 & 0.1995 & 0.6305 \\
\hline HexCer(d18:1/24:0) & -7.173 & -7.031 & 0.4526 & 0.7964 & -7.468 & -7.204 & 0.1564 & 0.5918 \\
\hline HexCer(d18:1/24:1) & -10.064 & -9.887 & 0.4997 & 0.8203 & -10.348 & -10.322 & 0.9163 & 0.9609 \\
\hline HexCer(d18:2/20:0) & -8.585 & -8.444 & 0.5070 & 0.8248 & -10.849 & -10.871 & 0.9663 & 0.9879 \\
\hline LysoPC(18:0) & -3.519 & -3.564 & 0.6651 & 0.8674 & -3.391 & -3.329 & 0.5585 & 0.8217 \\
\hline LysoPE(18:0) & -5.455 & -5.081 & 0.2380 & 0.6252 & -4.810 & -4.482 & 0.0596 & 0.4631 \\
\hline $\mathrm{PC}(16: 0 / 16: 1)+$ & -6.415 & -5.387 & 0.0823 & 0.5174 & -6.565 & -6.565 & 0.9997 & 0.9997 \\
\hline \multicolumn{9}{|l|}{$\operatorname{PC}(14: 0 / 18: 1)^{\mathrm{a}}$} \\
\hline $\mathrm{PC}(16: 0 / 20: 4)$ & -6.087 & -5.481 & 0.0880 & 0.5174 & -6.656 & -6.252 & 0.2499 & 0.6776 \\
\hline $\mathrm{PC}(18: 1 / 20: 1)$ & -5.222 & -4.859 & 0.1999 & 0.6008 & -5.540 & -5.259 & 0.1562 & 0.5918 \\
\hline $\mathrm{PC}(30: 0)$ & -6.412 & -5.101 & 0.1312 & 0.5584 & -5.928 & -4.897 & 0.1230 & 0.5532 \\
\hline $\mathrm{PC}(33: 0)$ & -6.035 & -5.021 & 0.1309 & 0.5584 & -6.993 & -6.317 & 0.1308 & 0.5621 \\
\hline $\mathrm{PC}(33: 1)$ & -8.015 & -6.847 & 0.0231 & 0.4977 & -8.439 & -7.703 & 0.0583 & 0.4631 \\
\hline $\mathrm{PC}(36: 2)$ & -7.495 & -6.695 & 0.1567 & 0.5769 & -8.359 & -8.194 & 0.5325 & 0.8034 \\
\hline $\mathrm{PC}(38: 3)$ & -6.784 & -6.808 & 0.9491 & 0.9794 & -7.736 & -8.115 & 0.3175 & 0.7186 \\
\hline $\operatorname{PE}(16: 0 / 20: 4)+$ & -3.624 & -3.150 & 0.4035 & 0.7649 & -2.828 & -2.379 & 0.3920 & 0.7599 \\
\hline \multicolumn{9}{|l|}{$\operatorname{PE}(18: 2 / 18: 2)^{a}$} \\
\hline $\operatorname{PE}(18: 0 / 18: 1)$ & -2.834 & -2.882 & 0.8025 & 0.9124 & -2.693 & -2.590 & 0.5960 & 0.8363 \\
\hline $\operatorname{PE}(18: 0 / 20: 3)$ & -2.389 & -1.543 & 0.0635 & 0.4993 & -2.758 & -2.653 & 0.8143 & 0.9244 \\
\hline $\operatorname{PE}(18: 0 / 22: 4)$ & -5.128 & -3.507 & 0.0441 & 0.4977 & -5.955 & -4.966 & 0.0602 & 0.4631 \\
\hline $\operatorname{PE}(18: 1 / 18: 0)$ & -0.975 & -0.496 & 0.1117 & 0.5435 & -0.793 & -0.492 & 0.0505 & 0.4558 \\
\hline $\operatorname{PE}(32: 0)$ & -9.387 & -9.364 & 0.9001 & 0.9596 & -9.669 & -9.548 & 0.6237 & 0.8363 \\
\hline $\operatorname{PE}(34: 1)$ & -2.012 & -1.965 & 0.8976 & 0.9591 & -1.239 & -1.082 & 0.4674 & 0.7838 \\
\hline
\end{tabular}




\begin{tabular}{|c|c|c|c|c|c|c|c|c|}
\hline $\operatorname{PE}(36: 2)$ & -0.973 & -0.470 & 0.0696 & 0.5064 & -0.270 & 0.013 & 0.3467 & 0.7373 \\
\hline $\operatorname{PE}(36: 2 e)$ & -5.916 & -5.366 & 0.0364 & 0.4977 & -5.584 & -5.371 & 0.2498 & 0.6776 \\
\hline $\operatorname{PE}(36: 3)$ & -3.582 & -2.613 & 0.0376 & 0.4977 & -2.808 & -2.328 & 0.2596 & 0.6791 \\
\hline $\operatorname{PE}(36: 3 e)$ & -5.824 & -5.000 & 0.0508 & 0.4977 & -4.991 & -4.740 & 0.6027 & 0.8363 \\
\hline PE(38:4) & -3.553 & -2.508 & 0.0812 & 0.5174 & -4.727 & -4.354 & 0.6391 & 0.8413 \\
\hline $\operatorname{PE}(38: 4 e)$ & -7.914 & -7.130 & 0.0606 & 0.4984 & -8.225 & -8.006 & 0.5991 & 0.8363 \\
\hline $\operatorname{PE}(38: 5 e)$ & -5.048 & -4.193 & 0.2224 & 0.6171 & -4.957 & -4.444 & 0.4652 & 0.7838 \\
\hline PE(p16:0/18:1) & -4.797 & -3.628 & 0.0070 & 0.4977 & -3.938 & -3.350 & 0.0170 & 0.4440 \\
\hline PE(p16:0/18:2) & -4.819 & -3.422 & 0.0104 & 0.4977 & -3.906 & -3.236 & 0.0512 & 0.4558 \\
\hline $\mathrm{PE}(\mathrm{P}-16: 0 / 20: 4)$ & -5.381 & -4.431 & 0.1662 & 0.5796 & -5.462 & -4.675 & 0.1920 & 0.6292 \\
\hline $\mathrm{PE}(\mathrm{P}-16: 0 / 22: 6)$ & -6.621 & -5.022 & 0.0807 & 0.5174 & -7.056 & -5.759 & 0.2058 & 0.6337 \\
\hline PI(40:6) & -8.018 & -7.084 & 0.1643 & 0.5796 & -8.275 & -7.161 & 0.0427 & 0.4440 \\
\hline $\mathrm{SM}(\mathrm{d} 18: 1 / 16: 0)$ & -4.863 & -5.009 & 0.2205 & 0.6171 & -5.248 & -5.077 & 0.5332 & 0.8034 \\
\hline unknown & -8.008 & -7.827 & 0.6279 & 0.8514 & -8.237 & -7.724 & 0.0780 & 0.5026 \\
\hline unknown & -7.755 & -8.182 & 0.1452 & 0.5602 & -7.952 & -7.769 & 0.3526 & 0.7373 \\
\hline unknown & 5.308 & 4.292 & 0.5909 & 0.8433 & 6.498 & 4.967 & 0.3370 & 0.7315 \\
\hline unknown & -7.734 & -8.112 & 0.0553 & 0.4977 & -8.130 & -7.834 & 0.3508 & 0.7373 \\
\hline unknown & -7.066 & -7.650 & 0.1269 & 0.5568 & -7.696 & -7.533 & 0.6205 & 0.8363 \\
\hline unknown & 4.965 & 3.724 & 0.4122 & 0.7674 & 6.519 & 4.810 & 0.1661 & 0.5944 \\
\hline unknown & 5.574 & 5.448 & 0.5703 & 0.8363 & 6.009 & 6.249 & 0.3469 & 0.7373 \\
\hline unknown & 9.803 & 9.830 & 0.6039 & 0.8495 & 9.952 & 9.989 & 0.6044 & 0.8363 \\
\hline unknown & -5.538 & -5.585 & 0.8346 & 0.9272 & -5.609 & -5.433 & 0.2497 & 0.6776 \\
\hline unknown & 5.505 & 4.355 & 0.4983 & 0.8203 & 6.218 & 4.912 & 0.2857 & 0.6954 \\
\hline unknown & 6.749 & 6.996 & 0.0332 & 0.4977 & 7.376 & 7.401 & 0.8942 & 0.9524 \\
\hline unknown & 6.209 & 6.165 & 0.7478 & 0.9005 & 4.426 & 5.297 & 0.2536 & 0.6776 \\
\hline unknown & -7.434 & -7.948 & 0.1143 & 0.5435 & -7.693 & -7.620 & 0.6494 & 0.8471 \\
\hline unknown & 8.067 & 8.084 & 0.8886 & 0.9542 & 7.801 & 7.873 & 0.3441 & 0.7373 \\
\hline unknown & 10.340 & 10.359 & 0.2952 & 0.6726 & 10.351 & 10.377 & 0.7321 & 0.8944 \\
\hline unknown & -7.883 & -7.052 & 0.1015 & 0.5435 & -8.558 & -8.168 & 0.3064 & 0.7132 \\
\hline unknown & -8.267 & -8.554 & 0.3061 & 0.6837 & -7.990 & -7.654 & 0.1181 & 0.5479 \\
\hline unknown & 6.497 & 6.781 & 0.1405 & 0.5584 & 7.172 & 7.251 & 0.7877 & 0.9116 \\
\hline unknown & -8.024 & -8.904 & 0.0689 & 0.5054 & -8.404 & -8.136 & 0.4710 & 0.7838 \\
\hline unknown & -7.527 & -7.506 & 0.9422 & 0.9756 & -7.960 & -7.592 & 0.1530 & 0.5918 \\
\hline unknown & -8.271 & -8.777 & 0.1023 & 0.5435 & -8.383 & -8.245 & 0.5020 & 0.7924 \\
\hline unknown & -6.567 & -7.022 & 0.2023 & 0.6011 & -6.917 & -6.541 & 0.2661 & 0.6882 \\
\hline unknown & -7.102 & -7.442 & 0.3212 & 0.6965 & -8.395 & -8.112 & 0.5028 & 0.7926 \\
\hline unknown & 8.973 & 8.864 & 0.4616 & 0.7999 & 9.108 & 9.129 & 0.7697 & 0.9073 \\
\hline unknown & 10.544 & 10.494 & 0.2587 & 0.6427 & 10.407 & 10.406 & 0.9871 & 0.9969 \\
\hline unknown & 6.258 & 7.300 & 0.0020 & 0.4977 & 5.618 & 5.721 & 0.4724 & 0.7838 \\
\hline unknown & 6.581 & 6.737 & 0.4545 & 0.7965 & 6.894 & 6.892 & 0.9930 & 0.9990 \\
\hline unknown & -8.182 & -7.563 & 0.2420 & 0.6276 & -8.717 & -8.078 & 0.0788 & 0.5026 \\
\hline unknown & 8.139 & 8.684 & 0.0335 & 0.4977 & 7.222 & 7.299 & 0.8741 & 0.9406 \\
\hline unknown & -7.689 & -7.600 & 0.5325 & 0.8341 & -7.724 & -7.282 & 0.0313 & 0.4440 \\
\hline unknown & -6.813 & -7.332 & 0.1051 & 0.5435 & -6.803 & -6.612 & 0.3509 & 0.7373 \\
\hline unknown & -8.839 & -8.188 & 0.1946 & 0.5980 & -8.620 & -7.849 & 0.0239 & 0.4440 \\
\hline unknown & -7.074 & -7.229 & 0.5774 & 0.8417 & -7.100 & -6.730 & 0.0702 & 0.4936 \\
\hline unknown & 7.226 & 7.410 & 0.2623 & 0.6445 & 7.156 & 7.068 & 0.5428 & 0.8057 \\
\hline unknown & -7.298 & -7.433 & 0.6848 & 0.8784 & -7.253 & -6.977 & 0.2900 & 0.7019 \\
\hline unknown & 8.805 & 9.021 & 0.0484 & 0.4977 & 8.501 & 8.418 & 0.6744 & 0.8610 \\
\hline unknown & -4.538 & -4.567 & 0.8092 & 0.9148 & -4.483 & -4.179 & 0.0691 & 0.4920 \\
\hline unknown & 6.522 & 5.824 & 0.0139 & 0.4977 & 6.427 & 6.239 & 0.4587 & 0.7838 \\
\hline unknown & -6.867 & -7.160 & 0.2596 & 0.6427 & -7.209 & -6.785 & 0.0737 & 0.4951 \\
\hline unknown & -8.418 & -8.482 & 0.6824 & 0.8771 & -8.354 & -8.135 & 0.1418 & 0.5706 \\
\hline unknown & 6.792 & 6.582 & 0.1033 & 0.5435 & 5.882 & 5.844 & 0.8910 & 0.9513 \\
\hline unknown & 8.533 & 8.835 & 0.0516 & 0.4977 & 8.057 & 7.946 & 0.7040 & 0.8769 \\
\hline unknown & -8.555 & -9.605 & 0.0622 & 0.4993 & -8.472 & -8.159 & 0.4577 & 0.7838 \\
\hline unknown & -7.942 & -7.855 & 0.7988 & 0.9122 & -7.902 & -7.560 & 0.0312 & 0.4440 \\
\hline
\end{tabular}




\begin{tabular}{|c|c|c|c|c|c|c|c|c|}
\hline unknown & 6.896 & 7.021 & 0.1866 & 0.5904 & 6.959 & 7.032 & 0.5310 & 0.8034 \\
\hline unknown & 7.105 & 7.131 & 0.8029 & 0.9124 & 7.293 & 7.453 & 0.0363 & 0.4440 \\
\hline unknown & -5.356 & -5.369 & 0.8945 & 0.9573 & -5.234 & -4.844 & 0.0171 & 0.4440 \\
\hline unknown & 8.983 & 8.906 & 0.3936 & 0.7555 & 8.969 & 9.073 & 0.2756 & 0.6923 \\
\hline unknown & 7.378 & 7.406 & 0.7726 & 0.9067 & 7.293 & 7.384 & 0.5021 & 0.7924 \\
\hline unknown & -6.910 & -7.037 & 0.6614 & 0.8643 & -7.000 & -6.709 & 0.2054 & 0.6337 \\
\hline unknown & 6.251 & 6.638 & 0.0750 & 0.5174 & 6.670 & 6.611 & 0.8339 & 0.9282 \\
\hline unknown & -8.441 & -8.786 & 0.4587 & 0.7999 & -8.764 & -8.616 & 0.5916 & 0.8363 \\
\hline unknown & 5.870 & 6.197 & 0.1569 & 0.5769 & 5.988 & 5.960 & 0.9247 & 0.9645 \\
\hline unknown & 6.709 & 7.234 & 0.1788 & 0.5904 & 5.776 & 5.836 & 0.7822 & 0.9094 \\
\hline unknown & -7.614 & -7.902 & 0.0689 & 0.5054 & -7.350 & -7.445 & 0.6003 & 0.8363 \\
\hline unknown & 7.190 & 7.206 & 0.9333 & 0.9754 & 6.911 & 6.985 & 0.5062 & 0.7935 \\
\hline unknown & 9.407 & 9.391 & 0.8379 & 0.9283 & 9.286 & 9.345 & 0.5987 & 0.8363 \\
\hline unknown & 7.387 & 7.416 & 0.7184 & 0.8935 & 7.128 & 7.164 & 0.8511 & 0.9294 \\
\hline unknown & -8.663 & -7.954 & 0.1217 & 0.5464 & -9.141 & -9.166 & 0.9215 & 0.9637 \\
\hline unknown & 6.841 & 6.963 & 0.2265 & 0.6171 & 6.650 & 6.800 & 0.4369 & 0.7766 \\
\hline unknown & 7.816 & 7.663 & 0.2961 & 0.6726 & 8.004 & 7.638 & 0.0069 & 0.4440 \\
\hline unknown & 8.079 & 7.902 & 0.2331 & 0.6207 & 8.101 & 8.255 & 0.2026 & 0.6332 \\
\hline unknown & 6.432 & 6.547 & 0.0815 & 0.5174 & 6.315 & 6.222 & 0.6433 & 0.8424 \\
\hline unknown & 9.577 & 9.551 & 0.6593 & 0.8635 & 9.339 & 9.377 & 0.8305 & 0.9282 \\
\hline unknown & 7.536 & 7.570 & 0.8349 & 0.9272 & 7.625 & 7.481 & 0.2294 & 0.6588 \\
\hline unknown & 6.122 & 6.112 & 0.9447 & 0.9767 & 6.308 & 6.336 & 0.9131 & 0.9609 \\
\hline unknown & 7.361 & 7.806 & 0.0316 & 0.4977 & 6.679 & 6.699 & 0.9558 & 0.9803 \\
\hline unknown & 8.183 & 9.214 & 0.0073 & 0.4977 & 7.223 & 7.871 & 0.1364 & 0.5638 \\
\hline unknown & -7.969 & -8.533 & 0.1303 & 0.5584 & -8.025 & -7.766 & 0.2807 & 0.6923 \\
\hline unknown & -9.423 & -8.863 & 0.3044 & 0.6835 & -9.245 & -8.506 & 0.0166 & 0.4440 \\
\hline unknown & -7.976 & -8.234 & 0.4307 & 0.7785 & -8.119 & -7.910 & 0.3318 & 0.7310 \\
\hline unknown & 6.404 & 6.731 & 0.0663 & 0.5051 & 6.186 & 6.340 & 0.3257 & 0.7272 \\
\hline unknown & -8.207 & -8.508 & 0.4143 & 0.7674 & -8.241 & -7.924 & 0.2788 & 0.6923 \\
\hline unknown & 8.267 & 8.460 & 0.0126 & 0.4977 & 7.845 & 7.778 & 0.7780 & 0.9094 \\
\hline unknown & 8.375 & 8.564 & 0.4872 & 0.8112 & 8.277 & 8.183 & 0.3844 & 0.7540 \\
\hline unknown & 8.006 & 8.192 & 0.1948 & 0.5980 & 7.299 & 7.341 & 0.9206 & 0.9635 \\
\hline unknown & 6.328 & 6.341 & 0.9308 & 0.9749 & 6.563 & 6.694 & 0.2819 & 0.6923 \\
\hline unknown & -7.587 & -8.025 & 0.0348 & 0.4977 & -7.315 & -7.432 & 0.5631 & 0.8256 \\
\hline unknown & 8.166 & 8.067 & 0.3945 & 0.7555 & 8.017 & 8.132 & 0.4032 & 0.7702 \\
\hline unknown & 6.835 & 6.788 & 0.6507 & 0.8622 & 6.605 & 6.651 & 0.7562 & 0.9020 \\
\hline unknown & -7.956 & -8.222 & 0.3578 & 0.7286 & -8.232 & -7.932 & 0.0434 & 0.4440 \\
\hline unknown & 6.477 & 6.429 & 0.7568 & 0.9026 & 6.223 & 6.326 & 0.4521 & 0.7820 \\
\hline unknown & 8.593 & 8.528 & 0.5953 & 0.8453 & 8.460 & 8.433 & 0.8671 & 0.9379 \\
\hline unknown & 6.919 & 6.824 & 0.2408 & 0.6276 & 6.516 & 6.612 & 0.7075 & 0.8771 \\
\hline unknown & -8.741 & -7.864 & 0.0471 & 0.4977 & -8.242 & -7.946 & 0.0988 & 0.5380 \\
\hline unknown & -6.938 & -5.974 & 0.1867 & 0.5904 & -6.707 & -5.737 & 0.0479 & 0.4558 \\
\hline unknown & -5.175 & -4.881 & 0.3195 & 0.6958 & -4.708 & -4.786 & 0.6305 & 0.8380 \\
\hline unknown & -6.868 & -6.552 & 0.5280 & 0.8319 & -6.110 & -5.527 & 0.2356 & 0.6658 \\
\hline unknown & -7.873 & -7.122 & 0.1476 & 0.5621 & -7.946 & -7.197 & 0.1603 & 0.5933 \\
\hline unknown & -5.228 & -4.669 & 0.2562 & 0.6427 & -5.044 & -4.405 & 0.1511 & 0.5918 \\
\hline unknown & -3.954 & -3.818 & 0.6284 & 0.8514 & -3.695 & -3.550 & 0.6198 & 0.8363 \\
\hline unknown & -5.394 & -5.281 & 0.5894 & 0.8433 & -5.194 & -5.098 & 0.4929 & 0.7901 \\
\hline unknown & -6.936 & -6.291 & 0.2508 & 0.6379 & -7.041 & -6.107 & 0.1146 & 0.5479 \\
\hline unknown & -7.273 & -6.593 & 0.1038 & 0.5435 & -7.560 & -7.329 & 0.5013 & 0.7924 \\
\hline unknown & -4.648 & -4.240 & 0.0712 & 0.5124 & -4.727 & -4.755 & 0.9166 & 0.9609 \\
\hline unknown & -5.976 & -5.512 & 0.0765 & 0.5174 & -6.101 & -6.131 & 0.9239 & 0.9645 \\
\hline unknown & -7.518 & -7.010 & 0.0319 & 0.4977 & -7.599 & -7.374 & 0.4664 & 0.7838 \\
\hline unknown & -7.792 & -7.625 & 0.4417 & 0.7899 & -7.401 & -7.217 & 0.5001 & 0.7924 \\
\hline unknown & -7.870 & -8.129 & 0.5554 & 0.8347 & -8.299 & -7.931 & 0.5927 & 0.8363 \\
\hline unknown & -5.669 & -5.962 & 0.1836 & 0.5904 & -5.606 & -5.583 & 0.8363 & 0.9282 \\
\hline unknown & -4.831 & -5.120 & 0.3643 & 0.7303 & -5.260 & -5.282 & 0.7074 & 0.8771 \\
\hline
\end{tabular}




\begin{tabular}{|c|c|c|c|c|c|c|c|c|}
\hline unknown & -6.172 & -6.673 & 0.2219 & 0.6171 & -6.665 & -6.824 & 0.3780 & 0.7525 \\
\hline unknown & -7.199 & -7.596 & 0.2172 & 0.6150 & -7.214 & -7.111 & 0.2569 & 0.6789 \\
\hline unknown & -7.025 & -7.252 & 0.4762 & 0.8080 & -7.340 & -7.007 & 0.4445 & 0.7795 \\
\hline unknown & -8.400 & -6.815 & 0.0409 & 0.4977 & -8.799 & -7.532 & 0.0711 & 0.4936 \\
\hline unknown & -7.896 & -6.808 & 0.1370 & 0.5584 & -8.005 & -7.071 & 0.0792 & 0.5026 \\
\hline unknown & -8.075 & -6.897 & 0.1196 & 0.5435 & -7.789 & -6.796 & 0.0369 & 0.4440 \\
\hline unknown & -8.337 & -7.989 & 0.6692 & 0.8702 & -8.289 & -8.016 & 0.4373 & 0.7766 \\
\hline unknown & -4.759 & -4.199 & 0.1577 & 0.5769 & -4.657 & -4.539 & 0.6181 & 0.8363 \\
\hline unknown & -7.381 & -6.682 & 0.0412 & 0.4977 & -7.819 & -7.722 & 0.6030 & 0.8363 \\
\hline unknown & -9.209 & -8.458 & 0.0376 & 0.4977 & -9.683 & -9.697 & 0.9342 & 0.9689 \\
\hline unknown & -5.709 & -5.524 & 0.5626 & 0.8347 & -5.739 & -5.333 & 0.1965 & 0.6302 \\
\hline unknown & -7.575 & -7.225 & 0.3632 & 0.7303 & -7.543 & -7.019 & 0.1715 & 0.5944 \\
\hline unknown & -7.334 & -7.488 & 0.4434 & 0.7913 & -7.553 & -7.283 & 0.4928 & 0.7901 \\
\hline unknown & -6.450 & -6.723 & 0.1740 & 0.5904 & -6.569 & -6.493 & 0.4996 & 0.7924 \\
\hline unknown & -7.071 & -7.004 & 0.7998 & 0.9122 & -7.309 & -6.948 & 0.0334 & 0.4440 \\
\hline unknown & -8.359 & -8.340 & 0.9458 & 0.9768 & -8.386 & -8.288 & 0.7497 & 0.9011 \\
\hline unknown & -7.159 & -7.285 & 0.4449 & 0.7918 & -7.020 & -6.869 & 0.4306 & 0.7766 \\
\hline unknown & -8.210 & -7.885 & 0.3480 & 0.7251 & -7.385 & -7.220 & 0.6685 & 0.8590 \\
\hline unknown & -6.783 & -6.675 & 0.7849 & 0.9091 & -6.253 & -5.810 & 0.2118 & 0.6419 \\
\hline unknown & -4.891 & -4.851 & 0.8535 & 0.9366 & -5.066 & -4.789 & 0.1237 & 0.5532 \\
\hline unknown & -8.226 & -8.138 & 0.6111 & 0.8511 & -8.245 & -8.216 & 0.8967 & 0.9536 \\
\hline unknown & -6.614 & -6.471 & 0.5627 & 0.8347 & -6.778 & -6.471 & 0.1502 & 0.5917 \\
\hline unknown & -5.261 & -5.659 & 0.0175 & 0.4977 & -5.463 & -5.276 & 0.3594 & 0.7407 \\
\hline unknown & -7.249 & -7.672 & 0.0911 & 0.5227 & -7.271 & -7.151 & 0.6384 & 0.8413 \\
\hline unknown & -6.976 & -7.306 & 0.1194 & 0.5435 & -7.066 & -6.771 & 0.2984 & 0.7106 \\
\hline unknown & -8.385 & -8.677 & 0.2532 & 0.6427 & -8.837 & -8.808 & 0.9083 & 0.9590 \\
\hline unknown & -5.036 & -5.688 & 0.1488 & 0.5634 & -5.606 & -5.586 & 0.9367 & 0.9689 \\
\hline unknown & -2.318 & -2.270 & 0.8098 & 0.9148 & -2.519 & -2.553 & 0.9148 & 0.9609 \\
\hline unknown & -7.971 & -7.727 & 0.2388 & 0.6254 & -7.694 & -7.419 & 0.0944 & 0.5296 \\
\hline unknown & -6.633 & -6.252 & 0.4140 & 0.7674 & -6.987 & -6.522 & 0.1837 & 0.6159 \\
\hline unknown & -6.383 & -6.911 & 0.3882 & 0.7509 & -6.508 & -6.655 & 0.4835 & 0.7880 \\
\hline unknown & -8.668 & -8.374 & 0.1865 & 0.5904 & -8.307 & -7.879 & 0.0262 & 0.4440 \\
\hline unknown & -5.369 & -6.244 & 0.1752 & 0.5904 & -6.629 & -6.553 & 0.8660 & 0.9379 \\
\hline unknown & 7.584 & 6.650 & 0.2043 & 0.6030 & 7.326 & 6.964 & 0.1635 & 0.5944 \\
\hline unknown & -7.738 & -7.742 & 0.9935 & 0.9965 & -6.708 & -6.464 & 0.4243 & 0.7766 \\
\hline unknown & -5.586 & -6.249 & 0.2583 & 0.6427 & -6.090 & -5.320 & 0.1482 & 0.5876 \\
\hline unknown & -7.217 & -6.716 & 0.0626 & 0.4993 & -6.910 & -6.162 & 0.1624 & 0.5942 \\
\hline unknown & -7.401 & -7.740 & 0.0638 & 0.4993 & -7.510 & -7.315 & 0.2465 & 0.6776 \\
\hline unknown & -7.171 & -7.177 & 0.9668 & 0.9827 & -7.078 & -7.080 & 0.9939 & 0.9990 \\
\hline unknown & -6.131 & -6.436 & 0.4863 & 0.8112 & -5.928 & -5.964 & 0.7457 & 0.8977 \\
\hline unknown & -7.648 & -8.198 & 0.1980 & 0.6008 & -7.739 & -8.435 & 0.1131 & 0.5479 \\
\hline unknown & -8.253 & -8.319 & 0.9378 & 0.9756 & -6.902 & -6.955 & 0.8120 & 0.9244 \\
\hline unknown & -8.138 & -8.993 & 0.0675 & 0.5054 & -9.375 & -9.091 & 0.5089 & 0.7935 \\
\hline unknown & -6.976 & -7.103 & 0.8746 & 0.9476 & -5.824 & -5.981 & 0.6771 & 0.8613 \\
\hline unknown & 6.412 & 5.850 & 0.3971 & 0.7592 & 6.504 & 6.117 & 0.1943 & 0.6302 \\
\hline unknown & -5.805 & -5.619 & 0.2542 & 0.6427 & -5.604 & -5.485 & 0.4614 & 0.7838 \\
\hline unknown & -7.553 & -7.023 & 0.2484 & 0.6362 & -7.389 & -6.707 & 0.0537 & 0.4558 \\
\hline unknown & -7.395 & -6.753 & 0.0583 & 0.4977 & -6.502 & -6.142 & 0.1042 & 0.5450 \\
\hline unknown & -2.431 & -2.508 & 0.3395 & 0.7164 & -2.470 & -2.384 & 0.0285 & 0.4440 \\
\hline unknown & -5.226 & -6.128 & 0.2729 & 0.6563 & -6.802 & -6.638 & 0.7989 & 0.9174 \\
\hline unknown & -6.758 & -6.913 & 0.1360 & 0.5584 & -6.800 & -6.655 & 0.2538 & 0.6776 \\
\hline unknown & -7.673 & -7.796 & 0.7818 & 0.9091 & -7.304 & -7.146 & 0.5094 & 0.7935 \\
\hline unknown & -7.952 & -7.790 & 0.7180 & 0.8935 & -7.027 & -6.832 & 0.5922 & 0.8363 \\
\hline unknown & -7.986 & -8.699 & 0.2603 & 0.6427 & -8.340 & -8.414 & 0.7343 & 0.8944 \\
\hline unknown & -7.477 & -7.169 & 0.3753 & 0.7408 & -6.747 & -6.448 & 0.4186 & 0.7763 \\
\hline unknown & -7.254 & -6.957 & 0.5338 & 0.8341 & -6.483 & -6.344 & 0.6845 & 0.8653 \\
\hline unknown & -5.573 & -5.016 & 0.0173 & 0.4977 & -5.272 & -5.149 & 0.4925 & 0.7901 \\
\hline
\end{tabular}




\begin{tabular}{|c|c|c|c|c|c|c|c|c|}
\hline unknown & -3.607 & -3.436 & 0.5669 & 0.8356 & -3.362 & -3.202 & 0.4730 & 0.7838 \\
\hline unknown & -5.210 & -4.967 & 0.4631 & 0.7999 & -4.982 & -4.795 & 0.0963 & 0.5332 \\
\hline unknown & -7.201 & -7.240 & 0.5541 & 0.8347 & -7.166 & -6.999 & 0.1139 & 0.5479 \\
\hline unknown & -5.992 & -6.049 & 0.5142 & 0.8274 & -6.009 & -5.844 & 0.0456 & 0.4440 \\
\hline unknown & -4.964 & -5.624 & 0.3849 & 0.7475 & -5.259 & -4.989 & 0.7541 & 0.9020 \\
\hline unknown & -6.469 & -6.493 & 0.8620 & 0.9397 & -6.306 & -6.332 & 0.8347 & 0.9282 \\
\hline unknown & -2.539 & -2.694 & 0.5625 & 0.8347 & -2.775 & -2.765 & 0.9489 & 0.9785 \\
\hline unknown & -4.700 & -4.839 & 0.5373 & 0.8347 & -4.768 & -4.644 & 0.5266 & 0.8034 \\
\hline unknown & -6.402 & -6.171 & 0.4147 & 0.7674 & -6.475 & -6.602 & 0.5012 & 0.7924 \\
\hline unknown & -7.167 & -7.191 & 0.9415 & 0.9756 & -7.008 & -7.057 & 0.8074 & 0.9216 \\
\hline unknown & -7.811 & -7.555 & 0.7238 & 0.8936 & -7.717 & -7.303 & 0.1689 & 0.5944 \\
\hline unknown & -7.255 & -7.485 & 0.2278 & 0.6171 & -7.409 & -8.450 & 0.1317 & 0.5629 \\
\hline unknown & -8.153 & -7.855 & 0.5110 & 0.8267 & -8.098 & -8.034 & 0.8405 & 0.9282 \\
\hline unknown & -4.621 & -4.706 & 0.8090 & 0.9148 & -4.521 & -5.972 & 0.0758 & 0.4986 \\
\hline unknown & -6.588 & -6.689 & 0.8199 & 0.9172 & -6.487 & -8.187 & 0.0859 & 0.5105 \\
\hline unknown & -4.926 & -4.751 & 0.6469 & 0.8589 & -4.598 & -4.095 & 0.2263 & 0.6542 \\
\hline unknown & -6.864 & -6.603 & 0.6105 & 0.8511 & -6.318 & -5.932 & 0.3115 & 0.7149 \\
\hline unknown & -5.913 & -5.860 & 0.8524 & 0.9366 & -6.687 & -6.570 & 0.7163 & 0.8847 \\
\hline unknown & -5.373 & -5.535 & 0.6376 & 0.8542 & -5.460 & -5.367 & 0.7979 & 0.9174 \\
\hline unknown & -7.645 & -7.896 & 0.6173 & 0.8511 & -7.862 & -7.848 & 0.9751 & 0.9916 \\
\hline unknown & -2.734 & -2.822 & 0.7452 & 0.9005 & -2.874 & -3.018 & 0.5368 & 0.8037 \\
\hline unknown & -6.087 & -6.270 & 0.6522 & 0.8623 & -5.248 & -5.316 & 0.7999 & 0.9176 \\
\hline unknown & -6.875 & -6.132 & 0.2138 & 0.6142 & -6.386 & -5.991 & 0.1373 & 0.5638 \\
\hline unknown & -7.294 & -7.351 & 0.8016 & 0.9124 & -7.446 & -7.432 & 0.9336 & 0.9689 \\
\hline unknown & 0.999 & 0.991 & 0.9614 & 0.9820 & 1.277 & 1.211 & 0.7219 & 0.8882 \\
\hline unknown & -5.332 & -5.354 & 0.9595 & 0.9820 & -4.668 & -4.867 & 0.5681 & 0.8259 \\
\hline unknown & -6.049 & -6.638 & 0.1587 & 0.5769 & -6.114 & -6.177 & 0.7216 & 0.8882 \\
\hline unknown & -4.103 & -4.053 & 0.7508 & 0.9005 & -3.696 & -3.835 & 0.5359 & 0.8035 \\
\hline unknown & -6.069 & -6.039 & 0.8546 & 0.9366 & -5.734 & -5.862 & 0.3798 & 0.7530 \\
\hline unknown & -8.113 & -7.473 & 0.1768 & 0.5904 & -8.342 & -7.977 & 0.2387 & 0.6695 \\
\hline unknown & -5.658 & -6.036 & 0.1676 & 0.5827 & -5.862 & -5.812 & 0.8574 & 0.9336 \\
\hline unknown & -7.426 & -9.153 & 0.2256 & 0.6171 & -6.411 & -7.185 & 0.1192 & 0.5479 \\
\hline unknown & -2.831 & -2.916 & 0.4800 & 0.8099 & -2.730 & -2.632 & 0.2028 & 0.6332 \\
\hline unknown & -8.906 & -8.568 & 0.4618 & 0.7999 & -7.874 & -7.492 & 0.5137 & 0.7936 \\
\hline unknown & -6.912 & -7.870 & 0.3580 & 0.7286 & -7.018 & -7.123 & 0.8949 & 0.9524 \\
\hline unknown & -8.077 & -7.830 & 0.4702 & 0.8059 & -8.344 & -8.443 & 0.8160 & 0.9244 \\
\hline unknown & -5.016 & -4.898 & 0.7383 & 0.8991 & -4.786 & -4.756 & 0.9420 & 0.9729 \\
\hline unknown & -7.157 & -7.029 & 0.8153 & 0.9172 & -6.910 & -6.848 & 0.9133 & 0.9609 \\
\hline unknown & -4.140 & -3.962 & 0.4880 & 0.8114 & -4.495 & -3.970 & 0.0640 & 0.4834 \\
\hline unknown & -5.319 & -5.244 & 0.6910 & 0.8787 & -5.297 & -5.479 & 0.1065 & 0.5479 \\
\hline unknown & -9.116 & -8.650 & 0.0374 & 0.4977 & -9.273 & -8.516 & 0.0265 & 0.4440 \\
\hline unknown & -7.622 & -8.813 & 0.1917 & 0.5962 & -7.282 & -7.947 & 0.3947 & 0.7608 \\
\hline unknown & -8.256 & -8.968 & 0.4297 & 0.7785 & -8.010 & -8.531 & 0.3673 & 0.7425 \\
\hline unknown & -8.148 & -8.619 & 0.5712 & 0.8363 & -7.926 & -8.321 & 0.6000 & 0.8363 \\
\hline unknown & -5.767 & -6.568 & 0.4712 & 0.8059 & -5.602 & -5.743 & 0.8505 & 0.9294 \\
\hline unknown & -9.245 & -9.735 & 0.6168 & 0.8511 & -9.176 & -9.153 & 0.9704 & 0.9890 \\
\hline unknown & -6.478 & -6.292 & 0.4806 & 0.8099 & -6.370 & -6.312 & 0.7819 & 0.9094 \\
\hline unknown & -8.039 & -8.055 & 0.9531 & 0.9819 & -7.278 & -7.056 & 0.3692 & 0.7429 \\
\hline unknown & 6.301 & 6.992 & 0.1172 & 0.5435 & 6.928 & 6.866 & 0.7963 & 0.9174 \\
\hline unknown & -9.193 & -8.768 & 0.3649 & 0.7303 & -7.472 & -7.294 & 0.7829 & 0.9094 \\
\hline unknown & -8.142 & -7.925 & 0.4944 & 0.8179 & -8.041 & -7.860 & 0.5299 & 0.8034 \\
\hline unknown & -7.141 & -7.076 & 0.8937 & 0.9572 & -5.815 & -5.330 & 0.3072 & 0.7132 \\
\hline unknown & -4.308 & -4.617 & 0.2783 & 0.6587 & -4.173 & -4.036 & 0.3064 & 0.7132 \\
\hline unknown & -8.621 & -9.049 & 0.6889 & 0.8787 & -9.066 & -9.078 & 0.9874 & 0.9969 \\
\hline unknown & -6.495 & -6.247 & 0.2176 & 0.6150 & -6.692 & -6.487 & 0.4290 & 0.7766 \\
\hline unknown & -7.304 & -6.608 & 0.0052 & 0.4977 & -7.585 & -6.713 & 0.0276 & 0.4440 \\
\hline unknown & -4.425 & -4.417 & 0.9756 & 0.9845 & -4.518 & -4.118 & 0.0864 & 0.5105 \\
\hline
\end{tabular}




\begin{tabular}{|c|c|c|c|c|c|c|c|c|}
\hline unknown & -6.243 & -6.201 & 0.9085 & 0.9646 & -6.365 & -5.975 & 0.0996 & 0.5380 \\
\hline unknown & -8.731 & -9.397 & 0.5556 & 0.8347 & -8.639 & -9.014 & 0.7050 & 0.8771 \\
\hline unknown & 8.360 & 8.313 & 0.9136 & 0.9677 & 8.679 & 8.425 & 0.5820 & 0.8339 \\
\hline unknown & 5.881 & 5.861 & 0.9679 & 0.9827 & 6.156 & 5.853 & 0.5911 & 0.8363 \\
\hline unknown & 9.871 & 9.693 & 0.2383 & 0.6252 & 9.970 & 9.745 & 0.1728 & 0.5944 \\
\hline unknown & -7.780 & -7.683 & 0.7619 & 0.9044 & -7.237 & -7.072 & 0.2956 & 0.7057 \\
\hline unknown & 8.441 & 8.323 & 0.7839 & 0.9091 & 8.547 & 8.575 & 0.9336 & 0.9689 \\
\hline unknown & -6.941 & -8.318 & 0.2057 & 0.6030 & -6.935 & -7.857 & 0.3083 & 0.7132 \\
\hline unknown & 8.397 & 8.707 & 0.3946 & 0.7555 & 9.267 & 9.025 & 0.4195 & 0.7763 \\
\hline unknown & -6.148 & -5.818 & 0.4459 & 0.7918 & -5.810 & -5.473 & 0.4306 & 0.7766 \\
\hline unknown & -7.107 & -6.441 & 0.0574 & 0.4977 & -7.150 & -6.685 & 0.0125 & 0.4440 \\
\hline unknown & -4.990 & -4.590 & 0.2780 & 0.6587 & -5.082 & -4.825 & 0.1698 & 0.5944 \\
\hline unknown & -7.140 & -6.627 & 0.2652 & 0.6470 & -7.345 & -7.048 & 0.1585 & 0.5926 \\
\hline unknown & -8.841 & -9.597 & 0.5464 & 0.8347 & -7.687 & -7.920 & 0.5089 & 0.7935 \\
\hline unknown & -7.956 & -8.521 & 0.5606 & 0.8347 & -7.888 & -8.439 & 0.3112 & 0.7149 \\
\hline unknown & -7.181 & -7.715 & 0.6113 & 0.8511 & -7.419 & -7.647 & 0.7588 & 0.9020 \\
\hline unknown & -7.181 & -7.715 & 0.6113 & 0.8511 & -7.419 & -7.647 & 0.7588 & 0.9020 \\
\hline unknown & 10.499 & 10.888 & 0.2010 & 0.6009 & 11.280 & 10.940 & 0.1040 & 0.5450 \\
\hline unknown & -9.135 & -8.307 & 0.0804 & 0.5174 & -8.534 & -8.537 & 0.9973 & 0.9990 \\
\hline unknown & -8.283 & -9.219 & 0.3495 & 0.7260 & -8.235 & -8.998 & 0.4213 & 0.7766 \\
\hline unknown & 9.323 & 9.561 & 0.5919 & 0.8433 & 10.391 & 10.222 & 0.4684 & 0.7838 \\
\hline unknown & 7.058 & 7.403 & 0.5230 & 0.8300 & 8.399 & 8.193 & 0.5906 & 0.8363 \\
\hline unknown & -7.543 & -6.939 & 0.1133 & 0.5435 & -7.191 & -6.603 & 0.0420 & 0.4440 \\
\hline unknown & -8.564 & -8.245 & 0.5593 & 0.8347 & -8.378 & -7.768 & 0.2258 & 0.6542 \\
\hline unknown & -7.435 & -6.596 & 0.0552 & 0.4977 & -7.404 & -6.473 & 0.0272 & 0.4440 \\
\hline unknown & -8.165 & -7.343 & 0.0254 & 0.4977 & -8.364 & -7.955 & 0.0341 & 0.4440 \\
\hline unknown & -7.843 & -7.118 & 0.0460 & 0.4977 & -7.671 & -7.182 & 0.1580 & 0.5926 \\
\hline unknown & 9.796 & 9.852 & 0.3743 & 0.7408 & 9.860 & 9.560 & 0.0716 & 0.4936 \\
\hline unknown & 8.437 & 8.737 & 0.1299 & 0.5584 & 8.306 & 8.098 & 0.1578 & 0.5926 \\
\hline unknown & -7.804 & -8.464 & 0.5451 & 0.8347 & -8.458 & -8.920 & 0.5348 & 0.8035 \\
\hline unknown & 12.526 & 12.792 & 0.1530 & 0.5726 & 13.218 & 12.924 & 0.2126 & 0.6419 \\
\hline unknown & -8.453 & -9.364 & 0.3702 & 0.7376 & -8.155 & -8.914 & 0.3682 & 0.7429 \\
\hline unknown & -7.725 & -8.469 & 0.4816 & 0.8099 & -7.547 & -7.386 & 0.8335 & 0.9282 \\
\hline unknown & 10.072 & 10.491 & 0.0468 & 0.4977 & 11.169 & 10.846 & 0.4548 & 0.7829 \\
\hline unknown & 8.034 & 8.504 & 0.0567 & 0.4977 & 9.280 & 8.838 & 0.2678 & 0.6884 \\
\hline unknown & 8.322 & 8.711 & 0.1318 & 0.5584 & 8.523 & 8.451 & 0.6168 & 0.8363 \\
\hline unknown & -7.485 & -7.089 & 0.3340 & 0.7132 & -8.553 & -8.385 & 0.3303 & 0.7310 \\
\hline unknown & -6.275 & -5.276 & 0.0401 & 0.4977 & -6.641 & -5.645 & 0.0118 & 0.4440 \\
\hline unknown & -6.268 & -5.491 & 0.0526 & 0.4977 & -6.155 & -5.569 & 0.0851 & 0.5105 \\
\hline unknown & 11.033 & 11.230 & 0.4989 & 0.8203 & 10.876 & 10.659 & 0.1836 & 0.6159 \\
\hline unknown & -8.634 & -9.547 & 0.3543 & 0.7286 & -7.814 & -7.718 & 0.8394 & 0.9282 \\
\hline unknown & -7.363 & -7.811 & 0.6534 & 0.8623 & -7.610 & -8.098 & 0.3790 & 0.7530 \\
\hline unknown & -8.355 & -9.022 & 0.4141 & 0.7674 & -8.278 & -8.848 & 0.4412 & 0.7768 \\
\hline unknown & 13.407 & 13.759 & 0.0738 & 0.5174 & 14.165 & 13.958 & 0.4714 & 0.7838 \\
\hline unknown & -7.745 & -9.520 & 0.1854 & 0.5904 & -7.586 & -8.520 & 0.4113 & 0.7754 \\
\hline unknown & -7.231 & -8.395 & 0.3925 & 0.7555 & -7.019 & -8.113 & 0.1968 & 0.6302 \\
\hline unknown & -8.380 & -9.625 & 0.2850 & 0.6648 & -8.528 & -9.451 & 0.2669 & 0.6882 \\
\hline unknown & -8.152 & -9.179 & 0.3040 & 0.6835 & -8.565 & -9.169 & 0.3843 & 0.7540 \\
\hline unknown & 8.661 & 8.457 & 0.5807 & 0.8433 & 9.249 & 9.067 & 0.6117 & 0.8363 \\
\hline unknown & 8.407 & 8.608 & 0.6002 & 0.8478 & 9.564 & 9.069 & 0.0879 & 0.5130 \\
\hline unknown & 9.748 & 9.967 & 0.2271 & 0.6171 & 10.188 & 10.005 & 0.2725 & 0.6908 \\
\hline unknown & 7.189 & 7.475 & 0.1941 & 0.5980 & 7.746 & 7.550 & 0.4405 & 0.7766 \\
\hline unknown & -8.588 & -7.486 & 0.0115 & 0.4977 & -7.785 & -7.085 & 0.0328 & 0.4440 \\
\hline unknown & -9.073 & -9.568 & 0.6929 & 0.8787 & -8.332 & -8.545 & 0.7964 & 0.9174 \\
\hline unknown & -6.172 & -7.077 & 0.6466 & 0.8589 & -5.314 & -5.501 & 0.8405 & 0.9282 \\
\hline unknown & -6.789 & -6.046 & 0.0641 & 0.4993 & -6.246 & -5.378 & 0.0342 & 0.4440 \\
\hline unknown & 8.844 & 9.604 & 0.0701 & 0.5076 & 8.689 & 8.626 & 0.8420 & 0.9282 \\
\hline
\end{tabular}




\begin{tabular}{|c|c|c|c|c|c|c|c|c|}
\hline unknown & -5.236 & -4.473 & 0.0432 & 0.4977 & -5.012 & -4.603 & 0.1696 & 0.5944 \\
\hline unknown & -7.617 & -6.651 & 0.0559 & 0.4977 & -7.379 & -6.900 & 0.2446 & 0.6774 \\
\hline unknown & 11.252 & 11.733 & 0.1658 & 0.5796 & 11.601 & 11.737 & 0.6913 & 0.8700 \\
\hline unknown & -8.483 & -9.501 & 0.3014 & 0.6791 & -7.984 & -8.588 & 0.4293 & 0.7766 \\
\hline unknown & -7.353 & -8.041 & 0.5461 & 0.8347 & -7.975 & -8.756 & 0.3252 & 0.7272 \\
\hline unknown & 6.955 & 6.719 & 0.6157 & 0.8511 & 7.082 & 6.788 & 0.4257 & 0.7766 \\
\hline unknown & 13.752 & 13.941 & 0.2922 & 0.6709 & 14.332 & 13.899 & 0.1906 & 0.6261 \\
\hline unknown & -6.463 & -7.576 & 0.3596 & 0.7286 & -6.513 & -6.936 & 0.7004 & 0.8740 \\
\hline unknown & 8.676 & 9.045 & 0.1818 & 0.5904 & 8.693 & 8.520 & 0.3065 & 0.7132 \\
\hline unknown & 9.693 & 9.581 & 0.7517 & 0.9005 & 10.501 & 10.311 & 0.6152 & 0.8363 \\
\hline unknown & 8.762 & 9.019 & 0.3453 & 0.7217 & 9.644 & 8.973 & 0.0353 & 0.4440 \\
\hline unknown & 7.694 & 7.942 & 0.3166 & 0.6958 & 8.116 & 7.995 & 0.5425 & 0.8057 \\
\hline unknown & 10.661 & 10.901 & 0.2909 & 0.6709 & 11.157 & 11.008 & 0.2350 & 0.6658 \\
\hline unknown & 8.394 & 8.805 & 0.2253 & 0.6171 & 9.175 & 8.921 & 0.2409 & 0.6719 \\
\hline unknown & -9.990 & -8.669 & 0.0145 & 0.4977 & -9.258 & -8.419 & 0.0182 & 0.4440 \\
\hline unknown & -8.638 & -7.406 & 0.0347 & 0.4977 & -8.246 & -7.146 & 0.0591 & 0.4631 \\
\hline unknown & 8.417 & 8.385 & 0.8954 & 0.9576 & 8.584 & 8.474 & 0.5658 & 0.8256 \\
\hline unknown & -7.245 & -6.199 & 0.0274 & 0.4977 & -6.630 & -5.650 & 0.0278 & 0.4440 \\
\hline unknown & -9.398 & -7.606 & 0.0285 & 0.4977 & -9.117 & -7.875 & 0.0437 & 0.4440 \\
\hline unknown & 9.194 & 9.275 & 0.5251 & 0.8319 & 9.145 & 9.119 & 0.8622 & 0.9364 \\
\hline unknown & -9.158 & -8.444 & 0.3415 & 0.7194 & -9.037 & -9.141 & 0.7839 & 0.9094 \\
\hline unknown & -5.126 & -4.289 & 0.0575 & 0.4977 & -4.425 & -3.482 & 0.0317 & 0.4440 \\
\hline unknown & 8.648 & 9.201 & 0.0411 & 0.4977 & 8.469 & 8.478 & 0.9729 & 0.9902 \\
\hline unknown & -7.510 & -6.337 & 0.0460 & 0.4977 & -6.497 & -5.294 & 0.0392 & 0.4440 \\
\hline unknown & -5.846 & -5.572 & 0.3849 & 0.7475 & -5.510 & -5.069 & 0.0346 & 0.4440 \\
\hline unknown & -4.458 & -3.681 & 0.0438 & 0.4977 & -4.114 & -3.738 & 0.0377 & 0.4440 \\
\hline unknown & -6.731 & -5.762 & 0.0318 & 0.4977 & -6.213 & -5.834 & 0.1380 & 0.5638 \\
\hline unknown & 9.515 & 9.909 & 0.1247 & 0.5506 & 9.744 & 9.841 & 0.4394 & 0.7766 \\
\hline unknown & 7.789 & 7.852 & 0.8921 & 0.9564 & 8.719 & 8.613 & 0.8188 & 0.9258 \\
\hline unknown & 11.209 & 11.482 & 0.2327 & 0.6207 & 11.520 & 10.865 & 0.0484 & 0.4558 \\
\hline unknown & 12.689 & 12.971 & 0.0576 & 0.4977 & 13.036 & 12.603 & 0.1234 & 0.5532 \\
\hline unknown & -8.302 & -9.477 & 0.2626 & 0.6445 & -8.573 & -9.340 & 0.3850 & 0.7540 \\
\hline unknown & -6.835 & -8.052 & 0.3734 & 0.7407 & -6.753 & -7.912 & 0.1637 & 0.5944 \\
\hline unknown & 9.244 & 9.665 & 0.2237 & 0.6171 & 9.675 & 9.397 & 0.3631 & 0.7407 \\
\hline unknown & 10.337 & 10.240 & 0.7778 & 0.9091 & 11.080 & 10.874 & 0.6157 & 0.8363 \\
\hline unknown & 10.783 & 11.085 & 0.1762 & 0.5904 & 11.079 & 10.730 & 0.1991 & 0.6305 \\
\hline unknown & 9.237 & 9.154 & 0.7488 & 0.9005 & 8.157 & 8.204 & 0.9134 & 0.9609 \\
\hline unknown & 8.182 & 8.086 & 0.8480 & 0.9356 & 8.979 & 8.979 & 0.9995 & 0.9997 \\
\hline unknown & 6.795 & 7.259 & 0.2846 & 0.6648 & 7.292 & 6.971 & 0.3649 & 0.7407 \\
\hline unknown & -8.050 & -8.554 & 0.3997 & 0.7599 & -8.180 & -8.306 & 0.7401 & 0.8948 \\
\hline unknown & -6.617 & -7.505 & 0.5517 & 0.8347 & -6.150 & -6.603 & 0.6210 & 0.8363 \\
\hline unknown & -7.829 & -7.748 & 0.7902 & 0.9100 & -7.906 & -7.804 & 0.5624 & 0.8256 \\
\hline unknown & 9.208 & 9.450 & 0.3870 & 0.7497 & 9.716 & 9.653 & 0.7596 & 0.9020 \\
\hline unknown & 10.909 & 11.187 & 0.2939 & 0.6726 & 11.185 & 11.133 & 0.8481 & 0.9294 \\
\hline unknown & 8.752 & 8.998 & 0.3602 & 0.7286 & 9.053 & 8.746 & 0.1178 & 0.5479 \\
\hline unknown & -6.733 & -5.207 & 0.0239 & 0.4977 & -6.248 & -5.122 & 0.0092 & 0.4440 \\
\hline unknown & -8.299 & -6.995 & 0.0289 & 0.4977 & -7.917 & -6.947 & 0.0206 & 0.4440 \\
\hline unknown & -8.865 & -7.517 & 0.0351 & 0.4977 & -8.288 & -7.443 & 0.0215 & 0.4440 \\
\hline unknown & 9.542 & 9.580 & 0.8886 & 0.9542 & 9.889 & 9.780 & 0.6217 & 0.8363 \\
\hline unknown & -9.215 & -7.482 & 0.0126 & 0.4977 & -8.946 & -7.510 & 0.0121 & 0.4440 \\
\hline unknown & 7.488 & 7.810 & 0.4084 & 0.7660 & 7.230 & 7.361 & 0.7443 & 0.8975 \\
\hline unknown & -4.072 & -2.745 & 0.0128 & 0.4977 & -2.793 & -2.212 & 0.0791 & 0.5026 \\
\hline unknown & -8.500 & -7.044 & 0.0244 & 0.4977 & -8.694 & -7.540 & 0.0360 & 0.4440 \\
\hline unknown & 10.322 & 10.447 & 0.2003 & 0.6008 & 10.363 & 10.283 & 0.4026 & 0.7702 \\
\hline unknown & -6.523 & -5.077 & 0.0203 & 0.4977 & -5.413 & -4.904 & 0.2757 & 0.6923 \\
\hline unknown & -7.424 & -7.285 & 0.6746 & 0.8727 & -7.565 & -7.058 & 0.0934 & 0.5296 \\
\hline unknown & -7.758 & -8.277 & 0.7424 & 0.8999 & -7.151 & -7.409 & 0.7788 & 0.9094 \\
\hline
\end{tabular}




\begin{tabular}{|c|c|c|c|c|c|c|c|c|}
\hline unknown & -7.863 & -8.909 & 0.5321 & 0.8341 & -7.377 & -7.814 & 0.5662 & 0.8256 \\
\hline unknown & -3.222 & -2.364 & 0.0560 & 0.4977 & -2.378 & -1.774 & 0.0065 & 0.4440 \\
\hline unknown & -5.262 & -5.047 & 0.4992 & 0.8203 & -4.819 & -4.642 & 0.2359 & 0.6658 \\
\hline unknown & -5.080 & -5.162 & 0.6595 & 0.8635 & -5.265 & -5.021 & 0.2365 & 0.6662 \\
\hline unknown & -8.042 & -7.957 & 0.6930 & 0.8787 & -7.749 & -7.655 & 0.6365 & 0.8408 \\
\hline unknown & -7.508 & -6.728 & 0.0308 & 0.4977 & -7.797 & -7.422 & 0.1368 & 0.5638 \\
\hline unknown & -7.218 & -6.656 & 0.0828 & 0.5174 & -7.073 & -6.837 & 0.1231 & 0.5532 \\
\hline unknown & 9.220 & 9.603 & 0.0823 & 0.5174 & 7.991 & 7.591 & 0.4888 & 0.7897 \\
\hline unknown & 11.581 & 11.880 & 0.2490 & 0.6362 & 10.271 & 10.614 & 0.3823 & 0.7540 \\
\hline unknown & -8.205 & -8.690 & 0.6911 & 0.8787 & -8.433 & -8.752 & 0.6849 & 0.8653 \\
\hline unknown & 7.413 & 7.884 & 0.0916 & 0.5232 & 7.817 & 7.806 & 0.9148 & 0.9609 \\
\hline unknown & 8.445 & 8.376 & 0.8185 & 0.9172 & 8.822 & 8.402 & 0.1398 & 0.5669 \\
\hline unknown & 9.268 & 9.107 & 0.5291 & 0.8321 & 7.942 & 8.254 & 0.4997 & 0.7924 \\
\hline unknown & -8.457 & -7.487 & 0.0805 & 0.5174 & -8.099 & -7.187 & 0.0572 & 0.4631 \\
\hline unknown & -7.471 & -6.455 & 0.0882 & 0.5174 & -7.629 & -6.612 & 0.0151 & 0.4440 \\
\hline unknown & -8.292 & -6.641 & 0.0447 & 0.4977 & -8.563 & -7.341 & 0.0053 & 0.4440 \\
\hline unknown & 8.462 & 8.558 & 0.6900 & 0.8787 & 8.404 & 8.380 & 0.8828 & 0.9449 \\
\hline unknown & 8.352 & 8.423 & 0.6383 & 0.8542 & 8.864 & 8.525 & 0.0530 & 0.4558 \\
\hline unknown & -6.740 & -5.245 & 0.0170 & 0.4977 & -6.550 & -5.477 & 0.0101 & 0.4440 \\
\hline unknown & -5.944 & -4.382 & 0.0082 & 0.4977 & -5.924 & -4.887 & 0.0045 & 0.4440 \\
\hline unknown & -6.916 & -5.610 & 0.0631 & 0.4993 & -6.240 & -5.282 & 0.0430 & 0.4440 \\
\hline unknown & 9.161 & 9.601 & 0.0220 & 0.4977 & 9.627 & 9.481 & 0.5840 & 0.8359 \\
\hline unknown & 10.218 & 10.195 & 0.9343 & 0.9754 & 10.416 & 10.255 & 0.4218 & 0.7766 \\
\hline unknown & 6.828 & 7.464 & 0.0437 & 0.4977 & 6.907 & 6.888 & 0.9191 & 0.9627 \\
\hline unknown & -8.567 & -6.497 & 0.0093 & 0.4977 & -8.480 & -7.249 & 0.0082 & 0.4440 \\
\hline unknown & 8.062 & 7.929 & 0.7273 & 0.8936 & 8.226 & 8.108 & 0.7001 & 0.8740 \\
\hline unknown & 7.981 & 8.529 & 0.0320 & 0.4977 & 8.480 & 8.041 & 0.3335 & 0.7310 \\
\hline unknown & -7.455 & -7.771 & 0.8512 & 0.9366 & -6.523 & -6.648 & 0.8767 & 0.9406 \\
\hline unknown & -10.314 & -10.423 & 0.9393 & 0.9756 & -8.823 & -9.171 & 0.6964 & 0.8732 \\
\hline unknown & -3.395 & -2.006 & 0.0070 & 0.4977 & -2.431 & -1.666 & 0.0091 & 0.4440 \\
\hline unknown & -8.134 & -6.431 & 0.0278 & 0.4977 & -7.847 & -6.708 & 0.0691 & 0.4920 \\
\hline unknown & 7.550 & 7.886 & 0.0660 & 0.5051 & 7.252 & 7.406 & 0.5175 & 0.7975 \\
\hline unknown & 11.089 & 11.206 & 0.3727 & 0.7407 & 10.924 & 10.963 & 0.8048 & 0.9201 \\
\hline unknown & -5.233 & -4.714 & 0.1562 & 0.5769 & -4.917 & -4.423 & 0.0271 & 0.4440 \\
\hline unknown & 8.773 & 8.963 & 0.2700 & 0.6524 & 8.440 & 8.559 & 0.5424 & 0.8057 \\
\hline unknown & -6.747 & -6.710 & 0.8799 & 0.9486 & -6.898 & -6.544 & 0.2333 & 0.6658 \\
\hline unknown & -8.390 & -8.892 & 0.7410 & 0.8999 & -8.480 & -8.998 & 0.5697 & 0.8262 \\
\hline unknown & -2.974 & -1.989 & 0.0361 & 0.4977 & -2.087 & -1.294 & 0.0247 & 0.4440 \\
\hline unknown & -7.038 & -6.453 & 0.0794 & 0.5174 & -6.699 & -6.214 & 0.0228 & 0.4440 \\
\hline unknown & -6.454 & -5.364 & 0.0211 & 0.4977 & -6.540 & -6.293 & 0.3330 & 0.7310 \\
\hline unknown & 9.072 & 9.434 & 0.1108 & 0.5435 & 8.023 & 7.641 & 0.3641 & 0.7407 \\
\hline unknown & -5.531 & -5.784 & 0.3692 & 0.7367 & -4.825 & -4.788 & 0.8175 & 0.9252 \\
\hline unknown & -9.018 & -7.747 & 0.1094 & 0.5435 & -9.725 & -8.112 & 0.0003 & 0.2240 \\
\hline unknown & -6.228 & -5.657 & 0.1708 & 0.5892 & -6.583 & -6.528 & 0.8989 & 0.9541 \\
\hline unknown & 9.573 & 9.938 & 0.1455 & 0.5602 & 8.955 & 9.041 & 0.7624 & 0.9029 \\
\hline unknown & 7.276 & 7.696 & 0.2235 & 0.6171 & 6.618 & 6.514 & 0.7370 & 0.8944 \\
\hline unknown & -7.957 & -6.840 & 0.1192 & 0.5435 & -8.077 & -7.139 & 0.0355 & 0.4440 \\
\hline unknown & -7.757 & -6.604 & 0.1071 & 0.5435 & -7.663 & -6.724 & 0.0446 & 0.4440 \\
\hline unknown & 8.630 & 8.755 & 0.7139 & 0.8917 & 8.825 & 8.302 & 0.1562 & 0.5918 \\
\hline unknown & -7.263 & -5.762 & 0.0296 & 0.4977 & -7.541 & -6.455 & 0.0046 & 0.4440 \\
\hline unknown & -6.031 & -5.016 & 0.1441 & 0.5602 & -5.853 & -5.144 & 0.1059 & 0.5479 \\
\hline unknown & -6.045 & -5.124 & 0.1034 & 0.5435 & -5.920 & -5.439 & 0.3362 & 0.7310 \\
\hline unknown & -5.147 & -3.766 & 0.0209 & 0.4977 & -4.638 & -3.844 & 0.0178 & 0.4440 \\
\hline unknown & -6.415 & -7.337 & 0.2295 & 0.6180 & -6.470 & -6.756 & 0.6938 & 0.8724 \\
\hline unknown & -9.816 & -9.154 & 0.4718 & 0.8059 & -8.919 & -9.658 & 0.1948 & 0.6302 \\
\hline unknown & -8.691 & -9.806 & 0.2113 & 0.6124 & -9.000 & -9.303 & 0.7323 & 0.8944 \\
\hline unknown & -7.485 & -6.569 & 0.0100 & 0.4977 & -7.180 & -6.760 & 0.1695 & 0.5944 \\
\hline
\end{tabular}




\begin{tabular}{|c|c|c|c|c|c|c|c|c|}
\hline unknown & 8.816 & 9.122 & 0.1221 & 0.5464 & 8.718 & 8.753 & 0.8255 & 0.9282 \\
\hline unknown & -5.247 & -4.691 & 0.1823 & 0.5904 & -4.709 & -4.234 & 0.0601 & 0.4631 \\
\hline unknown & -8.485 & -7.047 & 0.0592 & 0.4977 & -7.359 & -7.876 & 0.4767 & 0.7840 \\
\hline unknown & -8.278 & -8.678 & 0.7867 & 0.9093 & -8.055 & -8.595 & 0.4524 & 0.7820 \\
\hline unknown & -6.230 & -5.639 & 0.0333 & 0.4977 & -6.166 & -5.638 & 0.1564 & 0.5918 \\
\hline unknown & -5.323 & -4.692 & 0.0784 & 0.5174 & -4.584 & -4.270 & 0.1605 & 0.5933 \\
\hline unknown & -6.467 & -6.307 & 0.4089 & 0.7660 & -6.308 & -6.065 & 0.2441 & 0.6774 \\
\hline unknown & -5.630 & -5.487 & 0.5182 & 0.8297 & -5.858 & -5.728 & 0.2199 & 0.6419 \\
\hline unknown & -8.483 & -9.077 & 0.6506 & 0.8622 & -7.856 & -8.002 & 0.8575 & 0.9336 \\
\hline unknown & -9.294 & -9.607 & 0.8065 & 0.9142 & -7.808 & -8.412 & 0.5732 & 0.8282 \\
\hline unknown & -5.269 & -5.045 & 0.5222 & 0.8300 & -4.562 & -4.375 & 0.3125 & 0.7157 \\
\hline unknown & -7.613 & -6.995 & 0.0766 & 0.5174 & -7.083 & -6.537 & 0.0211 & 0.4440 \\
\hline unknown & -3.971 & -4.583 & 0.0129 & 0.4977 & -3.975 & -4.011 & 0.8757 & 0.9406 \\
\hline unknown & 7.747 & 8.095 & 0.2341 & 0.6212 & 7.707 & 7.586 & 0.6090 & 0.8363 \\
\hline unknown & -7.571 & -7.519 & 0.8171 & 0.9172 & -8.092 & -7.601 & 0.1037 & 0.5450 \\
\hline unknown & -8.070 & -7.680 & 0.0609 & 0.4984 & -8.164 & -7.765 & 0.0138 & 0.4440 \\
\hline unknown & -5.965 & -6.855 & 0.0195 & 0.4977 & -5.971 & -6.121 & 0.6483 & 0.8471 \\
\hline unknown & -6.674 & -6.477 & 0.5038 & 0.8231 & -7.572 & -7.479 & 0.8148 & 0.9244 \\
\hline unknown & -5.603 & -4.970 & 0.2407 & 0.6276 & -7.130 & -6.934 & 0.7397 & 0.8948 \\
\hline unknown & -7.572 & -7.365 & 0.2186 & 0.6162 & -7.476 & -7.253 & 0.0323 & 0.4440 \\
\hline unknown & 7.515 & 7.992 & 0.1404 & 0.5584 & 7.461 & 7.324 & 0.6178 & 0.8363 \\
\hline unknown & 9.064 & 9.294 & 0.4839 & 0.8099 & 7.000 & 7.568 & 0.3138 & 0.7163 \\
\hline unknown & -6.095 & -4.696 & 0.1060 & 0.5435 & -6.123 & -4.743 & 0.0106 & 0.4440 \\
\hline unknown & 10.651 & 11.119 & 0.0463 & 0.4977 & 10.928 & 10.807 & 0.6550 & 0.8510 \\
\hline unknown & 6.294 & 6.451 & 0.6911 & 0.8787 & 4.181 & 5.403 & 0.1353 & 0.5638 \\
\hline unknown & -4.592 & -3.349 & 0.0787 & 0.5174 & -4.656 & -3.521 & 0.0670 & 0.4876 \\
\hline unknown & -9.024 & -7.403 & 0.0163 & 0.4977 & -10.076 & -9.126 & 0.1111 & 0.5479 \\
\hline unknown & 12.730 & 13.027 & 0.1619 & 0.5769 & 13.235 & 13.077 & 0.4693 & 0.7838 \\
\hline unknown & -6.771 & -5.339 & 0.0890 & 0.5195 & -6.837 & -5.556 & 0.0586 & 0.4631 \\
\hline unknown & -8.670 & -7.465 & 0.0311 & 0.4977 & -8.197 & -7.168 & 0.0600 & 0.4631 \\
\hline unknown & -5.815 & -4.625 & 0.0225 & 0.4977 & -6.776 & -5.920 & 0.0117 & 0.4440 \\
\hline unknown & -8.525 & -6.843 & 0.0094 & 0.4977 & -9.878 & -8.918 & 0.0499 & 0.4558 \\
\hline unknown & -8.695 & -8.189 & 0.4518 & 0.7960 & -9.246 & -8.325 & 0.1861 & 0.6161 \\
\hline unknown & -4.338 & -3.783 & 0.2073 & 0.6047 & -3.329 & -2.621 & 0.1405 & 0.5673 \\
\hline unknown & -6.135 & -5.780 & 0.4374 & 0.7863 & -4.859 & -4.510 & 0.4642 & 0.7838 \\
\hline unknown & -7.789 & -6.297 & 0.0187 & 0.4977 & -6.795 & -5.769 & 0.0041 & 0.4440 \\
\hline unknown & -6.644 & -5.495 & 0.0134 & 0.4977 & -6.033 & -5.558 & 0.1000 & 0.5380 \\
\hline unknown & 9.494 & 9.689 & 0.3076 & 0.6837 & 8.404 & 8.401 & 0.9947 & 0.9990 \\
\hline unknown & -6.401 & -5.654 & 0.2177 & 0.6150 & -5.418 & -4.698 & 0.1671 & 0.5944 \\
\hline unknown & -9.173 & -9.674 & 0.6339 & 0.8542 & -8.559 & -9.039 & 0.4696 & 0.7838 \\
\hline unknown & -8.281 & -8.938 & 0.6355 & 0.8542 & -7.968 & -8.480 & 0.5267 & 0.8034 \\
\hline unknown & -8.112 & -8.811 & 0.6450 & 0.8589 & -8.257 & -9.199 & 0.2581 & 0.6791 \\
\hline unknown & -2.413 & -2.386 & 0.9348 & 0.9754 & -1.005 & -0.885 & 0.6703 & 0.8591 \\
\hline unknown & -6.003 & -5.310 & 0.0305 & 0.4977 & -5.271 & -4.826 & 0.0839 & 0.5105 \\
\hline unknown & -6.386 & -5.224 & 0.0404 & 0.4977 & -5.694 & -4.969 & 0.0524 & 0.4558 \\
\hline unknown & -4.630 & -4.952 & 0.2316 & 0.6207 & -3.782 & -3.670 & 0.7699 & 0.9073 \\
\hline unknown & -8.774 & -7.854 & 0.0281 & 0.4977 & -7.848 & -7.276 & 0.0793 & 0.5026 \\
\hline unknown & -9.223 & -7.770 & 0.0321 & 0.4977 & -8.263 & -7.510 & 0.0808 & 0.5026 \\
\hline unknown & -8.165 & -8.851 & 0.5502 & 0.8347 & -7.866 & -8.290 & 0.6209 & 0.8363 \\
\hline unknown & -8.272 & -8.667 & 0.7476 & 0.9005 & -7.590 & -7.860 & 0.7354 & 0.8944 \\
\hline unknown & -8.017 & -8.730 & 0.5876 & 0.8433 & -7.469 & -7.624 & 0.8695 & 0.9390 \\
\hline unknown & -9.011 & -9.108 & 0.9349 & 0.9754 & -7.571 & -8.029 & 0.6569 & 0.8519 \\
\hline unknown & -3.709 & -3.563 & 0.5638 & 0.8347 & -3.000 & -2.572 & 0.3505 & 0.7373 \\
\hline unknown & -4.457 & -4.868 & 0.0504 & 0.4977 & -5.006 & -4.941 & 0.8150 & 0.9244 \\
\hline unknown & -5.542 & -5.336 & 0.5485 & 0.8347 & -5.127 & -5.054 & 0.8225 & 0.9277 \\
\hline unknown & -2.952 & -2.848 & 0.8210 & 0.9172 & -1.810 & -1.626 & 0.5109 & 0.7935 \\
\hline unknown & -6.620 & -7.302 & 0.0326 & 0.4977 & -7.421 & -7.467 & 0.8979 & 0.9541 \\
\hline
\end{tabular}




\begin{tabular}{|c|c|c|c|c|c|c|c|c|}
\hline unknown & -6.655 & -5.058 & 0.0865 & 0.5174 & -7.208 & -5.877 & 0.0806 & 0.5026 \\
\hline unknown & -1.530 & -1.682 & 0.3186 & 0.6958 & -1.322 & -1.249 & 0.3844 & 0.7540 \\
\hline unknown & 5.272 & 6.110 & 0.1471 & 0.5621 & 6.798 & 5.452 & 0.1860 & 0.6161 \\
\hline unknown & -4.832 & -3.633 & 0.1446 & 0.5602 & -4.843 & -3.780 & 0.0934 & 0.5296 \\
\hline unknown & -5.676 & -4.091 & 0.0596 & 0.4977 & -6.067 & -4.907 & 0.0200 & 0.4440 \\
\hline unknown & 6.969 & 7.055 & 0.7022 & 0.8843 & 6.293 & 6.141 & 0.5009 & 0.7924 \\
\hline unknown & 9.067 & 9.589 & 0.0197 & 0.4977 & 9.108 & 9.178 & 0.7072 & 0.8771 \\
\hline unknown & 6.497 & 6.687 & 0.4462 & 0.7918 & 6.772 & 6.510 & 0.1119 & 0.5479 \\
\hline unknown & -7.105 & -5.573 & 0.1576 & 0.5769 & -7.186 & -5.968 & 0.1192 & 0.5479 \\
\hline unknown & -8.107 & -6.189 & 0.0544 & 0.4977 & -8.839 & -7.368 & 0.0415 & 0.4440 \\
\hline unknown & -7.186 & -6.364 & 0.0928 & 0.5277 & -7.488 & -7.106 & 0.4343 & 0.7766 \\
\hline unknown & -5.124 & -4.088 & 0.1152 & 0.5435 & -5.740 & -4.969 & 0.1364 & 0.5638 \\
\hline unknown & 10.433 & 10.775 & 0.0476 & 0.4977 & 10.933 & 10.501 & 0.1517 & 0.5918 \\
\hline unknown & 10.591 & 10.833 & 0.1702 & 0.5888 & 10.606 & 10.248 & 0.2248 & 0.6528 \\
\hline unknown & 8.852 & 9.216 & 0.0675 & 0.5054 & 7.837 & 8.037 & 0.5972 & 0.8363 \\
\hline unknown & 8.276 & 8.318 & 0.7978 & 0.9122 & 6.823 & 7.456 & 0.2958 & 0.7057 \\
\hline unknown & -6.020 & -4.983 & 0.1851 & 0.5904 & -5.807 & -5.090 & 0.2559 & 0.6789 \\
\hline unknown & -7.632 & -6.106 & 0.1018 & 0.5435 & -8.413 & -7.453 & 0.1187 & 0.5479 \\
\hline unknown & -7.930 & -8.391 & 0.5396 & 0.8347 & -8.448 & -8.787 & 0.6101 & 0.8363 \\
\hline unknown & -8.869 & -7.943 & 0.2276 & 0.6171 & -7.957 & -8.528 & 0.2788 & 0.6923 \\
\hline unknown & -8.695 & -9.241 & 0.6347 & 0.8542 & -8.312 & -9.059 & 0.4037 & 0.7702 \\
\hline unknown & -9.478 & -9.890 & 0.7576 & 0.9027 & -8.626 & -9.155 & 0.4897 & 0.7897 \\
\hline unknown & -7.030 & -6.039 & 0.0594 & 0.4977 & -6.708 & -6.095 & 0.1698 & 0.5944 \\
\hline unknown & -7.575 & -7.173 & 0.1202 & 0.5435 & -6.866 & -6.441 & 0.1424 & 0.5712 \\
\hline unknown & -6.394 & -5.560 & 0.1787 & 0.5904 & -5.565 & -5.135 & 0.4145 & 0.7763 \\
\hline unknown & -7.089 & -5.949 & 0.0353 & 0.4977 & -5.913 & -5.519 & 0.3444 & 0.7373 \\
\hline unknown & -8.979 & -9.567 & 0.6663 & 0.8681 & -8.464 & -9.638 & 0.1641 & 0.5944 \\
\hline unknown & -8.690 & -9.263 & 0.6930 & 0.8787 & -8.067 & -8.958 & 0.3641 & 0.7407 \\
\hline unknown & -9.328 & -9.704 & 0.7290 & 0.8936 & -7.958 & -8.449 & 0.6285 & 0.8380 \\
\hline unknown & -4.441 & -3.884 & 0.1414 & 0.5593 & -3.372 & -3.226 & 0.7196 & 0.8871 \\
\hline unknown & -6.580 & -6.435 & 0.6296 & 0.8521 & -6.780 & -6.578 & 0.5325 & 0.8034 \\
\hline unknown & -4.711 & -5.057 & 0.1159 & 0.5435 & -4.608 & -4.531 & 0.5414 & 0.8057 \\
\hline unknown & -6.579 & -5.861 & 0.1403 & 0.5584 & -5.407 & -5.032 & 0.3943 & 0.7608 \\
\hline unknown & 8.280 & 8.984 & 0.0389 & 0.4977 & 8.927 & 9.028 & 0.7186 & 0.8867 \\
\hline unknown & -7.083 & -6.186 & 0.1177 & 0.5435 & -6.718 & -6.264 & 0.4075 & 0.7728 \\
\hline unknown & -6.960 & -7.398 & 0.1025 & 0.5435 & -6.853 & -6.852 & 0.9965 & 0.9990 \\
\hline unknown & -9.447 & -10.366 & 0.3611 & 0.7293 & -8.412 & -9.333 & 0.2530 & 0.6776 \\
\hline unknown & -8.889 & -9.399 & 0.7027 & 0.8843 & -7.812 & -8.219 & 0.6275 & 0.8380 \\
\hline unknown & -4.167 & -4.178 & 0.9649 & 0.9827 & -4.087 & -3.935 & 0.5282 & 0.8034 \\
\hline unknown & -2.438 & -1.920 & 0.2433 & 0.6296 & -2.202 & -1.955 & 0.3514 & 0.7373 \\
\hline unknown & 10.514 & 9.806 & 0.0393 & 0.4977 & 10.741 & 10.304 & 0.0801 & 0.5026 \\
\hline unknown & -6.329 & -6.439 & 0.7273 & 0.8936 & -6.740 & -6.727 & 0.9674 & 0.9879 \\
\hline unknown & -6.310 & -6.308 & 0.9949 & 0.9972 & -6.187 & -6.061 & 0.7266 & 0.8912 \\
\hline unknown & -4.871 & -4.658 & 0.4557 & 0.7971 & -4.848 & -4.756 & 0.5280 & 0.8034 \\
\hline unknown & 8.400 & 7.484 & 0.0587 & 0.4977 & 8.689 & 8.207 & 0.1301 & 0.5621 \\
\hline unknown & -4.841 & -2.950 & 0.0766 & 0.5174 & -5.793 & -3.729 & 0.0503 & 0.4558 \\
\hline unknown & -6.358 & -5.955 & 0.2344 & 0.6212 & -6.088 & -6.064 & 0.9617 & 0.9840 \\
\hline unknown & -5.812 & -5.512 & 0.4136 & 0.7674 & -7.131 & -6.824 & 0.3409 & 0.7362 \\
\hline unknown & 7.647 & 7.647 & 0.9992 & 0.9999 & 7.625 & 7.587 & 0.8368 & 0.9282 \\
\hline unknown & -6.879 & -5.138 & 0.0989 & 0.5435 & -8.237 & -5.635 & 0.0496 & 0.4558 \\
\hline unknown & -6.680 & -6.607 & 0.8245 & 0.9204 & -6.377 & -6.001 & 0.1164 & 0.5479 \\
\hline unknown & -10.541 & -10.129 & 0.4783 & 0.8099 & -9.325 & -8.578 & 0.1547 & 0.5918 \\
\hline unknown & -3.444 & -1.500 & 0.0404 & 0.4977 & -4.008 & -2.256 & 0.0565 & 0.4631 \\
\hline unknown & 8.642 & 8.909 & 0.1954 & 0.5980 & 7.692 & 7.399 & 0.4621 & 0.7838 \\
\hline unknown & -5.671 & -4.192 & 0.0720 & 0.5159 & -6.335 & -4.869 & 0.0761 & 0.4986 \\
\hline unknown & -8.075 & -8.672 & 0.4005 & 0.7604 & -8.223 & -8.405 & 0.6953 & 0.8726 \\
\hline unknown & -8.014 & -7.496 & 0.3853 & 0.7475 & -7.214 & -6.163 & 0.1127 & 0.5479 \\
\hline
\end{tabular}




\begin{tabular}{|c|c|c|c|c|c|c|c|c|}
\hline unknown & -4.333 & -3.728 & 0.3844 & 0.7475 & -3.653 & -2.968 & 0.2157 & 0.6419 \\
\hline unknown & -7.233 & -6.355 & 0.1158 & 0.5435 & -7.066 & -6.669 & 0.3688 & 0.7429 \\
\hline unknown & -5.953 & -4.886 & 0.1627 & 0.5769 & -6.230 & -5.088 & 0.1278 & 0.5583 \\
\hline unknown & 11.244 & 11.520 & 0.1695 & 0.5878 & 10.125 & 10.281 & 0.7153 & 0.8843 \\
\hline unknown & 6.796 & 6.698 & 0.5147 & 0.8274 & 6.919 & 6.676 & 0.3288 & 0.7305 \\
\hline unknown & 7.149 & 7.129 & 0.9041 & 0.9615 & 5.645 & 6.474 & 0.1263 & 0.5575 \\
\hline unknown & -6.630 & -5.571 & 0.1941 & 0.5980 & -5.672 & -4.815 & 0.1780 & 0.6073 \\
\hline unknown & -7.151 & -6.693 & 0.3062 & 0.6837 & -6.471 & -5.557 & 0.1046 & 0.5450 \\
\hline unknown & -4.743 & -3.951 & 0.1436 & 0.5602 & -4.182 & -3.576 & 0.2806 & 0.6923 \\
\hline unknown & -8.586 & -7.210 & 0.0593 & 0.4977 & -9.662 & -8.464 & 0.0978 & 0.5348 \\
\hline unknown & 8.455 & 8.080 & 0.1659 & 0.5796 & 7.494 & 7.931 & 0.3184 & 0.7192 \\
\hline unknown & -4.400 & -4.387 & 0.9711 & 0.9833 & -3.624 & -3.247 & 0.4522 & 0.7820 \\
\hline unknown & -7.643 & -8.196 & 0.4404 & 0.7899 & -7.980 & -8.300 & 0.6460 & 0.8451 \\
\hline unknown & -5.158 & -5.209 & 0.8789 & 0.9486 & -4.579 & -4.106 & 0.0508 & 0.4558 \\
\hline unknown & -4.037 & -3.671 & 0.1471 & 0.5621 & -3.640 & -3.146 & 0.3296 & 0.7310 \\
\hline unknown & -6.065 & -5.475 & 0.0839 & 0.5174 & -5.693 & -5.100 & 0.0657 & 0.4834 \\
\hline unknown & -7.384 & -7.484 & 0.7974 & 0.9122 & -6.683 & -6.198 & 0.0577 & 0.4631 \\
\hline unknown & -4.765 & -3.888 & 0.0826 & 0.5174 & -3.625 & -3.226 & 0.3376 & 0.7317 \\
\hline unknown & -9.774 & -10.452 & 0.4262 & 0.7757 & -9.717 & -9.610 & 0.9044 & 0.9571 \\
\hline unknown & -8.930 & -9.228 & 0.8019 & 0.9124 & -8.626 & -8.932 & 0.6793 & 0.8618 \\
\hline unknown & -1.665 & -1.115 & 0.1863 & 0.5904 & -0.451 & -0.150 & 0.4377 & 0.7766 \\
\hline unknown & -4.768 & -4.735 & 0.8613 & 0.9397 & -4.245 & -3.945 & 0.3154 & 0.7175 \\
\hline unknown & -1.646 & -1.151 & 0.2485 & 0.6362 & -1.210 & -0.931 & 0.2029 & 0.6332 \\
\hline unknown & -4.045 & -4.063 & 0.9363 & 0.9756 & -3.648 & -3.572 & 0.7844 & 0.9094 \\
\hline unknown & -8.217 & -7.862 & 0.0258 & 0.4977 & -7.727 & -7.644 & 0.4776 & 0.7846 \\
\hline unknown & -9.400 & -9.709 & 0.4916 & 0.8142 & -6.830 & -7.216 & 0.4380 & 0.7766 \\
\hline unknown & -8.454 & -9.029 & 0.6428 & 0.8569 & -7.966 & -8.525 & 0.4075 & 0.7728 \\
\hline unknown & -8.389 & -9.264 & 0.5369 & 0.8347 & -6.505 & -6.973 & 0.6216 & 0.8363 \\
\hline unknown & -2.207 & -2.319 & 0.6773 & 0.8730 & -1.858 & -2.330 & 0.0744 & 0.4957 \\
\hline unknown & 7.779 & 7.855 & 0.6139 & 0.8511 & 7.710 & 7.635 & 0.6118 & 0.8363 \\
\hline unknown & -7.405 & -5.722 & 0.1396 & 0.5584 & -7.625 & -6.318 & 0.2518 & 0.6776 \\
\hline unknown & -8.755 & -8.271 & 0.4166 & 0.7691 & -6.088 & -6.193 & 0.8515 & 0.9294 \\
\hline unknown & -3.874 & -3.663 & 0.4763 & 0.8080 & -3.067 & -2.830 & 0.4555 & 0.7829 \\
\hline unknown & -3.238 & -3.435 & 0.2914 & 0.6709 & -3.271 & -3.300 & 0.8773 & 0.9406 \\
\hline unknown & 5.059 & 5.602 & 0.3538 & 0.7286 & 6.427 & 4.441 & 0.1439 & 0.5757 \\
\hline unknown & -5.785 & -5.434 & 0.3825 & 0.7475 & -5.013 & -4.945 & 0.7513 & 0.9011 \\
\hline unknown & -10.861 & -10.618 & 0.5025 & 0.8229 & -8.397 & -8.653 & 0.6657 & 0.8573 \\
\hline unknown & -2.873 & -1.293 & 0.0736 & 0.5174 & -2.935 & -1.683 & 0.1080 & 0.5479 \\
\hline unknown & -8.699 & -7.199 & 0.1365 & 0.5584 & -8.499 & -7.399 & 0.2341 & 0.6658 \\
\hline unknown & -5.312 & -3.983 & 0.0690 & 0.5054 & -5.316 & -4.326 & 0.1593 & 0.5926 \\
\hline unknown & -7.463 & -6.473 & 0.1487 & 0.5634 & -6.797 & -6.164 & 0.4225 & 0.7766 \\
\hline unknown & 6.771 & 6.894 & 0.7593 & 0.9038 & 4.489 & 5.791 & 0.1365 & 0.5638 \\
\hline unknown & -5.883 & -5.027 & 0.2229 & 0.6171 & -6.608 & -5.573 & 0.1182 & 0.5479 \\
\hline unknown & -8.944 & -8.966 & 0.9233 & 0.9705 & -8.097 & -8.025 & 0.8603 & 0.9360 \\
\hline unknown & -4.576 & -3.828 & 0.2492 & 0.6362 & -4.132 & -3.463 & 0.1580 & 0.5926 \\
\hline unknown & -5.189 & -4.734 & 0.2977 & 0.6729 & -5.674 & -5.551 & 0.7800 & 0.9094 \\
\hline unknown & -6.785 & -5.886 & 0.2821 & 0.6628 & -6.246 & -5.481 & 0.2999 & 0.7120 \\
\hline unknown & -7.749 & -7.082 & 0.2941 & 0.6726 & -8.325 & -8.441 & 0.8392 & 0.9282 \\
\hline unknown & -8.687 & -8.515 & 0.5638 & 0.8347 & -8.963 & -8.578 & 0.2134 & 0.6419 \\
\hline unknown & -7.274 & -6.453 & 0.2709 & 0.6526 & -6.815 & -6.301 & 0.3082 & 0.7132 \\
\hline unknown & -8.619 & -9.327 & 0.6334 & 0.8542 & -8.226 & -8.692 & 0.5130 & 0.7935 \\
\hline unknown & -8.780 & -9.311 & 0.7430 & 0.8999 & -7.866 & -8.188 & 0.6743 & 0.8610 \\
\hline unknown & -8.936 & -9.368 & 0.7379 & 0.8991 & -7.321 & -8.210 & 0.3057 & 0.7132 \\
\hline unknown & -7.161 & -6.550 & 0.1898 & 0.5932 & -5.807 & -5.320 & 0.1851 & 0.6159 \\
\hline unknown & -6.952 & -6.166 & 0.1063 & 0.5435 & -7.320 & -7.276 & 0.9024 & 0.9559 \\
\hline unknown & -8.062 & -8.683 & 0.6588 & 0.8635 & -8.299 & -8.947 & 0.5212 & 0.7986 \\
\hline unknown & -8.550 & -9.292 & 0.6177 & 0.8511 & -8.300 & -9.508 & 0.1945 & 0.6302 \\
\hline
\end{tabular}




\begin{tabular}{|c|c|c|c|c|c|c|c|c|}
\hline unknown & -8.966 & -9.718 & 0.5001 & 0.8203 & -7.629 & -8.273 & 0.2567 & 0.6789 \\
\hline unknown & -9.120 & -10.080 & 0.3800 & 0.7462 & -7.676 & -8.868 & 0.1307 & 0.5621 \\
\hline unknown & -7.972 & -9.536 & 0.2648 & 0.6470 & -7.218 & -8.332 & 0.2477 & 0.6776 \\
\hline unknown & -7.207 & -7.653 & 0.7776 & 0.9091 & -5.834 & -6.206 & 0.6886 & 0.8683 \\
\hline unknown & -5.856 & -5.308 & 0.1252 & 0.5509 & -5.392 & -5.287 & 0.7378 & 0.8945 \\
\hline unknown & -5.484 & -5.250 & 0.4326 & 0.7798 & -5.201 & -5.148 & 0.6008 & 0.8363 \\
\hline unknown & -9.375 & -9.958 & 0.6229 & 0.8511 & -8.250 & -8.602 & 0.7353 & 0.8944 \\
\hline unknown & 9.776 & 9.930 & 0.4726 & 0.8059 & 10.256 & 10.188 & 0.6211 & 0.8363 \\
\hline unknown & -6.649 & -7.434 & 0.5845 & 0.8433 & -5.594 & -6.630 & 0.2811 & 0.6923 \\
\hline unknown & -7.039 & -7.730 & 0.6570 & 0.8635 & -5.745 & -7.077 & 0.2176 & 0.6419 \\
\hline unknown & -3.614 & -3.311 & 0.4822 & 0.8099 & -3.915 & -3.601 & 0.1555 & 0.5918 \\
\hline unknown & 9.685 & 10.287 & 0.0566 & 0.4977 & 9.807 & 9.774 & 0.8445 & 0.9290 \\
\hline unknown & -8.960 & -9.868 & 0.5152 & 0.8274 & -7.918 & -9.032 & 0.2683 & 0.6884 \\
\hline unknown & -9.249 & -10.011 & 0.5537 & 0.8347 & -8.198 & -9.677 & 0.2274 & 0.6546 \\
\hline unknown & -6.064 & -5.347 & 0.1086 & 0.5435 & -6.348 & -5.915 & 0.1520 & 0.5918 \\
\hline unknown & -7.778 & -8.264 & 0.1475 & 0.5621 & -7.348 & -7.396 & 0.8711 & 0.9400 \\
\hline unknown & -7.194 & -7.235 & 0.7843 & 0.9091 & -7.320 & -7.237 & 0.6627 & 0.8552 \\
\hline unknown & 9.506 & 9.525 & 0.7665 & 0.9044 & 8.521 & 9.031 & 0.2752 & 0.6923 \\
\hline unknown & 7.128 & 7.183 & 0.3188 & 0.6958 & 6.693 & 6.810 & 0.7226 & 0.8884 \\
\hline unknown & 7.114 & 7.247 & 0.0301 & 0.4977 & 6.792 & 6.798 & 0.9878 & 0.9969 \\
\hline unknown & 6.702 & 6.872 & 0.1727 & 0.5904 & 6.924 & 6.750 & 0.0658 & 0.4834 \\
\hline unknown & -8.821 & -7.568 & 0.1855 & 0.5904 & -7.749 & -6.928 & 0.2149 & 0.6419 \\
\hline unknown & -7.115 & -6.424 & 0.0249 & 0.4977 & -7.829 & -7.454 & 0.3017 & 0.7120 \\
\hline unknown & -6.364 & -6.087 & 0.2667 & 0.6484 & -7.034 & -6.810 & 0.0454 & 0.4440 \\
\hline unknown & -8.034 & -7.330 & 0.0348 & 0.4977 & -7.421 & -7.639 & 0.5175 & 0.7975 \\
\hline unknown & -2.520 & -2.715 & 0.2866 & 0.6661 & -2.592 & -2.661 & 0.7524 & 0.9015 \\
\hline unknown & 8.037 & 8.121 & 0.0034 & 0.4977 & 7.561 & 7.785 & 0.4136 & 0.7763 \\
\hline unknown & 7.944 & 8.004 & 0.5816 & 0.8433 & 7.987 & 7.962 & 0.4252 & 0.7766 \\
\hline unknown & -4.668 & -3.678 & 0.1975 & 0.6008 & -4.256 & -2.913 & 0.0308 & 0.4440 \\
\hline unknown & -3.805 & -2.766 & 0.2148 & 0.6142 & -3.423 & -2.716 & 0.3209 & 0.7218 \\
\hline unknown & -5.607 & -4.405 & 0.0511 & 0.4977 & -5.678 & -4.609 & 0.0453 & 0.4440 \\
\hline unknown & -7.396 & -8.350 & 0.5091 & 0.8252 & -6.658 & -7.065 & 0.6711 & 0.8594 \\
\hline unknown & -12.509 & -15.462 & 0.1352 & 0.5584 & -9.273 & -9.298 & 0.9800 & 0.9928 \\
\hline unknown & -12.061 & -13.358 & 0.5193 & 0.8300 & -8.339 & -8.298 & 0.9693 & 0.9888 \\
\hline unknown & -3.682 & -3.153 & 0.3231 & 0.6973 & -3.679 & -2.956 & 0.0727 & 0.4936 \\
\hline unknown & -1.731 & -0.733 & 0.1589 & 0.5769 & -1.298 & -0.513 & 0.1705 & 0.5944 \\
\hline unknown & -0.942 & -0.030 & 0.1885 & 0.5932 & -0.664 & 0.168 & 0.0681 & 0.4901 \\
\hline unknown & -4.338 & -3.495 & 0.1653 & 0.5796 & -3.889 & -3.283 & 0.1609 & 0.5933 \\
\hline unknown & -3.530 & -2.595 & 0.2018 & 0.6009 & -3.392 & -2.467 & 0.0710 & 0.4936 \\
\hline unknown & 0.956 & 1.508 & 0.2796 & 0.6591 & 1.213 & 1.845 & 0.1144 & 0.5479 \\
\hline unknown & -5.000 & -5.044 & 0.8818 & 0.9497 & -5.103 & -4.953 & 0.6182 & 0.8363 \\
\hline unknown & -4.483 & -3.757 & 0.1280 & 0.5584 & -5.071 & -4.711 & 0.5525 & 0.8165 \\
\hline unknown & -6.255 & -5.406 & 0.0909 & 0.5227 & -7.039 & -6.778 & 0.5807 & 0.8335 \\
\hline unknown & -4.614 & -3.757 & 0.1151 & 0.5435 & -5.386 & -5.048 & 0.6026 & 0.8363 \\
\hline unknown & -8.400 & -8.663 & 0.7040 & 0.8850 & -7.950 & -8.138 & 0.4091 & 0.7728 \\
\hline unknown & -4.256 & -4.266 & 0.9726 & 0.9833 & -3.826 & -3.561 & 0.3239 & 0.7256 \\
\hline unknown & -1.595 & -1.289 & 0.5502 & 0.8347 & -1.966 & -1.042 & 0.0141 & 0.4440 \\
\hline unknown & -5.544 & -4.943 & 0.2787 & 0.6587 & -5.436 & -5.465 & 0.9457 & 0.9759 \\
\hline unknown & -6.468 & -6.439 & 0.9450 & 0.9767 & -5.885 & -5.594 & 0.4294 & 0.7766 \\
\hline unknown & -4.355 & -4.023 & 0.5002 & 0.8203 & -4.335 & -4.346 & 0.9760 & 0.9917 \\
\hline unknown & -3.422 & -2.610 & 0.1405 & 0.5584 & -3.729 & -3.626 & 0.8226 & 0.9277 \\
\hline unknown & -8.707 & -9.893 & 0.1759 & 0.5904 & -8.852 & -9.403 & 0.5873 & 0.8363 \\
\hline unknown & -7.467 & -7.769 & 0.2225 & 0.6171 & -6.794 & -6.674 & 0.5684 & 0.8259 \\
\hline unknown & -8.269 & -8.053 & 0.5355 & 0.8347 & -6.828 & -6.923 & 0.8270 & 0.9282 \\
\hline unknown & -9.306 & -9.879 & 0.5925 & 0.8433 & -8.172 & -8.779 & 0.3721 & 0.7451 \\
\hline unknown & -8.296 & -8.766 & 0.5225 & 0.8300 & -6.945 & -6.910 & 0.9067 & 0.9580 \\
\hline unknown & -8.389 & -8.646 & 0.5577 & 0.8347 & -7.277 & -7.639 & 0.1475 & 0.5863 \\
\hline
\end{tabular}




\begin{tabular}{|c|c|c|c|c|c|c|c|c|}
\hline unknown & -0.851 & -0.746 & 0.7130 & 0.8917 & -0.671 & -0.262 & 0.0378 & 0.4440 \\
\hline unknown & -4.332 & -4.077 & 0.4692 & 0.8053 & -5.876 & -6.001 & 0.7761 & 0.9094 \\
\hline unknown & -6.763 & -6.394 & 0.4682 & 0.8053 & -9.249 & -9.225 & 0.9721 & 0.9900 \\
\hline unknown & -3.448 & -3.419 & 0.9222 & 0.9705 & -3.307 & -2.746 & 0.1022 & 0.5450 \\
\hline unknown & -9.429 & -10.264 & 0.3073 & 0.6837 & -9.158 & -9.609 & 0.5495 & 0.8138 \\
\hline unknown & -8.100 & -7.272 & 0.0481 & 0.4977 & -8.108 & -7.597 & 0.0229 & 0.4440 \\
\hline unknown & -7.963 & -7.983 & 0.9613 & 0.9820 & -5.891 & -6.246 & 0.5208 & 0.7986 \\
\hline unknown & -7.927 & -8.031 & 0.7027 & 0.8843 & -7.376 & -7.480 & 0.5986 & 0.8363 \\
\hline unknown & -1.844 & -1.834 & 0.9686 & 0.9827 & -1.697 & -1.266 & 0.0379 & 0.4440 \\
\hline unknown & 12.202 & 12.316 & 0.2848 & 0.6648 & 12.556 & 12.376 & 0.1096 & 0.5479 \\
\hline unknown & 4.098 & 4.289 & 0.7843 & 0.9091 & 10.382 & 7.892 & 0.1107 & 0.5479 \\
\hline unknown & -7.616 & -8.036 & 0.7829 & 0.9091 & -6.806 & -6.962 & 0.8408 & 0.9282 \\
\hline unknown & -7.669 & -8.803 & 0.4242 & 0.7757 & -5.749 & -6.628 & 0.2935 & 0.7040 \\
\hline unknown & -8.770 & -9.362 & 0.2747 & 0.6587 & -7.601 & -7.211 & 0.6759 & 0.8613 \\
\hline unknown & -7.798 & -6.864 & 0.0046 & 0.4977 & -7.494 & -7.045 & 0.2201 & 0.6419 \\
\hline unknown & -0.270 & -0.295 & 0.9288 & 0.9736 & -0.176 & 0.106 & 0.0184 & 0.4440 \\
\hline unknown & 11.305 & 11.565 & 0.3568 & 0.7286 & 11.577 & 11.604 & 0.7653 & 0.9046 \\
\hline unknown & -6.538 & -6.515 & 0.9382 & 0.9756 & -4.148 & -4.293 & 0.8205 & 0.9269 \\
\hline unknown & -5.567 & -4.817 & 0.1126 & 0.5435 & -5.346 & -4.816 & 0.1613 & 0.5933 \\
\hline unknown & -5.568 & -6.184 & 0.7510 & 0.9005 & -3.809 & -4.238 & 0.6564 & 0.8519 \\
\hline unknown & -7.895 & -8.922 & 0.4418 & 0.7899 & -6.543 & -7.322 & 0.2498 & 0.6776 \\
\hline unknown & 7.747 & 7.902 & 0.5426 & 0.8347 & 7.280 & 7.229 & 0.5655 & 0.8256 \\
\hline unknown & 8.815 & 9.671 & 0.0309 & 0.4977 & 8.639 & 8.746 & 0.3533 & 0.7373 \\
\hline unknown & 7.172 & 7.513 & 0.1750 & 0.5904 & 7.817 & 7.724 & 0.5413 & 0.8057 \\
\hline unknown & -7.548 & -6.917 & 0.0340 & 0.4977 & -5.711 & -5.630 & 0.8418 & 0.9282 \\
\hline unknown & -6.210 & -5.701 & 0.2271 & 0.6171 & -5.424 & -5.219 & 0.4372 & 0.7766 \\
\hline unknown & -5.008 & -4.707 & 0.4505 & 0.7949 & -4.728 & -4.318 & 0.1877 & 0.6198 \\
\hline unknown & -6.061 & -5.976 & 0.6162 & 0.8511 & -6.408 & -6.304 & 0.4906 & 0.7901 \\
\hline unknown & -7.626 & -7.073 & 0.2777 & 0.6587 & -7.226 & -6.689 & 0.2913 & 0.7025 \\
\hline unknown & -7.905 & -8.468 & 0.0876 & 0.5174 & -7.815 & -7.899 & 0.6247 & 0.8363 \\
\hline unknown & -7.226 & -6.856 & 0.3355 & 0.7135 & -6.528 & -6.673 & 0.6124 & 0.8363 \\
\hline unknown & -4.031 & -2.909 & 0.2150 & 0.6142 & -4.282 & -3.469 & 0.2171 & 0.6419 \\
\hline unknown & 6.912 & 7.409 & 0.0819 & 0.5174 & 7.613 & 7.572 & 0.8758 & 0.9406 \\
\hline unknown & -4.495 & -4.163 & 0.4060 & 0.7658 & -3.428 & -2.843 & 0.0653 & 0.4834 \\
\hline unknown & -8.814 & -8.875 & 0.9558 & 0.9819 & -8.127 & -8.081 & 0.9558 & 0.9803 \\
\hline unknown & -5.093 & -4.019 & 0.0743 & 0.5174 & -5.615 & -4.999 & 0.1150 & 0.5479 \\
\hline unknown & -6.689 & -5.618 & 0.0988 & 0.5435 & -6.187 & -5.380 & 0.2600 & 0.6791 \\
\hline unknown & -7.065 & -7.653 & 0.7291 & 0.8936 & -6.464 & -6.194 & 0.8143 & 0.9244 \\
\hline unknown & -7.883 & -8.086 & 0.8593 & 0.9394 & -7.456 & -7.808 & 0.6349 & 0.8408 \\
\hline unknown & -5.878 & -5.759 & 0.7871 & 0.9093 & -5.348 & -5.367 & 0.9356 & 0.9689 \\
\hline unknown & -5.977 & -5.818 & 0.7091 & 0.8880 & -5.457 & -5.565 & 0.3341 & 0.7310 \\
\hline unknown & -7.990 & -6.876 & 0.0355 & 0.4977 & -9.200 & -8.676 & 0.6396 & 0.8413 \\
\hline unknown & -7.386 & -6.123 & 0.0767 & 0.5174 & -7.911 & -7.497 & 0.6526 & 0.8497 \\
\hline unknown & -6.950 & -5.878 & 0.1789 & 0.5904 & -6.804 & -5.900 & 0.2152 & 0.6419 \\
\hline unknown & -8.231 & -8.211 & 0.9720 & 0.9833 & -7.827 & -7.562 & 0.3844 & 0.7540 \\
\hline unknown & -7.061 & -7.430 & 0.6767 & 0.8730 & -6.625 & -7.060 & 0.3736 & 0.7461 \\
\hline unknown & -5.871 & -6.093 & 0.7699 & 0.9050 & -5.632 & -6.068 & 0.3154 & 0.7175 \\
\hline unknown & -7.217 & -7.597 & 0.7545 & 0.9015 & -6.671 & -7.231 & 0.3171 & 0.7186 \\
\hline unknown & -5.170 & -4.630 & 0.0789 & 0.5174 & -4.507 & -3.900 & 0.0199 & 0.4440 \\
\hline unknown & -8.279 & -8.583 & 0.7313 & 0.8939 & -8.103 & -8.578 & 0.3932 & 0.7608 \\
\hline unknown & 7.713 & 7.657 & 0.6399 & 0.8547 & 8.061 & 8.016 & 0.8689 & 0.9390 \\
\hline unknown & 9.758 & 9.888 & 0.5751 & 0.8392 & 9.654 & 9.612 & 0.6310 & 0.8380 \\
\hline unknown & 6.445 & 7.292 & 0.2554 & 0.6427 & 8.046 & 7.782 & 0.7359 & 0.8944 \\
\hline unknown & -9.266 & -9.817 & 0.6389 & 0.8542 & -8.368 & -9.044 & 0.4541 & 0.7829 \\
\hline unknown & -6.855 & -7.505 & 0.1994 & 0.6008 & -6.855 & -6.063 & 0.3040 & 0.7132 \\
\hline unknown & -6.038 & -6.639 & 0.0389 & 0.4977 & -6.227 & -6.145 & 0.7830 & 0.9094 \\
\hline unknown & -8.093 & -8.616 & 0.6037 & 0.8495 & -6.989 & -7.855 & 0.3329 & 0.7310 \\
\hline
\end{tabular}




\begin{tabular}{|c|c|c|c|c|c|c|c|c|}
\hline unknown & -7.337 & -7.002 & 0.6058 & 0.8495 & -7.732 & -7.346 & 0.1318 & 0.5629 \\
\hline unknown & -6.606 & -6.161 & 0.1394 & 0.5584 & -6.555 & -6.326 & 0.1591 & 0.5926 \\
\hline unknown & -8.583 & -10.338 & 0.1740 & 0.5904 & -8.471 & -8.710 & 0.7926 & 0.9160 \\
\hline unknown & -8.342 & -8.868 & 0.1202 & 0.5435 & -8.529 & -8.350 & 0.6356 & 0.8408 \\
\hline unknown & -8.206 & -9.533 & 0.3181 & 0.6958 & -8.032 & -8.844 & 0.5210 & 0.7986 \\
\hline unknown & -7.837 & -8.167 & 0.2605 & 0.6427 & -7.238 & -7.183 & 0.7823 & 0.9094 \\
\hline unknown & 12.507 & 12.663 & 0.4504 & 0.7949 & 12.820 & 12.842 & 0.8371 & 0.9282 \\
\hline unknown & -8.226 & -8.723 & 0.5708 & 0.8363 & -7.913 & -8.430 & 0.5121 & 0.7935 \\
\hline unknown & -8.214 & -8.520 & 0.5666 & 0.8356 & -7.892 & -8.517 & 0.1305 & 0.5621 \\
\hline unknown & -4.991 & -4.950 & 0.7961 & 0.9122 & -5.054 & -4.919 & 0.3134 & 0.7163 \\
\hline unknown & -7.002 & -6.071 & 0.0091 & 0.4977 & -6.168 & -5.649 & 0.0390 & 0.4440 \\
\hline unknown & -7.431 & -9.148 & 0.3228 & 0.6973 & -8.050 & -7.145 & 0.4788 & 0.7846 \\
\hline unknown & -7.928 & -7.065 & 0.0982 & 0.5435 & -8.323 & -8.012 & 0.1987 & 0.6305 \\
\hline unknown & -11.015 & -12.878 & 0.2981 & 0.6729 & -4.123 & -3.381 & 0.1537 & 0.5918 \\
\hline unknown & -7.349 & -7.200 & 0.4625 & 0.7999 & -6.893 & -6.930 & 0.8748 & 0.9406 \\
\hline unknown & 7.328 & 7.554 & 0.4254 & 0.7757 & 8.421 & 8.378 & 0.8480 & 0.9294 \\
\hline unknown & -12.489 & -14.430 & 0.1617 & 0.5769 & -6.114 & -5.289 & 0.2454 & 0.6776 \\
\hline unknown & -7.576 & -7.973 & 0.6053 & 0.8495 & -7.796 & -8.526 & 0.2196 & 0.6419 \\
\hline unknown & -6.717 & -5.674 & 0.2333 & 0.6207 & -6.734 & -5.821 & 0.2884 & 0.7006 \\
\hline unknown & -7.048 & -6.137 & 0.0278 & 0.4977 & -6.979 & -6.452 & 0.1351 & 0.5638 \\
\hline unknown & 7.041 & 7.031 & 0.8537 & 0.9366 & 6.601 & 6.838 & 0.3496 & 0.7373 \\
\hline unknown & 6.940 & 6.978 & 0.6984 & 0.8830 & 6.197 & 6.753 & 0.1169 & 0.5479 \\
\hline unknown & 6.991 & 6.972 & 0.7508 & 0.9005 & 6.704 & 6.869 & 0.3862 & 0.7547 \\
\hline unknown & 5.500 & 5.562 & 0.5979 & 0.8472 & 6.505 & 6.704 & 0.6702 & 0.8591 \\
\hline unknown & 6.398 & 6.369 & 0.7054 & 0.8859 & 7.046 & 6.839 & 0.0937 & 0.5296 \\
\hline unknown & -5.044 & -5.130 & 0.6215 & 0.8511 & -5.430 & -5.242 & 0.3885 & 0.7575 \\
\hline unknown & 6.963 & 7.342 & 0.0677 & 0.5054 & 7.300 & 7.254 & 0.5440 & 0.8066 \\
\hline unknown & -5.066 & -4.479 & 0.3198 & 0.6958 & -5.539 & -4.709 & 0.1163 & 0.5479 \\
\hline unknown & -6.093 & -5.588 & 0.3130 & 0.6923 & -6.047 & -5.269 & 0.0317 & 0.4440 \\
\hline unknown & -3.186 & -2.604 & 0.0901 & 0.5227 & -2.647 & -2.234 & 0.2593 & 0.6791 \\
\hline unknown & -6.054 & -5.028 & 0.2778 & 0.6587 & -6.012 & -5.266 & 0.3285 & 0.7305 \\
\hline unknown & -9.493 & -9.763 & 0.7430 & 0.8999 & -9.796 & -9.626 & 0.8054 & 0.9201 \\
\hline unknown & -2.936 & -2.247 & 0.2206 & 0.6171 & -3.442 & -2.662 & 0.0926 & 0.5296 \\
\hline unknown & -3.191 & -2.449 & 0.2565 & 0.6427 & -3.765 & -2.813 & 0.0001 & 0.1476 \\
\hline unknown & -1.436 & -0.232 & 0.1371 & 0.5584 & -1.452 & -0.532 & 0.1021 & 0.5450 \\
\hline unknown & -9.167 & -7.693 & 0.1764 & 0.5904 & -10.589 & -9.733 & 0.1621 & 0.5942 \\
\hline unknown & -3.958 & -2.846 & 0.1150 & 0.5435 & -4.103 & -3.325 & 0.0528 & 0.4558 \\
\hline unknown & -8.999 & -8.103 & 0.0532 & 0.4977 & -8.332 & -8.246 & 0.6866 & 0.8666 \\
\hline unknown & -7.584 & -7.384 & 0.6556 & 0.8635 & -6.697 & -6.188 & 0.2105 & 0.6408 \\
\hline unknown & -8.028 & -7.836 & 0.7701 & 0.9050 & -7.486 & -7.589 & 0.7340 & 0.8944 \\
\hline unknown & -7.099 & -7.116 & 0.9671 & 0.9827 & -6.705 & -5.793 & 0.0809 & 0.5026 \\
\hline unknown & -2.238 & -1.881 & 0.3448 & 0.7217 & -2.434 & -1.964 & 0.0327 & 0.4440 \\
\hline unknown & -5.823 & -4.824 & 0.1369 & 0.5584 & -6.389 & -5.257 & 0.1291 & 0.5621 \\
\hline unknown & -5.077 & -4.510 & 0.0570 & 0.4977 & -5.808 & -5.446 & 0.2171 & 0.6419 \\
\hline unknown & -7.620 & -7.274 & 0.1368 & 0.5584 & -6.447 & -6.718 & 0.4187 & 0.7763 \\
\hline unknown & -9.114 & -9.377 & 0.8506 & 0.9366 & -9.214 & -9.841 & 0.4334 & 0.7766 \\
\hline unknown & -6.462 & -6.098 & 0.6541 & 0.8623 & -5.828 & -5.703 & 0.8156 & 0.9244 \\
\hline unknown & -6.115 & -6.486 & 0.3567 & 0.7286 & -5.093 & -5.097 & 0.9774 & 0.9920 \\
\hline unknown & -1.980 & -1.650 & 0.2250 & 0.6171 & -3.176 & -3.015 & 0.6687 & 0.8590 \\
\hline unknown & -8.874 & -8.540 & 0.6918 & 0.8787 & -8.271 & -8.166 & 0.8801 & 0.9428 \\
\hline unknown & -5.181 & -4.650 & 0.1104 & 0.5435 & -5.830 & -5.502 & 0.4761 & 0.7840 \\
\hline unknown & 7.361 & 7.757 & 0.1016 & 0.5435 & 8.059 & 8.057 & 0.9919 & 0.9990 \\
\hline unknown & -7.737 & -7.624 & 0.7685 & 0.9050 & -7.023 & -7.374 & 0.3463 & 0.7373 \\
\hline unknown & -7.751 & -7.352 & 0.4173 & 0.7691 & -7.585 & -6.474 & 0.0453 & 0.4440 \\
\hline unknown & -7.751 & -7.352 & 0.4173 & 0.7691 & -7.585 & -6.474 & 0.0453 & 0.4440 \\
\hline unknown & 10.715 & 10.667 & 0.8147 & 0.9172 & 10.844 & 10.714 & 0.1148 & 0.5479 \\
\hline unknown & 1.892 & 2.464 & 0.6221 & 0.8511 & 9.221 & 5.188 & 0.0655 & 0.4834 \\
\hline
\end{tabular}




\begin{tabular}{|c|c|c|c|c|c|c|c|c|}
\hline unknown & 8.688 & 8.568 & 0.7079 & 0.8880 & 8.898 & 8.730 & 0.0722 & 0.4936 \\
\hline unknown & -6.656 & -6.820 & 0.8717 & 0.9453 & -6.080 & -5.922 & 0.7935 & 0.9160 \\
\hline unknown & -5.589 & -5.795 & 0.9243 & 0.9705 & -4.548 & -4.240 & 0.7709 & 0.9073 \\
\hline unknown & -6.736 & -6.831 & 0.9369 & 0.9756 & -5.924 & -6.710 & 0.3726 & 0.7451 \\
\hline unknown & -5.211 & -5.199 & 0.9932 & 0.9965 & -4.305 & -4.295 & 0.9887 & 0.9970 \\
\hline unknown & -7.224 & -7.173 & 0.9671 & 0.9827 & -6.418 & -6.957 & 0.4430 & 0.7788 \\
\hline unknown & -3.386 & -3.607 & 0.4684 & 0.8053 & -3.407 & -3.421 & 0.9545 & 0.9803 \\
\hline unknown & -7.217 & -8.038 & 0.6900 & 0.8787 & -6.718 & -6.266 & 0.7646 & 0.9046 \\
\hline unknown & -7.367 & -7.278 & 0.9559 & 0.9819 & -6.378 & -6.504 & 0.8939 & 0.9524 \\
\hline unknown & -9.190 & -9.303 & 0.9243 & 0.9705 & -8.373 & -8.929 & 0.4672 & 0.7838 \\
\hline unknown & -5.124 & -5.076 & 0.8210 & 0.9172 & -5.272 & -4.943 & 0.0841 & 0.5105 \\
\hline unknown & 12.399 & 12.526 & 0.5327 & 0.8341 & 12.679 & 12.536 & 0.0973 & 0.5347 \\
\hline unknown & 5.080 & 5.234 & 0.8145 & 0.9172 & 8.344 & 6.455 & 0.0591 & 0.4631 \\
\hline unknown & 9.554 & 9.630 & 0.5865 & 0.8433 & 9.599 & 9.659 & 0.5654 & 0.8256 \\
\hline unknown & -9.636 & -9.867 & 0.8086 & 0.9148 & -8.802 & -9.255 & 0.5736 & 0.8282 \\
\hline unknown & -6.314 & -5.918 & 0.5645 & 0.8347 & -6.418 & -6.280 & 0.7348 & 0.8944 \\
\hline unknown & -8.666 & -9.570 & 0.6276 & 0.8514 & -7.694 & -8.089 & 0.5894 & 0.8363 \\
\hline unknown & -9.118 & -10.626 & 0.2580 & 0.6427 & -7.837 & -8.579 & 0.2645 & 0.6881 \\
\hline unknown & -2.226 & -2.462 & 0.1234 & 0.5486 & -2.045 & -2.096 & 0.7420 & 0.8963 \\
\hline unknown & -6.422 & -5.961 & 0.5999 & 0.8478 & -6.413 & -6.669 & 0.6068 & 0.8363 \\
\hline unknown & 6.602 & 6.641 & 0.8710 & 0.9453 & 6.962 & 6.844 & 0.0409 & 0.4440 \\
\hline unknown & -5.082 & -5.213 & 0.5445 & 0.8347 & -4.901 & -4.964 & 0.6412 & 0.8422 \\
\hline unknown & -4.422 & -3.371 & 0.0851 & 0.5174 & -5.520 & -5.848 & 0.4749 & 0.7840 \\
\hline unknown & -4.994 & -4.417 & 0.1895 & 0.5932 & -4.567 & -4.145 & 0.3486 & 0.7373 \\
\hline unknown & -7.366 & -7.311 & 0.8682 & 0.9445 & -6.887 & -7.287 & 0.2200 & 0.6419 \\
\hline unknown & 7.287 & 7.461 & 0.2089 & 0.6070 & 7.877 & 7.811 & 0.6243 & 0.8363 \\
\hline unknown & -7.609 & -5.516 & 0.1180 & 0.5435 & -8.597 & -8.021 & 0.1188 & 0.5479 \\
\hline unknown & -7.231 & -6.799 & 0.5403 & 0.8347 & -6.769 & -6.381 & 0.4827 & 0.7880 \\
\hline unknown & -8.671 & -9.436 & 0.5219 & 0.8300 & -7.425 & -8.302 & 0.4082 & 0.7728 \\
\hline unknown & -7.475 & -8.772 & 0.5095 & 0.8252 & -5.868 & -6.764 & 0.3592 & 0.7407 \\
\hline unknown & -7.538 & -8.941 & 0.5128 & 0.8274 & -5.813 & -6.756 & 0.3016 & 0.7120 \\
\hline unknown & -6.723 & -6.034 & 0.1138 & 0.5435 & -6.066 & -5.404 & 0.1204 & 0.5479 \\
\hline unknown & 9.826 & 10.561 & 0.0882 & 0.5174 & 9.006 & 8.914 & 0.8236 & 0.9278 \\
\hline unknown & -8.468 & -9.215 & 0.5536 & 0.8347 & -7.653 & -8.130 & 0.6235 & 0.8363 \\
\hline unknown & -1.865 & -1.632 & 0.4231 & 0.7757 & -0.691 & -0.566 & 0.7438 & 0.8975 \\
\hline unknown & -8.647 & -9.020 & 0.7509 & 0.9005 & -8.042 & -8.480 & 0.6419 & 0.8422 \\
\hline unknown & -3.641 & -3.579 & 0.8176 & 0.9172 & -3.653 & -3.388 & 0.3011 & 0.7120 \\
\hline unknown & -8.499 & -9.126 & 0.5889 & 0.8433 & -8.345 & -8.544 & 0.8241 & 0.9278 \\
\hline unknown & -7.929 & -6.351 & 0.0278 & 0.4977 & -8.683 & -8.090 & 0.2379 & 0.6686 \\
\hline unknown & -6.376 & -6.153 & 0.4235 & 0.7757 & -7.449 & -7.319 & 0.6638 & 0.8558 \\
\hline unknown & -5.303 & -4.910 & 0.3087 & 0.6852 & -6.847 & -6.602 & 0.5372 & 0.8037 \\
\hline unknown & 7.388 & 7.298 & 0.5531 & 0.8347 & 7.260 & 6.911 & 0.0060 & 0.4440 \\
\hline unknown & -7.429 & -7.802 & 0.0559 & 0.4977 & -7.597 & -7.469 & 0.3333 & 0.7310 \\
\hline unknown & -8.157 & -8.134 & 0.9558 & 0.9819 & -7.758 & -7.265 & 0.0295 & 0.4440 \\
\hline unknown & -8.817 & -8.075 & 0.4841 & 0.8099 & -8.157 & -8.166 & 0.9817 & 0.9937 \\
\hline unknown & -8.919 & -8.498 & 0.4255 & 0.7757 & -9.156 & -9.087 & 0.8616 & 0.9364 \\
\hline unknown & -8.883 & -7.888 & 0.1582 & 0.5769 & -8.880 & -8.710 & 0.7624 & 0.9029 \\
\hline unknown & 5.787 & 6.502 & 0.0425 & 0.4977 & 6.822 & 6.588 & 0.4190 & 0.7763 \\
\hline unknown & -8.669 & -9.944 & 0.4333 & 0.7800 & -8.323 & -8.141 & 0.7667 & 0.9055 \\
\hline unknown & -5.278 & -5.365 & 0.7987 & 0.9122 & -5.597 & -5.285 & 0.2554 & 0.6789 \\
\hline unknown & -7.854 & -8.344 & 0.6271 & 0.8514 & -7.168 & -7.804 & 0.4300 & 0.7766 \\
\hline unknown & -8.295 & -8.838 & 0.5527 & 0.8347 & -7.936 & -8.567 & 0.4188 & 0.7763 \\
\hline unknown & -7.110 & -6.978 & 0.6607 & 0.8642 & -6.338 & -6.143 & 0.4510 & 0.7820 \\
\hline unknown & -8.248 & -7.558 & 0.0572 & 0.4977 & -8.690 & -8.619 & 0.8375 & 0.9282 \\
\hline unknown & -5.998 & -6.215 & 0.2122 & 0.6124 & -5.998 & -5.909 & 0.6224 & 0.8363 \\
\hline unknown & 8.782 & 9.213 & 0.1396 & 0.5584 & 9.209 & 9.286 & 0.7130 & 0.8823 \\
\hline unknown & -6.813 & -5.907 & 0.0406 & 0.4977 & -7.089 & -6.397 & 0.0868 & 0.5106 \\
\hline
\end{tabular}




\begin{tabular}{|c|c|c|c|c|c|c|c|c|}
\hline unknown & -6.187 & -5.687 & 0.1854 & 0.5904 & -6.005 & -5.685 & 0.4204 & 0.7763 \\
\hline unknown & 8.239 & 8.527 & 0.2043 & 0.6030 & 8.134 & 8.200 & 0.8281 & 0.9282 \\
\hline unknown & 8.313 & 8.692 & 0.1600 & 0.5769 & 8.385 & 8.340 & 0.6901 & 0.8693 \\
\hline unknown & -8.201 & -7.921 & 0.6318 & 0.8542 & -7.877 & -7.226 & 0.2522 & 0.6776 \\
\hline unknown & -7.068 & -7.557 & 0.7203 & 0.8936 & -6.206 & -7.074 & 0.3539 & 0.7373 \\
\hline unknown & -6.510 & -7.328 & 0.5335 & 0.8341 & -5.984 & -6.705 & 0.4452 & 0.7798 \\
\hline unknown & -4.587 & -4.375 & 0.5052 & 0.8234 & -4.522 & -4.312 & 0.2823 & 0.6923 \\
\hline unknown & -6.907 & -6.434 & 0.2505 & 0.6379 & -6.248 & -5.328 & 0.0641 & 0.4834 \\
\hline unknown & -5.667 & -5.667 & 0.9999 & 0.9999 & -5.559 & -5.226 & 0.0364 & 0.4440 \\
\hline unknown & 9.222 & 8.630 & 0.0517 & 0.4977 & 9.476 & 9.052 & 0.0411 & 0.4440 \\
\hline unknown & 8.859 & 8.917 & 0.8187 & 0.9172 & 9.545 & 9.527 & 0.9502 & 0.9789 \\
\hline unknown & -9.119 & -9.945 & 0.5869 & 0.8433 & -8.461 & -9.313 & 0.3966 & 0.7621 \\
\hline unknown & -9.425 & -9.934 & 0.7279 & 0.8936 & -8.771 & -9.872 & 0.4356 & 0.7766 \\
\hline unknown & -6.945 & -6.690 & 0.5264 & 0.8319 & -6.915 & -6.695 & 0.3358 & 0.7310 \\
\hline unknown & 9.465 & 9.668 & 0.3137 & 0.6928 & 9.291 & 9.184 & 0.5654 & 0.8256 \\
\hline unknown & -7.800 & -8.604 & 0.5467 & 0.8347 & -7.569 & -8.793 & 0.2662 & 0.6882 \\
\hline unknown & -3.649 & -3.200 & 0.1230 & 0.5486 & -3.281 & -2.665 & 0.1198 & 0.5479 \\
\hline unknown & -4.707 & -4.515 & 0.5740 & 0.8392 & -4.268 & -3.876 & 0.0324 & 0.4440 \\
\hline unknown & 7.360 & 7.343 & 0.9548 & 0.9819 & 7.723 & 7.884 & 0.4357 & 0.7766 \\
\hline unknown & -5.651 & -5.035 & 0.1122 & 0.5435 & -5.459 & -5.138 & 0.2042 & 0.6332 \\
\hline unknown & -7.150 & -6.925 & 0.6168 & 0.8511 & -6.481 & -6.011 & 0.0894 & 0.5194 \\
\hline unknown & -10.901 & -11.128 & 0.7635 & 0.9044 & -6.677 & -5.732 & 0.1708 & 0.5944 \\
\hline unknown & -1.544 & -1.485 & 0.7304 & 0.8936 & -1.031 & -0.867 & 0.4704 & 0.7838 \\
\hline unknown & -9.059 & -8.090 & 0.0439 & 0.4977 & -8.328 & -7.439 & 0.0434 & 0.4440 \\
\hline unknown & 7.856 & 7.588 & 0.4619 & 0.7999 & 7.931 & 7.997 & 0.7393 & 0.8948 \\
\hline unknown & -4.698 & -4.427 & 0.1453 & 0.5602 & -3.985 & -3.839 & 0.5775 & 0.8319 \\
\hline unknown & -6.738 & -6.907 & 0.4078 & 0.7660 & -6.539 & -6.404 & 0.2799 & 0.6923 \\
\hline unknown & 7.488 & 7.681 & 0.3478 & 0.7251 & 7.526 & 7.612 & 0.5386 & 0.8048 \\
\hline unknown & -7.902 & -7.771 & 0.8441 & 0.9332 & -7.111 & -6.603 & 0.2197 & 0.6419 \\
\hline unknown & -5.586 & -4.833 & 0.1655 & 0.5796 & -5.678 & -5.039 & 0.1202 & 0.5479 \\
\hline unknown & -0.663 & -0.848 & 0.4236 & 0.7757 & -0.466 & -0.304 & 0.3829 & 0.7540 \\
\hline unknown & -9.797 & -8.917 & 0.0363 & 0.4977 & -9.517 & -9.441 & 0.8653 & 0.9379 \\
\hline unknown & -5.639 & -5.662 & 0.9508 & 0.9804 & -5.278 & -4.749 & 0.1232 & 0.5532 \\
\hline unknown & -7.876 & -6.858 & 0.1013 & 0.5435 & -7.439 & -5.647 & 0.0130 & 0.4440 \\
\hline unknown & -1.774 & -0.559 & 0.1715 & 0.5901 & -2.893 & -1.256 & 0.0431 & 0.4440 \\
\hline unknown & -5.153 & -5.127 & 0.7090 & 0.8880 & -5.060 & -4.815 & 0.1375 & 0.5638 \\
\hline unknown & -9.308 & -8.509 & 0.2056 & 0.6030 & -8.383 & -8.031 & 0.4254 & 0.7766 \\
\hline unknown & -6.237 & -6.171 & 0.7665 & 0.9044 & -6.327 & -5.981 & 0.1983 & 0.6305 \\
\hline unknown & -5.745 & -5.660 & 0.7248 & 0.8936 & -4.866 & -4.414 & 0.2159 & 0.6419 \\
\hline unknown & -7.983 & -7.954 & 0.9611 & 0.9820 & -8.171 & -8.011 & 0.5668 & 0.8256 \\
\hline unknown & 6.861 & 6.694 & 0.7339 & 0.8955 & 6.509 & 6.353 & 0.7511 & 0.9011 \\
\hline unknown & -8.816 & -8.918 & 0.7606 & 0.9038 & -7.356 & -7.268 & 0.8011 & 0.9176 \\
\hline unknown & -8.100 & -7.361 & 0.0346 & 0.4977 & -7.597 & -7.571 & 0.8462 & 0.9290 \\
\hline unknown & -8.954 & -9.378 & 0.7630 & 0.9044 & -8.462 & -8.979 & 0.6191 & 0.8363 \\
\hline unknown & -3.037 & -3.293 & 0.2968 & 0.6726 & -2.367 & -2.130 & 0.1243 & 0.5542 \\
\hline unknown & -7.141 & -6.685 & 0.6036 & 0.8495 & -6.983 & -6.745 & 0.6133 & 0.8363 \\
\hline unknown & 7.281 & 7.485 & 0.3503 & 0.7264 & 6.831 & 7.034 & 0.4400 & 0.7766 \\
\hline unknown & -5.233 & -5.245 & 0.9622 & 0.9820 & -5.264 & -5.152 & 0.6246 & 0.8363 \\
\hline unknown & -5.604 & -4.387 & 0.0001 & 0.1153 & -5.806 & -5.642 & 0.4745 & 0.7840 \\
\hline unknown & 3.402 & 2.383 & 0.2969 & 0.6726 & 6.953 & 3.308 & 0.0290 & 0.4440 \\
\hline unknown & -8.179 & -6.419 & 0.0002 & 0.1153 & -8.390 & -8.227 & 0.6035 & 0.8363 \\
\hline unknown & -8.281 & -8.937 & 0.6017 & 0.8490 & -7.492 & -8.056 & 0.5087 & 0.7935 \\
\hline unknown & -2.607 & -2.530 & 0.7650 & 0.9044 & -2.368 & -2.094 & 0.0258 & 0.4440 \\
\hline unknown & -8.731 & -9.242 & 0.7229 & 0.8936 & -8.564 & -8.981 & 0.5867 & 0.8363 \\
\hline unknown & -8.333 & -8.794 & 0.7816 & 0.9091 & -8.845 & -9.443 & 0.5937 & 0.8363 \\
\hline unknown & 8.857 & 8.776 & 0.8063 & 0.9142 & 9.445 & 9.525 & 0.8140 & 0.924 \\
\hline unknown & -5.120 & -5.137 & 0.9241 & 0.9705 & -5.198 & -4.860 & 0.0105 & 0.4440 \\
\hline
\end{tabular}




\begin{tabular}{|c|c|c|c|c|c|c|c|c|}
\hline unknown & -5.741 & -4.947 & 0.0103 & 0.4977 & -6.252 & -5.799 & 0.2029 & 0.6332 \\
\hline unknown & -5.279 & -4.484 & 0.1058 & 0.5435 & -6.094 & -5.632 & 0.2016 & 0.6332 \\
\hline unknown & 9.031 & 9.155 & 0.4896 & 0.8119 & 8.854 & 8.661 & 0.2854 & 0.6954 \\
\hline unknown & -7.278 & -7.688 & 0.7999 & 0.9122 & -6.317 & -6.718 & 0.7066 & 0.8771 \\
\hline unknown & -7.248 & -8.149 & 0.5835 & 0.8433 & -6.204 & -7.192 & 0.3546 & 0.7373 \\
\hline unknown & -6.761 & -6.565 & 0.4887 & 0.8115 & -6.780 & -6.638 & 0.6156 & 0.8363 \\
\hline unknown & -8.373 & -9.091 & 0.6590 & 0.8635 & -8.525 & -9.377 & 0.4978 & 0.7924 \\
\hline unknown & 7.463 & 7.481 & 0.9399 & 0.9756 & 7.584 & 7.686 & 0.5904 & 0.8363 \\
\hline unknown & -7.302 & -7.665 & 0.2571 & 0.6427 & -7.224 & -7.159 & 0.8458 & 0.9290 \\
\hline unknown & -6.717 & -6.017 & 0.0787 & 0.5174 & -7.064 & -6.983 & 0.7582 & 0.9020 \\
\hline unknown & -8.957 & -9.103 & 0.9188 & 0.9705 & -8.294 & -8.620 & 0.7370 & 0.8944 \\
\hline unknown & -8.914 & -9.545 & 0.7026 & 0.8843 & -7.823 & -8.417 & 0.6164 & 0.8363 \\
\hline unknown & -8.157 & -8.870 & 0.6805 & 0.8762 & -7.504 & -7.940 & 0.6597 & 0.8538 \\
\hline unknown & -7.102 & -8.075 & 0.5361 & 0.8347 & -6.643 & -7.402 & 0.4403 & 0.7766 \\
\hline unknown & -5.994 & -6.374 & 0.8363 & 0.9280 & -4.759 & -5.599 & 0.4481 & 0.7820 \\
\hline unknown & -4.520 & -4.194 & 0.2052 & 0.6030 & -4.365 & -4.140 & 0.2477 & 0.6776 \\
\hline unknown & -7.959 & -8.594 & 0.7324 & 0.8944 & -7.033 & -7.946 & 0.5186 & 0.7975 \\
\hline unknown & -7.398 & -7.225 & 0.4063 & 0.7658 & -7.049 & -6.815 & 0.4482 & 0.7820 \\
\hline unknown & -7.461 & -7.225 & 0.3343 & 0.7132 & -6.988 & -6.913 & 0.7618 & 0.9029 \\
\hline unknown & -6.989 & -6.526 & 0.2015 & 0.6009 & -6.727 & -6.475 & 0.4170 & 0.7763 \\
\hline unknown & -7.756 & -7.404 & 0.1404 & 0.5584 & -7.378 & -7.063 & 0.0967 & 0.5335 \\
\hline unknown & -8.217 & -9.245 & 0.4303 & 0.7785 & -7.901 & -8.794 & 0.4318 & 0.7766 \\
\hline unknown & 8.024 & 8.020 & 0.9627 & 0.9820 & 6.860 & 7.549 & 0.1970 & 0.6302 \\
\hline unknown & 8.264 & 8.251 & 0.8466 & 0.9348 & 7.228 & 7.797 & 0.2042 & 0.6332 \\
\hline unknown & 7.829 & 7.868 & 0.6902 & 0.8787 & 7.384 & 7.695 & 0.3013 & 0.7120 \\
\hline unknown & 10.366 & 10.481 & 0.5894 & 0.8433 & 10.849 & 10.840 & 0.9386 & 0.9701 \\
\hline unknown & 7.501 & 7.481 & 0.7188 & 0.8935 & 7.958 & 7.538 & 0.1536 & 0.5918 \\
\hline unknown & 7.644 & 7.624 & 0.9228 & 0.9705 & 7.863 & 7.967 & 0.3211 & 0.7218 \\
\hline unknown & -7.687 & -7.407 & 0.6220 & 0.8511 & -7.976 & -7.356 & 0.0351 & 0.4440 \\
\hline unknown & -8.353 & -9.262 & 0.4808 & 0.8099 & -8.143 & -9.039 & 0.3348 & 0.7310 \\
\hline unknown & -7.697 & -8.346 & 0.6730 & 0.8720 & -7.152 & -8.057 & 0.3480 & 0.7373 \\
\hline unknown & -7.802 & -8.514 & 0.5746 & 0.8392 & -7.252 & -8.281 & 0.1750 & 0.5998 \\
\hline unknown & -2.343 & -2.040 & 0.3588 & 0.7286 & -2.516 & -2.348 & 0.2208 & 0.6425 \\
\hline unknown & -5.164 & -5.159 & 0.9871 & 0.9916 & -5.251 & -5.393 & 0.5053 & 0.7935 \\
\hline unknown & -5.626 & -5.458 & 0.6357 & 0.8542 & -5.048 & -4.816 & 0.2623 & 0.6836 \\
\hline unknown & -1.549 & -0.558 & 0.0446 & 0.4977 & -2.144 & -0.948 & 0.0507 & 0.4558 \\
\hline unknown & 7.979 & 8.026 & 0.8601 & 0.9395 & 8.760 & 8.749 & 0.9367 & 0.9689 \\
\hline unknown & -8.145 & -8.036 & 0.8199 & 0.9172 & -7.319 & -6.982 & 0.2854 & 0.6954 \\
\hline unknown & -4.315 & -3.481 & 0.0875 & 0.5174 & -4.779 & -3.967 & 0.0958 & 0.5332 \\
\hline unknown & -8.875 & -9.519 & 0.6237 & 0.8511 & -7.604 & -8.127 & 0.6096 & 0.8363 \\
\hline unknown & -6.948 & -6.691 & 0.3998 & 0.7599 & -7.373 & -6.773 & 0.0036 & 0.4440 \\
\hline unknown & -5.958 & -5.474 & 0.1605 & 0.5769 & -7.047 & -6.823 & 0.4838 & 0.7880 \\
\hline unknown & -2.433 & -2.725 & 0.5185 & 0.8297 & -1.951 & -1.508 & 0.0706 & 0.4936 \\
\hline unknown & 7.632 & 7.748 & 0.2763 & 0.6587 & 7.916 & 7.739 & 0.1537 & 0.5918 \\
\hline unknown & -4.854 & -4.971 & 0.7017 & 0.8843 & -4.734 & -4.712 & 0.8925 & 0.9522 \\
\hline unknown & -6.491 & -6.503 & 0.9613 & 0.9820 & -6.886 & -6.658 & 0.4403 & 0.7766 \\
\hline unknown & -8.620 & -7.926 & 0.1140 & 0.5435 & -9.371 & -8.879 & 0.2064 & 0.6341 \\
\hline unknown & 6.952 & 6.869 & 0.8542 & 0.9366 & 6.872 & 5.931 & 0.3024 & 0.7125 \\
\hline unknown & 7.581 & 8.073 & 0.1088 & 0.5435 & 8.260 & 8.129 & 0.6071 & 0.8363 \\
\hline unknown & -5.035 & -4.858 & 0.6717 & 0.8717 & -6.203 & -5.728 & 0.0236 & 0.4440 \\
\hline unknown & -3.218 & -2.690 & 0.3338 & 0.7132 & -3.307 & -2.515 & 0.0277 & 0.4440 \\
\hline unknown & -8.779 & -8.152 & 0.2282 & 0.6171 & -7.485 & -7.038 & 0.4202 & 0.7763 \\
\hline unknown & 7.094 & 7.285 & 0.3945 & 0.7555 & 6.499 & 6.560 & 0.8355 & 0.9282 \\
\hline unknown & -5.559 & -5.083 & 0.2917 & 0.6709 & -5.292 & -5.101 & 0.4812 & 0.7875 \\
\hline unknown & 7.079 & 7.906 & 0.0443 & 0.4977 & 6.605 & 6.860 & 0.3501 & 0.7373 \\
\hline unknown & -3.491 & -2.930 & 0.1572 & 0.5769 & -3.962 & -3.648 & 0.3547 & 0.7373 \\
\hline unknown & -2.554 & -2.257 & 0.3438 & 0.7217 & -2.606 & -2.078 & 0.1753 & 0.5998 \\
\hline
\end{tabular}




\begin{tabular}{|c|c|c|c|c|c|c|c|c|}
\hline unknown & 7.434 & 7.269 & 0.6630 & 0.8655 & 7.130 & 7.132 & 0.9975 & 0.9990 \\
\hline unknown & -5.058 & -5.189 & 0.5144 & 0.8274 & -5.175 & -4.931 & 0.3265 & 0.7278 \\
\hline unknown & -5.545 & -5.079 & 0.2789 & 0.6587 & -6.160 & -5.963 & 0.6294 & 0.8380 \\
\hline unknown & -9.703 & -9.806 & 0.8575 & 0.9382 & -7.382 & -7.210 & 0.7834 & 0.9094 \\
\hline unknown & -8.193 & -8.864 & 0.6276 & 0.8514 & -7.256 & -7.778 & 0.4730 & 0.7838 \\
\hline unknown & -1.879 & -1.500 & 0.1408 & 0.5584 & -1.790 & -1.416 & 0.0443 & 0.4440 \\
\hline unknown & -3.601 & -3.616 & 0.9638 & 0.9824 & -3.165 & -2.709 & 0.2122 & 0.6419 \\
\hline unknown & -5.019 & -4.717 & 0.1396 & 0.5584 & -4.837 & -4.482 & 0.0583 & 0.4631 \\
\hline unknown & -6.724 & -7.350 & 0.0339 & 0.4977 & -6.894 & -6.895 & 0.9934 & 0.9990 \\
\hline unknown & -7.844 & -7.617 & 0.5356 & 0.8347 & -7.316 & -7.116 & 0.4670 & 0.7838 \\
\hline unknown & -8.213 & -7.572 & 0.3625 & 0.7303 & -7.912 & -6.661 & 0.0204 & 0.4440 \\
\hline unknown & -7.346 & -8.841 & 0.3762 & 0.7408 & -6.691 & -7.682 & 0.3672 & 0.7425 \\
\hline unknown & -6.976 & -6.643 & 0.6260 & 0.8514 & -6.928 & -6.150 & 0.1675 & 0.5944 \\
\hline unknown & -0.575 & -0.496 & 0.5445 & 0.8347 & -0.277 & -0.019 & 0.0922 & 0.5296 \\
\hline unknown & -4.567 & -3.217 & 0.2004 & 0.6008 & -4.543 & -4.277 & 0.2547 & 0.6786 \\
\hline unknown & -3.481 & -3.408 & 0.6760 & 0.8730 & -3.066 & -2.935 & 0.3918 & 0.7599 \\
\hline unknown & 12.766 & 12.832 & 0.7183 & 0.8935 & 13.169 & 13.084 & 0.3343 & 0.7310 \\
\hline unknown & -7.034 & -8.381 & 0.4137 & 0.7674 & -6.460 & -7.431 & 0.3512 & 0.7373 \\
\hline unknown & -7.281 & -8.434 & 0.4532 & 0.7964 & -6.615 & -7.284 & 0.4004 & 0.7671 \\
\hline unknown & -5.444 & -4.894 & 0.3581 & 0.7286 & -5.392 & -4.596 & 0.0083 & 0.4440 \\
\hline unknown & -4.876 & -4.566 & 0.5615 & 0.8347 & -4.869 & -4.057 & 0.0087 & 0.4440 \\
\hline unknown & -7.804 & -8.729 & 0.5031 & 0.8229 & -7.008 & -7.606 & 0.4954 & 0.7921 \\
\hline unknown & 0.009 & 0.236 & 0.2703 & 0.6524 & 0.159 & 0.426 & 0.0572 & 0.4631 \\
\hline unknown & -2.856 & -2.344 & 0.0556 & 0.4977 & -2.529 & -2.289 & 0.1377 & 0.5638 \\
\hline unknown & -9.250 & -10.035 & 0.5079 & 0.8248 & -8.425 & -8.793 & 0.6738 & 0.8610 \\
\hline unknown & 8.856 & 9.095 & 0.3384 & 0.7151 & 9.698 & 9.628 & 0.6986 & 0.8740 \\
\hline unknown & -5.419 & -5.198 & 0.4192 & 0.7714 & -6.155 & -6.110 & 0.9249 & 0.9645 \\
\hline unknown & -3.780 & -3.494 & 0.4544 & 0.7965 & -4.126 & -3.746 & 0.1396 & 0.5669 \\
\hline unknown & -7.553 & -8.439 & 0.6680 & 0.8694 & -5.587 & -6.283 & 0.2947 & 0.7056 \\
\hline unknown & 7.754 & 7.985 & 0.3294 & 0.7071 & 8.599 & 8.554 & 0.8162 & 0.9244 \\
\hline unknown & -7.968 & -7.561 & 0.3841 & 0.7475 & -8.489 & -7.973 & 0.1253 & 0.5563 \\
\hline unknown & -8.860 & -8.309 & 0.2602 & 0.6427 & -8.973 & -8.102 & 0.2274 & 0.6546 \\
\hline unknown & 8.067 & 8.097 & 0.7530 & 0.9012 & 8.110 & 8.049 & 0.5641 & 0.8256 \\
\hline unknown & -5.166 & -5.138 & 0.9425 & 0.9756 & -5.316 & -5.494 & 0.5119 & 0.7935 \\
\hline unknown & -3.799 & -3.794 & 0.9826 & 0.9893 & -4.123 & -3.965 & 0.4828 & 0.7880 \\
\hline unknown & -8.987 & -9.726 & 0.6231 & 0.8511 & -7.840 & -8.524 & 0.3770 & 0.7518 \\
\hline unknown & -6.478 & -5.470 & 0.0688 & 0.5054 & -7.104 & -6.300 & 0.0611 & 0.4676 \\
\hline unknown & -6.325 & -6.228 & 0.8202 & 0.9172 & -6.546 & -6.493 & 0.8005 & 0.9176 \\
\hline unknown & -7.523 & -6.519 & 0.1424 & 0.5602 & -7.454 & -6.394 & 0.0387 & 0.4440 \\
\hline unknown & -9.757 & -9.257 & 0.7878 & 0.9093 & -8.841 & -9.715 & 0.5302 & 0.8034 \\
\hline unknown & -6.720 & -6.522 & 0.5435 & 0.8347 & -9.039 & -9.585 & 0.4486 & 0.7820 \\
\hline unknown & -6.032 & -5.428 & 0.2156 & 0.6142 & -6.312 & -5.639 & 0.0944 & 0.5296 \\
\hline unknown & -6.888 & -5.634 & 0.0349 & 0.4977 & -7.883 & -7.269 & 0.2042 & 0.6332 \\
\hline unknown & 7.075 & 7.741 & 0.0186 & 0.4977 & 6.458 & 6.313 & 0.4926 & 0.7901 \\
\hline unknown & -5.986 & -5.592 & 0.1862 & 0.5904 & -6.882 & -6.755 & 0.6689 & 0.8590 \\
\hline unknown & 8.098 & 7.884 & 0.5908 & 0.8433 & 7.467 & 7.556 & 0.8435 & 0.9290 \\
\hline unknown & -6.692 & -6.356 & 0.5571 & 0.8347 & -7.127 & -6.406 & 0.0236 & 0.4440 \\
\hline unknown & -8.690 & -8.859 & 0.9108 & 0.9654 & -8.061 & -8.427 & 0.6615 & 0.8550 \\
\hline unknown & -8.497 & -8.767 & 0.8711 & 0.9453 & -8.005 & -8.425 & 0.6007 & 0.8363 \\
\hline unknown & -5.511 & -5.298 & 0.6382 & 0.8542 & -4.916 & -4.380 & 0.1489 & 0.5884 \\
\hline unknown & 10.587 & 10.702 & 0.6248 & 0.8514 & 10.550 & 10.508 & 0.4883 & 0.7897 \\
\hline unknown & -8.225 & -8.595 & 0.7602 & 0.9038 & -7.236 & -7.928 & 0.4493 & 0.7820 \\
\hline unknown & -4.029 & -3.077 & 0.0975 & 0.5435 & -3.206 & -1.929 & 0.0236 & 0.4440 \\
\hline unknown & 13.301 & 13.463 & 0.3063 & 0.6837 & 13.721 & 13.730 & 0.8773 & 0.9406 \\
\hline unknown & -6.066 & -5.160 & 0.0955 & 0.5406 & -5.464 & -4.560 & 0.0860 & 0.5105 \\
\hline unknown & -6.814 & -7.602 & 0.5990 & 0.8478 & -5.922 & -6.286 & 0.6805 & 0.8621 \\
\hline unknown & -8.252 & -10.534 & 0.3496 & 0.7260 & -6.696 & -8.672 & 0.1722 & 0.5944 \\
\hline
\end{tabular}




\begin{tabular}{|c|c|c|c|c|c|c|c|c|}
\hline unknown & -8.229 & -9.997 & 0.3380 & 0.7151 & -7.093 & -8.674 & 0.1347 & 0.5638 \\
\hline unknown & -4.721 & -3.751 & 0.0807 & 0.5174 & -5.338 & -4.956 & 0.1844 & 0.6159 \\
\hline unknown & -9.411 & -10.470 & 0.5208 & 0.8300 & -8.430 & -8.961 & 0.6258 & 0.8369 \\
\hline unknown & -1.136 & -0.768 & 0.2948 & 0.6726 & -0.527 & 0.199 & 0.0062 & 0.4440 \\
\hline unknown & 6.287 & 7.507 & 0.0597 & 0.4977 & 7.337 & 6.190 & 0.2663 & 0.6882 \\
\hline unknown & -7.422 & -8.170 & 0.6177 & 0.8511 & -6.807 & -7.348 & 0.6494 & 0.8471 \\
\hline unknown & -6.176 & -7.180 & 0.5859 & 0.8433 & -5.445 & -6.040 & 0.6399 & 0.8413 \\
\hline unknown & -6.300 & -6.678 & 0.8208 & 0.9172 & -5.421 & -5.722 & 0.7930 & 0.9160 \\
\hline unknown & -8.459 & -8.963 & 0.7140 & 0.8917 & -8.058 & -7.988 & 0.9359 & 0.9689 \\
\hline unknown & 6.510 & 7.567 & 0.0660 & 0.5051 & 7.291 & 6.318 & 0.2794 & 0.6923 \\
\hline unknown & -8.464 & -9.318 & 0.6226 & 0.8511 & -7.667 & -8.098 & 0.7532 & 0.9016 \\
\hline unknown & -0.324 & -0.357 & 0.9192 & 0.9705 & 0.026 & 0.650 & 0.0169 & 0.4440 \\
\hline unknown & 7.964 & 8.396 & 0.1380 & 0.5584 & 7.902 & 8.044 & 0.3357 & 0.7310 \\
\hline unknown & -5.422 & -4.888 & 0.2829 & 0.6635 & -5.247 & -4.531 & 0.0223 & 0.4440 \\
\hline unknown & -5.135 & -4.374 & 0.2330 & 0.6207 & -5.347 & -4.388 & 0.1179 & 0.5479 \\
\hline unknown & -6.615 & -6.396 & 0.5884 & 0.8433 & -7.617 & -7.115 & 0.0066 & 0.4440 \\
\hline unknown & -2.463 & -1.724 & 0.1793 & 0.5904 & -2.846 & -1.944 & 0.0123 & 0.4440 \\
\hline unknown & -2.109 & -1.412 & 0.1628 & 0.5769 & -2.460 & -1.867 & 0.1273 & 0.5579 \\
\hline unknown & 7.022 & 7.143 & 0.4088 & 0.7660 & 7.623 & 7.695 & 0.7112 & 0.8809 \\
\hline unknown & -7.511 & -8.013 & 0.1017 & 0.5435 & -7.439 & -7.232 & 0.0873 & 0.5112 \\
\hline unknown & -1.327 & -0.709 & 0.0615 & 0.4993 & -2.466 & -1.996 & 0.2357 & 0.6658 \\
\hline unknown & -1.498 & -1.115 & 0.2802 & 0.6594 & -1.691 & -1.204 & 0.0130 & 0.4440 \\
\hline unknown & -10.210 & -9.216 & 0.2456 & 0.6344 & -9.914 & -8.378 & 0.0487 & 0.4558 \\
\hline unknown & -7.422 & -7.993 & 0.0630 & 0.4993 & -7.485 & -7.604 & 0.3960 & 0.7621 \\
\hline unknown & -3.518 & -3.393 & 0.7513 & 0.9005 & -4.306 & -4.262 & 0.8752 & 0.9406 \\
\hline unknown & -1.042 & -0.650 & 0.1620 & 0.5769 & -1.841 & -1.643 & 0.4760 & 0.7840 \\
\hline unknown & 7.195 & 7.531 & 0.0874 & 0.5174 & 7.245 & 7.373 & 0.4981 & 0.7924 \\
\hline unknown & -4.128 & -3.752 & 0.2126 & 0.6124 & -5.052 & -4.906 & 0.4285 & 0.7766 \\
\hline unknown & -6.399 & -5.951 & 0.3732 & 0.7407 & -5.956 & -5.235 & 0.1128 & 0.5479 \\
\hline unknown & 11.783 & 11.634 & 0.5918 & 0.8433 & 12.056 & 11.830 & 0.0584 & 0.4631 \\
\hline unknown & -6.745 & -6.526 & 0.7905 & 0.9100 & -6.727 & -6.093 & 0.2857 & 0.6954 \\
\hline unknown & -6.982 & -7.147 & 0.6159 & 0.8511 & -6.811 & -6.422 & 0.3547 & 0.7373 \\
\hline unknown & -8.393 & -9.333 & 0.4873 & 0.8112 & -8.062 & -8.546 & 0.4504 & 0.7820 \\
\hline unknown & -4.415 & -4.086 & 0.3360 & 0.7135 & -6.582 & -6.628 & 0.9287 & 0.9666 \\
\hline unknown & 3.574 & 4.258 & 0.0841 & 0.5174 & 6.297 & 5.932 & 0.5121 & 0.7935 \\
\hline unknown & -7.069 & -6.698 & 0.3843 & 0.7475 & -10.409 & -10.214 & 0.7874 & 0.9116 \\
\hline unknown & -7.207 & -6.719 & 0.3115 & 0.6902 & -6.217 & -5.878 & 0.1040 & 0.5450 \\
\hline unknown & 13.448 & 13.562 & 0.5599 & 0.8347 & 13.722 & 13.661 & 0.1727 & 0.5944 \\
\hline unknown & -8.243 & -8.805 & 0.6425 & 0.8569 & -7.766 & -8.217 & 0.6240 & 0.8363 \\
\hline unknown & -5.781 & -4.957 & 0.2863 & 0.6661 & -6.285 & -5.260 & 0.0167 & 0.4440 \\
\hline unknown & -8.065 & -9.008 & 0.5270 & 0.8319 & -6.284 & -7.031 & 0.2715 & 0.6908 \\
\hline unknown & -7.303 & -7.764 & 0.7836 & 0.9091 & -6.178 & -6.607 & 0.5689 & 0.8259 \\
\hline unknown & 9.149 & 10.220 & 0.0105 & 0.4977 & 8.608 & 8.545 & 0.7458 & 0.8977 \\
\hline unknown & -6.001 & -5.275 & 0.1177 & 0.5435 & -6.837 & -6.187 & 0.2729 & 0.6908 \\
\hline unknown & 6.187 & 7.806 & 0.0132 & 0.4977 & 5.412 & 5.235 & 0.4859 & 0.7894 \\
\hline unknown & -5.082 & -4.478 & 0.1850 & 0.5904 & -4.595 & -3.926 & 0.0783 & 0.5026 \\
\hline unknown & 7.182 & 6.876 & 0.3581 & 0.7286 & 7.649 & 7.531 & 0.6357 & 0.8408 \\
\hline unknown & 8.534 & 8.724 & 0.2570 & 0.6427 & 9.138 & 9.103 & 0.8416 & 0.9282 \\
\hline unknown & -7.564 & -8.053 & 0.7296 & 0.8936 & -6.897 & -7.424 & 0.5751 & 0.8294 \\
\hline unknown & -5.607 & -4.568 & 0.1094 & 0.5435 & -6.338 & -5.370 & 0.0319 & 0.4440 \\
\hline unknown & -6.297 & -6.353 & 0.9780 & 0.9862 & -4.184 & -4.963 & 0.3436 & 0.7373 \\
\hline unknown & -6.219 & -7.451 & 0.6107 & 0.8511 & -4.335 & -5.188 & 0.3711 & 0.7451 \\
\hline unknown & 11.249 & 12.086 & 0.0592 & 0.4977 & 10.402 & 10.379 & 0.9595 & 0.9831 \\
\hline unknown & -8.462 & -7.256 & 0.1454 & 0.5602 & -9.423 & -8.349 & 0.0520 & 0.4558 \\
\hline unknown & -8.287 & -9.583 & 0.5270 & 0.8319 & -6.301 & -7.462 & 0.3407 & 0.7362 \\
\hline unknown & -4.454 & -3.579 & 0.2550 & 0.6427 & -5.173 & -4.497 & 0.0853 & 0.5105 \\
\hline unknown & 6.269 & 6.463 & 0.3594 & 0.7286 & 6.563 & 6.186 & 0.2892 & 0.7013 \\
\hline
\end{tabular}




\begin{tabular}{|c|c|c|c|c|c|c|c|c|}
\hline unknown & -7.246 & -5.620 & 0.0905 & 0.5227 & -7.951 & -7.153 & 0.1256 & 0.5563 \\
\hline unknown & -8.213 & -9.728 & 0.3145 & 0.6933 & -8.029 & -8.870 & 0.4153 & 0.7763 \\
\hline unknown & -7.833 & -8.718 & 0.5181 & 0.8297 & -7.344 & -8.200 & 0.4949 & 0.7921 \\
\hline unknown & -7.213 & -6.886 & 0.4408 & 0.7899 & -7.614 & -7.115 & 0.3048 & 0.7132 \\
\hline unknown & -9.022 & -9.086 & 0.9706 & 0.9833 & -8.139 & -8.015 & 0.8645 & 0.9379 \\
\hline unknown & -6.919 & -5.838 & 0.0878 & 0.5174 & -7.769 & -7.145 & 0.2051 & 0.6337 \\
\hline unknown & -6.491 & -6.761 & 0.2152 & 0.6142 & -7.063 & -6.727 & 0.4075 & 0.7728 \\
\hline unknown & -8.487 & -7.698 & 0.2279 & 0.6171 & -7.967 & -6.881 & 0.0400 & 0.4440 \\
\hline unknown & 7.181 & 7.202 & 0.6192 & 0.8511 & 7.154 & 7.064 & 0.3865 & 0.7547 \\
\hline unknown & -4.942 & -5.412 & 0.0414 & 0.4977 & -4.946 & -4.809 & 0.2695 & 0.6903 \\
\hline unknown & -2.873 & -1.908 & 0.1185 & 0.5435 & -2.387 & -1.512 & 0.0232 & 0.4440 \\
\hline unknown & -8.327 & -9.006 & 0.1242 & 0.5506 & -7.867 & -7.961 & 0.7709 & 0.9073 \\
\hline unknown & -1.816 & -1.210 & 0.2966 & 0.6726 & -2.395 & -0.845 & 0.0731 & 0.4936 \\
\hline unknown & 0.205 & 0.654 & 0.2370 & 0.6243 & 0.821 & 1.323 & 0.2672 & 0.6882 \\
\hline unknown & 9.492 & 9.859 & 0.1330 & 0.5584 & 9.634 & 9.658 & 0.8498 & 0.9294 \\
\hline unknown & -2.473 & -1.791 & 0.2044 & 0.6030 & -1.548 & -1.057 & 0.4612 & 0.7838 \\
\hline unknown & 3.447 & 3.850 & 0.5144 & 0.8274 & 6.684 & 6.238 & 0.4885 & 0.7897 \\
\hline unknown & 0.138 & 0.832 & 0.1029 & 0.5435 & 0.585 & 1.082 & 0.0846 & 0.5105 \\
\hline unknown & -2.076 & -1.240 & 0.3994 & 0.7599 & -0.857 & -0.614 & 0.0415 & 0.4440 \\
\hline unknown & 4.408 & 4.545 & 0.7848 & 0.9091 & 7.032 & 7.186 & 0.8348 & 0.9282 \\
\hline unknown & 10.802 & 10.979 & 0.3208 & 0.6965 & 10.696 & 10.573 & 0.4591 & 0.7838 \\
\hline unknown & -2.721 & -1.746 & 0.1098 & 0.5435 & -2.199 & -1.551 & 0.1368 & 0.5638 \\
\hline unknown & -4.134 & -4.208 & 0.8870 & 0.9539 & -3.565 & -3.391 & 0.3093 & 0.7135 \\
\hline unknown & 8.329 & 8.610 & 0.3353 & 0.7135 & 8.206 & 8.067 & 0.6990 & 0.8740 \\
\hline unknown & -7.800 & -8.219 & 0.7649 & 0.9044 & -7.356 & -7.841 & 0.5981 & 0.8363 \\
\hline unknown & -7.893 & -7.809 & 0.7832 & 0.9091 & -8.209 & -8.402 & 0.5046 & 0.7935 \\
\hline unknown & 6.263 & 7.703 & 0.0252 & 0.4977 & 4.576 & 5.598 & 0.3720 & 0.7451 \\
\hline unknown & -2.067 & -1.207 & 0.2160 & 0.6142 & -2.453 & -1.747 & 0.3537 & 0.7373 \\
\hline unknown & -7.408 & -6.529 & 0.0509 & 0.4977 & -7.298 & -7.028 & 0.1822 & 0.6159 \\
\hline unknown & 7.038 & 6.962 & 0.8373 & 0.9283 & 7.581 & 7.426 & 0.4852 & 0.7891 \\
\hline unknown & -4.617 & -4.168 & 0.4063 & 0.7658 & -5.287 & -4.683 & 0.3165 & 0.7186 \\
\hline unknown & -8.916 & -9.533 & 0.6591 & 0.8635 & -7.875 & -8.392 & 0.6181 & 0.8363 \\
\hline unknown & 6.489 & 6.589 & 0.4268 & 0.7757 & 7.250 & 6.844 & 0.1663 & 0.5944 \\
\hline unknown & -9.335 & -9.569 & 0.8521 & 0.9366 & -8.598 & -8.595 & 0.9972 & 0.9990 \\
\hline unknown & -8.173 & -7.331 & 0.1335 & 0.5584 & -8.015 & -7.416 & 0.1267 & 0.5575 \\
\hline unknown & -5.953 & -4.946 & 0.1348 & 0.5584 & -6.425 & -5.944 & 0.1942 & 0.6302 \\
\hline unknown & -8.514 & -9.308 & 0.5631 & 0.8347 & -7.922 & -8.261 & 0.7731 & 0.9081 \\
\hline unknown & -7.664 & -8.057 & 0.7953 & 0.9122 & -6.871 & -6.840 & 0.9785 & 0.9920 \\
\hline unknown & -8.085 & -8.347 & 0.8624 & 0.9397 & -7.241 & -6.907 & 0.7595 & 0.9020 \\
\hline unknown & 6.884 & 7.275 & 0.1194 & 0.5435 & 7.181 & 7.382 & 0.2813 & 0.6923 \\
\hline unknown & -3.536 & -2.803 & 0.2766 & 0.6587 & -4.351 & -3.467 & 0.0931 & 0.5296 \\
\hline unknown & -4.648 & -3.763 & 0.1206 & 0.5435 & -5.100 & -4.080 & 0.0454 & 0.4440 \\
\hline unknown & -6.671 & -6.123 & 0.4498 & 0.7949 & -6.995 & -6.301 & 0.1960 & 0.6302 \\
\hline unknown & -9.328 & -9.270 & 0.9621 & 0.9820 & -8.497 & -8.077 & 0.6769 & 0.8613 \\
\hline unknown & -8.015 & -8.966 & 0.5619 & 0.8347 & -6.835 & -7.181 & 0.6826 & 0.8640 \\
\hline unknown & -8.736 & -9.772 & 0.4589 & 0.7999 & -8.485 & -8.380 & 0.9131 & 0.9609 \\
\hline unknown & -8.924 & -9.837 & 0.4665 & 0.8049 & -8.472 & -8.778 & 0.7334 & 0.8944 \\
\hline unknown & -6.821 & -6.644 & 0.5707 & 0.8363 & -6.804 & -6.607 & 0.2807 & 0.6923 \\
\hline unknown & 7.447 & 6.756 & 0.0343 & 0.4977 & 7.531 & 7.202 & 0.1474 & 0.5863 \\
\hline unknown & -1.222 & -0.505 & 0.1617 & 0.5769 & -1.842 & -0.974 & 0.0338 & 0.4440 \\
\hline unknown & -7.639 & -7.577 & 0.8770 & 0.9486 & -7.797 & -7.262 & 0.1400 & 0.5669 \\
\hline unknown & -7.041 & -6.784 & 0.2685 & 0.6516 & -7.004 & -6.462 & 0.0831 & 0.5105 \\
\hline unknown & -3.942 & -2.772 & 0.0464 & 0.4977 & -5.316 & -4.197 & 0.0061 & 0.4440 \\
\hline unknown & -6.306 & -5.938 & 0.3626 & 0.7303 & -8.521 & -7.442 & 0.0960 & 0.5332 \\
\hline unknown & -6.425 & -5.562 & 0.1854 & 0.5904 & -8.691 & -7.060 & 0.0076 & 0.4440 \\
\hline unknown & -8.272 & -8.480 & 0.9099 & 0.9653 & -7.644 & -8.280 & 0.5310 & 0.8034 \\
\hline unknown & -7.946 & -8.202 & 0.9017 & 0.9596 & -6.874 & -7.400 & 0.6369 & 0.8408 \\
\hline
\end{tabular}




\begin{tabular}{|c|c|c|c|c|c|c|c|c|}
\hline unknown & 6.305 & 7.517 & 0.0041 & 0.4977 & 6.269 & 7.275 & 0.0408 & 0.4440 \\
\hline unknown & 6.583 & 6.668 & 0.2631 & 0.6445 & 7.496 & 7.485 & 0.9677 & 0.9879 \\
\hline unknown & -8.093 & -7.868 & 0.3218 & 0.6968 & -8.108 & -7.828 & 0.4071 & 0.7728 \\
\hline unknown & 8.971 & 8.880 & 0.5807 & 0.8433 & 8.455 & 8.259 & 0.2922 & 0.7034 \\
\hline unknown & -6.960 & -7.618 & 0.7862 & 0.9093 & -6.820 & -7.602 & 0.5130 & 0.7935 \\
\hline unknown & -7.433 & -8.150 & 0.7254 & 0.8936 & -6.953 & -7.672 & 0.4977 & 0.7924 \\
\hline unknown & -9.393 & -9.937 & 0.7513 & 0.9005 & -8.659 & -9.898 & 0.3625 & 0.7407 \\
\hline unknown & -6.782 & -8.368 & 0.3530 & 0.7286 & -6.538 & -7.284 & 0.5321 & 0.8034 \\
\hline unknown & -9.338 & -9.580 & 0.8776 & 0.9486 & -10.035 & -10.822 & 0.3905 & 0.7592 \\
\hline unknown & -8.651 & -9.673 & 0.3519 & 0.7286 & -8.583 & -9.203 & 0.2748 & 0.6923 \\
\hline unknown & -6.577 & -5.905 & 0.2367 & 0.6243 & -6.880 & -6.328 & 0.1164 & 0.5479 \\
\hline unknown & -8.597 & -8.598 & 0.9973 & 0.9988 & -7.681 & -7.521 & 0.5093 & 0.7935 \\
\hline unknown & 10.539 & 10.712 & 0.3298 & 0.7071 & 10.317 & 10.159 & 0.4118 & 0.7754 \\
\hline unknown & -6.937 & -7.536 & 0.7669 & 0.9044 & -6.595 & -7.647 & 0.4308 & 0.7766 \\
\hline unknown & -6.880 & -7.516 & 0.7559 & 0.9024 & -6.560 & -7.483 & 0.4591 & 0.7838 \\
\hline unknown & -5.311 & -5.715 & 0.0520 & 0.4977 & -5.381 & -5.250 & 0.2476 & 0.6776 \\
\hline unknown & -9.168 & -9.906 & 0.3569 & 0.7286 & -9.199 & -9.549 & 0.5565 & 0.8205 \\
\hline unknown & -7.203 & -6.330 & 0.1626 & 0.5769 & -8.375 & -7.754 & 0.1789 & 0.6090 \\
\hline unknown & -8.051 & -8.885 & 0.6237 & 0.8511 & -7.717 & -8.601 & 0.3615 & 0.7407 \\
\hline unknown & -8.631 & -8.667 & 0.9201 & 0.9705 & -7.486 & -7.378 & 0.6772 & 0.8613 \\
\hline unknown & 6.927 & 7.160 & 0.1791 & 0.5904 & 7.671 & 7.182 & 0.0820 & 0.5073 \\
\hline unknown & -5.891 & -6.221 & 0.1849 & 0.5904 & -5.536 & -5.188 & 0.0799 & 0.5026 \\
\hline unknown & -8.957 & -9.605 & 0.5940 & 0.8445 & -8.186 & -8.985 & 0.3618 & 0.7407 \\
\hline unknown & 5.270 & 5.705 & 0.1615 & 0.5769 & 6.148 & 5.569 & 0.3189 & 0.7192 \\
\hline unknown & -5.425 & -5.742 & 0.3375 & 0.7151 & -5.370 & -5.056 & 0.1666 & 0.5944 \\
\hline unknown & -8.202 & -7.182 & 0.1500 & 0.5646 & -8.605 & -7.350 & 0.0655 & 0.4834 \\
\hline unknown & -8.343 & -7.886 & 0.3074 & 0.6837 & -6.930 & -6.598 & 0.2531 & 0.6776 \\
\hline unknown & -2.146 & -0.558 & 0.1074 & 0.5435 & -2.293 & -1.033 & 0.0724 & 0.4936 \\
\hline unknown & -7.322 & -7.724 & 0.1129 & 0.5435 & -7.818 & -7.437 & 0.1970 & 0.6302 \\
\hline unknown & -4.729 & -3.665 & 0.0648 & 0.5021 & -5.025 & -3.943 & 0.0334 & 0.4440 \\
\hline unknown & -4.701 & -3.589 & 0.0487 & 0.4977 & -5.186 & -4.858 & 0.5271 & 0.8034 \\
\hline unknown & 8.625 & 8.753 & 0.0959 & 0.5406 & 8.759 & 8.795 & 0.7239 & 0.8891 \\
\hline unknown & 9.189 & 9.337 & 0.3427 & 0.7207 & 9.595 & 9.609 & 0.8890 & 0.9500 \\
\hline unknown & -5.268 & -5.243 & 0.9205 & 0.9705 & -5.526 & -5.245 & 0.2183 & 0.6419 \\
\hline unknown & -6.475 & -6.520 & 0.8346 & 0.9272 & -6.565 & -6.225 & 0.0446 & 0.4440 \\
\hline unknown & -9.988 & -10.190 & 0.8649 & 0.9417 & -8.884 & -9.150 & 0.8048 & 0.9201 \\
\hline unknown & -9.026 & -8.838 & 0.5591 & 0.8347 & -8.615 & -8.471 & 0.6057 & 0.8363 \\
\hline unknown & -8.598 & -8.966 & 0.7959 & 0.9122 & -7.699 & -7.757 & 0.9508 & 0.9789 \\
\hline unknown & -5.626 & -5.431 & 0.6050 & 0.8495 & -6.434 & -5.528 & 0.0318 & 0.4440 \\
\hline unknown & 6.642 & 6.713 & 0.7774 & 0.9091 & 7.348 & 7.345 & 0.9851 & 0.9963 \\
\hline unknown & 6.181 & 6.419 & 0.2912 & 0.6709 & 6.249 & 5.925 & 0.1905 & 0.6261 \\
\hline unknown & -7.464 & -8.082 & 0.6831 & 0.8771 & -6.466 & -6.734 & 0.7718 & 0.9076 \\
\hline unknown & -9.236 & -9.735 & 0.6536 & 0.8623 & -8.279 & -9.012 & 0.4435 & 0.7788 \\
\hline unknown & -7.285 & -7.587 & 0.8718 & 0.9453 & -6.086 & -6.261 & 0.8455 & 0.9290 \\
\hline unknown & -6.600 & -7.177 & 0.2489 & 0.6362 & -6.520 & -6.234 & 0.2763 & 0.6923 \\
\hline unknown & 6.817 & 6.917 & 0.1310 & 0.5584 & 6.998 & 6.924 & 0.5354 & 0.8035 \\
\hline unknown & -7.573 & -7.952 & 0.8038 & 0.9126 & -6.962 & -7.051 & 0.9302 & 0.9675 \\
\hline unknown & -8.204 & -8.499 & 0.8798 & 0.9486 & -7.297 & -7.205 & 0.9259 & 0.9645 \\
\hline unknown & 3.718 & 3.281 & 0.6381 & 0.8542 & 7.155 & 7.076 & 0.9057 & 0.9577 \\
\hline unknown & -8.130 & -8.628 & 0.7439 & 0.9001 & -7.890 & -7.951 & 0.9523 & 0.9791 \\
\hline unknown & -8.913 & -8.968 & 0.9736 & 0.9833 & -8.503 & -8.066 & 0.6543 & 0.8510 \\
\hline unknown & -6.309 & -6.088 & 0.6337 & 0.8542 & -6.461 & -5.848 & 0.0534 & 0.4558 \\
\hline unknown & 5.307 & 5.865 & 0.1289 & 0.5584 & 6.473 & 5.764 & 0.2706 & 0.6908 \\
\hline unknown & 4.628 & 3.762 & 0.3802 & 0.7462 & 6.816 & 7.398 & 0.3086 & 0.7132 \\
\hline unknown & -5.819 & -5.938 & 0.6199 & 0.8511 & -6.020 & -5.625 & 0.0661 & 0.4834 \\
\hline unknown & -6.596 & -6.380 & 0.4042 & 0.7651 & -7.329 & -7.003 & 0.1833 & 0.6159 \\
\hline unknown & -5.527 & -5.549 & 0.9016 & 0.9596 & -5.666 & -5.374 & 0.0577 & 0.4631 \\
\hline
\end{tabular}




\begin{tabular}{|c|c|c|c|c|c|c|c|c|}
\hline unknown & 6.916 & 6.911 & 0.9811 & 0.9886 & 7.231 & 7.016 & 0.3239 & 0.7256 \\
\hline unknown & -7.612 & -7.865 & 0.9051 & 0.9617 & -7.211 & -7.479 & 0.7737 & 0.9081 \\
\hline unknown & -7.797 & -8.046 & 0.9011 & 0.9596 & -7.386 & -8.126 & 0.5885 & 0.8363 \\
\hline unknown & -6.846 & -6.380 & 0.3172 & 0.6958 & -7.794 & -7.474 & 0.2478 & 0.6776 \\
\hline unknown & -4.398 & -4.562 & 0.5047 & 0.8234 & -4.673 & -4.467 & 0.2392 & 0.6695 \\
\hline unknown & 9.051 & 9.316 & 0.0133 & 0.4977 & 9.225 & 9.356 & 0.1805 & 0.6128 \\
\hline unknown & 11.413 & 11.477 & 0.1113 & 0.5435 & 11.706 & 11.777 & 0.3626 & 0.7407 \\
\hline unknown & -7.751 & -7.798 & 0.9259 & 0.9714 & -8.076 & -7.511 & 0.0084 & 0.4440 \\
\hline unknown & -3.604 & -3.641 & 0.8445 & 0.9332 & -3.673 & -3.434 & 0.0745 & 0.4957 \\
\hline unknown & 7.843 & 8.051 & 0.3153 & 0.6940 & 8.524 & 8.486 & 0.8560 & 0.9336 \\
\hline unknown & -7.954 & -8.212 & 0.3851 & 0.7475 & -8.602 & -8.808 & 0.5879 & 0.8363 \\
\hline unknown & -7.757 & -7.859 & 0.7977 & 0.9122 & -8.707 & -8.305 & 0.0726 & 0.4936 \\
\hline unknown & -8.319 & -9.240 & 0.4687 & 0.8053 & -7.626 & -8.338 & 0.4147 & 0.7763 \\
\hline unknown & -8.275 & -8.710 & 0.1523 & 0.5715 & -8.290 & -8.117 & 0.6286 & 0.8380 \\
\hline unknown & -7.159 & -7.038 & 0.7672 & 0.9044 & -7.340 & -7.063 & 0.2906 & 0.7021 \\
\hline unknown & -8.460 & -9.082 & 0.6703 & 0.8708 & -8.050 & -8.839 & 0.4538 & 0.7829 \\
\hline unknown & -6.135 & -6.423 & 0.2920 & 0.6709 & -6.410 & -6.284 & 0.4238 & 0.7766 \\
\hline unknown & -8.898 & -9.569 & 0.6151 & 0.8511 & -8.671 & -9.399 & 0.4123 & 0.7754 \\
\hline unknown & 5.516 & 5.858 & 0.3757 & 0.7408 & 8.014 & 7.535 & 0.4203 & 0.7763 \\
\hline unknown & 6.131 & 6.231 & 0.1185 & 0.5435 & 6.762 & 6.182 & 0.0422 & 0.4440 \\
\hline unknown & -8.794 & -9.470 & 0.4738 & 0.8060 & -8.658 & -9.502 & 0.3653 & 0.7407 \\
\hline unknown & -8.176 & -9.332 & 0.3249 & 0.7000 & -7.814 & -8.848 & 0.2411 & 0.6719 \\
\hline unknown & 6.741 & 6.836 & 0.2466 & 0.6356 & 6.088 & 6.472 & 0.2096 & 0.6408 \\
\hline unknown & -8.605 & -9.136 & 0.6939 & 0.8790 & -8.281 & -9.591 & 0.2189 & 0.6419 \\
\hline unknown & -7.787 & -7.778 & 0.9733 & 0.9833 & -8.264 & -7.891 & 0.3113 & 0.7149 \\
\hline unknown & -8.540 & -9.358 & 0.5893 & 0.8433 & -8.426 & -9.326 & 0.3833 & 0.7540 \\
\hline unknown & 5.220 & 5.781 & 0.2000 & 0.6008 & 6.647 & 5.262 & 0.1172 & 0.5479 \\
\hline unknown & -7.221 & -7.423 & 0.5596 & 0.8347 & -7.561 & -7.062 & 0.0470 & 0.4545 \\
\hline unknown & -7.312 & -7.271 & 0.8322 & 0.9266 & -8.209 & -7.908 & 0.1653 & 0.5944 \\
\hline unknown & -9.138 & -8.817 & 0.4832 & 0.8099 & -8.532 & -7.934 & 0.1729 & 0.5944 \\
\hline unknown & 9.491 & 9.548 & 0.7847 & 0.9091 & 9.383 & 9.273 & 0.1159 & 0.5479 \\
\hline unknown & 7.723 & 7.700 & 0.8823 & 0.9497 & 7.979 & 7.858 & 0.1335 & 0.5638 \\
\hline unknown & -7.542 & -7.234 & 0.2321 & 0.6207 & -7.819 & -7.612 & 0.2930 & 0.7040 \\
\hline unknown & -7.125 & -6.661 & 0.1888 & 0.5932 & -6.880 & -6.116 & 0.0195 & 0.4440 \\
\hline unknown & 12.020 & 12.185 & 0.0755 & 0.5174 & 12.227 & 12.190 & 0.5709 & 0.8269 \\
\hline unknown & 9.300 & 9.361 & 0.4852 & 0.8107 & 9.261 & 9.213 & 0.5971 & 0.8363 \\
\hline unknown & 6.440 & 6.534 & 0.2662 & 0.6483 & 6.532 & 6.373 & 0.1678 & 0.5944 \\
\hline unknown & 9.267 & 9.460 & 0.0603 & 0.4984 & 9.066 & 9.258 & 0.3652 & 0.7407 \\
\hline unknown & -6.682 & -5.762 & 0.0869 & 0.5174 & -7.399 & -6.929 & 0.1688 & 0.5944 \\
\hline unknown & -5.617 & -5.604 & 0.9577 & 0.9820 & -5.123 & -4.722 & 0.0431 & 0.4440 \\
\hline unknown & -9.351 & -9.232 & 0.9328 & 0.9754 & -9.329 & -8.938 & 0.6591 & 0.8538 \\
\hline unknown & 7.808 & 8.035 & 0.1823 & 0.5904 & 8.427 & 8.355 & 0.6994 & 0.8740 \\
\hline unknown & 6.398 & 6.980 & 0.0501 & 0.4977 & 5.389 & 5.649 & 0.7514 & 0.9011 \\
\hline unknown & -5.112 & -5.542 & 0.2295 & 0.6180 & -5.084 & -4.679 & 0.1351 & 0.5638 \\
\hline unknown & 6.190 & 5.702 & 0.1010 & 0.5435 & 7.334 & 7.617 & 0.5715 & 0.8269 \\
\hline unknown & -7.036 & -6.712 & 0.4726 & 0.8059 & -7.376 & -6.913 & 0.1118 & 0.5479 \\
\hline unknown & 5.246 & 5.701 & 0.4974 & 0.8203 & 8.459 & 7.954 & 0.4331 & 0.7766 \\
\hline unknown & -9.058 & -9.385 & 0.7904 & 0.9100 & -7.908 & -8.586 & 0.4885 & 0.7897 \\
\hline unknown & 4.073 & 3.687 & 0.8268 & 0.9221 & 8.112 & 7.449 & 0.5355 & 0.8035 \\
\hline unknown & -6.142 & -5.889 & 0.5959 & 0.8453 & -7.003 & -6.289 & 0.0238 & 0.4440 \\
\hline unknown & -5.374 & -5.044 & 0.3680 & 0.7354 & -5.721 & -5.228 & 0.0755 & 0.4986 \\
\hline unknown & -9.412 & -10.425 & 0.2557 & 0.6427 & -8.797 & -9.181 & 0.6795 & 0.8618 \\
\hline unknown & -7.509 & -8.497 & 0.5707 & 0.8363 & -6.376 & -7.102 & 0.4649 & 0.783 \\
\hline unknown & 5.283 & 4.174 & 0.3977 & 0.7593 & 7.746 & 7.212 & 0.5334 & 0.803 \\
\hline unknown & -8.731 & -9.157 & 0.7185 & 0.8935 & -8.237 & -8.906 & 0.4682 & 0.783 \\
\hline unknown & -7.616 & -8.200 & 0.0835 & 0.5174 & -7.596 & -7.419 & 0.3948 & 0.760 \\
\hline unknown & -4.435 & -4.549 & 0.6928 & 0.8787 & -4.796 & -4.342 & 0.0414 & 0.4440 \\
\hline
\end{tabular}




\begin{tabular}{|c|c|c|c|c|c|c|c|c|}
\hline unknown & -4.639 & -4.610 & 0.8997 & 0.9596 & -5.651 & -5.410 & 0.2102 & 0.6408 \\
\hline unknown & -8.726 & -8.155 & 0.2417 & 0.6276 & -8.461 & -7.853 & 0.0205 & 0.4440 \\
\hline unknown & -8.744 & -9.162 & 0.7536 & 0.9012 & -8.258 & -8.740 & 0.6298 & 0.8380 \\
\hline unknown & -8.630 & -9.319 & 0.5817 & 0.8433 & -8.099 & -8.550 & 0.6426 & 0.8424 \\
\hline unknown & -9.536 & -9.331 & 0.8308 & 0.9258 & -8.659 & -8.510 & 0.8353 & 0.9282 \\
\hline unknown & -7.479 & -8.094 & 0.6751 & 0.8727 & -6.731 & -7.335 & 0.5789 & 0.8323 \\
\hline unknown & -4.516 & -4.584 & 0.7807 & 0.9091 & -5.600 & -5.388 & 0.5042 & 0.7935 \\
\hline unknown & -5.679 & -5.805 & 0.6733 & 0.8720 & -6.400 & -6.412 & 0.9600 & 0.9831 \\
\hline unknown & -8.082 & -7.557 & 0.1967 & 0.6005 & -8.237 & -7.383 & 0.0856 & 0.5105 \\
\hline unknown & -7.013 & -7.033 & 0.9403 & 0.9756 & -6.980 & -6.631 & 0.0679 & 0.4901 \\
\hline unknown & 10.490 & 10.402 & 0.5546 & 0.8347 & 10.615 & 10.571 & 0.2513 & 0.6776 \\
\hline unknown & 7.931 & 7.843 & 0.6969 & 0.8819 & 8.156 & 8.122 & 0.7976 & 0.9174 \\
\hline unknown & -6.957 & -6.880 & 0.7936 & 0.9122 & -9.959 & -9.491 & 0.5400 & 0.8057 \\
\hline unknown & -7.201 & -7.238 & 0.9208 & 0.9705 & -6.989 & -6.723 & 0.2721 & 0.6908 \\
\hline unknown & 12.151 & 12.288 & 0.1595 & 0.5769 & 12.312 & 12.208 & 0.2520 & 0.6776 \\
\hline unknown & -6.144 & -5.660 & 0.1951 & 0.5980 & -7.017 & -6.412 & 0.2585 & 0.6791 \\
\hline unknown & 7.491 & 8.460 & 0.0075 & 0.4977 & 6.407 & 6.464 & 0.8487 & 0.9294 \\
\hline unknown & 6.308 & 5.936 & 0.6459 & 0.8589 & 5.914 & 5.571 & 0.6953 & 0.8726 \\
\hline unknown & 7.611 & 7.761 & 0.4257 & 0.7757 & 8.031 & 8.002 & 0.8269 & 0.9282 \\
\hline unknown & -7.519 & -6.659 & 0.1431 & 0.5602 & -8.032 & -7.108 & 0.0379 & 0.4440 \\
\hline unknown & -8.674 & -9.573 & 0.5633 & 0.8347 & -7.264 & -7.939 & 0.3892 & 0.7579 \\
\hline unknown & -8.637 & -9.560 & 0.5537 & 0.8347 & -7.323 & -8.091 & 0.4091 & 0.7728 \\
\hline unknown & 9.405 & 10.033 & 0.0407 & 0.4977 & 8.490 & 8.460 & 0.9525 & 0.9791 \\
\hline unknown & -8.342 & -7.696 & 0.2420 & 0.6276 & -9.137 & -8.347 & 0.1067 & 0.5479 \\
\hline unknown & 7.288 & 7.183 & 0.1853 & 0.5904 & 7.390 & 7.248 & 0.2085 & 0.6390 \\
\hline unknown & 6.253 & 6.441 & 0.2067 & 0.6046 & 7.797 & 7.029 & 0.1558 & 0.5918 \\
\hline unknown & -7.099 & -6.982 & 0.7247 & 0.8936 & -7.511 & -6.903 & 0.0338 & 0.4440 \\
\hline unknown & -5.707 & -6.152 & 0.1087 & 0.5435 & -5.694 & -5.601 & 0.4645 & 0.7838 \\
\hline unknown & -6.293 & -5.542 & 0.1745 & 0.5904 & -6.045 & -5.188 & 0.0097 & 0.4440 \\
\hline unknown & -5.707 & -4.818 & 0.0573 & 0.4977 & -5.503 & -4.773 & 0.2714 & 0.6908 \\
\hline unknown & -4.239 & -4.295 & 0.8555 & 0.9368 & -4.147 & -3.761 & 0.0520 & 0.4558 \\
\hline unknown & 8.546 & 8.907 & 0.0879 & 0.5174 & 8.570 & 8.549 & 0.8384 & 0.9282 \\
\hline unknown & -7.183 & -7.423 & 0.5074 & 0.8248 & -7.220 & -6.610 & 0.0193 & 0.4440 \\
\hline unknown & -4.404 & -4.254 & 0.6236 & 0.8511 & -4.253 & -3.828 & 0.0518 & 0.4558 \\
\hline unknown & 7.035 & 7.237 & 0.3600 & 0.7286 & 6.607 & 6.397 & 0.3986 & 0.7649 \\
\hline unknown & 9.965 & 10.204 & 0.1308 & 0.5584 & 9.750 & 9.674 & 0.6785 & 0.8618 \\
\hline unknown & -7.541 & -7.533 & 0.9867 & 0.9916 & -7.365 & -6.937 & 0.1848 & 0.6159 \\
\hline unknown & 6.880 & 7.330 & 0.2216 & 0.6171 & 6.682 & 6.454 & 0.4605 & 0.7838 \\
\hline unknown & 8.011 & 8.188 & 0.3888 & 0.7510 & 7.882 & 8.039 & 0.6510 & 0.8484 \\
\hline unknown & -8.083 & -7.889 & 0.4607 & 0.7999 & -7.994 & -7.595 & 0.0551 & 0.4631 \\
\hline unknown & -6.749 & -7.059 & 0.4137 & 0.7674 & -7.696 & -7.543 & 0.7563 & 0.9020 \\
\hline unknown & 7.377 & 7.477 & 0.1891 & 0.5932 & 7.359 & 7.539 & 0.2159 & 0.6419 \\
\hline unknown & 3.900 & 4.164 & 0.2076 & 0.6047 & 6.995 & 6.269 & 0.3612 & 0.7407 \\
\hline unknown & -8.966 & -8.158 & 0.1497 & 0.5646 & -8.689 & -8.256 & 0.0998 & 0.5380 \\
\hline unknown & 8.197 & 8.143 & 0.7408 & 0.8999 & 7.619 & 7.535 & 0.4548 & 0.4548 \\
\hline unknown & 6.494 & 6.815 & 0.2349 & 0.6213 & 6.791 & 6.991 & 0.2596 & 0.6791 \\
\hline unknown & -6.477 & -6.286 & 0.7302 & 0.8936 & -7.491 & -6.681 & 0.0490 & 0.4558 \\
\hline unknown & -7.410 & -6.714 & 0.1991 & 0.6008 & -7.885 & -6.933 & 0.0368 & 0.4440 \\
\hline unknown & 6.192 & 6.594 & 0.1292 & 0.5584 & 8.333 & 6.990 & 0.1195 & 0.5479 \\
\hline unknown & 5.406 & 4.535 & 0.4825 & 0.8099 & 7.644 & 7.024 & 0.4750 & 0.7840 \\
\hline unknown & -7.303 & -7.240 & 0.8434 & 0.9332 & -7.221 & -7.012 & 0.1941 & 0.6302 \\
\hline unknown & 7.240 & 6.524 & 0.0359 & 0.4977 & 7.261 & 6.917 & 0.0347 & 0.4440 \\
\hline unknown & -4.514 & -4.368 & 0.6518 & 0.8623 & -4.990 & -4.302 & 0.0089 & 0.4440 \\
\hline unknown & -11.297 & -11.765 & 0.5451 & 0.8347 & -8.400 & -7.955 & 0.5789 & 0.8323 \\
\hline unknown & 5.149 & 4.092 & 0.4305 & 0.7785 & 6.223 & 5.655 & 0.3535 & 0.7373 \\
\hline unknown & -6.450 & -5.758 & 0.1377 & 0.5584 & -7.789 & -6.746 & 0.0048 & 0.4440 \\
\hline unknown & -9.232 & -9.770 & 0.6831 & 0.8771 & -8.153 & -8.701 & 0.5576 & 0.8212 \\
\hline
\end{tabular}




\begin{tabular}{lcccccccc} 
unknown & -7.750 & -8.370 & 0.0861 & 0.5174 & -8.081 & -8.019 & 0.8416 & 0.9282 \\
unknown & 5.309 & 5.083 & 0.8790 & 0.9486 & 6.960 & 5.992 & 0.2310 & 0.6619 \\
unknown & -7.824 & -8.385 & 0.7218 & 0.8936 & -6.703 & -6.990 & 0.7565 & 0.9020 \\
unknown & -9.002 & -9.911 & 0.4560 & 0.7971 & -8.585 & -8.477 & 0.9000 & 0.9541 \\
unknown & -7.815 & -7.726 & 0.5202 & 0.8300 & -7.701 & -7.370 & 0.1069 & 0.5479 \\
unknown & 8.130 & 7.958 & 0.3647 & 0.7303 & 7.414 & 7.290 & 0.5811 & 0.8335 \\
unknown & -9.339 & -9.834 & 0.7407 & 0.8999 & -9.531 & -10.030 & 0.5327 & 0.8034 \\
unknown & 9.810 & 9.971 & 0.2892 & 0.6709 & 9.334 & 9.283 & 0.7836 & 0.9094 \\
unknown & -7.250 & -8.081 & 0.0424 & 0.4977 & -7.572 & -7.382 & 0.3799 & 0.7530 \\
unknown & -8.703 & -9.588 & 0.5685 & 0.8363 & -8.985 & -9.813 & 0.4559 & 0.7829 \\
unknown & -9.343 & -9.565 & 0.8902 & 0.9550 & -8.965 & -10.065 & 0.3078 & 0.7132 \\
unknown & 6.383 & 5.899 & 0.3753 & 0.7408 & 5.621 & 5.578 & 0.8995 & 0.9541 \\
unknown & -6.975 & -7.651 & 0.0547 & 0.4977 & -6.782 & -6.500 & 0.3638 & 0.7407 \\
unknown & -6.942 & -6.684 & 0.4491 & 0.7949 & -6.833 & -6.442 & 0.0282 & 0.4440 \\
unknown & -6.574 & -7.038 & 0.2786 & 0.6587 & -6.592 & -6.253 & 0.2340 & 0.6658 \\
unknown & 5.523 & 5.067 & 0.4738 & 0.8060 & 6.468 & 5.781 & 0.3602 & 0.7407 \\
unknown & -6.435 & -5.642 & 0.1791 & 0.5904 & -6.988 & -5.939 & 0.0054 & 0.4440 \\
unknown & -8.445 & -7.735 & 0.1143 & 0.5435 & -8.833 & -7.828 & 0.0253 & 0.4440 \\
unknown & 7.455 & 7.634 & 0.0502 & 0.4977 & 7.574 & 7.676 & 0.2789 & 0.6923 \\
unknown & 7.726 & 7.802 & 0.5294 & 0.8321 & 8.037 & 8.120 & 0.1986 & 0.6305 \\
unknown & -6.896 & -7.094 & 0.4325 & 0.7798 & -7.166 & -6.820 & 0.1842 & 0.6159 \\
unknown & -7.784 & -7.930 & 0.5887 & 0.8433 & -7.859 & -7.506 & 0.1190 & 0.5479 \\
\hline
\end{tabular}

${ }^{\mathrm{a}}$ The ambiguous subspecies indicates that the lipidomic profiling gave two possible identifications of the lipid. ${ }^{\mathrm{b}}$ Abbreviations: Cer, ceramide. HexCer, hexosylceramide. SM, sphingomyelin. LysoPC, lysophophosphatidylcholine. LysoPE, lysophosphatidylethanolamine. PC, phosphatidylcholine. PE, phosphatidylethanolamine. PI, phosphatidylinositol. 
Online Resource Table S5

Results from the analysis of variance between the control group $(\mathrm{CON})$ and the high-energy feeding group (HIGH) within time points in adipose tissue positive electrospray ionization mode (ESI+) dataset. $P$-values were obtained from the analysis performed using MIXED procedure in SAS, with diet as the fixed effect and pair as the random effect. Adjusted- $p$ values were obtained from $p$-values after false discovery rate control.

\begin{tabular}{|c|c|c|c|c|c|c|c|c|c|c|c|c|}
\hline \multirow[b]{2}{*}{ Lipid subspecies } & \multicolumn{4}{|c|}{$-8 \mathrm{~d}$} & \multicolumn{4}{|c|}{$1 \mathrm{~d}$} & \multicolumn{4}{|c|}{$9 \mathrm{~d}$} \\
\hline & $\mathrm{CON}$ & HIGH & $p$-value & Adjusted- $p$ & $\mathrm{CON}$ & HIGH & $p$-value & Adjusted- $p$ & $\mathrm{CON}$ & HIGH & $p$-value & Adjusted- $p$ \\
\hline ChoE(14:0) & 4.565 & 4.544 & 0.8680 & 0.9010 & 4.666 & 4.594 & 0.3060 & 0.8154 & 4.408 & 4.459 & 0.5440 & 0.6318 \\
\hline ChoE(18:0) & 7.545 & 7.474 & 0.4980 & 0.6339 & 7.639 & 7.486 & 0.2990 & 0.8154 & 7.455 & 7.530 & 0.4980 & 0.5937 \\
\hline LysoPC(16:0) & -3.193 & -3.226 & 0.6910 & 0.7868 & -3.177 & -3.182 & 0.9660 & 0.9774 & -3.246 & -3.433 & 0.0700 & 0.5288 \\
\hline LysoPC(18:0) & -4.799 & -4.876 & 0.4460 & 0.5887 & -4.685 & -4.841 & 0.2880 & 0.8154 & -4.907 & -5.046 & 0.3800 & 0.5291 \\
\hline $\mathrm{PC}(32: 0)$ & -3.760 & -3.758 & 0.9750 & 0.9818 & -3.497 & -3.703 & 0.1980 & 0.8154 & -3.671 & -3.693 & 0.8840 & 0.9127 \\
\hline $\mathrm{PC}(32: 1)$ & -7.208 & -7.100 & 0.8230 & 0.8727 & -6.782 & -7.150 & 0.4120 & 0.8154 & -6.673 & -6.888 & 0.7570 & 0.8153 \\
\hline $\mathrm{PC}(34: 1)$ & -4.609 & -4.675 & 0.8860 & 0.9143 & -3.921 & -4.798 & 0.1530 & 0.8154 & -3.786 & -3.924 & 0.8720 & 0.9037 \\
\hline $\mathrm{PC}(34: 2)$ & -6.340 & -6.566 & 0.5670 & 0.6907 & -5.731 & -6.563 & 0.1500 & 0.8154 & -5.310 & -5.599 & 0.6720 & 0.7426 \\
\hline $\mathrm{PC}(36: 2)$ & -6.008 & -5.994 & 0.9710 & 0.9804 & -5.161 & -6.045 & 0.1790 & 0.8154 & -5.221 & -5.156 & 0.9290 & 0.9431 \\
\hline PC(38:3) & -7.066 & -6.881 & 0.7220 & 0.8053 & -6.464 & -6.805 & 0.6050 & 0.8391 & -7.073 & -6.837 & 0.6030 & 0.6850 \\
\hline PC(38:4) & -7.171 & -6.973 & 0.6430 & 0.7485 & -6.746 & -7.018 & 0.5900 & 0.8339 & -7.382 & -6.905 & 0.5070 & 0.5957 \\
\hline $\operatorname{SM}(\mathrm{d} 18: 1 / 16: 0)$ & -4.856 & -5.315 & 0.2790 & 0.5110 & -4.109 & -5.534 & 0.0210 & 0.8154 & -4.723 & -3.958 & 0.2770 & 0.5288 \\
\hline $\mathrm{SM}(\mathrm{d} 18: 1 / 24: 0)$ & -5.114 & -5.699 & 0.2780 & 0.5110 & -4.745 & -5.873 & 0.0090 & 0.8154 & -4.949 & -4.545 & 0.3420 & 0.5291 \\
\hline $\operatorname{SM}(\mathrm{d} 18: 1 / 24: 1)$ & -5.760 & -6.045 & 0.6060 & 0.7216 & -5.691 & -6.585 & 0.0240 & 0.8154 & -5.680 & -4.806 & 0.1250 & 0.5288 \\
\hline TG(14:0/16:0/17:0)+ & -3.409 & -3.613 & 0.4600 & 0.5931 & -3.297 & -3.193 & 0.7230 & 0.8935 & -3.698 & -3.094 & 0.1540 & 0.5288 \\
\hline TG(14:0/18:0/15:0) $)^{\mathrm{a}}$ & & & & & & & & & & & & \\
\hline $\mathrm{TG}(14: 0 / 16: 0 / 17: 0)+$ & -2.526 & -2.942 & 0.2870 & 0.5110 & -2.406 & -2.320 & 0.8000 & 0.9305 & -3.016 & -2.283 & 0.2310 & 0.5288 \\
\hline $\mathrm{TG}(14: 0 / 18: 0 / 15: 0)^{\mathrm{a}}$ & & & & & & & & & & & & \\
\hline TG(17:0/18:1/18:1) & -4.997 & -6.207 & 0.0730 & 0.5110 & -4.731 & -5.096 & 0.3110 & 0.8154 & -6.207 & -5.126 & 0.3960 & 0.5291 \\
\hline TG(17:1/18:1/18:2) & -2.804 & -3.763 & 0.1880 & 0.5110 & -2.434 & -3.167 & 0.1700 & 0.8154 & -4.059 & -2.901 & 0.3290 & 0.5288 \\
\hline TG(18:1/18:2/18:1) & 0.603 & -0.271 & 0.2070 & 0.5110 & 0.965 & 0.284 & 0.1480 & 0.8154 & -0.323 & 0.462 & 0.4040 & 0.5307 \\
\hline TG(44:1) & -1.656 & -2.333 & 0.3380 & 0.5293 & -1.576 & -1.408 & 0.6980 & 0.8844 & -2.644 & -1.407 & 0.2080 & 0.5288 \\
\hline TG(45:0) & -3.929 & -4.305 & 0.2410 & 0.5110 & -3.951 & -3.693 & 0.1960 & 0.8154 & -4.138 & -3.726 & 0.3740 & 0.5291 \\
\hline TG(45:1) & -3.811 & -4.494 & 0.2610 & 0.5110 & -3.842 & -3.491 & 0.3200 & 0.8154 & -4.217 & -3.726 & 0.5060 & 0.5957 \\
\hline TG(46:1) & 0.487 & -0.268 & 0.2720 & 0.5110 & 0.547 & 0.716 & 0.7010 & 0.8844 & -0.665 & 0.689 & 0.1850 & 0.5288 \\
\hline TG(46:3) & -4.563 & -5.638 & 0.2280 & 0.5110 & -4.090 & -4.763 & 0.4100 & 0.8154 & -5.690 & -4.637 & 0.3560 & 0.5291 \\
\hline TG(47:1) & -1.844 & -2.510 & 0.2900 & 0.5110 & -1.766 & -1.474 & 0.3620 & 0.8154 & -2.418 & -1.679 & 0.3650 & 0.5291 \\
\hline TG(47:2) & -2.339 & -3.092 & 0.2820 & 0.5110 & -2.097 & -2.304 & 0.6610 & 0.8614 & -3.137 & -2.279 & 0.3280 & 0.5288 \\
\hline $\mathrm{TG}(47: 3)$ & -6.133 & -6.986 & 0.2400 & 0.5110 & -5.840 & -6.503 & 0.3620 & 0.8154 & -6.854 & -6.343 & 0.5370 & 0.6244 \\
\hline $\mathrm{TG}(48: 1)$ & 1.959 & 1.226 & 0.2540 & 0.5110 & 2.143 & 2.177 & 0.9270 & 0.9664 & 0.846 & 2.139 & 0.1900 & 0.5288 \\
\hline $\mathrm{TG}(48: 2)$ & 1.892 & 1.151 & 0.2580 & 0.5110 & 2.168 & 1.999 & 0.6520 & 0.8614 & 0.676 & 2.012 & 0.2080 & 0.5288 \\
\hline TG(48:4) & -5.049 & -5.916 & 0.2630 & 0.5110 & -4.816 & -5.201 & 0.5280 & 0.8240 & -6.340 & -5.086 & 0.2790 & 0.5288 \\
\hline TG(49:0) & -3.306 & -3.833 & 0.3800 & 0.5441 & -3.163 & -3.068 & 0.7440 & 0.9067 & -3.773 & -3.244 & 0.4150 & 0.5374 \\
\hline TG(49:2) & -0.496 & -1.240 & 0.3050 & 0.5110 & -0.136 & -0.342 & 0.5790 & 0.8317 & -1.546 & -0.392 & 0.2990 & 0.5288 \\
\hline TG(50:4) & -2.075 & -2.981 & 0.2300 & 0.5110 & -1.724 & -2.204 & 0.4190 & 0.8154 & -3.317 & -2.130 & 0.3140 & 0.5288 \\
\hline TG(50:5) & -5.214 & -6.052 & 0.0960 & 0.5110 & -4.911 & -5.740 & 0.1990 & 0.8154 & -5.847 & -5.608 & 0.7090 & 0.7698 \\
\hline $\mathrm{TG}(51: 2)$ & 1.095 & 0.240 & 0.2030 & 0.5110 & 1.397 & 1.210 & 0.4610 & 0.8202 & -0.051 & 1.126 & 0.2980 & 0.5288 \\
\hline $\mathrm{TG}(51: 2)$ & -3.427 & -4.323 & 0.2130 & 0.5110 & -3.212 & -3.332 & 0.7480 & 0.9079 & -4.572 & -3.462 & 0.3450 & 0.5291 \\
\hline TG(51:4) & -4.308 & -5.200 & 0.1780 & 0.5110 & -4.009 & -4.348 & 0.4920 & 0.8215 & -5.399 & -4.301 & 0.3220 & 0.5288 \\
\hline $\mathrm{TG}(52: 2)$ & 4.042 & 3.499 & 0.2530 & 0.5110 & 4.300 & 4.083 & 0.3820 & 0.8154 & 3.102 & 4.098 & 0.2510 & 0.5288 \\
\hline $\mathrm{TG}(52: 3)$ & 2.410 & 1.700 & 0.2640 & 0.5110 & 2.861 & 2.405 & 0.2760 & 0.8154 & 1.301 & 2.379 & 0.3090 & 0.5288 \\
\hline $\mathrm{TG}(52: 4)$ & -0.317 & -1.091 & 0.2560 & 0.5110 & 0.052 & -0.314 & 0.4380 & 0.8154 & -1.360 & -0.362 & 0.3360 & 0.5288 \\
\hline $\operatorname{TG}(53: 2)$ & 0.476 & -0.400 & 0.1970 & 0.5110 & 0.844 & 0.467 & 0.2120 & 0.8154 & -0.586 & 0.534 & 0.3370 & 0.5288 \\
\hline $\operatorname{TG}(53: 2)$ & 0.147 & -0.692 & 0.1890 & 0.5110 & 0.643 & 0.189 & 0.2020 & 0.8154 & -0.840 & 0.098 & 0.3930 & 0.5291 \\
\hline TG(53:3) & -4.944 & -6.061 & 0.1440 & 0.5110 & -4.544 & -5.217 & 0.1320 & 0.8154 & -6.488 & -5.074 & 0.3400 & 0.5291 \\
\hline TG(53:5) & -4.741 & -5.385 & 0.0590 & 0.5110 & -4.438 & -5.005 & 0.2260 & 0.8154 & -5.128 & -5.064 & 0.9120 & 0.9340 \\
\hline TG(54:0) & -4.986 & -5.829 & 0.1710 & 0.5110 & -4.842 & -5.128 & 0.6180 & 0.8492 & -5.723 & -4.991 & 0.4670 & 0.5671 \\
\hline TG(54:5) & -1.425 & -2.424 & 0.1290 & 0.5110 & -1.116 & -1.691 & 0.2510 & 0.8154 & -2.086 & -1.769 & 0.6830 & 0.7470 \\
\hline TG(54:6) & -4.486 & -5.439 & 0.1250 & 0.5110 & -4.195 & -4.689 & 0.4010 & 0.8154 & -4.872 & -4.755 & 0.8620 & 0.8966 \\
\hline TG(55:4) & -4.759 & -5.682 & 0.1560 & 0.5110 & -4.377 & -4.856 & 0.2400 & 0.8154 & -5.887 & -4.928 & 0.3820 & 0.5291 \\
\hline TG(55:5) & -5.964 & -6.471 & 0.1610 & 0.5110 & -5.630 & -6.172 & 0.2050 & 0.8154 & -6.375 & -6.131 & 0.6740 & 0.7431 \\
\hline TG(56:4) & -3.615 & -4.831 & 0.1190 & 0.5110 & -3.489 & -3.921 & 0.3550 & 0.8154 & -4.571 & -4.084 & 0.5450 & 0.6318 \\
\hline TG(56:5) & -4.095 & -5.111 & 0.0530 & 0.5110 & -4.027 & -4.530 & 0.1580 & 0.8154 & -4.828 & -4.487 & 0.5780 & 0.6615 \\
\hline TG(56:5) & -5.316 & -5.752 & 0.1320 & 0.5110 & -5.000 & -5.350 & 0.3170 & 0.8154 & -5.995 & -5.149 & 0.2210 & 0.5288 \\
\hline TG(56:6) & -3.647 & -4.754 & 0.0920 & 0.5110 & -3.247 & -3.905 & 0.1970 & 0.8154 & -4.468 & -3.725 & 0.4180 & 0.5391 \\
\hline $\mathrm{TG}(58: 7)$ & -6.783 & -8.301 & 0.0630 & 0.5110 & -6.297 & -7.312 & 0.2120 & 0.8154 & -7.284 & -7.143 & 0.8830 & 0.9127 \\
\hline unknown & -5.049 & -5.200 & 0.2320 & 0.5110 & -4.942 & -5.385 & 0.2040 & 0.8154 & -5.488 & -5.154 & 0.0920 & 0.5288 \\
\hline unknown & -4.214 & -4.272 & 0.5600 & 0.6854 & -4.175 & -4.254 & 0.5780 & 0.8317 & -4.390 & -4.448 & 0.2500 & 0.5288 \\
\hline unknown & -6.672 & -7.149 & 0.1820 & 0.5110 & -6.372 & -6.751 & 0.1990 & 0.8154 & -7.203 & -6.810 & 0.4440 & 0.5572 \\
\hline unknown & -6.384 & -6.858 & 0.1940 & 0.5110 & -6.274 & -6.602 & 0.3740 & 0.8154 & -6.999 & -6.359 & 0.1920 & 0.5288 \\
\hline unknown & -6.217 & -6.661 & 0.2720 & 0.5110 & -5.942 & -6.307 & 0.0090 & 0.8154 & -6.789 & -6.083 & 0.2080 & 0.5288 \\
\hline unknown & -3.487 & -3.429 & 0.5460 & 0.6722 & -3.379 & -3.456 & 0.4190 & 0.8154 & -3.477 & -3.606 & 0.2610 & 0.5288 \\
\hline unknown & -4.087 & -4.248 & 0.1810 & 0.5110 & -3.965 & -4.122 & 0.3570 & 0.8154 & -4.297 & -4.334 & 0.6690 & 0.7414 \\
\hline unknown & -5.240 & -5.344 & 0.4530 & 0.5920 & -5.248 & -5.427 & 0.1160 & 0.8154 & -5.382 & -5.505 & 0.4190 & 0.5403 \\
\hline unknown & -3.916 & -4.025 & 0.4120 & 0.5667 & -3.909 & -3.960 & 0.6270 & 0.8537 & -3.890 & -4.163 & 0.0500 & 0.5288 \\
\hline unknown & -3.909 & -3.941 & 0.8100 & 0.8667 & -3.778 & -3.836 & 0.6700 & 0.8614 & -3.941 & -3.912 & 0.7990 & 0.8478 \\
\hline unknown & -5.671 & -5.794 & 0.2060 & 0.5110 & -5.637 & -5.626 & 0.9280 & 0.9664 & -5.649 & -5.774 & 0.4320 & 0.5495 \\
\hline unknown & -5.084 & -5.077 & 0.9500 & 0.9665 & -4.944 & -5.038 & 0.3220 & 0.8154 & -5.047 & -5.183 & 0.2450 & 0.5288 \\
\hline unknown & -2.520 & -2.557 & 0.7040 & 0.8002 & -2.467 & -2.591 & 0.2130 & 0.8154 & -2.587 & -2.646 & 0.4340 & 0.5510 \\
\hline unknown & -2.696 & -2.743 & 0.7770 & 0.8443 & -2.852 & -2.841 & 0.9520 & 0.9744 & -2.757 & -3.011 & 0.0660 & 0.5288 \\
\hline
\end{tabular}




\begin{tabular}{|c|c|c|c|c|c|c|c|c|c|c|c|c|}
\hline unknown & -4.829 & -4.818 & 0.9310 & 0.9542 & -4.712 & -4.766 & 0.7400 & 0.9039 & -4.659 & -5.042 & 0.0390 & 0.5288 \\
\hline unknown & -5.782 & -6.305 & 0.2310 & 0.5110 & -5.430 & -5.859 & 0.1660 & 0.8154 & -6.481 & -5.730 & 0.3310 & 0.5288 \\
\hline unknown & -6.244 & -6.773 & 0.2530 & 0.5110 & -5.825 & -6.227 & 0.2620 & 0.8154 & -6.903 & -6.206 & 0.3190 & 0.5288 \\
\hline unknown & -6.984 & -7.426 & 0.2820 & 0.5110 & -6.755 & -7.080 & 0.3780 & 0.8154 & -7.673 & -7.050 & 0.3950 & 0.5291 \\
\hline unknown & -1.079 & -1.113 & 0.7690 & 0.8382 & -0.967 & -1.033 & 0.4850 & 0.8215 & -1.090 & -1.212 & 0.2980 & 0.5288 \\
\hline unknown & -2.972 & -3.015 & 0.7350 & 0.8094 & -2.821 & -2.980 & 0.1930 & 0.8154 & -3.086 & -3.083 & 0.9770 & 0.9840 \\
\hline unknown & -2.960 & -3.026 & 0.5300 & 0.6618 & -2.876 & -2.871 & 0.9550 & 0.9744 & -2.906 & -3.172 & 0.1070 & 0.5288 \\
\hline unknown & -4.362 & -4.407 & 0.4560 & 0.5921 & -4.299 & -4.311 & 0.9260 & 0.9664 & -4.347 & -4.434 & 0.4680 & 0.5671 \\
\hline unknown & -3.372 & -3.368 & 0.9610 & 0.9719 & -3.259 & -3.318 & 0.5010 & 0.8215 & -3.367 & -3.497 & 0.1600 & 0.5288 \\
\hline unknown & -4.942 & -4.935 & 0.9490 & 0.9665 & -4.845 & -5.065 & 0.1300 & 0.8154 & -5.120 & -5.137 & 0.7640 & 0.8164 \\
\hline unknown & -2.689 & -2.801 & 0.3170 & 0.5110 & -2.605 & -2.853 & 0.2740 & 0.8154 & -2.969 & -2.818 & 0.3010 & 0.5288 \\
\hline unknown & -3.433 & -3.395 & 0.8330 & 0.8781 & -3.400 & -3.428 & 0.8810 & 0.9543 & -3.349 & -3.634 & 0.0940 & 0.5288 \\
\hline unknown & -3.425 & -3.371 & 0.2270 & 0.5110 & -3.331 & -3.426 & 0.5100 & 0.8215 & -3.527 & -3.387 & 0.0540 & 0.5288 \\
\hline unknown & -3.259 & -3.364 & 0.3020 & 0.5110 & -3.219 & -3.368 & 0.2900 & 0.8154 & -3.371 & -3.484 & 0.2230 & 0.5288 \\
\hline unknown & -4.739 & -4.912 & 0.1770 & 0.5110 & -4.764 & -4.833 & 0.7000 & 0.8844 & -4.825 & -4.897 & 0.3920 & 0.5291 \\
\hline unknown & -5.711 & -5.871 & 0.0910 & 0.5110 & -5.616 & -5.704 & 0.4940 & 0.8215 & -5.642 & -5.801 & 0.2740 & 0.5288 \\
\hline unknown & -5.388 & -5.408 & 0.7310 & 0.8090 & -5.317 & -5.277 & 0.7880 & 0.9305 & -5.307 & -5.422 & 0.4090 & 0.5334 \\
\hline unknown & -4.688 & -4.720 & 0.7290 & 0.8081 & -4.583 & -4.588 & 0.9620 & 0.9768 & -4.685 & -4.922 & 0.1010 & 0.5288 \\
\hline unknown & -3.540 & -4.115 & 0.0440 & 0.5110 & -3.403 & -3.116 & 0.3560 & 0.8154 & -3.966 & -3.974 & 0.9840 & 0.9894 \\
\hline unknown & -3.856 & -3.816 & 0.6810 & 0.7817 & -3.627 & -3.801 & 0.1110 & 0.8154 & -3.859 & -3.885 & 0.6820 & 0.7470 \\
\hline unknown & -4.947 & -5.115 & 0.1220 & 0.5110 & -4.985 & -4.957 & 0.8030 & 0.9320 & -5.053 & -5.117 & 0.5040 & 0.5957 \\
\hline unknown & -3.624 & -3.713 & 0.4150 & 0.5667 & -3.480 & -3.821 & 0.1070 & 0.8154 & -3.910 & -3.755 & 0.2940 & 0.5288 \\
\hline unknown & -0.476 & -0.528 & 0.6430 & 0.7485 & -0.420 & -0.412 & 0.9360 & 0.9664 & -0.414 & -0.717 & 0.0280 & 0.5288 \\
\hline unknown & -4.465 & -4.573 & 0.4230 & 0.5705 & -4.558 & -4.476 & 0.3830 & 0.8154 & -4.450 & -4.785 & 0.1030 & 0.5288 \\
\hline unknown & -4.821 & -4.876 & 0.6170 & 0.7324 & -4.869 & -4.819 & 0.6630 & 0.8614 & -4.903 & -5.022 & 0.2240 & 0.5288 \\
\hline unknown & -3.916 & -4.041 & 0.2260 & 0.5110 & -3.900 & -3.867 & 0.7990 & 0.9305 & -3.830 & -4.220 & 0.0140 & 0.5288 \\
\hline unknown & -4.110 & -4.092 & 0.8000 & 0.8632 & -4.013 & -4.107 & 0.4770 & 0.8215 & -4.205 & -4.266 & 0.5510 & 0.6357 \\
\hline unknown & -4.276 & -4.325 & 0.6910 & 0.7868 & -4.230 & -4.228 & 0.9850 & 0.9887 & -4.328 & -4.499 & 0.1850 & 0.5288 \\
\hline unknown & -6.570 & -6.817 & 0.4610 & 0.5931 & -6.544 & -6.548 & 0.9890 & 0.9909 & -6.960 & -6.400 & 0.2170 & 0.5288 \\
\hline unknown & -2.394 & -2.434 & 0.7070 & 0.8002 & -2.268 & -2.412 & 0.1460 & 0.8154 & -2.432 & -2.480 & 0.3910 & 0.5291 \\
\hline unknown & -4.396 & -4.552 & 0.1440 & 0.5110 & -4.328 & -4.507 & 0.1550 & 0.8154 & -4.506 & -4.590 & 0.2190 & 0.5288 \\
\hline unknown & -4.577 & -4.672 & 0.5560 & 0.6815 & -4.412 & -4.544 & 0.3250 & 0.8154 & -4.605 & -4.613 & 0.9390 & 0.9492 \\
\hline unknown & -4.964 & -5.082 & 0.3920 & 0.5537 & -4.874 & -5.142 & 0.3120 & 0.8154 & -5.261 & -5.126 & 0.2760 & 0.5288 \\
\hline unknown & -2.164 & -2.218 & 0.5450 & 0.6722 & -2.055 & -2.170 & 0.3680 & 0.8154 & -2.204 & -2.358 & 0.0170 & 0.5288 \\
\hline unknown & 0.067 & -0.017 & 0.3730 & 0.5400 & 0.189 & 0.048 & 0.2230 & 0.8154 & 0.049 & -0.100 & 0.1420 & 0.5288 \\
\hline unknown & -6.515 & -6.991 & 0.4640 & 0.5962 & -6.428 & -6.599 & 0.7210 & 0.8935 & -7.251 & -6.202 & 0.1500 & 0.5288 \\
\hline unknown & -6.398 & -6.595 & 0.5390 & 0.6692 & -6.282 & -6.445 & 0.5260 & 0.8240 & -6.732 & -6.318 & 0.3010 & 0.5288 \\
\hline unknown & -4.842 & -5.362 & 0.3160 & 0.5110 & -4.819 & -4.787 & 0.8800 & 0.9543 & -5.413 & -4.626 & 0.1960 & 0.5288 \\
\hline unknown & -5.410 & -5.417 & 0.9200 & 0.9461 & -5.285 & -5.247 & 0.7540 & 0.9108 & -5.333 & -5.443 & 0.1450 & 0.5288 \\
\hline unknown & -2.858 & -2.883 & 0.8130 & 0.8667 & -2.764 & -2.824 & 0.4430 & 0.8154 & -2.886 & -3.014 & 0.0860 & 0.5288 \\
\hline unknown & -2.603 & -2.678 & 0.4400 & 0.5837 & -2.467 & -2.537 & 0.4290 & 0.8154 & -2.622 & -2.761 & 0.1700 & 0.5288 \\
\hline unknown & -5.552 & -5.574 & 0.8030 & 0.8632 & -5.413 & -5.532 & 0.4010 & 0.8154 & -5.713 & -5.507 & 0.2600 & 0.5288 \\
\hline unknown & -1.445 & -1.461 & 0.8200 & 0.8718 & -1.344 & -1.454 & 0.2300 & 0.8154 & -1.493 & -1.631 & 0.1650 & 0.5288 \\
\hline unknown & -4.407 & -4.330 & 0.3570 & 0.5297 & -4.193 & -4.425 & 0.1050 & 0.8154 & -4.407 & -4.510 & 0.1230 & 0.5288 \\
\hline unknown & -5.561 & -6.576 & 0.1810 & 0.5110 & -5.547 & -6.017 & 0.5230 & 0.8229 & -6.751 & -5.818 & 0.2850 & 0.5288 \\
\hline unknown & -4.440 & -4.503 & 0.5970 & 0.7135 & -4.287 & -4.418 & 0.1240 & 0.8154 & -4.516 & -4.448 & 0.7180 & 0.7789 \\
\hline unknown & -2.106 & -2.175 & 0.4210 & 0.5705 & -1.978 & -2.115 & 0.1270 & 0.8154 & -2.168 & -2.258 & 0.3190 & 0.5288 \\
\hline unknown & -3.634 & -3.799 & 0.1910 & 0.5110 & -3.644 & -3.735 & 0.5730 & 0.8315 & -3.798 & -3.721 & 0.4610 & 0.5658 \\
\hline unknown & -5.496 & -6.290 & 0.2560 & 0.5110 & -5.257 & -5.596 & 0.5370 & 0.8240 & -6.308 & -5.364 & 0.2830 & 0.5288 \\
\hline unknown & -5.549 & -6.069 & 0.2960 & 0.5110 & -5.387 & -5.331 & 0.8450 & 0.9439 & -6.357 & -5.246 & 0.1770 & 0.5288 \\
\hline unknown & -3.021 & -3.681 & 0.3100 & 0.5110 & -2.759 & -2.979 & 0.7000 & 0.8844 & -4.102 & -2.740 & 0.1730 & 0.5288 \\
\hline unknown & -0.799 & -0.845 & 0.4210 & 0.5705 & -0.706 & -0.854 & 0.2680 & 0.8154 & -0.884 & -0.784 & 0.0340 & 0.5288 \\
\hline unknown & -2.076 & -2.384 & 0.0830 & 0.5110 & -2.093 & -2.179 & 0.5600 & 0.8257 & -2.409 & -2.077 & 0.2270 & 0.5288 \\
\hline unknown & -4.285 & -4.447 & 0.2550 & 0.5110 & -4.231 & -4.332 & 0.5480 & 0.8240 & -4.537 & -4.173 & 0.0670 & 0.5288 \\
\hline unknown & -4.615 & -4.610 & 0.9600 & 0.9719 & -4.525 & -4.643 & 0.4320 & 0.8154 & -4.680 & -4.542 & 0.0700 & 0.5288 \\
\hline unknown & -5.749 & -5.913 & 0.1920 & 0.5110 & -5.767 & -5.744 & 0.8630 & 0.9524 & -6.017 & -5.681 & 0.0960 & 0.5288 \\
\hline unknown & -2.306 & -2.375 & 0.2740 & 0.5110 & -2.265 & -2.364 & 0.4370 & 0.8154 & -2.382 & -2.331 & 0.5350 & 0.6238 \\
\hline unknown & -5.915 & -6.065 & 0.2940 & 0.5110 & -5.801 & -5.949 & 0.4060 & 0.8154 & -6.003 & -5.761 & 0.2570 & 0.5288 \\
\hline unknown & -4.543 & -4.639 & 0.1030 & 0.5110 & -4.520 & -4.639 & 0.3620 & 0.8154 & -4.648 & -4.582 & 0.1940 & 0.5288 \\
\hline unknown & -3.126 & -3.151 & 0.8140 & 0.8667 & -3.027 & -3.161 & 0.1250 & 0.8154 & -3.146 & -3.371 & 0.1680 & 0.5288 \\
\hline unknown & -3.870 & -3.904 & 0.7770 & 0.8443 & -3.764 & -3.911 & 0.4370 & 0.8154 & -3.997 & -4.046 & 0.6420 & 0.7196 \\
\hline unknown & -3.654 & -3.577 & 0.6280 & 0.7385 & -3.632 & -3.307 & 0.1740 & 0.8154 & -3.335 & -3.496 & 0.6350 & 0.7143 \\
\hline unknown & -5.494 & -6.003 & 0.3870 & 0.5509 & -5.119 & -5.369 & 0.5000 & 0.8215 & -6.190 & -5.312 & 0.2520 & 0.5288 \\
\hline unknown & -5.651 & -5.909 & 0.4030 & 0.5584 & -5.496 & -5.489 & 0.9780 & 0.9853 & -5.811 & -5.471 & 0.3580 & 0.5291 \\
\hline unknown & -4.445 & -4.902 & 0.2400 & 0.5110 & -4.492 & -4.270 & 0.2890 & 0.8154 & -4.831 & -4.249 & 0.3100 & 0.5288 \\
\hline unknown & -1.997 & -1.878 & 0.5870 & 0.7066 & -1.688 & -1.952 & 0.0140 & 0.8154 & -1.919 & -2.100 & 0.2360 & 0.5288 \\
\hline unknown & -5.083 & -5.027 & 0.4370 & 0.5821 & -4.895 & -4.995 & 0.1510 & 0.8154 & -4.932 & -5.270 & 0.0420 & 0.5288 \\
\hline unknown & -2.201 & -2.250 & 0.5930 & 0.7112 & -2.102 & -2.198 & 0.3700 & 0.8154 & -2.231 & -2.328 & 0.3700 & 0.5291 \\
\hline unknown & -4.790 & -4.867 & 0.3590 & 0.5297 & -4.753 & -4.649 & 0.4990 & 0.8215 & -4.739 & -4.999 & 0.0180 & 0.5288 \\
\hline unknown & -4.378 & -4.479 & 0.3770 & 0.5406 & -4.375 & -4.514 & 0.2860 & 0.8154 & -4.643 & -4.633 & 0.8670 & 0.9007 \\
\hline unknown & -5.579 & -6.501 & 0.2350 & 0.5110 & -5.247 & -5.826 & 0.4650 & 0.8202 & -6.882 & -5.602 & 0.2820 & 0.5288 \\
\hline unknown & -2.540 & -3.303 & 0.2860 & 0.5110 & -2.231 & -2.575 & 0.5210 & 0.8229 & -3.897 & -2.529 & 0.2160 & 0.5288 \\
\hline unknown & -5.805 & -6.442 & 0.2160 & 0.5110 & -5.376 & -5.848 & 0.2900 & 0.8154 & -6.903 & -5.561 & 0.1740 & 0.5288 \\
\hline unknown & -2.332 & -2.387 & 0.5240 & 0.6572 & -2.350 & -2.345 & 0.9570 & 0.9749 & -2.365 & -2.583 & 0.0930 & 0.5288 \\
\hline unknown & -4.830 & -5.669 & 0.3060 & 0.5110 & -4.531 & -4.968 & 0.5410 & 0.8240 & -5.806 & -4.756 & 0.2840 & 0.5288 \\
\hline unknown & -4.527 & -5.184 & 0.2510 & 0.5110 & -4.285 & -4.550 & 0.4990 & 0.8215 & -5.060 & -4.391 & 0.2990 & 0.5288 \\
\hline $\mathrm{vn}$ & -2.081 & -2.840 & 0.2950 & 0.5110 & -1.802 & -2.065 & 0.6080 & 0.8410 & -3.161 & -2.009 & 0.2400 & 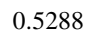 \\
\hline
\end{tabular}




\begin{tabular}{|c|c|c|c|c|c|c|c|c|c|c|c|c|}
\hline unknown & -0.515 & -1.215 & 0.2420 & 0.5110 & -0.275 & -0.532 & 0.5700 & 0.8315 & -1.791 & -0.360 & 0.1890 & 0.5288 \\
\hline unknown & -3.395 & -3.975 & 0.2270 & 0.5110 & -3.258 & -3.302 & 0.9320 & 0.9664 & -4.256 & -3.175 & 0.1860 & 0.5288 \\
\hline unknown & -5.450 & -6.175 & 0.2430 & 0.5110 & -5.188 & -4.900 & 0.5580 & 0.8240 & -6.700 & -5.007 & 0.2250 & 0.5288 \\
\hline unknown & -4.010 & -4.701 & 0.2550 & 0.5110 & -3.835 & -3.938 & 0.8420 & 0.9427 & -4.871 & -3.671 & 0.1780 & 0.5288 \\
\hline unknown & -0.785 & -1.513 & 0.2190 & 0.5110 & -0.754 & -0.691 & 0.8550 & 0.9474 & -1.812 & -0.587 & 0.1940 & 0.5288 \\
\hline unknown & -4.235 & -4.765 & 0.2230 & 0.5110 & -4.258 & -4.130 & 0.5800 & 0.8317 & -4.671 & -4.114 & 0.3190 & 0.5288 \\
\hline unknown & -5.749 & -6.666 & 0.3140 & 0.5110 & -5.042 & -4.889 & 0.8160 & 0.9335 & -7.164 & -5.225 & 0.2100 & 0.5288 \\
\hline unknown & -5.693 & -6.077 & 0.3380 & 0.5293 & -5.649 & -5.560 & 0.7350 & 0.8997 & -6.151 & -5.606 & 0.3160 & 0.5288 \\
\hline unknown & -3.010 & -3.036 & 0.7810 & 0.8469 & -2.938 & -3.057 & 0.2310 & 0.8154 & -3.073 & -3.196 & 0.2270 & 0.5288 \\
\hline unknown & -5.142 & -5.799 & 0.3340 & 0.5286 & -4.847 & -5.301 & 0.3710 & 0.8154 & -6.217 & -5.093 & 0.2680 & 0.5288 \\
\hline unknown & -4.548 & -4.607 & 0.6530 & 0.7581 & -4.421 & -4.505 & 0.6280 & 0.8537 & -4.631 & -4.777 & 0.3200 & 0.5288 \\
\hline unknown & -4.988 & -5.482 & 0.2660 & 0.5110 & -4.751 & -4.943 & 0.6380 & 0.8537 & -5.208 & -4.844 & 0.4670 & 0.5671 \\
\hline unknown & -3.310 & -3.866 & 0.3120 & 0.5110 & -2.938 & -3.148 & 0.5150 & 0.8226 & -4.017 & -3.125 & 0.2990 & 0.5288 \\
\hline unknown & -5.927 & -6.494 & 0.1760 & 0.5110 & -5.748 & -5.616 & 0.5900 & 0.8339 & -6.309 & -5.764 & 0.3750 & 0.5291 \\
\hline unknown & -3.047 & -3.171 & 0.2480 & 0.5110 & -2.997 & -3.120 & 0.2950 & 0.8154 & -3.111 & -3.260 & 0.0560 & 0.5288 \\
\hline unknown & -4.290 & -4.369 & 0.4720 & 0.6035 & -4.131 & -4.242 & 0.2700 & 0.8154 & -4.393 & -4.410 & 0.8490 & 0.8881 \\
\hline unknown & -5.924 & -6.356 & 0.2680 & 0.5110 & -5.804 & -5.800 & 0.9750 & 0.9845 & -6.318 & -5.764 & 0.2200 & 0.5288 \\
\hline unknown & -4.292 & -4.681 & 0.2530 & 0.5110 & -4.061 & -4.101 & 0.8740 & 0.9543 & -4.481 & -4.057 & 0.3970 & 0.5291 \\
\hline unknown & -2.968 & -3.466 & 0.2690 & 0.5110 & -2.869 & -2.685 & 0.4930 & 0.8215 & -3.561 & -2.777 & 0.2660 & 0.5288 \\
\hline unknown & -5.282 & -5.762 & 0.2040 & 0.5110 & -5.160 & -5.092 & 0.8100 & 0.9332 & -5.711 & -5.139 & 0.4110 & 0.5340 \\
\hline unknown & -4.295 & -4.824 & 0.2500 & 0.5110 & -4.267 & -4.032 & 0.1660 & 0.8154 & -4.824 & -4.219 & 0.3800 & 0.5291 \\
\hline unknown & -7.292 & -8.077 & 0.2840 & 0.5110 & -6.838 & -7.365 & 0.3970 & 0.8154 & -8.188 & -7.412 & 0.3820 & 0.5291 \\
\hline unknown & -4.337 & -5.158 & 0.2800 & 0.5110 & -4.070 & -4.466 & 0.5100 & 0.8215 & -5.830 & -4.341 & 0.2240 & 0.5288 \\
\hline unknown & -0.493 & -1.162 & 0.2870 & 0.5110 & -0.189 & -0.498 & 0.4610 & 0.8202 & -1.800 & -0.363 & 0.1960 & 0.5288 \\
\hline unknown & -3.840 & -4.396 & 0.2370 & 0.5110 & -3.583 & -3.993 & 0.1990 & 0.8154 & -4.814 & -3.702 & 0.2200 & 0.5288 \\
\hline unknown & -2.590 & -3.520 & 0.2200 & 0.5110 & -2.095 & -2.694 & 0.3910 & 0.8154 & -3.785 & -2.558 & 0.2880 & 0.5288 \\
\hline unknown & -4.719 & -5.426 & 0.1820 & 0.5110 & -4.343 & -4.840 & 0.3900 & 0.8154 & -5.145 & -4.789 & 0.6090 & 0.6874 \\
\hline unknown & -5.537 & -6.209 & 0.4020 & 0.5584 & -5.503 & -4.717 & 0.0100 & 0.8154 & -7.082 & -4.919 & 0.1730 & 0.5288 \\
\hline unknown & -3.326 & -3.346 & 0.8430 & 0.8859 & -3.271 & -3.357 & 0.4030 & 0.8154 & -3.406 & -3.473 & 0.4020 & 0.5307 \\
\hline unknown & -3.855 & -3.886 & 0.5820 & 0.7029 & -3.788 & -3.756 & 0.7270 & 0.8936 & -3.822 & -3.858 & 0.7210 & 0.7798 \\
\hline unknown & -2.471 & -3.252 & 0.2390 & 0.5110 & -2.214 & -2.483 & 0.5620 & 0.8263 & -3.717 & -2.337 & 0.1920 & 0.5288 \\
\hline unknown & -3.189 & -3.824 & 0.2400 & 0.5110 & -2.947 & -3.132 & 0.5700 & 0.8315 & -3.731 & -3.058 & 0.3400 & 0.5291 \\
\hline unknown & -2.306 & -2.915 & 0.1970 & 0.5110 & -2.085 & -2.228 & 0.6710 & 0.8614 & -3.221 & -2.115 & 0.2010 & 0.5288 \\
\hline unknown & 0.277 & -0.419 & 0.2180 & 0.5110 & 0.495 & 0.519 & 0.9360 & 0.9664 & -0.699 & 0.449 & 0.2400 & 0.5288 \\
\hline unknown & -5.077 & -5.298 & 0.6320 & 0.7405 & -5.076 & -5.020 & 0.8980 & 0.9601 & -6.368 & -5.123 & 0.2350 & 0.5288 \\
\hline unknown & -4.622 & -5.210 & 0.2740 & 0.5110 & -4.367 & -4.488 & 0.7040 & 0.8844 & -5.090 & -4.395 & 0.3210 & 0.5288 \\
\hline unknown & -1.022 & -1.659 & 0.2510 & 0.5110 & -0.781 & -0.990 & 0.5840 & 0.8339 & -2.062 & -0.814 & 0.1780 & 0.5288 \\
\hline unknown & -1.626 & -2.328 & 0.2410 & 0.5110 & -1.342 & -1.310 & 0.9330 & 0.9664 & -2.402 & -1.368 & 0.2100 & 0.5288 \\
\hline unknown & -2.835 & -3.422 & 0.3090 & 0.5110 & -2.790 & -2.736 & 0.9060 & 0.9647 & -3.838 & -2.687 & 0.1890 & 0.5288 \\
\hline unknown & -3.820 & -4.391 & 0.1950 & 0.5110 & -3.727 & -3.694 & 0.8830 & 0.9543 & -4.420 & -3.758 & 0.2680 & 0.5288 \\
\hline unknown & -0.388 & -1.125 & 0.1930 & 0.5110 & -0.176 & -0.213 & 0.9000 & 0.9601 & -1.464 & -0.186 & 0.1690 & 0.5288 \\
\hline unknown & -2.835 & -3.571 & 0.1930 & 0.5110 & -2.493 & -2.787 & 0.5880 & 0.8339 & -3.707 & -2.549 & 0.2100 & 0.5288 \\
\hline unknown & -5.286 & -5.985 & 0.3740 & 0.5400 & -4.685 & -5.033 & 0.5360 & 0.8240 & -6.416 & -5.127 & 0.2750 & 0.5288 \\
\hline unknown & -5.293 & -5.833 & 0.2420 & 0.5110 & -5.164 & -5.122 & 0.8820 & 0.9543 & -5.656 & -5.047 & 0.2630 & 0.5288 \\
\hline unknown & -2.127 & -2.655 & 0.2310 & 0.5110 & -1.377 & -2.130 & 0.2200 & 0.8154 & -2.877 & -1.724 & 0.1570 & 0.5288 \\
\hline unknown & 2.224 & 2.171 & 0.5510 & 0.6774 & 2.313 & 2.118 & 0.2260 & 0.8154 & 2.021 & 2.210 & 0.2300 & 0.5288 \\
\hline unknown & -4.274 & -4.702 & 0.1710 & 0.5110 & -4.204 & -4.424 & 0.4270 & 0.8154 & -4.282 & -4.880 & 0.0790 & 0.5288 \\
\hline unknown & -2.208 & -2.239 & 0.7500 & 0.8214 & -2.087 & -2.201 & 0.2400 & 0.8154 & -2.211 & -2.363 & 0.0090 & 0.5288 \\
\hline unknown & -3.805 & -4.583 & 0.2470 & 0.5110 & -3.423 & -3.826 & 0.3750 & 0.8154 & -4.832 & -3.708 & 0.2800 & 0.5288 \\
\hline unknown & -5.907 & -6.458 & 0.4380 & 0.5826 & -5.300 & -5.842 & 0.4330 & 0.8154 & -6.437 & -5.749 & 0.4240 & 0.5434 \\
\hline unknown & -6.373 & -7.082 & 0.1860 & 0.5110 & -6.074 & -6.261 & 0.5530 & 0.8240 & -6.850 & -6.225 & 0.4120 & 0.5340 \\
\hline unknown & -1.442 & -2.140 & 0.2570 & 0.5110 & -1.213 & -1.377 & 0.5530 & 0.8240 & -2.577 & -1.375 & 0.2780 & 0.5288 \\
\hline unknown & -5.081 & -5.798 & 0.2360 & 0.5110 & -4.774 & -5.171 & 0.3380 & 0.8154 & -6.028 & -5.062 & 0.2550 & 0.5288 \\
\hline unknown & -3.294 & -4.016 & 0.2410 & 0.5110 & -3.014 & -3.194 & 0.5450 & 0.8240 & -4.100 & -3.187 & 0.3020 & 0.5288 \\
\hline unknown & -4.206 & -4.942 & 0.2250 & 0.5110 & -3.995 & -4.079 & 0.8110 & 0.9332 & -5.034 & -4.039 & 0.3250 & 0.5288 \\
\hline unknown & -5.284 & -5.305 & 0.8400 & 0.8840 & -5.288 & -5.216 & 0.6010 & 0.8391 & -5.376 & -5.392 & 0.9160 & 0.9362 \\
\hline unknown & -4.004 & -4.772 & 0.1170 & 0.5110 & -3.851 & -3.975 & 0.6550 & 0.8614 & -4.730 & -4.067 & 0.3660 & 0.5291 \\
\hline unknown & -3.476 & -4.373 & 0.1740 & 0.5110 & -3.190 & -3.146 & 0.8940 & 0.9595 & -3.881 & -3.127 & 0.3820 & 0.5291 \\
\hline unknown & -3.143 & -3.852 & 0.2210 & 0.5110 & -2.949 & -3.034 & 0.7970 & 0.9305 & -3.881 & -3.073 & 0.3550 & 0.5291 \\
\hline unknown & -5.641 & -6.383 & 0.1010 & 0.5110 & -5.354 & -5.696 & 0.4320 & 0.8154 & -6.166 & -5.540 & 0.4070 & 0.5321 \\
\hline unknown & -6.490 & -7.095 & 0.0540 & 0.5110 & -6.209 & -6.503 & 0.2340 & 0.8154 & -6.770 & -6.251 & 0.4050 & 0.5307 \\
\hline unknown & -4.713 & -5.392 & 0.3120 & 0.5110 & -4.417 & -4.820 & 0.3170 & 0.8154 & -5.505 & -4.598 & 0.2680 & 0.5288 \\
\hline unknown & -6.660 & -7.125 & 0.1790 & 0.5110 & -6.421 & -6.726 & 0.4410 & 0.8154 & -7.322 & -6.634 & 0.3440 & 0.5291 \\
\hline unknown & -3.140 & -3.931 & 0.2160 & 0.5110 & -2.972 & -3.303 & 0.4050 & 0.8154 & -4.389 & -3.185 & 0.2370 & 0.5288 \\
\hline unknown & -5.252 & -6.061 & 0.2550 & 0.5110 & -4.846 & -5.370 & 0.4210 & 0.8154 & -6.250 & -5.256 & 0.3150 & 0.5288 \\
\hline unknown & 0.080 & -0.542 & 0.2530 & 0.5110 & 0.544 & 0.019 & 0.2760 & 0.8154 & -0.904 & 0.163 & 0.2810 & 0.5288 \\
\hline unknown & -3.706 & -4.591 & 0.2590 & 0.5110 & -3.188 & -3.891 & 0.2720 & 0.8154 & -4.836 & -3.727 & 0.3160 & 0.5288 \\
\hline unknown & -1.553 & -2.289 & 0.2890 & 0.5110 & -1.073 & -1.759 & 0.2590 & 0.8154 & -2.693 & -1.588 & 0.3180 & 0.5288 \\
\hline unknown & -3.107 & -3.591 & 0.1570 & 0.5110 & -2.692 & -3.090 & 0.1690 & 0.8154 & -3.828 & -2.937 & 0.2720 & 0.5288 \\
\hline unknown & -5.009 & -4.924 & 0.8580 & 0.8943 & -4.567 & -4.686 & 0.6490 & 0.8614 & -7.041 & -4.797 & 0.0910 & 0.5288 \\
\hline unknown & -6.714 & -8.138 & 0.1580 & 0.5110 & -6.320 & -6.967 & 0.1810 & 0.8154 & -7.919 & -6.636 & 0.3820 & 0.5291 \\
\hline unknown & -1.677 & -1.715 & 0.7160 & 0.8053 & -1.609 & -1.682 & 0.3650 & 0.8154 & -1.679 & -1.848 & 0.0590 & 0.5288 \\
\hline unknown & -5.836 & -5.956 & 0.1320 & 0.5110 & -5.723 & -5.717 & 0.9360 & 0.9664 & -5.797 & -5.832 & 0.7800 & 0.8321 \\
\hline unknown & -1.265 & -1.957 & 0.2550 & 0.5110 & -1.093 & -1.422 & 0.4360 & 0.8154 & -2.615 & -1.296 & 0.2100 & 0.5288 \\
\hline unknown & -3.924 & -4.311 & 0.5190 & 0.6526 & -4.030 & -3.816 & 0.6030 & 0.8391 & -5.034 & -3.599 & 0.0970 & 0.5288 \\
\hline unknown & -2.090 & -2.733 & 0.2680 & 0.5110 & -1.834 & -2.005 & 0.5030 & 0.8215 & -3.061 & -1.999 & 0.2710 & 0.5288 \\
\hline $\mathrm{vn}$ & -0.738 & -1.582 & 0.1660 & 0.5110 & -0.508 & -0.627 & 0.7740 & 0.9234 & -1.844 & -0.558 & 0.2200 & . \\
\hline
\end{tabular}




\begin{tabular}{|c|c|c|c|c|c|c|c|c|c|c|c|c|}
\hline unknown & 1.721 & 1.207 & 0.2060 & 0.5110 & 1.878 & 1.679 & 0.3770 & 0.8154 & 0.663 & 1.780 & 0.2190 & 0.5288 \\
\hline unknown & -5.303 & -5.304 & 0.9990 & 0.9988 & -4.784 & -4.660 & 0.7220 & 0.8935 & -6.606 & -4.728 & 0.1380 & 0.5288 \\
\hline unknown & -5.278 & -5.677 & 0.5160 & 0.6503 & -5.361 & -5.159 & 0.6390 & 0.8537 & -6.405 & -4.896 & 0.0770 & 0.5288 \\
\hline unknown & -5.313 & -5.311 & 0.9880 & 0.9932 & -5.167 & -5.356 & 0.2010 & 0.8154 & -5.276 & -5.398 & 0.3280 & 0.5288 \\
\hline unknown & -1.596 & -2.134 & 0.2190 & 0.5110 & -1.193 & -1.565 & 0.1810 & 0.8154 & -2.724 & -1.466 & 0.1710 & 0.5288 \\
\hline unknown & -3.196 & -4.073 & 0.1400 & 0.5110 & -3.350 & -3.121 & 0.5040 & 0.8215 & -4.120 & -3.040 & 0.2360 & 0.5288 \\
\hline unknown & -1.112 & -1.170 & 0.3740 & 0.5400 & -1.053 & -1.188 & 0.2970 & 0.8154 & -1.226 & -1.198 & 0.6330 & 0.7138 \\
\hline unknown & 1.239 & 1.127 & 0.1410 & 0.5110 & 1.249 & 1.296 & 0.6500 & 0.8614 & 1.214 & 1.109 & 0.2850 & 0.5288 \\
\hline unknown & -2.241 & -2.974 & 0.1970 & 0.5110 & -1.932 & -2.147 & 0.6980 & 0.8844 & -3.049 & -1.917 & 0.2000 & 0.5288 \\
\hline unknown & -0.680 & -0.680 & 0.9980 & 0.9988 & -0.540 & -0.635 & 0.2230 & 0.8154 & -0.642 & -0.714 & 0.3970 & 0.5291 \\
\hline unknown & -3.663 & -4.437 & 0.1460 & 0.5110 & -3.372 & -3.817 & 0.4780 & 0.8215 & -4.365 & -3.387 & 0.2810 & 0.5288 \\
\hline unknown & 0.370 & 0.304 & 0.5080 & 0.6425 & 0.457 & 0.353 & 0.4600 & 0.8202 & 0.307 & 0.301 & 0.9370 & 0.9492 \\
\hline unknown & -4.587 & -4.540 & 0.6130 & 0.7283 & -4.406 & -4.500 & 0.5330 & 0.8240 & -4.607 & -4.451 & 0.0270 & 0.5288 \\
\hline unknown & -3.532 & -4.404 & 0.1200 & 0.5110 & -3.605 & -3.301 & 0.6010 & 0.8391 & -4.567 & -3.640 & 0.3180 & 0.5288 \\
\hline unknown & -3.514 & -4.264 & 0.2030 & 0.5110 & -3.262 & -3.499 & 0.6710 & 0.8614 & -4.360 & -3.202 & 0.1820 & 0.5288 \\
\hline unknown & -4.882 & -5.614 & 0.1440 & 0.5110 & -4.605 & -4.983 & 0.4950 & 0.8215 & -5.611 & -4.622 & 0.2600 & 0.5288 \\
\hline unknown & -5.639 & -6.309 & 0.2030 & 0.5110 & -5.268 & -5.555 & 0.5850 & 0.8339 & -6.215 & -5.420 & 0.3550 & 0.5291 \\
\hline unknown & -5.729 & -6.636 & 0.2090 & 0.5110 & -5.539 & -5.887 & 0.2950 & 0.8154 & -6.798 & -5.928 & 0.3880 & 0.5291 \\
\hline unknown & -2.445 & -3.148 & 0.2500 & 0.5110 & -1.983 & -2.475 & 0.2010 & 0.8154 & -3.524 & -2.352 & 0.2820 & 0.5288 \\
\hline unknown & -4.465 & -5.176 & 0.2950 & 0.5110 & -3.892 & -4.568 & 0.2370 & 0.8154 & -5.500 & -4.434 & 0.3280 & 0.5288 \\
\hline unknown & -6.208 & -7.245 & 0.1280 & 0.5110 & -5.878 & -6.455 & 0.3080 & 0.8154 & -7.290 & -6.582 & 0.4630 & 0.5661 \\
\hline unknown & -4.681 & -5.400 & 0.2090 & 0.5110 & -4.255 & -4.698 & 0.2470 & 0.8154 & -5.690 & -4.529 & 0.2820 & 0.5288 \\
\hline unknown & -3.916 & -4.008 & 0.3530 & 0.5297 & -3.781 & -3.922 & 0.2960 & 0.8154 & -3.951 & -4.009 & 0.1220 & 0.5288 \\
\hline unknown & -3.735 & -4.388 & 0.2340 & 0.5110 & -3.382 & -3.721 & 0.3910 & 0.8154 & -4.692 & -3.607 & 0.2760 & 0.5288 \\
\hline unknown & -2.370 & -3.033 & 0.2970 & 0.5110 & -1.995 & -2.350 & 0.1260 & 0.8154 & -3.373 & -2.342 & 0.3330 & 0.5288 \\
\hline unknown & -2.170 & -2.999 & 0.1800 & 0.5110 & -1.856 & -2.146 & 0.2420 & 0.8154 & -3.249 & -2.068 & 0.2770 & 0.5288 \\
\hline unknown & -4.911 & -5.903 & 0.1270 & 0.5110 & -4.565 & -4.863 & 0.3350 & 0.8154 & -5.840 & -4.818 & 0.3510 & 0.5291 \\
\hline unknown & -4.801 & -5.608 & 0.1760 & 0.5110 & -4.475 & -4.929 & 0.4370 & 0.8154 & -5.728 & -4.632 & 0.3370 & 0.5288 \\
\hline unknown & -3.614 & -4.365 & 0.2430 & 0.5110 & -3.147 & -3.622 & 0.1230 & 0.8154 & -4.400 & -3.600 & 0.4050 & 0.5307 \\
\hline unknown & -5.134 & -5.801 & 0.2250 & 0.5110 & -4.831 & -5.085 & 0.4270 & 0.8154 & -6.143 & -5.062 & 0.2730 & 0.5288 \\
\hline unknown & -4.950 & -5.653 & 0.2810 & 0.5110 & -4.589 & -4.982 & 0.4200 & 0.8154 & -5.605 & -4.779 & 0.3940 & 0.5291 \\
\hline unknown & -5.053 & -5.971 & 0.1340 & 0.5110 & -4.613 & -5.093 & 0.1750 & 0.8154 & -5.851 & -4.943 & 0.3600 & 0.5291 \\
\hline unknown & -6.173 & -6.436 & 0.2020 & 0.5110 & -5.949 & -6.421 & 0.1270 & 0.8154 & -6.572 & -6.277 & 0.2650 & 0.5288 \\
\hline unknown & -5.134 & -5.247 & 0.2610 & 0.5110 & -5.075 & -5.027 & 0.6340 & 0.8537 & -5.435 & -5.363 & 0.4560 & 0.5647 \\
\hline unknown & -6.195 & -7.057 & 0.1390 & 0.5110 & -6.030 & -6.457 & 0.4700 & 0.8210 & -6.998 & -6.239 & 0.3680 & 0.5291 \\
\hline unknown & -3.756 & -4.534 & 0.2450 & 0.5110 & -3.400 & -3.872 & 0.3060 & 0.8154 & -4.634 & -3.853 & 0.3750 & 0.5291 \\
\hline unknown & -1.726 & -2.419 & 0.2370 & 0.5110 & -1.444 & -1.840 & 0.1590 & 0.8154 & -2.709 & -1.733 & 0.2700 & 0.5288 \\
\hline unknown & -5.987 & -6.860 & 0.1830 & 0.5110 & -5.689 & -6.264 & 0.3030 & 0.8154 & -6.715 & -6.094 & 0.4390 & 0.5519 \\
\hline unknown & -4.429 & -5.093 & 0.1510 & 0.5110 & -4.270 & -4.595 & 0.1800 & 0.8154 & -5.232 & -4.410 & 0.3100 & 0.5288 \\
\hline unknown & -7.355 & -8.395 & 0.3530 & 0.5297 & -6.631 & -7.335 & 0.0530 & 0.8154 & -8.192 & -6.989 & 0.3660 & 0.5291 \\
\hline unknown & -2.539 & -2.546 & 0.9310 & 0.9542 & -2.462 & -2.597 & 0.2330 & 0.8154 & -2.555 & -2.706 & 0.0000 & 0.1090 \\
\hline unknown & -0.772 & -1.599 & 0.1110 & 0.5110 & -0.532 & -1.080 & 0.1240 & 0.8154 & -1.896 & -0.793 & 0.2880 & 0.5288 \\
\hline unknown & -3.246 & -3.950 & 0.2490 & 0.5110 & -2.736 & -3.378 & 0.1730 & 0.8154 & -4.015 & -3.194 & 0.3780 & 0.5291 \\
\hline unknown & 0.977 & 0.388 & 0.2330 & 0.5110 & 1.367 & 0.994 & 0.3120 & 0.8154 & 0.015 & 1.058 & 0.2590 & 0.5288 \\
\hline unknown & -1.104 & -1.821 & 0.2790 & 0.5110 & -0.618 & -1.196 & 0.2100 & 0.8154 & -2.127 & -1.030 & 0.3210 & 0.5288 \\
\hline unknown & -3.030 & -3.875 & 0.1970 & 0.5110 & -2.615 & -3.233 & 0.2090 & 0.8154 & -4.220 & -3.078 & 0.3030 & 0.5288 \\
\hline unknown & -1.604 & -2.001 & 0.4270 & 0.5747 & -1.262 & -1.553 & 0.2650 & 0.8154 & -2.514 & -1.296 & 0.1930 & 0.5288 \\
\hline unknown & -4.036 & -4.677 & 0.1230 & 0.5110 & -3.715 & -4.048 & 0.3590 & 0.8154 & -5.045 & -3.939 & 0.2210 & 0.5288 \\
\hline unknown & -5.742 & -6.279 & 0.3360 & 0.5293 & -5.890 & -5.859 & 0.9220 & 0.9664 & -6.672 & -5.661 & 0.2010 & 0.5288 \\
\hline unknown & -4.182 & -5.001 & 0.1330 & 0.5110 & -3.880 & -4.174 & 0.2390 & 0.8154 & -5.012 & -4.086 & 0.3230 & 0.5288 \\
\hline unknown & -0.637 & -1.513 & 0.1550 & 0.5110 & -0.448 & -0.563 & 0.7750 & 0.9234 & -1.774 & -0.499 & 0.2290 & 0.5288 \\
\hline unknown & 0.176 & -0.683 & 0.1490 & 0.5110 & 0.503 & 0.030 & 0.1760 & 0.8154 & -0.889 & 0.151 & 0.3350 & 0.5288 \\
\hline unknown & -2.031 & -3.041 & 0.1140 & 0.5110 & -1.762 & -2.170 & 0.4750 & 0.8215 & -2.911 & -1.889 & 0.3710 & 0.5291 \\
\hline unknown & -5.587 & -6.129 & 0.2110 & 0.5110 & -5.390 & -5.606 & 0.4560 & 0.8202 & -6.167 & -5.277 & 0.2660 & 0.5288 \\
\hline unknown & -1.234 & -2.317 & 0.0870 & 0.5110 & -1.020 & -1.722 & 0.1710 & 0.8154 & -2.476 & -1.431 & 0.3610 & 0.5291 \\
\hline unknown & -2.457 & -2.621 & 0.0570 & 0.5110 & -2.432 & -2.543 & 0.4640 & 0.8202 & -2.659 & -2.630 & 0.7850 & 0.8358 \\
\hline unknown & -3.280 & -4.417 & 0.0480 & 0.5110 & -3.191 & -3.314 & 0.7900 & 0.9305 & -4.622 & -3.442 & 0.2550 & 0.5288 \\
\hline unknown & -5.683 & -6.345 & 0.0630 & 0.5110 & -5.362 & -5.944 & 0.1700 & 0.8154 & -6.431 & -5.668 & 0.2920 & 0.5288 \\
\hline unknown & -4.627 & -5.343 & 0.1910 & 0.5110 & -4.363 & -4.752 & 0.5430 & 0.8240 & -5.323 & -4.290 & 0.2690 & 0.5288 \\
\hline unknown & -5.822 & -6.594 & 0.0470 & 0.5110 & -5.599 & -6.053 & 0.4280 & 0.8154 & -6.142 & -5.607 & 0.5240 & 0.6120 \\
\hline unknown & -5.458 & -6.360 & 0.1710 & 0.5110 & -5.492 & -5.728 & 0.5560 & 0.8240 & -6.482 & -5.293 & 0.2430 & 0.5288 \\
\hline unknown & -4.764 & -5.073 & 0.6760 & 0.7797 & -4.901 & -6.057 & 0.0550 & 0.8154 & -4.515 & -3.987 & 0.4810 & 0.5800 \\
\hline unknown & -6.991 & -7.588 & 0.2090 & 0.5110 & -6.603 & -7.188 & 0.2440 & 0.8154 & -7.896 & -6.980 & 0.2780 & 0.5288 \\
\hline unknown & -5.584 & -6.337 & 0.3420 & 0.5293 & -5.162 & -5.631 & 0.3180 & 0.8154 & -6.399 & -5.637 & 0.4570 & 0.5647 \\
\hline unknown & -6.803 & -7.499 & 0.3460 & 0.5293 & -6.476 & -6.927 & 0.0650 & 0.8154 & -7.451 & -6.694 & 0.4350 & 0.5516 \\
\hline unknown & -5.354 & -6.102 & 0.2650 & 0.5110 & -5.060 & -5.313 & 0.3250 & 0.8154 & -6.374 & -5.120 & 0.2380 & 0.5288 \\
\hline unknown & -5.888 & -5.910 & 0.8840 & 0.9141 & -5.651 & -6.039 & 0.0970 & 0.8154 & -6.208 & -5.789 & 0.0350 & 0.5288 \\
\hline unknown & -5.827 & -6.641 & 0.1760 & 0.5110 & -5.651 & -5.900 & 0.4160 & 0.8154 & -6.644 & -5.724 & 0.3710 & 0.5291 \\
\hline unknown & -1.240 & -1.272 & 0.7390 & 0.8130 & -1.164 & -1.271 & 0.3670 & 0.8154 & -1.276 & -1.369 & 0.0950 & 0.5288 \\
\hline unknown & -4.705 & -5.638 & 0.2310 & 0.5110 & -4.397 & -4.931 & 0.2130 & 0.8154 & -5.765 & -4.810 & 0.3450 & 0.5291 \\
\hline unknown & -6.899 & -7.985 & 0.1320 & 0.5110 & -6.821 & -7.061 & 0.5460 & 0.8240 & -7.869 & -6.990 & 0.3710 & 0.5291 \\
\hline unknown & -6.924 & -7.563 & 0.3060 & 0.5110 & -6.731 & -7.028 & 0.4720 & 0.8211 & -7.645 & -7.008 & 0.3880 & 0.5291 \\
\hline unknown & -6.148 & -6.108 & 0.7070 & 0.8002 & -5.898 & -6.501 & 0.1120 & 0.8154 & -6.578 & -5.885 & 0.0770 & 0.5288 \\
\hline unknown & -5.828 & -6.592 & 0.2460 & 0.5110 & -5.381 & -6.050 & 0.0350 & 0.8154 & -6.330 & -5.925 & 0.5740 & 0.6580 \\
\hline unknown & -5.907 & -6.638 & 0.2200 & 0.5110 & -5.661 & -6.132 & 0.2320 & 0.8154 & -6.674 & -5.847 & 0.3900 & 0.5291 \\
\hline unknown & -5.709 & -6.257 & 0.4330 & 0.5795 & -5.412 & -5.821 & 0.2320 & 0.8154 & -6.427 & -5.595 & 0.3720 & 0.5291 \\
\hline un & -5.619 & 748 & 0.1440 & 0.5110 & -5.396 & -5.528 & 0.1180 & 0.8154 & -5.647 & -5.688 & 0.6850 & $0.74^{7}$ \\
\hline
\end{tabular}




\begin{tabular}{|c|c|c|c|c|c|c|c|c|c|c|c|c|}
\hline unknown & -4.576 & -4.671 & 0.3100 & 0.5110 & -4.521 & -4.722 & 0.1760 & 0.8154 & -4.744 & -4.614 & 0.2650 & 0.5288 \\
\hline unknown & -4.941 & -5.011 & 0.5940 & 0.7114 & -4.868 & -4.874 & 0.9670 & 0.9774 & -5.013 & -5.054 & 0.6510 & 0.7263 \\
\hline unknown & -3.385 & -3.476 & 0.2980 & 0.5110 & -3.380 & -3.461 & 0.5500 & 0.8240 & -3.488 & -3.568 & 0.2610 & 0.5288 \\
\hline unknown & -1.133 & -1.205 & 0.4490 & 0.5906 & -1.060 & -1.197 & 0.1430 & 0.8154 & -1.195 & -1.303 & 0.2390 & 0.5288 \\
\hline unknown & -2.498 & -2.573 & 0.4580 & 0.5922 & -2.434 & -2.569 & 0.0440 & 0.8154 & -2.572 & -2.673 & 0.2680 & 0.5288 \\
\hline unknown & -5.077 & -5.318 & 0.1110 & 0.5110 & -5.146 & -5.075 & 0.6970 & 0.8844 & -5.187 & -5.318 & 0.4490 & 0.5607 \\
\hline unknown & -2.108 & -2.210 & 0.3050 & 0.5110 & -2.057 & -2.145 & 0.2800 & 0.8154 & -2.139 & -2.273 & 0.2130 & 0.5288 \\
\hline unknown & -4.932 & -4.908 & 0.6880 & 0.7868 & -4.825 & -4.611 & 0.3060 & 0.8154 & -4.490 & -4.950 & 0.1440 & 0.5288 \\
\hline unknown & -4.263 & -4.396 & 0.2660 & 0.5110 & -4.174 & -4.330 & 0.0330 & 0.8154 & -4.347 & -4.463 & 0.3260 & 0.5288 \\
\hline unknown & -5.661 & -5.810 & 0.3730 & 0.5400 & -5.527 & -5.620 & 0.6110 & 0.8434 & -5.649 & -5.906 & 0.0750 & 0.5288 \\
\hline unknown & -3.315 & -3.339 & 0.6770 & 0.7797 & -3.209 & -3.104 & 0.6180 & 0.8492 & -3.037 & -3.309 & 0.2100 & 0.5288 \\
\hline unknown & -2.693 & -2.788 & 0.1100 & 0.5110 & -2.596 & -2.674 & 0.4400 & 0.8154 & -2.780 & -2.835 & 0.4920 & 0.5887 \\
\hline unknown & -5.687 & -5.725 & 0.6250 & 0.7360 & -5.552 & -5.583 & 0.8180 & 0.9335 & -5.719 & -5.757 & 0.7580 & 0.8153 \\
\hline unknown & -1.636 & -1.691 & 0.5780 & 0.6995 & -1.532 & -1.641 & 0.2540 & 0.8154 & -1.679 & -1.805 & 0.1320 & 0.5288 \\
\hline unknown & -4.735 & -4.880 & 0.0300 & 0.5110 & -4.653 & -4.863 & 0.1140 & 0.8154 & -4.978 & -4.835 & 0.2000 & 0.5288 \\
\hline unknown & -5.803 & -5.877 & 0.5080 & 0.6425 & -5.819 & -5.893 & 0.7190 & 0.8935 & -6.020 & -5.981 & 0.7070 & 0.7692 \\
\hline unknown & -6.250 & -6.588 & 0.3570 & 0.5297 & -6.424 & -6.312 & 0.5310 & 0.8240 & -6.602 & -6.091 & 0.2230 & 0.5288 \\
\hline unknown & -6.055 & -6.236 & 0.5280 & 0.6618 & -5.990 & -5.930 & 0.7700 & 0.9234 & -6.177 & -5.812 & 0.2360 & 0.5288 \\
\hline unknown & -1.816 & -1.798 & 0.8270 & 0.8741 & -1.674 & -1.626 & 0.7100 & 0.8894 & -1.561 & -1.865 & 0.1860 & 0.5288 \\
\hline unknown & -3.220 & -3.270 & 0.4320 & 0.5795 & -3.099 & -3.056 & 0.7900 & 0.9305 & -3.015 & -3.250 & 0.2360 & 0.5288 \\
\hline unknown & -4.867 & -5.067 & 0.1830 & 0.5110 & -4.812 & -5.000 & 0.2380 & 0.8154 & -5.137 & -5.306 & 0.1990 & 0.5288 \\
\hline unknown & -2.313 & -2.345 & 0.7230 & 0.8053 & -2.215 & -2.169 & 0.6720 & 0.8614 & -2.218 & -2.501 & 0.0770 & 0.5288 \\
\hline unknown & -5.168 & -5.195 & 0.7880 & 0.8522 & -5.127 & -4.985 & 0.4610 & 0.8202 & -4.971 & -5.228 & 0.2070 & 0.5288 \\
\hline unknown & -5.117 & -5.706 & 0.3930 & 0.5537 & -5.295 & -4.952 & 0.3310 & 0.8154 & -6.019 & -4.829 & 0.1930 & 0.5288 \\
\hline unknown & -4.000 & -4.081 & 0.2000 & 0.5110 & -3.858 & -3.991 & 0.1660 & 0.8154 & -4.045 & -4.052 & 0.8940 & 0.9179 \\
\hline unknown & -4.509 & -4.943 & 0.3580 & 0.5297 & -4.452 & -4.356 & 0.7290 & 0.8938 & -5.050 & -4.266 & 0.1610 & 0.5288 \\
\hline unknown & 2.187 & 2.110 & 0.2230 & 0.5110 & 2.195 & 2.251 & 0.4990 & 0.8215 & 2.138 & 2.088 & 0.3830 & 0.5291 \\
\hline unknown & -5.042 & -5.568 & 0.4050 & 0.5598 & -5.043 & -4.755 & 0.3380 & 0.8154 & -5.723 & -4.658 & 0.1650 & 0.5288 \\
\hline unknown & -5.775 & -5.744 & 0.7250 & 0.8053 & -5.737 & -5.972 & 0.2160 & 0.8154 & -6.152 & -5.818 & 0.0300 & 0.5288 \\
\hline unknown & -3.575 & -3.581 & 0.9450 & 0.9663 & -3.398 & -3.421 & 0.8360 & 0.9427 & -3.417 & -3.732 & 0.0650 & 0.5288 \\
\hline unknown & -2.767 & -2.846 & 0.4440 & 0.5868 & -2.679 & -2.810 & 0.1590 & 0.8154 & -2.784 & -3.008 & 0.0250 & 0.5288 \\
\hline unknown & -5.840 & -6.108 & 0.1720 & 0.5110 & -5.753 & -5.820 & 0.7150 & 0.8917 & -6.119 & -5.697 & 0.2250 & 0.5288 \\
\hline unknown & -5.911 & -5.959 & 0.5420 & 0.6715 & -5.784 & -5.769 & 0.8930 & 0.9595 & -5.988 & -5.953 & 0.6780 & 0.7444 \\
\hline unknown & -5.765 & -6.138 & 0.4540 & 0.5920 & -5.502 & -5.599 & 0.7950 & 0.9305 & -6.323 & -5.362 & 0.1420 & 0.5288 \\
\hline unknown & -3.845 & -4.626 & 0.3490 & 0.5293 & -3.510 & -3.959 & 0.5180 & 0.8229 & -5.125 & -3.747 & 0.2250 & 0.5288 \\
\hline unknown & -4.629 & -4.626 & 0.9600 & 0.9719 & -4.565 & -4.558 & 0.9410 & 0.9678 & -4.667 & -4.720 & 0.5480 & 0.6334 \\
\hline unknown & -6.849 & -7.758 & 0.2180 & 0.5110 & -6.589 & -7.244 & 0.3980 & 0.8154 & -7.796 & -7.097 & 0.4240 & 0.5434 \\
\hline unknown & -2.466 & -3.029 & 0.3280 & 0.5240 & -2.406 & -2.440 & 0.9440 & 0.9688 & -3.299 & -2.168 & 0.1330 & 0.5288 \\
\hline unknown & 0.193 & 0.211 & 0.8570 & 0.8943 & 0.250 & 0.192 & 0.6350 & 0.8537 & 0.261 & 0.050 & 0.0240 & 0.5288 \\
\hline unknown & -5.209 & -5.288 & 0.6340 & 0.7408 & -5.008 & -5.069 & 0.3710 & 0.8154 & -5.205 & -5.301 & 0.5120 & 0.6004 \\
\hline unknown & -3.947 & -4.037 & 0.2640 & 0.5110 & -3.952 & -3.978 & 0.8200 & 0.9335 & -4.105 & -4.130 & 0.7250 & 0.7833 \\
\hline unknown & -3.402 & -3.516 & 0.2610 & 0.5110 & -3.306 & -3.445 & 0.2720 & 0.8154 & -3.450 & -3.544 & 0.4030 & 0.5307 \\
\hline unknown & -3.145 & -3.239 & 0.2530 & 0.5110 & -3.083 & -3.122 & 0.6990 & 0.8844 & -3.172 & -3.277 & 0.0790 & 0.5288 \\
\hline unknown & -5.707 & -6.336 & 0.3460 & 0.5293 & -5.431 & -5.757 & 0.5140 & 0.8221 & -6.156 & -5.666 & 0.5130 & 0.6004 \\
\hline unknown & -5.112 & -5.266 & 0.0800 & 0.5110 & -5.036 & -5.371 & 0.0870 & 0.8154 & -5.420 & -5.183 & 0.1130 & 0.5288 \\
\hline unknown & -4.566 & -5.201 & 0.2420 & 0.5110 & -4.379 & -4.659 & 0.5880 & 0.8339 & -5.600 & -4.437 & 0.1760 & 0.5288 \\
\hline unknown & -5.649 & -6.147 & 0.3440 & 0.5293 & -5.253 & -5.646 & 0.4520 & 0.8202 & -6.458 & -5.459 & 0.2110 & 0.5288 \\
\hline unknown & -3.656 & -4.110 & 0.2150 & 0.5110 & -3.517 & -3.611 & 0.7600 & 0.9137 & -4.576 & -3.400 & 0.1040 & 0.5288 \\
\hline unknown & -3.240 & -3.305 & 0.5880 & 0.7066 & -3.214 & -3.139 & 0.4240 & 0.8154 & -3.199 & -3.444 & 0.0670 & 0.5288 \\
\hline unknown & -4.778 & -4.913 & 0.7250 & 0.8053 & -4.326 & -4.997 & 0.2600 & 0.8154 & -4.439 & -4.376 & 0.9210 & 0.9393 \\
\hline unknown & -0.090 & -0.899 & 0.2580 & 0.5110 & 0.076 & -0.129 & 0.7050 & 0.8844 & -1.497 & 0.050 & 0.1890 & 0.5288 \\
\hline unknown & -4.586 & -4.615 & 0.8250 & 0.8735 & -4.524 & -4.592 & 0.3290 & 0.8154 & -4.684 & -4.664 & 0.8120 & 0.8558 \\
\hline unknown & -0.559 & -1.256 & 0.2460 & 0.5110 & -0.460 & -0.513 & 0.9130 & 0.9647 & -1.508 & -0.263 & 0.1220 & 0.5288 \\
\hline unknown & -3.825 & -3.857 & 0.6820 & 0.7817 & -3.696 & -3.798 & 0.3250 & 0.8154 & -3.946 & -3.831 & 0.0710 & 0.5288 \\
\hline unknown & -5.521 & -5.974 & 0.3020 & 0.5110 & -5.662 & -5.290 & 0.0700 & 0.8154 & -6.011 & -5.388 & 0.2320 & 0.5288 \\
\hline unknown & -6.134 & -6.878 & 0.2620 & 0.5110 & -5.850 & -6.286 & 0.4830 & 0.8215 & -7.550 & -6.299 & 0.3140 & 0.5288 \\
\hline unknown & -5.505 & -6.341 & 0.1670 & 0.5110 & -5.240 & -5.372 & 0.7260 & 0.8936 & -6.801 & -5.445 & 0.2570 & 0.5288 \\
\hline unknown & -5.311 & -6.075 & 0.2040 & 0.5110 & -4.876 & -5.390 & 0.4230 & 0.8154 & -6.184 & -5.403 & 0.3830 & 0.5291 \\
\hline unknown & -4.236 & -4.414 & 0.5360 & 0.6683 & -4.186 & -4.079 & 0.6620 & 0.8614 & -4.532 & -3.948 & 0.1390 & 0.5288 \\
\hline unknown & -2.911 & -3.226 & 0.3130 & 0.5110 & -2.700 & -2.893 & 0.4940 & 0.8215 & -3.790 & -2.734 & 0.1390 & 0.5288 \\
\hline unknown & -2.693 & -2.923 & 0.3970 & 0.5567 & -2.569 & -2.534 & 0.8860 & 0.9543 & -3.537 & -2.533 & 0.1150 & 0.5288 \\
\hline unknown & -3.817 & -4.083 & 0.3600 & 0.5297 & -3.778 & -3.596 & 0.6050 & 0.8391 & -4.465 & -3.577 & 0.0570 & 0.5288 \\
\hline unknown & -0.832 & -1.751 & 0.2720 & 0.5110 & -0.244 & -1.030 & 0.2930 & 0.8154 & -2.252 & -0.870 & 0.2950 & 0.5288 \\
\hline unknown & -0.482 & -1.207 & 0.2540 & 0.5110 & -0.315 & -0.456 & 0.8130 & 0.9332 & -1.443 & -0.114 & 0.1280 & 0.5288 \\
\hline unknown & -4.454 & -4.961 & 0.3930 & 0.5537 & -4.090 & -4.477 & 0.3350 & 0.8154 & -5.096 & -4.274 & 0.2070 & 0.5288 \\
\hline unknown & -6.036 & -6.759 & 0.2110 & 0.5110 & -5.793 & -6.241 & 0.2840 & 0.8154 & -6.684 & -6.229 & 0.5570 & 0.6405 \\
\hline unknown & -4.356 & -4.675 & 0.3650 & 0.5329 & -4.352 & -4.175 & 0.5200 & 0.8229 & -4.996 & -4.187 & 0.1520 & 0.5288 \\
\hline unknown & -4.633 & -5.419 & 0.2110 & 0.5110 & -4.272 & -4.789 & 0.3070 & 0.8154 & -5.788 & -4.739 & 0.3360 & 0.5288 \\
\hline unknown & -2.092 & -2.158 & 0.3450 & 0.5293 & -1.991 & -2.015 & 0.7930 & 0.9305 & -2.075 & -2.152 & 0.3840 & 0.5291 \\
\hline unknown & -4.067 & -4.534 & 0.3760 & 0.5405 & -3.717 & -3.912 & 0.6340 & 0.8537 & -5.654 & -3.910 & 0.2310 & 0.5288 \\
\hline unknown & -5.143 & -5.155 & 0.8470 & 0.8862 & -5.021 & -5.048 & 0.8050 & 0.9324 & -5.061 & -5.138 & 0.4510 & 0.5611 \\
\hline unknown & -2.896 & -3.786 & 0.2590 & 0.5110 & -2.400 & -3.005 & 0.3300 & 0.8154 & -3.848 & -2.883 & 0.3830 & 0.5291 \\
\hline unknown & -0.512 & -1.155 & 0.2630 & 0.5110 & -0.318 & -0.267 & 0.8680 & 0.9543 & -1.452 & -0.326 & 0.2640 & 0.5288 \\
\hline unknown & -6.056 & -6.723 & 0.2300 & 0.5110 & -5.633 & -6.066 & 0.3290 & 0.8154 & -7.258 & -5.953 & 0.2240 & 0.5288 \\
\hline unknown & -1.361 & -1.968 & 0.3150 & 0.5110 & -1.085 & -1.058 & 0.9310 & 0.9664 & -2.191 & -1.188 & 0.3010 & 0.5288 \\
\hline unknown & -3.040 & -3.730 & 0.2550 & 0.5110 & -2.897 & -2.649 & 0.1560 & 0.8154 & -4.043 & -2.949 & 0.2610 & 0.5288 \\
\hline un & -2.460 & -3.089 & 0.2430 & 0.5110 & -2.238 & -2.321 & 0.8400 & 0.9427 & -3.164 & -2.262 & 0.2930 & 0. \\
\hline
\end{tabular}




\begin{tabular}{|c|c|c|c|c|c|c|c|c|c|c|c|c|}
\hline unknown & -3.320 & -3.787 & 0.3310 & 0.5269 & -2.886 & -3.266 & 0.2770 & 0.8154 & -4.326 & -3.176 & 0.1900 & 0.5288 \\
\hline unknown & -5.866 & -6.577 & 0.2300 & 0.5110 & -5.814 & -6.238 & 0.4700 & 0.8210 & -6.711 & -5.711 & 0.2270 & 0.5288 \\
\hline unknown & -2.055 & -2.094 & 0.7530 & 0.8234 & -1.804 & -1.963 & 0.2490 & 0.8154 & -2.786 & -1.943 & 0.1220 & 0.5288 \\
\hline unknown & -2.133 & -2.247 & 0.4100 & 0.5663 & -1.907 & -1.974 & 0.6650 & 0.8614 & -2.737 & -2.034 & 0.1190 & 0.5288 \\
\hline unknown & -5.491 & -5.517 & 0.7420 & 0.8137 & -5.340 & -5.448 & 0.4280 & 0.8154 & -5.525 & -5.550 & 0.8510 & 0.8888 \\
\hline unknown & -5.608 & -6.344 & 0.2030 & 0.5110 & -5.430 & -5.487 & 0.9090 & 0.9647 & -6.385 & -5.380 & 0.1690 & 0.5288 \\
\hline unknown & 1.246 & 0.401 & 0.2380 & 0.5110 & 1.757 & 1.108 & 0.2260 & 0.8154 & -0.056 & 1.215 & 0.2900 & 0.5288 \\
\hline unknown & -1.965 & -1.858 & 0.4230 & 0.5705 & -1.923 & -2.007 & 0.5090 & 0.8215 & -1.997 & -2.047 & 0.7930 & 0.8433 \\
\hline unknown & 2.687 & 2.113 & 0.2830 & 0.5110 & 2.986 & 2.771 & 0.4570 & 0.8202 & 1.700 & 2.828 & 0.2430 & 0.5288 \\
\hline unknown & 2.727 & 2.035 & 0.2100 & 0.5110 & 2.956 & 2.856 & 0.7540 & 0.9108 & 1.666 & 2.907 & 0.1970 & 0.5288 \\
\hline unknown & 2.012 & 1.233 & 0.1770 & 0.5110 & 2.159 & 2.107 & 0.8700 & 0.9543 & 0.931 & 2.129 & 0.2030 & 0.5288 \\
\hline unknown & -2.600 & -2.679 & 0.4010 & 0.5584 & -2.519 & -2.524 & 0.9540 & 0.9744 & -2.652 & -2.652 & 0.9970 & 0.9966 \\
\hline unknown & -0.326 & -1.062 & 0.1920 & 0.5110 & -0.139 & -0.325 & 0.7120 & 0.8896 & -1.248 & -0.035 & 0.1770 & 0.5288 \\
\hline unknown & -4.123 & -4.680 & 0.3270 & 0.5226 & -4.023 & -4.107 & 0.8110 & 0.9332 & -5.101 & -3.897 & 0.1960 & 0.5288 \\
\hline unknown & -3.697 & -3.729 & 0.6710 & 0.7756 & -3.602 & -3.627 & 0.7930 & 0.9305 & -3.694 & -3.689 & 0.8920 & 0.9179 \\
\hline unknown & -5.005 & -5.565 & 0.3730 & 0.5400 & -4.417 & -5.005 & 0.2210 & 0.8154 & -5.874 & -4.899 & 0.2990 & 0.5288 \\
\hline unknown & -5.503 & -6.001 & 0.3860 & 0.5509 & -5.392 & -5.361 & 0.9390 & 0.9675 & -6.434 & -5.203 & 0.2280 & 0.5288 \\
\hline unknown & -3.269 & -3.582 & 0.4200 & 0.5705 & -2.958 & -3.203 & 0.4000 & 0.8154 & -4.185 & -3.108 & 0.1600 & 0.5288 \\
\hline unknown & -5.098 & -5.547 & 0.3340 & 0.5286 & -4.675 & -5.052 & 0.2360 & 0.8154 & -5.853 & -4.834 & 0.2300 & 0.5288 \\
\hline unknown & -3.846 & -4.090 & 0.4150 & 0.5667 & -3.783 & -3.586 & 0.5550 & 0.8240 & -4.584 & -3.635 & 0.1310 & 0.5288 \\
\hline unknown & -3.710 & -4.307 & 0.3430 & 0.5293 & -3.205 & -3.767 & 0.1340 & 0.8154 & -4.900 & -3.623 & 0.2420 & 0.5288 \\
\hline unknown & -3.025 & -3.589 & 0.2240 & 0.5110 & -2.799 & -3.015 & 0.1780 & 0.8154 & -4.044 & -2.926 & 0.2020 & 0.5288 \\
\hline unknown & -1.159 & -2.006 & 0.2890 & 0.5110 & -0.576 & -1.212 & 0.2500 & 0.8154 & -2.348 & -1.097 & 0.3200 & 0.5288 \\
\hline unknown & -5.817 & -6.696 & 0.1450 & 0.5110 & -5.485 & -5.767 & 0.5550 & 0.8240 & -6.926 & -5.637 & 0.2180 & 0.5288 \\
\hline unknown & -6.020 & -6.082 & 0.2580 & 0.5110 & -5.925 & -5.935 & 0.9240 & 0.9664 & -6.032 & -6.206 & 0.0430 & 0.5288 \\
\hline unknown & -4.213 & -5.031 & 0.2370 & 0.5110 & -3.912 & -4.144 & 0.4450 & 0.8154 & -5.657 & -4.164 & 0.2860 & 0.5288 \\
\hline unknown & -0.245 & -1.099 & 0.2370 & 0.5110 & 0.068 & -0.046 & 0.7910 & 0.9305 & -1.343 & -0.122 & 0.2950 & 0.5288 \\
\hline unknown & -0.314 & -1.234 & 0.1930 & 0.5110 & -0.128 & -0.222 & 0.6480 & 0.8614 & -1.365 & -0.332 & 0.3660 & 0.5291 \\
\hline unknown & -4.633 & -5.183 & 0.2180 & 0.5110 & -4.357 & -4.737 & 0.3330 & 0.8154 & -5.829 & -4.736 & 0.2190 & 0.5288 \\
\hline unknown & -1.106 & -1.943 & 0.2700 & 0.5110 & -0.651 & -0.948 & 0.5410 & 0.8240 & -2.068 & -0.952 & 0.3320 & 0.5288 \\
\hline unknown & -2.346 & -2.604 & 0.3610 & 0.5297 & -2.075 & -2.257 & 0.2380 & 0.8154 & -3.262 & -2.238 & 0.1630 & 0.5288 \\
\hline unknown & -4.832 & -4.800 & 0.2910 & 0.5110 & -4.676 & -4.775 & 0.4430 & 0.8154 & -4.952 & -4.855 & 0.2280 & 0.5288 \\
\hline unknown & -2.092 & -1.957 & 0.1040 & 0.5110 & -1.934 & -1.966 & 0.7980 & 0.9305 & -2.468 & -2.026 & 0.1620 & 0.5288 \\
\hline unknown & -6.332 & -6.799 & 0.4520 & 0.5920 & -6.487 & -6.568 & 0.8490 & 0.9468 & -7.848 & -6.393 & 0.0610 & 0.5288 \\
\hline unknown & -2.710 & -3.481 & 0.1640 & 0.5110 & -2.371 & -3.038 & 0.2320 & 0.8154 & -3.512 & -2.890 & 0.4740 & 0.5720 \\
\hline unknown & -1.994 & -2.039 & 0.7160 & 0.8053 & -1.821 & -1.941 & 0.3850 & 0.8154 & -2.488 & -1.979 & 0.1210 & 0.5288 \\
\hline unknown & -5.839 & -6.392 & 0.1740 & 0.5110 & -5.585 & -5.633 & 0.8380 & 0.9427 & -6.464 & -5.691 & 0.1320 & 0.5288 \\
\hline unknown & -6.558 & -6.701 & 0.8060 & 0.8651 & -6.828 & -6.377 & 0.2970 & 0.8154 & -7.809 & -6.336 & 0.0690 & 0.5288 \\
\hline unknown & -3.171 & -3.633 & 0.1850 & 0.5110 & -2.969 & -3.144 & 0.6620 & 0.8614 & -3.892 & -3.060 & 0.1580 & 0.5288 \\
\hline unknown & -1.912 & -3.091 & 0.2260 & 0.5110 & -1.413 & -1.734 & 0.4080 & 0.8154 & -2.727 & -1.458 & 0.3450 & 0.5291 \\
\hline unknown & 3.371 & 2.846 & 0.2560 & 0.5110 & 3.619 & 3.380 & 0.2850 & 0.8154 & 2.377 & 3.480 & 0.2160 & 0.5288 \\
\hline unknown & 2.462 & 1.685 & 0.1950 & 0.5110 & 2.739 & 2.475 & 0.5570 & 0.8240 & 1.377 & 2.585 & 0.2520 & 0.5288 \\
\hline unknown & -1.320 & -2.207 & 0.1280 & 0.5110 & -1.093 & -1.553 & 0.4690 & 0.8210 & -2.188 & -1.117 & 0.2890 & 0.5288 \\
\hline unknown & -3.941 & -4.659 & 0.2630 & 0.5110 & -3.824 & -4.098 & 0.5970 & 0.8391 & -4.898 & -3.713 & 0.2830 & 0.5288 \\
\hline unknown & -4.023 & -4.546 & 0.2950 & 0.5110 & -3.591 & -4.120 & 0.1050 & 0.8154 & -5.035 & -4.063 & 0.2700 & 0.5288 \\
\hline unknown & -4.918 & -5.529 & 0.3490 & 0.5293 & -4.666 & -4.920 & 0.5770 & 0.8317 & -5.797 & -4.584 & 0.2690 & 0.5288 \\
\hline unknown & -2.509 & -2.830 & 0.2280 & 0.5110 & -2.292 & -2.507 & 0.2630 & 0.8154 & -3.414 & -2.464 & 0.1720 & 0.5288 \\
\hline unknown & -5.223 & -6.035 & 0.2160 & 0.5110 & -4.665 & -5.455 & 0.0660 & 0.8154 & -6.002 & -5.388 & 0.4560 & 0.5647 \\
\hline unknown & -3.451 & -3.921 & 0.2520 & 0.5110 & -3.258 & -3.314 & 0.8300 & 0.9427 & -4.322 & -3.389 & 0.2350 & 0.5288 \\
\hline unknown & -3.740 & -4.452 & 0.2250 & 0.5110 & -3.258 & -3.888 & 0.0940 & 0.8154 & -4.642 & -3.734 & 0.3320 & 0.5288 \\
\hline unknown & -1.993 & -1.981 & 0.8660 & 0.9010 & -1.884 & -2.010 & 0.3140 & 0.8154 & -2.077 & -1.962 & 0.2720 & 0.5288 \\
\hline unknown & -0.028 & -0.912 & 0.2210 & 0.5110 & 0.671 & -0.131 & 0.1290 & 0.8154 & -1.101 & 0.023 & 0.3620 & 0.5291 \\
\hline unknown & -7.119 & -7.464 & 0.6220 & 0.7352 & -6.855 & -6.777 & 0.8730 & 0.9543 & -8.326 & -6.680 & 0.1320 & 0.5288 \\
\hline unknown & -5.123 & -5.158 & 0.7200 & 0.8053 & -4.906 & -5.168 & 0.1580 & 0.8154 & -5.313 & -5.169 & 0.1880 & 0.5288 \\
\hline unknown & -5.317 & -5.690 & 0.0890 & 0.5110 & -5.057 & -5.595 & 0.1020 & 0.8154 & -5.732 & -5.446 & 0.4990 & 0.5937 \\
\hline unknown & -0.367 & -1.159 & 0.2180 & 0.5110 & 0.071 & -0.513 & 0.1490 & 0.8154 & -1.633 & -0.374 & 0.2850 & 0.5288 \\
\hline unknown & -2.013 & -2.938 & 0.1750 & 0.5110 & -1.443 & -2.167 & 0.1230 & 0.8154 & -3.056 & -2.158 & 0.4460 & 0.5587 \\
\hline unknown & -1.559 & -2.563 & 0.1670 & 0.5110 & -1.244 & -1.563 & 0.5220 & 0.8229 & -2.519 & -1.461 & 0.3670 & 0.5291 \\
\hline unknown & -1.498 & -2.517 & 0.1700 & 0.5110 & -1.197 & -1.567 & 0.3990 & 0.8154 & -2.548 & -1.449 & 0.3700 & 0.5291 \\
\hline unknown & -3.614 & -4.108 & 0.1800 & 0.5110 & -3.377 & -3.853 & 0.1900 & 0.8154 & -4.431 & -3.749 & 0.2530 & 0.5288 \\
\hline unknown & -3.667 & -3.835 & 0.2160 & 0.5110 & -3.438 & -3.704 & 0.1290 & 0.8154 & -3.800 & -3.529 & 0.4600 & 0.5658 \\
\hline unknown & -4.629 & -5.213 & 0.1240 & 0.5110 & -4.433 & -4.798 & 0.3100 & 0.8154 & -5.024 & -4.542 & 0.4860 & 0.5845 \\
\hline unknown & -1.907 & -1.999 & 0.6200 & 0.7337 & -1.749 & -1.840 & 0.2930 & 0.8154 & -2.622 & -1.779 & 0.0920 & 0.5288 \\
\hline unknown & -6.139 & -6.774 & 0.3100 & 0.5110 & -6.125 & -6.058 & 0.8840 & 0.9543 & -7.091 & -6.156 & 0.2520 & 0.5288 \\
\hline unknown & -3.457 & -3.994 & 0.2170 & 0.5110 & -3.097 & -3.384 & 0.1610 & 0.8154 & -4.510 & -3.315 & 0.1950 & 0.5288 \\
\hline unknown & -1.817 & -1.670 & 0.3470 & 0.5293 & -1.724 & -1.799 & 0.6570 & 0.8614 & -2.077 & -1.806 & 0.2290 & 0.5288 \\
\hline unknown & -5.268 & -5.727 & 0.4550 & 0.5920 & -5.617 & -5.194 & 0.1650 & 0.8154 & -6.694 & -5.278 & 0.1020 & 0.5288 \\
\hline unknown & -4.707 & -5.512 & 0.1920 & 0.5110 & -4.455 & -4.884 & 0.4080 & 0.8154 & -5.431 & -4.894 & 0.4380 & 0.5518 \\
\hline unknown & -5.646 & -5.719 & 0.3130 & 0.5110 & -5.565 & -5.590 & 0.8350 & 0.9427 & -5.633 & -5.717 & 0.4700 & 0.5691 \\
\hline unknown & -4.017 & -3.796 & 0.3210 & 0.5151 & -3.978 & -3.988 & 0.9610 & 0.9768 & -4.251 & -4.028 & 0.4050 & 0.5307 \\
\hline unknown & -1.865 & -2.052 & 0.1880 & 0.5110 & -1.664 & -1.856 & 0.3060 & 0.8154 & -2.460 & -1.821 & 0.1470 & 0.5288 \\
\hline unknown & -5.513 & -5.620 & 0.8460 & 0.8862 & -5.660 & -5.609 & 0.9260 & 0.9664 & -6.992 & -5.203 & 0.0570 & 0.5288 \\
\hline unknown & -5.584 & -5.767 & 0.0550 & 0.5110 & -5.581 & -5.560 & 0.8540 & 0.9474 & -5.652 & -5.682 & 0.8110 & 0.8558 \\
\hline unknown & -3.703 & -4.394 & 0.1060 & 0.5110 & -3.569 & -3.883 & 0.5100 & 0.8215 & -4.590 & -3.582 & 0.1790 & 0.5288 \\
\hline unknown & 3.006 & 2.264 & 0.2140 & 0.5110 & 3.458 & 2.893 & 0.1010 & 0.8154 & 1.958 & 3.026 & 0.3180 & 0.5288 \\
\hline unknown & 2.954 & 2.218 & 0.1620 & 0.5110 & 3.230 & 2.883 & 0.2140 & 0.8154 & 1.895 & 3.085 & 0.2620 & 0.5288 \\
\hline $\mathrm{vn}$ & -4.652 & -5.278 & 0.1190 & 0.5110 & -4.328 & -4.970 & 0.1680 & 0.8154 & -5.374 & -4.190 & 0.3200 & . \\
\hline
\end{tabular}




\begin{tabular}{|c|c|c|c|c|c|c|c|c|c|c|c|c|}
\hline unknown & 0.710 & -0.296 & 0.1460 & 0.5110 & 1.030 & 0.673 & 0.5490 & 0.8240 & -0.191 & 0.844 & 0.3760 & 0.5291 \\
\hline unknown & -2.017 & -2.688 & 0.3080 & 0.5110 & -1.800 & -2.215 & 0.2710 & 0.8154 & -3.172 & -1.849 & 0.2510 & 0.5288 \\
\hline unknown & -4.254 & -4.765 & 0.1320 & 0.5110 & -4.068 & -4.276 & 0.0430 & 0.8154 & -4.357 & -4.201 & 0.7610 & 0.8164 \\
\hline unknown & -5.184 & -5.564 & 0.1480 & 0.5110 & -5.157 & -5.240 & 0.6700 & 0.8614 & -5.195 & -5.163 & 0.9220 & 0.9393 \\
\hline unknown & -3.491 & -4.163 & 0.0980 & 0.5110 & -3.173 & -3.660 & 0.3710 & 0.8154 & -3.728 & -3.204 & 0.4960 & 0.5923 \\
\hline unknown & -3.642 & -4.045 & 0.3170 & 0.5110 & -3.163 & -3.758 & 0.0700 & 0.8154 & -4.482 & -3.673 & 0.2750 & 0.5288 \\
\hline unknown & -3.765 & -4.134 & 0.2530 & 0.5110 & -3.396 & -3.850 & 0.0760 & 0.8154 & -4.618 & -3.810 & 0.2550 & 0.5288 \\
\hline unknown & -3.320 & -3.846 & 0.1870 & 0.5110 & -2.956 & -3.319 & 0.0660 & 0.8154 & -4.346 & -3.188 & 0.2040 & 0.5288 \\
\hline unknown & -6.929 & -7.023 & 0.8700 & 0.9016 & -6.981 & -6.893 & 0.8620 & 0.9524 & -8.632 & -6.798 & 0.0710 & 0.5288 \\
\hline unknown & -4.554 & -5.191 & 0.1870 & 0.5110 & -4.187 & -4.496 & 0.3850 & 0.8154 & -5.396 & -4.561 & 0.3130 & 0.5288 \\
\hline unknown & -4.142 & -4.800 & 0.2150 & 0.5110 & -3.694 & -4.105 & 0.3720 & 0.8154 & -4.964 & -3.969 & 0.2670 & 0.5288 \\
\hline unknown & -4.875 & -5.618 & 0.1400 & 0.5110 & -4.457 & -4.819 & 0.4600 & 0.8202 & -5.569 & -4.727 & 0.3250 & 0.5288 \\
\hline unknown & -5.764 & -5.763 & 0.9920 & 0.9951 & -5.449 & -5.670 & 0.2070 & 0.8154 & -5.814 & -5.665 & 0.5860 & 0.6677 \\
\hline unknown & -5.409 & -5.658 & 0.4550 & 0.5920 & -5.235 & -5.097 & 0.4550 & 0.8202 & -6.025 & -5.250 & 0.2280 & 0.5288 \\
\hline unknown & -5.925 & -6.166 & 0.4370 & 0.5821 & -5.787 & -5.485 & 0.2400 & 0.8154 & -6.389 & -5.731 & 0.2730 & 0.5288 \\
\hline unknown & -2.644 & -3.425 & 0.2490 & 0.5110 & -2.076 & -2.678 & 0.1690 & 0.8154 & -3.585 & -2.672 & 0.3890 & 0.5291 \\
\hline unknown & -5.168 & -5.248 & 0.4020 & 0.5584 & -5.305 & -5.067 & 0.2950 & 0.8154 & -5.368 & -5.465 & 0.8200 & 0.8613 \\
\hline unknown & -2.485 & -3.379 & 0.1950 & 0.5110 & -2.230 & -2.566 & 0.3400 & 0.8154 & -3.572 & -2.357 & 0.3110 & 0.5288 \\
\hline unknown & -5.938 & -6.799 & 0.2310 & 0.5110 & -5.497 & -6.250 & 0.0700 & 0.8154 & -6.736 & -6.053 & 0.4610 & 0.5658 \\
\hline unknown & -4.610 & -5.039 & 0.0640 & 0.5110 & -4.454 & -4.768 & 0.2030 & 0.8154 & -5.072 & -4.744 & 0.3750 & 0.5291 \\
\hline unknown & -4.933 & -5.605 & 0.2130 & 0.5110 & -4.689 & -4.834 & 0.6020 & 0.8391 & -5.613 & -4.913 & 0.3960 & 0.5291 \\
\hline unknown & -4.431 & -5.151 & 0.1560 & 0.5110 & -4.281 & -4.350 & 0.8410 & 0.9427 & -4.746 & -4.319 & 0.5820 & 0.6640 \\
\hline unknown & -4.997 & -5.890 & 0.1290 & 0.5110 & -4.944 & -5.027 & 0.7460 & 0.9074 & -5.269 & -5.066 & 0.8020 & 0.8494 \\
\hline unknown & -3.082 & -3.577 & 0.1860 & 0.5110 & -2.838 & -3.228 & 0.1580 & 0.8154 & -3.708 & -3.177 & 0.2690 & 0.5288 \\
\hline unknown & -5.747 & -6.453 & 0.2240 & 0.5110 & -5.339 & -5.580 & 0.4900 & 0.8215 & -5.961 & -5.557 & 0.6360 & 0.7143 \\
\hline unknown & -6.510 & -7.764 & 0.0610 & 0.5110 & -5.959 & -7.185 & 0.0950 & 0.8154 & -7.048 & -6.999 & 0.9450 & 0.9538 \\
\hline unknown & -5.943 & -6.030 & 0.5770 & 0.6995 & -5.810 & -5.934 & 0.2860 & 0.8154 & -5.972 & -5.849 & 0.4500 & 0.5609 \\
\hline unknown & -5.255 & -5.545 & 0.1220 & 0.5110 & -5.120 & -5.316 & 0.2260 & 0.8154 & -5.767 & -5.327 & 0.1980 & 0.5288 \\
\hline unknown & -1.716 & -1.794 & 0.4690 & 0.6010 & -1.592 & -1.686 & 0.5130 & 0.8221 & -2.313 & -1.689 & 0.1350 & 0.5288 \\
\hline unknown & -5.518 & -5.570 & 0.5650 & 0.6898 & -5.443 & -5.442 & 0.9910 & 0.9914 & -5.556 & -5.538 & 0.8150 & 0.8573 \\
\hline unknown & -1.624 & -1.719 & 0.3610 & 0.5297 & -1.487 & -1.636 & 0.2560 & 0.8154 & -2.226 & -1.630 & 0.1460 & 0.5288 \\
\hline unknown & -6.527 & -6.616 & 0.8020 & 0.8632 & -6.727 & -6.775 & 0.9130 & 0.9647 & -7.569 & -6.664 & 0.0950 & 0.5288 \\
\hline unknown & -4.864 & -5.282 & 0.2940 & 0.5110 & -4.651 & -5.015 & 0.2970 & 0.8154 & -5.818 & -4.764 & 0.2080 & 0.5288 \\
\hline unknown & -2.417 & -3.050 & 0.1060 & 0.5110 & -2.155 & -2.489 & 0.3110 & 0.8154 & -3.323 & -2.407 & 0.2320 & 0.5288 \\
\hline unknown & -5.766 & -6.345 & 0.0870 & 0.5110 & -5.487 & -5.883 & 0.2810 & 0.8154 & -6.139 & -5.591 & 0.3710 & 0.5291 \\
\hline unknown & -4.667 & -5.612 & 0.0580 & 0.5110 & -4.472 & -5.239 & 0.0690 & 0.8154 & -5.459 & -4.991 & 0.4290 & 0.5483 \\
\hline unknown & -5.389 & -5.835 & 0.2180 & 0.5110 & -5.015 & -5.483 & 0.3760 & 0.8154 & -5.737 & -5.126 & 0.3780 & 0.5291 \\
\hline unknown & -1.373 & -2.527 & 0.1930 & 0.5110 & -0.986 & -1.746 & 0.1380 & 0.8154 & -2.439 & -1.538 & 0.3970 & 0.5291 \\
\hline unknown & -6.280 & -6.703 & 0.3480 & 0.5293 & -6.016 & -6.397 & 0.2250 & 0.8154 & -6.890 & -6.351 & 0.5030 & 0.5957 \\
\hline unknown & -2.134 & -3.045 & 0.2100 & 0.5110 & -1.916 & -2.319 & 0.2470 & 0.8154 & -3.070 & -1.960 & 0.3040 & 0.5288 \\
\hline unknown & -2.997 & -3.705 & 0.3060 & 0.5110 & -2.501 & -3.235 & 0.0350 & 0.8154 & -3.968 & -3.169 & 0.4310 & 0.5493 \\
\hline unknown & -4.354 & -5.070 & 0.2870 & 0.5110 & -4.067 & -4.282 & 0.6320 & 0.8537 & -4.642 & -4.225 & 0.6480 & 0.7243 \\
\hline unknown & -3.676 & -4.532 & 0.1660 & 0.5110 & -3.485 & -4.069 & 0.2630 & 0.8154 & -4.540 & -3.637 & 0.3810 & 0.5291 \\
\hline unknown & -5.846 & -6.088 & 0.0860 & 0.5110 & -5.751 & -5.905 & 0.2790 & 0.8154 & -5.893 & -5.775 & 0.6070 & 0.6874 \\
\hline unknown & -6.392 & -6.849 & 0.1320 & 0.5110 & -5.980 & -6.626 & 0.0720 & 0.8154 & -7.776 & -6.477 & 0.2150 & 0.5288 \\
\hline unknown & -5.269 & -5.733 & 0.2760 & 0.5110 & -4.905 & -5.413 & 0.1180 & 0.8154 & -6.097 & -5.172 & 0.2600 & 0.5288 \\
\hline unknown & -5.896 & -6.382 & 0.1990 & 0.5110 & -5.734 & -6.588 & 0.1890 & 0.8154 & -6.249 & -5.750 & 0.3040 & 0.5288 \\
\hline unknown & -4.732 & -4.972 & 0.4150 & 0.5667 & -4.528 & -4.501 & 0.8700 & 0.9543 & -5.293 & -4.578 & 0.2530 & 0.5288 \\
\hline unknown & -5.164 & -5.321 & 0.5750 & 0.6995 & -4.947 & -4.913 & 0.8540 & 0.9474 & -5.699 & -4.904 & 0.2310 & 0.5288 \\
\hline unknown & -5.730 & -6.226 & 0.3920 & 0.5537 & -5.291 & -5.793 & 0.1730 & 0.8154 & -6.274 & -5.669 & 0.4670 & 0.5671 \\
\hline unknown & -4.579 & -4.965 & 0.1820 & 0.5110 & -4.412 & -4.515 & 0.6250 & 0.8537 & -5.340 & -4.533 & 0.2540 & 0.5288 \\
\hline unknown & -5.204 & -5.760 & 0.3430 & 0.5293 & -4.842 & -5.101 & 0.3760 & 0.8154 & -5.598 & -5.045 & 0.5040 & 0.5957 \\
\hline unknown & -6.203 & -6.811 & 0.0570 & 0.5110 & -6.101 & -6.341 & 0.2140 & 0.8154 & -6.818 & -6.389 & 0.2930 & 0.5288 \\
\hline unknown & -5.935 & -6.923 & 0.1150 & 0.5110 & -5.784 & -5.741 & 0.9000 & 0.9601 & -6.235 & -5.771 & 0.5740 & 0.6580 \\
\hline unknown & -6.624 & -7.420 & 0.2300 & 0.5110 & -6.241 & -6.455 & 0.5730 & 0.8315 & -7.238 & -6.471 & 0.4010 & 0.5307 \\
\hline unknown & -6.025 & -6.519 & 0.1920 & 0.5110 & -5.957 & -5.969 & 0.9130 & 0.9647 & -6.077 & -5.976 & 0.8340 & 0.8743 \\
\hline unknown & -5.739 & -5.834 & 0.3940 & 0.5541 & -5.549 & -5.545 & 0.9800 & 0.9857 & -6.253 & -5.664 & 0.2070 & 0.5288 \\
\hline unknown & -6.212 & -6.768 & 0.2180 & 0.5110 & -5.895 & -5.999 & 0.6360 & 0.8537 & -6.890 & -6.026 & 0.2360 & 0.5288 \\
\hline unknown & -4.341 & -5.121 & 0.1940 & 0.5110 & -3.933 & -4.620 & 0.1150 & 0.8154 & -5.329 & -4.625 & 0.3360 & 0.5288 \\
\hline unknown & -6.281 & -6.708 & 0.2910 & 0.5110 & -5.957 & -6.593 & 0.1380 & 0.8154 & -6.979 & -6.444 & 0.3100 & 0.5288 \\
\hline unknown & -5.093 & -5.917 & 0.1580 & 0.5110 & -4.952 & -5.309 & 0.3340 & 0.8154 & -5.938 & -5.058 & 0.3040 & 0.5288 \\
\hline unknown & -5.695 & -6.212 & 0.2870 & 0.5110 & -5.500 & -5.634 & 0.8170 & 0.9335 & -7.036 & -5.399 & 0.1840 & 0.5288 \\
\hline unknown & -5.660 & -6.116 & 0.1530 & 0.5110 & -5.401 & -5.841 & 0.0770 & 0.8154 & -6.280 & -5.602 & 0.1680 & 0.5288 \\
\hline unknown & -6.097 & -6.923 & 0.1280 & 0.5110 & -5.829 & -6.046 & 0.5660 & 0.8293 & -6.232 & -6.015 & 0.7640 & 0.8164 \\
\hline unknown & -5.523 & -6.407 & 0.1970 & 0.5110 & -5.524 & -5.764 & 0.4840 & 0.8215 & -6.075 & -5.825 & 0.6730 & 0.7426 \\
\hline unknown & -6.031 & -6.858 & 0.1760 & 0.5110 & -6.060 & -6.262 & 0.5000 & 0.8215 & -6.586 & -6.335 & 0.6770 & 0.7444 \\
\hline unknown & -5.106 & -5.807 & 0.1930 & 0.5110 & -5.104 & -5.224 & 0.6690 & 0.8614 & -5.451 & -5.137 & 0.6550 & 0.7292 \\
\hline unknown & -6.270 & -6.640 & 0.3540 & 0.5297 & -6.073 & -6.030 & 0.8420 & 0.9427 & -6.141 & -6.057 & 0.8920 & 0.9179 \\
\hline unknown & -5.535 & -6.433 & 0.1760 & 0.5110 & -5.331 & -5.844 & 0.3800 & 0.8154 & -6.234 & -5.404 & 0.3860 & 0.5291 \\
\hline unknown & -6.552 & -7.020 & 0.3420 & 0.5293 & -6.592 & -6.671 & 0.7560 & 0.9113 & -6.805 & -6.458 & 0.6080 & 0.6874 \\
\hline unknown & -6.249 & -6.726 & 0.1660 & 0.5110 & -5.976 & -6.292 & 0.1060 & 0.8154 & -6.834 & -6.187 & 0.2600 & 0.5288 \\
\hline unknown & -6.046 & -6.612 & 0.1690 & 0.5110 & -5.791 & -6.173 & 0.0210 & 0.8154 & -7.111 & -5.897 & 0.1590 & 0.5288 \\
\hline unknown & -6.110 & -6.234 & 0.1890 & 0.5110 & -6.033 & -6.156 & 0.4630 & 0.8202 & -6.154 & -6.240 & 0.4370 & 0.5518 \\
\hline unknown & -4.938 & -4.973 & 0.7340 & 0.8094 & -4.884 & -4.979 & 0.4850 & 0.8215 & -5.214 & -4.887 & 0.0420 & 0.5288 \\
\hline unknown & -4.971 & -4.926 & 0.5370 & 0.6684 & -4.862 & -4.999 & 0.3840 & 0.8154 & -5.131 & -4.978 & 0.1990 & 0.5288 \\
\hline unknown & -5.062 & -5.086 & 0.8140 & 0.8667 & -4.970 & -5.183 & 0.1900 & 0.8154 & -5.237 & -5.057 & 0.0560 & 0.5288 \\
\hline unknown & -5.813 & -5.806 & 0.9180 & 0.9461 & -5.641 & -5.492 & 0.6210 & 0.8505 & -5.291 & -5.915 & 0.0990 & 0.5288 \\
\hline
\end{tabular}




\begin{tabular}{lllllllllllll} 
unknown & -4.736 & -4.772 & 0.6640 & 0.7695 & -4.688 & -4.400 & 0.3650 & 0.8154 & -4.238 & -4.720 & 0.2150 & 0.5288 \\
unknown & -5.135 & -5.217 & 0.2640 & 0.5110 & -5.071 & -4.861 & 0.5300 & 0.8240 & -4.671 & -5.180 & 0.1830 & 0.5288 \\
unknown & -4.816 & -4.819 & 0.9500 & 0.9665 & -4.726 & -4.894 & 0.3200 & 0.8154 & -4.820 & -4.819 & 0.9890 & 0.9928 \\
unknown & -5.099 & -5.125 & 0.7200 & 0.8053 & -5.062 & -5.210 & 0.3560 & 0.8154 & -5.178 & -5.138 & 0.6660 & 0.7403 \\
unknown & -6.107 & -6.189 & 0.4860 & 0.6199 & -5.943 & -6.143 & 0.1460 & 0.8154 & -6.125 & -6.216 & 0.3280 & 0.5288 \\
unknown & -4.956 & -4.881 & 0.2680 & 0.5110 & -4.809 & -4.967 & 0.3800 & 0.8154 & -5.158 & -4.862 & 0.1840 & 0.5288 \\
unknown & -4.915 & -4.886 & 0.7090 & 0.8002 & -4.696 & -4.959 & 0.2270 & 0.8154 & -4.951 & -4.817 & 0.4910 & 0.5887 \\
unknown & -4.805 & -4.893 & 0.3560 & 0.5297 & -4.769 & -4.946 & 0.2510 & 0.8154 & -5.140 & -4.805 & 0.1600 & 0.5288 \\
unknown & -0.125 & -0.204 & 0.3560 & 0.5297 & -0.102 & -0.142 & 0.7720 & 0.9234 & -0.253 & -0.242 & 0.8590 & 0.8955 \\
unknown & -3.625 & -3.754 & 0.5080 & 0.6425 & -3.657 & -3.479 & 0.1320 & 0.8154 & -3.779 & -3.777 & 0.9950 & 0.9966 \\
\hline
\end{tabular}

${ }^{\mathrm{a}}$ The ambiguous subspecies indicates that the lipidomic profiling gave two possible identifications of the lipid. ${ }^{\mathrm{b}}$ Abbreviations: ChoE, cholesteryl ester. SM, sphingomyelin. TG, triacylglycerol. LysoPC, lysophophosphatidylcholine. PC, phosphatidylcholine. 
Online Resource Table S6

Results from the analysis of variance between the control group $(\mathrm{CON})$ and the high-energy feeding group (HIGH) within time points in adipose tissue negative electrospray ionization mode (ESI-) dataset. $P$-values were obtained from the analysis performed using MIXED procedure in SAS, with diet as the fixed effect and pair as the random effect. Adjusted- $p$ values were obtained from $p$-values after false discovery rate control.

\begin{tabular}{|c|c|c|c|c|c|c|c|c|c|c|c|c|}
\hline \multirow[b]{2}{*}{ Lipid subspecies } & \multicolumn{4}{|c|}{$-8 \mathrm{~d}$} & \multicolumn{4}{|c|}{$1 \mathrm{~d}$} & \multicolumn{4}{|c|}{$9 \mathrm{~d}$} \\
\hline & $\mathrm{CON}$ & HIGH & $p$-value & Adjusted- $p$ & $\mathrm{CON}$ & HIGH & $p$-value & Adjusted- $p$ & $\mathrm{CON}$ & HIGH & $p$-value & Adjusted- $p$ \\
\hline$\overline{\operatorname{Cer}(\mathrm{d} 17: 1 / 22: 0)}$ & -9.877 & -8.390 & $<.0001$ & 0.0017 & -9.642 & -8.984 & 0.1735 & 0.9350 & -9.832 & -9.636 & 0.6296 & 0.8091 \\
\hline Cer(d18:1/16:0) & -6.841 & -6.438 & 0.2983 & 0.6093 & -6.227 & -6.017 & 0.6875 & 0.9368 & -6.773 & -6.635 & 0.8288 & 0.9103 \\
\hline Cer(d18:1/18:0) & -8.570 & -8.354 & 0.5329 & 0.7826 & -7.683 & -7.378 & 0.6042 & 0.9350 & -7.452 & -8.097 & 0.1976 & 0.4721 \\
\hline $\operatorname{Cer}(\mathrm{d} 18: 1 / 20: 0)$ & -10.999 & -9.443 & $<.0001$ & 0.0028 & -10.485 & -9.606 & 0.2083 & 0.9350 & -10.501 & -10.347 & 0.7451 & 0.8690 \\
\hline $\operatorname{Cer}(\mathrm{d} 18: 1 / 22: 0)$ & -6.850 & -5.737 & 0.0004 & 0.0553 & -6.617 & -6.162 & 0.3423 & 0.9350 & -7.137 & -6.879 & 0.5311 & 0.7413 \\
\hline $\operatorname{Cer}(\mathrm{d} 18: 1 / 22: 1)$ & -10.511 & -9.604 & 0.0049 & 0.1659 & -10.325 & -9.517 & 0.2555 & 0.9350 & -10.403 & -10.245 & 0.7648 & 0.8835 \\
\hline $\operatorname{Cer}(\mathrm{d} 18: 1 / 23: 0)$ & -6.585 & -5.617 & 0.0002 & 0.0473 & -6.417 & -6.175 & 0.4855 & 0.9350 & -6.755 & -6.681 & 0.8363 & 0.9153 \\
\hline $\operatorname{Cer}(\mathrm{d} 18: 1 / 23: 1)$ & -8.329 & -7.174 & 0.0016 & 0.1153 & -8.419 & -7.836 & 0.3125 & 0.9350 & -8.785 & -8.458 & 0.3905 & 0.6413 \\
\hline $\operatorname{Cer}(\mathrm{d} 18: 1 / 24: 0)$ & -6.772 & -6.345 & 0.0102 & 0.1949 & -6.391 & -6.303 & 0.8132 & 0.9665 & -6.485 & -6.726 & 0.5671 & 0.7685 \\
\hline Cer(d18:1/24:1) & -8.151 & -7.345 & 0.0056 & 0.1705 & -7.880 & -7.272 & 0.2751 & 0.9350 & -8.230 & -7.993 & 0.5305 & 0.7413 \\
\hline Cer(d18:1/25:0) & -9.906 & -9.705 & 0.2526 & 0.5708 & -9.417 & -9.816 & 0.2645 & 0.9350 & -9.852 & -9.900 & 0.8750 & 0.9333 \\
\hline $\operatorname{Cer}(\mathrm{d} 18: 1 / 25: 1)$ & -9.412 & -8.968 & 0.0118 & 0.2113 & -9.279 & -9.320 & 0.9331 & 0.9848 & -9.726 & -9.625 & 0.7191 & 0.8519 \\
\hline Cer(d18:2/16:0) & -11.780 & -11.319 & 0.1973 & 0.5141 & -11.110 & -10.270 & 0.3536 & 0.9350 & -11.343 & -11.072 & 0.7361 & 0.8636 \\
\hline Cer(d18:2/18:0) & -11.427 & -11.305 & 0.6648 & 0.8350 & -10.824 & -10.235 & 0.4970 & 0.9350 & -10.561 & -10.913 & 0.5006 & 0.7238 \\
\hline $\operatorname{Cer}(\mathrm{d} 18: 2 / 24: 1)$ & -11.832 & -11.393 & 0.2873 & 0.6073 & -11.289 & -10.621 & 0.3748 & 0.9350 & -11.560 & -11.487 & 0.9136 & 0.9466 \\
\hline HexCer(d18:1/22:0) & -13.722 & -12.683 & 0.3554 & 0.6476 & -11.298 & -11.532 & 0.8664 & 0.9665 & -11.319 & -12.214 & 0.3211 & 0.5780 \\
\hline PC(18:1/18:0) & -5.392 & -4.946 & 0.0418 & 0.3335 & -5.425 & -5.289 & 0.6980 & 0.9368 & -5.184 & -5.581 & 0.1988 & 0.4721 \\
\hline $\mathrm{PC}(36: 2)$ & -6.832 & -6.591 & 0.3588 & 0.6476 & -6.614 & -6.553 & 0.8282 & 0.9665 & -6.214 & -6.664 & 0.1686 & 0.4440 \\
\hline $\mathrm{PC}(36: 3)$ & -7.928 & -7.239 & 0.0036 & 0.1599 & -8.110 & -8.183 & 0.8414 & 0.9665 & -8.028 & -8.260 & 0.1105 & 0.4114 \\
\hline $\mathrm{PC}(38: 3)$ & -7.630 & -7.147 & 0.0367 & 0.3101 & -7.483 & -7.685 & 0.6794 & 0.9368 & -8.038 & -8.303 & 0.2427 & 0.5121 \\
\hline \multicolumn{13}{|l|}{$\operatorname{PE}(16: 0 / 20: 4)+$} \\
\hline $\operatorname{PE}(18: 2 / 18: 2)^{\mathrm{a}}$ & -9.802 & -9.047 & 0.0039 & 0.1599 & -9.908 & -9.728 & 0.6193 & 0.9350 & -9.676 & -9.769 & 0.6201 & 0.8041 \\
\hline $\operatorname{PE}(18: 0 / 16: 1)$ & -8.315 & -7.647 & 0.0298 & 0.2953 & -8.217 & -8.119 & 0.7979 & 0.9665 & -7.673 & -8.523 & 0.0881 & 0.3977 \\
\hline $\operatorname{PE}(18: 0 / 18: 1)$ & -6.634 & -6.162 & 0.0938 & 0.4262 & -6.485 & -6.417 & 0.8176 & 0.9665 & -5.805 & -6.746 & 0.1483 & 0.4361 \\
\hline PE(18:0/18:2) & -7.633 & -7.314 & 0.2361 & 0.5553 & -7.436 & -7.452 & 0.9440 & 0.9848 & -6.593 & -7.295 & 0.1074 & 0.4114 \\
\hline PE(18:0/20:0) & -9.004 & -8.531 & 0.0009 & 0.0907 & -9.006 & -8.850 & 0.6893 & 0.9368 & -8.575 & -9.425 & 0.0498 & 0.3934 \\
\hline $\operatorname{PE}(18: 0 / 20: 3)$ & -8.791 & -8.538 & 0.1116 & 0.4448 & -8.703 & -8.457 & 0.5988 & 0.9350 & -8.595 & -9.272 & 0.0766 & 0.3934 \\
\hline $\operatorname{PE}(18: 0 / 20: 4)$ & -8.816 & -8.036 & 0.0025 & 0.1459 & -8.749 & -8.782 & 0.9330 & 0.9848 & -8.605 & -8.980 & 0.2360 & 0.5053 \\
\hline $\mathrm{PE}(18: 0 / 22: 6)$ & -9.995 & -9.834 & 0.2268 & 0.5509 & -10.044 & -9.972 & 0.8079 & 0.9665 & -9.565 & -10.120 & 0.1375 & 0.4345 \\
\hline PE(18:1/18:0) & -7.543 & -7.338 & 0.4245 & 0.7043 & -7.314 & -7.019 & 0.4512 & 0.9350 & -6.703 & -7.590 & 0.0800 & 0.3938 \\
\hline $\operatorname{PE}(34: 1)$ & -7.977 & -7.621 & 0.1718 & 0.4907 & -7.695 & -7.755 & 0.8495 & 0.9665 & -7.241 & -7.857 & 0.0926 & 0.4048 \\
\hline $\operatorname{PE}(36: 2)$ & -8.571 & -8.222 & 0.2071 & 0.5259 & -8.206 & -8.329 & 0.5738 & 0.9350 & -7.472 & -7.984 & 0.0716 & 0.3934 \\
\hline $\operatorname{PE}(36: 2)$ & -8.022 & -7.692 & 0.4400 & 0.7141 & -7.707 & -7.608 & 0.8394 & 0.9665 & -7.172 & -8.036 & 0.0843 & 0.3940 \\
\hline $\operatorname{PE}(36: 2 \mathrm{e})$ & -8.646 & -7.527 & 0.0508 & 0.3484 & -8.162 & -8.354 & 0.6393 & 0.9368 & -7.596 & -8.425 & 0.2994 & 0.5694 \\
\hline $\operatorname{PE}(36: 3)$ & -9.887 & -9.808 & 0.8370 & 0.8868 & -9.437 & -9.422 & 0.9695 & 0.9876 & -8.926 & -9.704 & 0.1515 & 0.4361 \\
\hline $\operatorname{PE}(36: 3 e)$ & -9.337 & -8.518 & 0.2930 & 0.6073 & -8.547 & -9.040 & 0.4468 & 0.9350 & -8.023 & -9.533 & 0.1341 & 0.4278 \\
\hline $\operatorname{PE}(36: 5 e)$ & -9.622 & -9.167 & 0.1480 & 0.4668 & -9.660 & -9.886 & 0.5229 & 0.9350 & -9.319 & -9.866 & 0.2615 & 0.5276 \\
\hline $\mathrm{PE}(36: 5 \mathrm{e})$ & -6.370 & -5.983 & 0.1397 & 0.4558 & -6.159 & -6.104 & 0.8382 & 0.9665 & -5.661 & -6.194 & 0.1840 & 0.4554 \\
\hline $\operatorname{PE}(38: 4)$ & -11.184 & -10.759 & 0.2486 & 0.5663 & -10.987 & -10.798 & 0.6962 & 0.9368 & -10.589 & -11.374 & 0.0175 & 0.3934 \\
\hline $\mathrm{PE}(38: 4)$ & -10.939 & -10.070 & 0.0035 & 0.1599 & -11.357 & -11.633 & 0.5373 & 0.9350 & -11.414 & -11.728 & 0.4127 & 0.6537 \\
\hline PE(38:4) & -9.609 & -9.017 & 0.0160 & 0.2352 & -9.539 & -9.517 & 0.9447 & 0.9848 & -9.344 & -9.806 & 0.0822 & 0.3938 \\
\hline $\operatorname{PE}(38: 4)$ & -7.210 & -6.573 & 0.0035 & 0.1599 & -7.047 & -6.853 & 0.6839 & 0.9368 & -6.524 & -7.495 & 0.1328 & 0.4258 \\
\hline $\operatorname{PE}(38: 4 e)$ & -9.136 & -8.261 & 0.0200 & 0.2588 & -8.926 & -8.532 & 0.3945 & 0.9350 & -8.696 & -8.372 & 0.2022 & 0.4774 \\
\hline $\operatorname{PE}(38: 5 e)$ & -9.747 & -8.574 & 0.0140 & 0.2235 & -9.492 & -9.193 & 0.5034 & 0.9350 & -9.171 & -9.229 & 0.8136 & 0.9044 \\
\hline $\operatorname{PE}(38: 5 e)$ & -6.646 & -6.152 & 0.1071 & 0.4392 & -6.168 & -5.988 & 0.5859 & 0.9350 & -5.666 & -6.220 & 0.2577 & 0.5276 \\
\hline $\mathrm{PE}(\mathrm{p} 16: 0 / 18: 1)$ & -7.348 & -6.626 & 0.1309 & 0.4521 & -7.219 & -7.382 & 0.6600 & 0.9368 & -6.600 & -7.422 & 0.1808 & 0.4554 \\
\hline PE(p16:0/18:2) & -9.835 & -9.501 & 0.3035 & 0.6168 & -9.744 & -9.604 & 0.7228 & 0.9486 & -8.639 & -9.663 & 0.2221 & 0.4934 \\
\hline PI(18:0/20:4) & -7.118 & -6.378 & 0.0053 & 0.1693 & -6.651 & -6.427 & 0.5719 & 0.9350 & -6.192 & -6.721 & 0.2222 & 0.4934 \\
\hline PI(36:3) & -11.355 & -10.437 & 0.0203 & 0.2588 & -12.107 & -12.025 & 0.8482 & 0.9665 & -11.683 & -12.118 & 0.1609 & 0.4367 \\
\hline $\operatorname{PI}(36: 4)$ & -11.490 & -10.311 & 0.0081 & 0.1837 & -11.962 & -11.535 & 0.2597 & 0.9350 & -11.852 & -11.726 & 0.4783 & 0.7061 \\
\hline $\operatorname{PI}(38: 3)$ & -10.411 & -10.072 & 0.1602 & 0.4768 & -10.142 & -10.066 & 0.8244 & 0.9665 & -9.417 & -10.581 & 0.0884 & 0.3977 \\
\hline PI(38:5) & -10.003 & -9.110 & 0.0137 & 0.2235 & -10.066 & -9.768 & 0.5196 & 0.9350 & -9.655 & -10.216 & 0.3093 & 0.5752 \\
\hline $\operatorname{PI}(40: 5)$ & -10.853 & -9.854 & 0.0045 & 0.1659 & -11.381 & -10.616 & 0.1582 & 0.9350 & -10.615 & -11.219 & 0.4152 & 0.6537 \\
\hline $\operatorname{SM}(\mathrm{d} 18: 1 / 16: 0)$ & -8.518 & -8.388 & 0.6049 & 0.8169 & -7.889 & -8.185 & 0.4568 & 0.9350 & -7.335 & -8.370 & 0.0533 & 0.3934 \\
\hline unknown & -6.636 & -6.800 & 0.4451 & 0.7156 & -6.337 & -6.706 & 0.2277 & 0.9350 & -6.405 & -6.635 & 0.4554 & 0.6883 \\
\hline unknown & -5.729 & -4.567 & 0.0669 & 0.3863 & -5.793 & -5.791 & 0.9983 & 0.9985 & -6.170 & -6.322 & 0.6900 & 0.8411 \\
\hline unknown & -6.402 & -5.500 & 0.0854 & 0.4121 & -6.773 & -6.749 & 0.9690 & 0.9876 & -7.066 & -7.433 & 0.1045 & 0.4114 \\
\hline unknown & -6.695 & -6.315 & 0.3539 & 0.6476 & -6.034 & -5.830 & 0.5785 & 0.9350 & -6.476 & -5.990 & 0.4569 & 0.6888 \\
\hline unknown & -5.770 & -5.984 & 0.2840 & 0.6066 & -5.765 & -5.912 & 0.3668 & 0.9350 & -6.091 & -5.996 & 0.1102 & 0.4114 \\
\hline unknown & -6.156 & -6.347 & 0.3649 & 0.6488 & -5.378 & -5.508 & 0.4326 & 0.9350 & -5.865 & -5.478 & 0.4052 & 0.6510 \\
\hline unknown & -5.497 & -5.952 & 0.1684 & 0.4881 & -4.017 & -4.324 & 0.2331 & 0.9350 & -4.970 & -4.563 & 0.5364 & 0.7419 \\
\hline unknown & -6.062 & -6.334 & 0.1815 & 0.5054 & -6.054 & -6.220 & 0.3914 & 0.9350 & -6.359 & -6.255 & 0.3402 & 0.5963 \\
\hline unknown & -10.213 & -10.329 & 0.5273 & 0.7826 & -10.302 & -10.240 & 0.5493 & 0.9350 & -10.278 & -10.283 & 0.9756 & 0.9850 \\
\hline unknown & -10.211 & -10.397 & 0.3855 & 0.6645 & -10.430 & -10.262 & 0.1057 & 0.9350 & -10.486 & -10.414 & 0.4610 & 0.6916 \\
\hline unknown & -9.462 & -9.508 & 0.5761 & 0.8025 & -9.435 & -9.421 & 0.9002 & 0.9792 & -9.750 & -9.573 & 0.0789 & 0.3934 \\
\hline unknown & -6.906 & -6.094 & 0.1065 & 0.4392 & -7.281 & -6.784 & 0.4036 & 0.9350 & -7.316 & -7.143 & 0.3597 & 0.6109 \\
\hline unknown & -10.117 & -10.154 & 0.7216 & 0.8518 & -10.094 & -10.011 & 0.3577 & 0.9350 & -10.278 & -10.157 & 0.3662 & 0.6135 \\
\hline
\end{tabular}




\begin{tabular}{|c|c|c|c|c|c|c|c|c|c|c|c|c|}
\hline unknown & -10.073 & -10.052 & 0.8381 & 0.8868 & -10.092 & -9.994 & 0.2609 & 0.9350 & -10.162 & -10.104 & 0.5767 & 0.7717 \\
\hline unknown & -4.161 & -3.875 & 0.0773 & 0.4088 & -3.981 & -3.772 & 0.4493 & 0.9350 & -3.977 & -3.716 & 0.2140 & 0.4895 \\
\hline unknown & -7.620 & -7.477 & 0.5331 & 0.7826 & -7.338 & -7.076 & 0.4826 & 0.9350 & -7.328 & -7.381 & 0.7294 & 0.8591 \\
\hline unknown & -7.101 & -7.261 & 0.1939 & 0.5120 & -7.172 & -7.176 & 0.9760 & 0.9908 & -7.320 & -7.061 & 0.0651 & 0.3934 \\
\hline unknown & -6.117 & -6.268 & 0.5684 & 0.8025 & -5.855 & -6.000 & 0.2325 & 0.9350 & -6.403 & -6.036 & 0.4022 & 0.6502 \\
\hline unknown & -6.360 & -5.721 & 0.0906 & 0.4242 & -6.117 & -5.923 & 0.5807 & 0.9350 & -6.170 & -6.102 & 0.8634 & 0.9268 \\
\hline unknown & -6.168 & -6.206 & 0.7065 & 0.8449 & -6.154 & -6.052 & 0.4513 & 0.9350 & -6.188 & -6.202 & 0.8838 & 0.9361 \\
\hline unknown & -6.806 & -6.660 & 0.4733 & 0.7463 & -6.293 & -6.142 & 0.4763 & 0.9350 & -6.410 & -6.223 & 0.5118 & 0.7318 \\
\hline unknown & -6.151 & -5.852 & 0.2390 & 0.5582 & -6.425 & -6.723 & 0.4289 & 0.9350 & -7.070 & -6.897 & 0.4534 & 0.6883 \\
\hline unknown & -9.612 & -9.342 & 0.2135 & 0.5306 & -9.642 & -10.013 & 0.2008 & 0.9350 & -10.141 & -9.932 & 0.3648 & 0.6135 \\
\hline unknown & -10.108 & -10.052 & 0.5186 & 0.7826 & -10.137 & -10.054 & 0.5818 & 0.9350 & -10.256 & -10.063 & 0.3542 & 0.6051 \\
\hline unknown & -10.086 & -10.065 & 0.8577 & 0.8985 & -10.190 & -10.001 & 0.1749 & 0.9350 & -10.258 & -10.210 & 0.6333 & 0.8091 \\
\hline unknown & -10.054 & -10.017 & 0.7409 & 0.8557 & -10.066 & -9.970 & 0.4533 & 0.9350 & -10.329 & -10.108 & 0.0587 & 0.3934 \\
\hline unknown & -4.039 & -3.445 & 0.1238 & 0.4520 & -3.829 & -3.589 & 0.4335 & 0.9350 & -4.065 & -3.823 & 0.6238 & 0.8054 \\
\hline unknown & -4.421 & -4.312 & 0.5720 & 0.8025 & -3.788 & -3.804 & 0.9257 & 0.9810 & -4.090 & -3.801 & 0.3855 & 0.6356 \\
\hline unknown & -7.045 & -6.959 & 0.5835 & 0.8071 & -6.770 & -7.137 & 0.2640 & 0.9350 & -7.546 & -7.382 & 0.4354 & 0.6715 \\
\hline unknown & -7.948 & -7.817 & 0.4776 & 0.7488 & -8.065 & -8.337 & 0.0016 & 0.4951 & -8.475 & -8.114 & 0.2979 & 0.5685 \\
\hline unknown & -10.592 & -10.596 & 0.9890 & 0.9890 & -10.655 & -10.593 & 0.8072 & 0.9665 & -10.809 & -10.228 & 0.0490 & 0.3934 \\
\hline unknown & -10.213 & -10.099 & 0.6232 & 0.8186 & -10.322 & -10.489 & 0.3707 & 0.9350 & -10.477 & -10.234 & 0.2616 & 0.5276 \\
\hline unknown & -6.533 & -6.266 & 0.1796 & 0.5054 & -7.264 & -7.476 & 0.4830 & 0.9350 & -7.835 & -7.439 & 0.3413 & 0.5965 \\
\hline unknown & -9.219 & -8.860 & 0.2866 & 0.6073 & -10.327 & -10.625 & 0.4770 & 0.9350 & -10.879 & -10.201 & 0.3301 & 0.5854 \\
\hline unknown & -9.922 & -9.856 & 0.6018 & 0.8166 & -9.938 & -9.751 & 0.5432 & 0.9350 & -11.117 & -10.662 & 0.4905 & 0.7178 \\
\hline unknown & -10.100 & -10.102 & 0.9947 & 0.9947 & -10.149 & -10.073 & 0.4622 & 0.9350 & -10.201 & -10.093 & 0.5353 & 0.7419 \\
\hline unknown & -10.165 & -10.302 & 0.4787 & 0.7488 & -10.267 & -10.194 & 0.5452 & 0.9350 & -10.199 & -10.334 & 0.3947 & 0.6440 \\
\hline unknown & -10.146 & -10.150 & 0.9862 & 0.9862 & -10.173 & -10.119 & 0.6132 & 0.9350 & -10.265 & -10.165 & 0.4889 & 0.7173 \\
\hline unknown & -10.254 & -10.287 & 0.8166 & 0.8834 & -10.301 & -10.199 & 0.4218 & 0.9350 & -10.327 & -10.310 & 0.8997 & 0.9437 \\
\hline unknown & -10.066 & -10.208 & 0.4842 & 0.7533 & -10.182 & -10.075 & 0.1314 & 0.9350 & -10.527 & -10.321 & 0.2363 & 0.5053 \\
\hline unknown & -10.590 & -10.576 & 0.9565 & 0.9566 & -10.320 & -10.159 & 0.6693 & 0.9368 & -11.519 & -10.894 & 0.2882 & 0.5587 \\
\hline unknown & -5.610 & -5.597 & 0.9520 & 0.9520 & -5.409 & -5.441 & 0.8834 & 0.9721 & -5.399 & -5.414 & 0.9022 & 0.9437 \\
\hline unknown & -8.020 & -7.853 & 0.4527 & 0.7218 & -8.666 & -8.914 & 0.5473 & 0.9350 & -9.209 & -8.795 & 0.4518 & 0.6880 \\
\hline unknown & -9.808 & -11.319 & 0.0987 & 0.4291 & -10.585 & -9.386 & 0.2596 & 0.9350 & -10.758 & -11.483 & 0.4550 & 0.6883 \\
\hline unknown & -6.453 & -6.337 & 0.4347 & 0.7101 & -6.265 & -6.396 & 0.4296 & 0.9350 & -6.377 & -6.297 & 0.2255 & 0.4945 \\
\hline unknown & -8.862 & -8.876 & 0.8284 & 0.8855 & -8.916 & -8.848 & 0.4858 & 0.9350 & -9.197 & -9.007 & 0.1438 & 0.4361 \\
\hline unknown & -8.931 & -9.007 & 0.3754 & 0.6533 & -9.023 & -8.856 & 0.1256 & 0.9350 & -9.093 & -8.947 & 0.1843 & 0.4554 \\
\hline unknown & -9.310 & -9.262 & 0.6214 & 0.8186 & -9.284 & -9.254 & 0.8282 & 0.9665 & -9.036 & -9.284 & 0.0824 & 0.3938 \\
\hline unknown & -8.317 & -8.383 & 0.2949 & 0.6073 & -8.306 & -8.332 & 0.8157 & 0.9665 & -8.317 & -8.349 & 0.6964 & 0.8416 \\
\hline unknown & -8.941 & -8.891 & 0.5215 & 0.7826 & -8.978 & -8.897 & 0.5101 & 0.9350 & -9.323 & -9.074 & 0.0092 & 0.3934 \\
\hline unknown & -8.867 & -8.899 & 0.7353 & 0.8557 & -8.953 & -8.876 & 0.2781 & 0.9350 & -8.974 & -8.930 & 0.5827 & 0.7756 \\
\hline unknown & -10.290 & -10.274 & 0.8846 & 0.9024 & -10.311 & -10.227 & 0.4173 & 0.9350 & -10.262 & -10.264 & 0.9879 & 0.9895 \\
\hline unknown & -10.392 & -10.352 & 0.5279 & 0.7826 & -10.259 & -10.248 & 0.9156 & 0.9804 & -10.324 & -10.294 & 0.5775 & 0.7717 \\
\hline unknown & -10.239 & -10.253 & 0.8263 & 0.8855 & -10.289 & -10.236 & 0.5545 & 0.9350 & -10.558 & -10.308 & 0.0459 & 0.3934 \\
\hline unknown & -10.175 & -10.185 & 0.8641 & 0.8985 & -10.254 & -10.090 & 0.0951 & 0.9350 & -10.452 & -10.297 & 0.1890 & 0.4597 \\
\hline unknown & -10.178 & -10.189 & 0.8891 & 0.9024 & -10.285 & -10.155 & 0.2169 & 0.9350 & -10.478 & -10.256 & 0.1259 & 0.4215 \\
\hline unknown & -9.877 & -9.848 & 0.8729 & 0.8985 & -9.994 & -10.351 & 0.0015 & 0.4951 & -10.632 & -10.124 & 0.0954 & 0.4048 \\
\hline unknown & -9.276 & -9.312 & 0.8881 & 0.9024 & -9.675 & -9.979 & 0.4855 & 0.9350 & -10.304 & -10.242 & 0.8460 & 0.9194 \\
\hline unknown & -9.766 & -9.754 & 0.9515 & 0.9515 & -9.878 & -9.821 & 0.3179 & 0.9350 & -9.765 & -9.812 & 0.7756 & 0.8865 \\
\hline unknown & -9.216 & -9.774 & 0.0766 & 0.4088 & -8.933 & -8.824 & 0.5562 & 0.9350 & -8.974 & -9.645 & 0.1089 & 0.4114 \\
\hline unknown & -10.755 & -11.158 & 0.6914 & 0.8372 & -10.992 & -10.552 & 0.6618 & 0.9368 & -10.952 & -10.216 & 0.1286 & 0.4215 \\
\hline unknown & -8.082 & -7.874 & 0.2435 & 0.5591 & -8.837 & -9.057 & 0.4461 & 0.9350 & -9.362 & -8.908 & 0.3091 & 0.5752 \\
\hline unknown & -5.610 & -5.564 & 0.5892 & 0.8112 & -5.576 & -5.553 & 0.8638 & 0.9665 & -5.572 & -5.566 & 0.9535 & 0.9708 \\
\hline unknown & -3.676 & -3.512 & 0.1766 & 0.5018 & -3.650 & -3.436 & 0.4241 & 0.9350 & -3.473 & -3.616 & 0.3768 & 0.6277 \\
\hline unknown & -9.455 & -9.522 & 0.7371 & 0.8557 & -9.587 & -9.510 & 0.5273 & 0.9350 & -9.500 & -9.531 & 0.8639 & 0.9268 \\
\hline unknown & -3.926 & -3.948 & 0.6284 & 0.8224 & -3.934 & -3.861 & 0.4116 & 0.9350 & -3.967 & -3.926 & 0.6168 & 0.8041 \\
\hline unknown & -10.655 & -10.973 & 0.3539 & 0.6476 & -10.417 & -11.212 & 0.0143 & 0.9350 & -11.599 & -11.670 & 0.9046 & 0.9437 \\
\hline unknown & -9.199 & -9.067 & 0.5211 & 0.7826 & -9.800 & -10.088 & 0.4931 & 0.9350 & -10.346 & -10.241 & 0.8051 & 0.8990 \\
\hline unknown & -10.485 & -9.988 & 0.1365 & 0.4557 & -10.275 & -9.863 & 0.3296 & 0.9350 & -10.241 & -10.365 & 0.7886 & 0.8911 \\
\hline unknown & -6.443 & -6.892 & 0.0520 & 0.3484 & -6.381 & -6.213 & 0.3444 & 0.9350 & -6.532 & -6.786 & 0.1468 & 0.4361 \\
\hline unknown & -5.541 & -5.544 & 0.9856 & 0.9856 & -5.721 & -5.519 & 0.2898 & 0.9350 & -5.506 & -5.477 & 0.8081 & 0.9007 \\
\hline unknown & -9.191 & -8.791 & 0.1383 & 0.4558 & -9.198 & -9.063 & 0.7243 & 0.9486 & -9.852 & -10.019 & 0.8909 & 0.9383 \\
\hline unknown & -7.164 & -7.329 & 0.1850 & 0.5057 & -7.178 & -7.265 & 0.6811 & 0.9368 & -7.134 & -7.318 & 0.4485 & 0.6847 \\
\hline unknown & -10.167 & -9.266 & 0.2788 & 0.6061 & -9.927 & -9.689 & 0.8134 & 0.9665 & -8.751 & -9.709 & 0.2490 & 0.5187 \\
\hline unknown & -4.203 & -4.175 & 0.6957 & 0.8372 & -4.200 & -4.153 & 0.5885 & 0.9350 & -4.251 & -4.106 & 0.0723 & 0.3934 \\
\hline unknown & -9.794 & -9.888 & 0.2419 & 0.5588 & -9.959 & -9.792 & 0.2143 & 0.9350 & -10.139 & -9.912 & 0.0684 & 0.3934 \\
\hline unknown & -9.950 & -9.940 & 0.8988 & 0.9024 & -9.990 & -9.827 & 0.1766 & 0.9350 & -10.227 & -10.000 & 0.0724 & 0.3934 \\
\hline unknown & -9.868 & -9.888 & 0.8160 & 0.8834 & -9.963 & -9.840 & 0.2521 & 0.9350 & -10.100 & -9.914 & 0.0337 & 0.3934 \\
\hline unknown & -7.175 & -6.679 & 0.1603 & 0.4768 & -6.914 & -6.541 & 0.3414 & 0.9350 & -7.221 & -6.832 & 0.5029 & 0.7255 \\
\hline unknown & -9.381 & -9.404 & 0.8833 & 0.9024 & -9.477 & -9.370 & 0.3391 & 0.9350 & -9.602 & -9.442 & 0.0521 & 0.3934 \\
\hline unknown & -4.115 & -2.900 & 0.1457 & 0.4647 & -3.771 & -3.192 & 0.3981 & 0.9350 & -4.184 & -3.607 & 0.5354 & 0.7419 \\
\hline unknown & -8.443 & -8.471 & 0.8137 & 0.8834 & -8.499 & -8.439 & 0.4962 & 0.9350 & -8.433 & -8.396 & 0.7415 & 0.8665 \\
\hline unknown & -9.605 & -9.675 & 0.6162 & 0.8186 & -9.732 & -9.670 & 0.6497 & 0.9368 & -9.704 & -9.589 & 0.3032 & 0.5731 \\
\hline unknown & -3.846 & -3.982 & 0.5030 & 0.7699 & -3.888 & -3.908 & 0.9074 & 0.9804 & -3.758 & -3.795 & 0.8230 & 0.9103 \\
\hline unknown & -5.398 & -5.263 & 0.6860 & 0.8363 & -5.027 & -5.605 & 0.1212 & 0.9350 & -5.412 & -5.393 & 0.9641 & 0.9787 \\
\hline unknown & -8.730 & -8.702 & 0.7109 & 0.8471 & -8.661 & -8.670 & 0.9235 & 0.9804 & -8.938 & -8.819 & 0.1163 & 0.4119 \\
\hline unknown & -9.814 & -9.793 & 0.8072 & 0.8834 & -9.820 & -9.815 & 0.9550 & 0.9848 & -9.816 & -9.802 & 0.9122 & 0.946 \\
\hline
\end{tabular}




\begin{tabular}{|c|c|c|c|c|c|c|c|c|c|c|c|c|}
\hline unknown & -9.564 & -9.570 & 0.9539 & 0.9540 & -9.591 & -9.514 & 0.5361 & 0.9350 & -9.529 & -9.596 & 0.5646 & 0.7676 \\
\hline unknown & -10.464 & -10.414 & 0.5927 & 0.8120 & -10.423 & -10.307 & 0.4961 & 0.9350 & -10.349 & -10.416 & 0.4586 & 0.6896 \\
\hline unknown & -9.692 & -8.842 & 0.0826 & 0.4121 & -9.985 & -9.546 & 0.0149 & 0.9350 & -9.932 & -9.493 & 0.0526 & 0.3934 \\
\hline unknown & -9.146 & -9.213 & 0.6228 & 0.8186 & -9.262 & -9.338 & 0.6269 & 0.9350 & -9.336 & -9.297 & 0.8404 & 0.9167 \\
\hline unknown & -8.201 & -8.098 & 0.6849 & 0.8363 & -8.269 & -8.300 & 0.8819 & 0.9721 & -8.979 & -8.141 & 0.0240 & 0.3934 \\
\hline unknown & -9.109 & -8.313 & 0.0198 & 0.2588 & -8.831 & -8.257 & 0.2932 & 0.9350 & -9.185 & -9.019 & 0.6940 & 0.8411 \\
\hline unknown & -9.694 & -9.766 & 0.6375 & 0.8242 & -9.863 & -9.742 & 0.3727 & 0.9350 & -9.773 & -9.803 & 0.8540 & 0.9229 \\
\hline unknown & -10.255 & -10.135 & 0.1343 & 0.4542 & -10.281 & -10.088 & 0.1335 & 0.9350 & -10.631 & -10.233 & 0.0195 & 0.3934 \\
\hline unknown & -10.288 & -10.188 & 0.0965 & 0.4262 & -10.299 & -10.160 & 0.2139 & 0.9350 & -10.670 & -10.384 & 0.0112 & 0.3934 \\
\hline unknown & -8.078 & -7.407 & 0.4662 & 0.7372 & -6.213 & -8.015 & 0.1774 & 0.9350 & -6.866 & -6.231 & 0.0880 & 0.3977 \\
\hline unknown & -10.471 & -10.543 & 0.7716 & 0.8745 & -10.605 & -10.575 & 0.8685 & 0.9665 & -10.575 & -10.218 & 0.1891 & 0.4597 \\
\hline unknown & -10.558 & -9.955 & 0.0331 & 0.2953 & -10.822 & -10.989 & 0.6860 & 0.9368 & -10.301 & -10.828 & 0.1561 & 0.4367 \\
\hline unknown & -10.101 & -10.067 & 0.9009 & 0.9024 & -10.014 & -10.146 & 0.5687 & 0.9350 & -10.241 & -10.115 & 0.5166 & 0.7347 \\
\hline unknown & -5.286 & -5.264 & 0.8177 & 0.8834 & -5.125 & -5.172 & 0.6588 & 0.9368 & -5.277 & -5.251 & 0.7475 & 0.8702 \\
\hline unknown & -10.898 & -11.032 & 0.5375 & 0.7870 & -10.996 & -10.983 & 0.9489 & 0.9848 & -11.004 & -10.707 & 0.1172 & 0.4119 \\
\hline unknown & -10.793 & -10.425 & 0.2331 & 0.5513 & -10.782 & -11.188 & 0.3688 & 0.9350 & -10.410 & -10.866 & 0.1832 & 0.4554 \\
\hline unknown & -10.873 & -10.443 & 0.1315 & 0.4521 & -10.910 & -11.149 & 0.4392 & 0.9350 & -10.515 & -11.149 & 0.2278 & 0.4976 \\
\hline unknown & -9.901 & -9.835 & 0.4632 & 0.7366 & -9.899 & -9.795 & 0.4327 & 0.9350 & -10.034 & -9.946 & 0.6237 & 0.8054 \\
\hline unknown & -9.786 & -9.826 & 0.6469 & 0.8253 & -9.993 & -9.825 & 0.2849 & 0.9350 & -10.248 & -10.029 & 0.0083 & 0.3934 \\
\hline unknown & -9.784 & -9.799 & 0.8655 & 0.8985 & -9.929 & -9.810 & 0.3605 & 0.9350 & -10.167 & -9.918 & 0.0245 & 0.3934 \\
\hline unknown & -9.903 & -9.871 & 0.7489 & 0.8597 & -9.908 & -9.884 & 0.8268 & 0.9665 & -10.034 & -9.962 & 0.6764 & 0.8358 \\
\hline unknown & -9.624 & -9.981 & 0.3319 & 0.6346 & -9.281 & -9.840 & 0.1981 & 0.9350 & -9.260 & -9.835 & 0.2625 & 0.5276 \\
\hline unknown & -10.303 & -10.193 & 0.6937 & 0.8372 & -10.156 & -10.300 & 0.6188 & 0.9350 & -9.799 & -10.008 & 0.2669 & 0.5330 \\
\hline unknown & -10.607 & -10.115 & 0.0835 & 0.4121 & -10.870 & -10.629 & 0.5632 & 0.9350 & -10.920 & -11.133 & 0.4163 & 0.6538 \\
\hline unknown & -10.186 & -9.955 & 0.6957 & 0.8372 & -10.143 & -10.619 & 0.2941 & 0.9350 & -9.724 & -9.920 & 0.6525 & 0.8215 \\
\hline unknown & -10.993 & -10.096 & 0.1936 & 0.5120 & -10.861 & -11.103 & 0.6683 & 0.9368 & -10.112 & -11.124 & 0.1656 & 0.4379 \\
\hline unknown & -5.649 & -5.574 & 0.4023 & 0.6782 & -5.604 & -5.521 & 0.3171 & 0.9350 & -5.669 & -5.618 & 0.5565 & 0.7592 \\
\hline unknown & -8.452 & -8.272 & 0.8127 & 0.8834 & -8.635 & -9.309 & 0.3308 & 0.9350 & -8.014 & -7.933 & 0.8846 & 0.9361 \\
\hline unknown & -10.636 & -10.399 & 0.3090 & 0.6223 & -10.615 & -10.424 & 0.4658 & 0.9350 & -10.264 & -10.698 & 0.1163 & 0.4119 \\
\hline unknown & -10.177 & -10.060 & 0.7630 & 0.8707 & -10.222 & -10.353 & 0.6020 & 0.9350 & -10.105 & -10.139 & 0.8860 & 0.9361 \\
\hline unknown & -11.589 & -11.275 & 0.5089 & 0.7749 & -11.203 & -11.541 & 0.4908 & 0.9350 & -10.573 & -11.623 & 0.1586 & 0.4367 \\
\hline unknown & -10.571 & -10.312 & 0.2498 & 0.5667 & -10.668 & -10.531 & 0.6640 & 0.9368 & -10.256 & -10.629 & 0.3191 & 0.5761 \\
\hline unknown & -11.165 & -10.481 & 0.1907 & 0.5092 & -11.091 & -11.362 & 0.5725 & 0.9350 & -10.871 & -11.506 & 0.4040 & 0.6510 \\
\hline unknown & -9.724 & -9.182 & 0.1694 & 0.4885 & -9.514 & -9.816 & 0.4620 & 0.9350 & -9.173 & -9.865 & 0.1802 & 0.4554 \\
\hline unknown & -9.473 & -9.605 & 0.1307 & 0.4521 & -9.672 & -9.496 & 0.1384 & 0.9350 & -9.604 & -9.571 & 0.8021 & 0.8990 \\
\hline unknown & -9.546 & -9.147 & 0.0664 & 0.3863 & -9.616 & -9.408 & 0.5115 & 0.9350 & -9.043 & -9.676 & 0.1246 & 0.4215 \\
\hline unknown & -8.703 & -8.452 & 0.1456 & 0.4647 & -8.567 & -8.543 & 0.9140 & 0.9804 & -8.185 & -8.593 & 0.1463 & 0.4361 \\
\hline unknown & -7.494 & -7.479 & 0.9008 & 0.9024 & -7.490 & -7.406 & 0.3232 & 0.9350 & -7.383 & -7.533 & 0.3269 & 0.5832 \\
\hline unknown & -6.938 & -6.913 & 0.7544 & 0.8626 & -7.015 & -6.914 & 0.3849 & 0.9350 & -7.176 & -7.000 & 0.0287 & 0.3934 \\
\hline unknown & -8.557 & -8.501 & 0.4429 & 0.7141 & -8.515 & -8.317 & 0.2157 & 0.9350 & -8.579 & -8.482 & 0.5048 & 0.7262 \\
\hline unknown & -8.545 & -8.499 & 0.5764 & 0.8025 & -8.622 & -8.541 & 0.3427 & 0.9350 & -8.509 & -8.533 & 0.7870 & 0.8911 \\
\hline unknown & -7.448 & -7.447 & 0.9759 & 0.9759 & -7.325 & -7.368 & 0.8539 & 0.9665 & -7.653 & -7.531 & 0.1388 & 0.4359 \\
\hline unknown & -10.052 & -10.023 & 0.7748 & 0.8755 & -10.107 & -10.087 & 0.8700 & 0.9665 & -9.995 & -10.129 & 0.2127 & 0.4895 \\
\hline unknown & -8.640 & -8.680 & 0.5259 & 0.7826 & -8.739 & -8.695 & 0.7385 & 0.9556 & -8.642 & -8.713 & 0.2770 & 0.5440 \\
\hline unknown & -9.057 & -9.048 & 0.9049 & 0.9049 & -9.138 & -8.945 & 0.1714 & 0.9350 & -9.118 & -9.052 & 0.5767 & 0.7717 \\
\hline unknown & -9.388 & -9.293 & 0.2956 & 0.6073 & -9.271 & -9.156 & 0.5029 & 0.9350 & -9.154 & -9.248 & 0.4223 & 0.6573 \\
\hline unknown & -6.643 & -6.691 & 0.8025 & 0.8834 & -6.735 & -6.552 & 0.4008 & 0.9350 & -7.003 & -6.598 & 0.0179 & 0.3934 \\
\hline unknown & -9.887 & -9.721 & 0.2904 & 0.6073 & -9.791 & -9.339 & 0.2392 & 0.9350 & -9.732 & -9.984 & 0.3150 & 0.5752 \\
\hline unknown & -9.235 & -9.226 & 0.9145 & 0.9145 & -9.125 & -9.073 & 0.5862 & 0.9350 & -9.168 & -9.196 & 0.8478 & 0.9195 \\
\hline unknown & -8.108 & -8.085 & 0.9005 & 0.9024 & -8.581 & -8.318 & 0.6070 & 0.9350 & -7.640 & -8.207 & 0.1019 & 0.4106 \\
\hline unknown & -8.111 & -7.661 & 0.1664 & 0.4848 & -7.904 & -7.701 & 0.6120 & 0.9350 & -7.317 & -8.060 & 0.0788 & 0.3934 \\
\hline unknown & -10.064 & -10.056 & 0.9366 & 0.9366 & -10.122 & -10.017 & 0.4170 & 0.9350 & -10.484 & -10.288 & 0.0454 & 0.3934 \\
\hline unknown & -10.061 & -9.993 & 0.3728 & 0.6533 & -10.042 & -10.077 & 0.8073 & 0.9665 & -10.275 & -10.053 & 0.0425 & 0.3934 \\
\hline unknown & -7.234 & -7.463 & 0.2755 & 0.6013 & -7.660 & -7.447 & 0.3657 & 0.9350 & -8.080 & -7.495 & 0.0458 & 0.3934 \\
\hline unknown & -9.482 & -8.937 & 0.2088 & 0.5278 & -9.151 & -9.148 & 0.9939 & 0.9985 & -8.462 & -9.304 & 0.1552 & 0.4367 \\
\hline unknown & -7.118 & -7.314 & 0.4004 & 0.6782 & -7.667 & -6.973 & 0.1686 & 0.9350 & -7.373 & -7.636 & 0.3182 & 0.5761 \\
\hline unknown & -9.075 & -9.315 & 0.1615 & 0.4780 & -9.163 & -9.019 & 0.3887 & 0.9350 & -9.395 & -9.130 & 0.1220 & 0.4205 \\
\hline unknown & -6.148 & -6.037 & 0.2466 & 0.5639 & -6.069 & -5.983 & 0.4298 & 0.9350 & -6.121 & -6.109 & 0.9218 & 0.9475 \\
\hline unknown & -9.313 & -9.044 & 0.6744 & 0.8363 & -9.305 & -9.755 & 0.2800 & 0.9350 & -8.484 & -8.736 & 0.6506 & 0.8215 \\
\hline unknown & -9.344 & -9.219 & 0.8481 & 0.8933 & -9.500 & -9.705 & 0.5181 & 0.9350 & -8.900 & -8.974 & 0.8865 & 0.9361 \\
\hline unknown & -10.385 & -9.791 & 0.0100 & 0.1949 & -10.488 & -10.300 & 0.6792 & 0.9368 & -9.780 & -10.610 & 0.1517 & 0.4361 \\
\hline unknown & -10.722 & -10.097 & 0.1894 & 0.5092 & -10.379 & -10.332 & 0.8931 & 0.9758 & -9.790 & -10.575 & 0.2412 & 0.5121 \\
\hline unknown & -8.735 & -8.470 & 0.7365 & 0.8557 & -8.853 & -9.513 & 0.3164 & 0.9350 & -8.291 & -8.313 & 0.9677 & 0.9807 \\
\hline unknown & -9.401 & -9.382 & 0.8337 & 0.8862 & -9.432 & -9.365 & 0.5964 & 0.9350 & -9.739 & -9.585 & 0.2076 & 0.4828 \\
\hline unknown & -10.060 & -9.989 & 0.4031 & 0.6782 & -10.204 & -10.004 & 0.2108 & 0.9350 & -10.205 & -10.161 & 0.6199 & 0.8041 \\
\hline unknown & -9.927 & -9.943 & 0.8705 & 0.8985 & -9.952 & -9.880 & 0.6041 & 0.9350 & -10.174 & -10.028 & 0.0255 & 0.3934 \\
\hline unknown & -10.157 & -10.091 & 0.3627 & 0.6487 & -10.113 & -10.046 & 0.6121 & 0.9350 & -10.325 & -10.226 & 0.3336 & 0.5882 \\
\hline unknown & -10.132 & -10.047 & 0.3520 & 0.6476 & -10.070 & -10.033 & 0.7268 & 0.9486 & -10.504 & -10.261 & 0.0062 & 0.3934 \\
\hline unknown & -9.998 & -9.556 & 0.2334 & 0.5513 & -9.681 & -9.587 & 0.8391 & 0.9665 & -9.330 & -10.075 & 0.0434 & 0.3934 \\
\hline unknown & -9.654 & -9.681 & 0.9063 & 0.9063 & -9.781 & -9.751 & 0.8569 & 0.9665 & -10.194 & -9.882 & 0.0140 & 0.3934 \\
\hline unknown & -7.722 & -7.162 & 0.5775 & 0.8025 & -5.765 & -7.436 & 0.2115 & 0.9350 & -6.390 & -5.660 & 0.1525 & 0.4361 \\
\hline unknown & -9.840 & -9.589 & 0.0983 & 0.4291 & -9.750 & -9.586 & 0.5150 & 0.9350 & -9.497 & -9.778 & 0.2896 & 0.5597 \\
\hline unknown & -7.935 & -7.833 & 0.6858 & 0.8363 & -7.821 & -7.930 & 0.6918 & 0.9368 & -7.664 & -8.034 & 0.1906 & 0.4598 \\
\hline unknown & -9.997 & -9.562 & 0.1422 & 0.4613 & -10.050 & -10.190 & 0.7307 & 0.9507 & -10.096 & -10.278 & 0.6026 & 0.791 \\
\hline
\end{tabular}




\begin{tabular}{|c|c|c|c|c|c|c|c|c|c|c|c|c|}
\hline unknown & -10.775 & -10.290 & 0.2831 & 0.6066 & -10.984 & -10.596 & 0.3610 & 0.9350 & -11.907 & -10.844 & 0.0463 & 0.3934 \\
\hline unknown & -12.014 & -11.803 & 0.5925 & 0.8120 & -11.536 & -11.486 & 0.9408 & 0.9848 & -10.721 & -12.211 & 0.0495 & 0.3934 \\
\hline unknown & -9.166 & -8.961 & 0.4291 & 0.7050 & -9.000 & -8.756 & 0.6140 & 0.9350 & -8.289 & -9.076 & 0.1089 & 0.411 \\
\hline unknown & -10.158 & -9.509 & 0.2279 & 0.5509 & -10.131 & -9.949 & 0.7419 & 0.9580 & -11.502 & -9.448 & 0.0436 & 0.393 \\
\hline unknown & -10.127 & -9.256 & 0.0808 & 0.4121 & -9.769 & -9.745 & 0.9597 & 0.9848 & -9.160 & -10.246 & 0.0988 & 0.41 \\
\hline unknown & -11.598 & -11.406 & 0.6341 & 0.8224 & -11.433 & -11.073 & 0.5906 & 0.9350 & -10.225 & -11.753 & 0.0705 & 0.393 \\
\hline unknown & -10.319 & -10.202 & 0.7114 & 0.8471 & -9.675 & -9.838 & 0.6805 & 0.9368 & -9.294 & -10.417 & 0.0218 & 0.39 \\
\hline unknown & -5.784 & -5.821 & 0.5717 & 0.8025 & -5.881 & -5.731 & 0.1795 & 0.9350 & -5.895 & -5.785 & 0.2447 & 0.51 \\
\hline unknown & -8.637 & -8.660 & 0.7815 & 0.8762 & -8.720 & -8.545 & 0.2013 & 0.9350 & -8.740 & -8.657 & 0.4470 & 0.68 \\
\hline unknown & -10.246 & -10.332 & 0.6838 & 0.8363 & -10.234 & -10.368 & 0.6240 & 0.9350 & -10.248 & -10.256 & 0.9753 & 0.98 \\
\hline unknown & -8.474 & -7.758 & 0.0626 & 0.3790 & -7.972 & -7.525 & 0.3555 & 0.9350 & -7.375 & -7.947 & 0.2143 & 0.48 \\
\hline unknown & -7.271 & -6.837 & 0.1312 & 0.4521 & -6.897 & -6.949 & 0.8794 & 0.9712 & -6.359 & -7.292 & 0.0592 & 0.393 \\
\hline unknown & -4.229 & -4.225 & 0.9711 & 0.9711 & -4.479 & -4.321 & 0.4588 & 0.9350 & -4.293 & -4.528 & 0.3435 & $0.59^{\circ}$ \\
\hline unknown & -10.935 & -10.522 & 0.3574 & 0.6476 & -10.360 & -10.534 & 0.7316 & 0.9507 & -9.534 & -10.994 & 0.0495 & 0.39 \\
\hline unknown & -3.517 & -3.585 & 0.3372 & 0.6387 & -3.561 & -3.569 & 0.9498 & 0.9848 & -3.529 & -3.571 & 0.6884 & 0.84 \\
\hline unknown & -6.049 & -6.173 & 0.6158 & 0.8186 & -6.037 & -5.810 & 0.2598 & 0.9350 & -5.975 & -6.390 & 0.0829 & 0.39 \\
\hline unknown & -7.664 & -7.593 & 0.8695 & 0.8985 & -7.606 & -7.152 & 0.1036 & 0.9350 & -7.491 & -7.753 & 0.3446 & $0.59^{\prime}$ \\
\hline unknown & -5.366 & -5.293 & 0.1583 & 0.4757 & -5.406 & -5.330 & 0.4918 & 0.9350 & -5.375 & -5.343 & 0.6946 & 0.84 \\
\hline unknown & -10.005 & -9.233 & 0.1398 & 0.4558 & -9.290 & -9.025 & 0.6168 & 0.9350 & -8.736 & -9.355 & 0.3064 & 0.57 \\
\hline unknown & -10.444 & -9.999 & 0.4653 & 0.7372 & -10.755 & -10.658 & 0.8049 & 0.9665 & -10.061 & -10.177 & 0.7959 & 0.89 \\
\hline unknown & -10.152 & -10.139 & 0.8866 & 0.9024 & -10.240 & -10.260 & 0.9185 & 0.9804 & -10.037 & -10.232 & 0.3493 & 0.59 \\
\hline unknown & -14.239 & -12.310 & 0.1144 & 0.4451 & -12.379 & -13.184 & 0.3215 & 0.9350 & -13.076 & -13.518 & 0.4684 & 0.697 \\
\hline unknown & -10.943 & -10.519 & 0.0776 & 0.4088 & -10.790 & -10.895 & 0.6428 & 0.9368 & -10.551 & -10.982 & 0.0400 & 0.393 \\
\hline unknown & -10.331 & -9.902 & 0.0439 & 0.3361 & -10.416 & -10.258 & 0.6736 & 0.9368 & -10.095 & -10.710 & 0.1654 & 0.437 \\
\hline unknown & -10.720 & -10.296 & 0.1264 & 0.4521 & -10.648 & -10.582 & 0.9042 & 0.9804 & -10.422 & -11.103 & 0.0955 & 0.40 \\
\hline unknown & -8.309 & -8.280 & 0.5600 & 0.7959 & -8.414 & -8.233 & 0.2186 & 0.9350 & -8.256 & -8.316 & 0.5770 & 0.771 \\
\hline unknown & -8.097 & -7.830 & 0.2804 & 0.6066 & -8.082 & -7.936 & 0.4004 & 0.9350 & -8.041 & -7.779 & 0.4448 & 0.68 \\
\hline unknown & -8.162 & -8.137 & 0.8043 & 0.8834 & -7.970 & -7.957 & 0.8659 & 0.9665 & -8.209 & -8.113 & 0.1720 & 0.445 \\
\hline unknown & -8.860 & -8.820 & 0.5134 & 0.7776 & -8.908 & -8.859 & 0.6957 & 0.9368 & -8.798 & -8.816 & 0.9036 & 0.943 \\
\hline unknown & -9.986 & -9.857 & 0.1999 & 0.5146 & -9.971 & -9.921 & 0.6758 & 0.9368 & -10.559 & -10.233 & 0.0340 & 0.393 \\
\hline unknown & -9.518 & -9.487 & 0.7980 & 0.8834 & -9.541 & -9.363 & 0.1400 & 0.9350 & -9.741 & -9.637 & 0.0112 & 0.393 \\
\hline unknown & -9.850 & -9.809 & 0.6633 & 0.8350 & -9.757 & -9.806 & 0.6991 & 0.9368 & -10.293 & -10.097 & 0.0149 & 0.393 \\
\hline unknown & -8.899 & -8.814 & 0.2625 & 0.5817 & -8.873 & -8.795 & 0.5982 & 0.9350 & -8.872 & -8.873 & 0.9857 & 0.985 \\
\hline unknown & -8.702 & -8.699 & 0.9828 & 0.9828 & -8.759 & -8.574 & 0.1723 & 0.9350 & -8.744 & -8.746 & 0.9829 & 0.987 \\
\hline unknown & -7.602 & -7.753 & 0.1990 & 0.5145 & -7.559 & -7.422 & 0.2990 & 0.9350 & -7.676 & -7.831 & 0.2744 & 0.54 \\
\hline unknown & -6.987 & -6.981 & 0.9495 & 0.9495 & -7.037 & -6.916 & 0.2821 & 0.9350 & -7.192 & -7.101 & 0.1129 & 0.411 \\
\hline unknown & -9.579 & -9.635 & 0.5575 & 0.7959 & -9.676 & -9.606 & 0.5820 & 0.9350 & -9.631 & -9.640 & 0.9193 & 0.947 \\
\hline unknown & -6.405 & -6.616 & 0.3947 & 0.6742 & -6.285 & -6.486 & 0.4819 & 0.9350 & -6.258 & -6.497 & 0.4300 & 0.664 \\
\hline unknown & -10.362 & -10.280 & 0.6224 & 0.8186 & -10.402 & -10.251 & 0.4315 & 0.9350 & -10.503 & -10.419 & 0.6026 & 0.791 \\
\hline unknown & -9.713 & -9.624 & 0.3159 & 0.6296 & -9.656 & -9.552 & 0.5383 & 0.9350 & -9.694 & -9.724 & 0.8277 & 0.910 \\
\hline unknown & -7.977 & -7.912 & 0.4522 & 0.7218 & -8.007 & -7.906 & 0.4558 & 0.9350 & -8.081 & -7.989 & 0.1506 & 0.436 \\
\hline unknown & -8.532 & -8.601 & 0.3735 & 0.6533 & -8.525 & -8.636 & 0.3698 & 0.9350 & -8.723 & -8.554 & 0.3140 & 0.575 \\
\hline unknown & -9.911 & -9.889 & 0.8700 & 0.8985 & -9.927 & -9.859 & 0.5007 & 0.9350 & -9.926 & -9.969 & 0.7154 & 0.850 \\
\hline unknown & -9.693 & -10.029 & 0.4213 & 0.7010 & -9.319 & -9.834 & 0.2005 & 0.9350 & -9.320 & -9.790 & 0.3333 & 0.588 \\
\hline unknown & -11.598 & -10.997 & 0.1100 & 0.4448 & -11.420 & -10.917 & 0.3528 & 0.9350 & -10.828 & -11.676 & 0.0417 & 0.393 \\
\hline unknown & -10.421 & -10.176 & 0.2925 & 0.6073 & -10.338 & -10.251 & 0.7507 & 0.9605 & -10.121 & -10.540 & 0.1630 & 0.436 \\
\hline unknown & -9.402 & -9.361 & 0.6528 & 0.8272 & -9.382 & -9.347 & 0.7534 & 0.9605 & -9.521 & -9.420 & 0.1431 & 0.436 \\
\hline unknown & -9.540 & -9.520 & 0.8121 & 0.8834 & -9.605 & & 0.5560 & 0.9350 & -9.864 & -9.668 & 0.0585 & \\
\hline unknown & -9.916 & -10.051 & 0.1390 & 0.4558 & -9.999 & -10.010 & 0.9383 & 0.9848 & -10.154 & -10.049 & 0.5132 & 0.731 \\
\hline unknown & -10.330 & -10.255 & 0.7722 & 0.8745 & -10.163 & -10.459 & 0.2795 & 0.9350 & -9.863 & -10.192 & 0.1881 & 0.459 \\
\hline unknown & -9.512 & -8.733 & 0.0067 & 0.1751 & -9.494 & -9.007 & 0.4024 & 0.9350 & -8.895 & -9.861 & 0.1126 & 0.411 \\
\hline unknown & -9.016 & -8.789 & 0.3607 & 0.6487 & -8.658 & -8.628 & 0.9403 & 0.9848 & -8.468 & -9.063 & 0.0782 & 0.393 \\
\hline unknown & -9.290 & -8.967 & 0.3062 & 0.6190 & -9.405 & -9.533 & 0.7640 & 0.9658 & -10.573 & -9.710 & 0.0557 & 0.393 \\
\hline unknown & -10.767 & -10.190 & 0.1515 & 0.4702 & -10.191 & -10.128 & 0.8932 & 0.9758 & -9.484 & -10.495 & 0.0606 & 0.393 \\
\hline unknown & -8.147 & -7.625 & 0.2597 & 0.5816 & -8.235 & -8.234 & 0.9976 & 0.9985 & -9.721 & -8.060 & 0.0508 & 0.393 \\
\hline unknown & -8.619 & -8.166 & 0.2958 & 0.6073 & -8.792 & -8.942 & 0.7994 & 0.9665 & -10.104 & -8.682 & 0.0592 & 0.393 \\
\hline unknown & -6.411 & -6.288 & 0.1816 & 0.5054 & -6.317 & -6.361 & 0.6279 & 0.9350 & -6.454 & -6.359 & 0.4006 & \\
\hline unknown & -8.095 & -7.798 & 0.3580 & 0.6476 & -7.771 & -7.768 & 0.9916 & 0.9985 & -7.432 & -7.838 & 0.2245 & 0.49 \\
\hline unknown & -7.911 & -7.641 & 0.3173 & 0.6302 & -7.635 & -7.965 & 0.2499 & 0.9350 & -7.467 & -7.879 & 0.0487 & 0.393 \\
\hline unknown & -7.510 & -6.951 & 0.1345 & 0.4542 & -7.945 & -7.367 & 0.3365 & 0.9350 & -9.483 & -7.952 & 0.0266 & 0.393 \\
\hline unknown & -6.718 & -6.337 & 0.0526 & 0.3484 & -6.776 & -6.605 & 0.6202 & 0.9350 & -6.486 & -6.870 & 0.1825 & 0.455 \\
\hline unknown & -10.235 & -9.531 & 0.0416 & 0.3335 & -10.170 & -10.124 & 0.9187 & 0.9804 & -9.886 & -10.518 & 0.1850 & 0.45 \\
\hline unknown & -7.676 & -7.310 & 0.0333 & 0.2953 & -7.687 & -7.506 & 0.5861 & 0.9350 & -7.448 & -7.828 & 0.1633 & 0.436 \\
\hline unknown & -10.546 & -10.580 & 0.6844 & 0.8363 & -10.472 & -10.615 & 0.3396 & 0.9350 & -10.611 & -10.732 & 0.2200 & 0.493 \\
\hline unknown & -8.941 & -8.945 & 0.9620 & 0.9620 & -9.019 & -8.972 & 0.6779 & 0.9368 & -9.041 & -9.012 & 0.6324 & 0.809 \\
\hline unknown & -9.186 & -9.156 & 0.6572 & 0.8291 & -9.145 & -9.092 & 0.6314 & 0.9368 & -9.456 & -9.258 & 0.0226 & 0.393 \\
\hline unknown & -9.686 & -9.697 & 0.9050 & 0.9050 & -9.748 & -9.740 & 0.9603 & 0.9848 & -9.845 & -9.928 & 0.4719 & 0.700 \\
\hline unknown & -9.193 & -8.975 & 0.2604 & 0.5816 & -9.188 & -8.963 & 0.3694 & 0.9350 & -9.251 & -9.052 & 0.3185 & 0.576 \\
\hline unknown & -10.044 & -9.793 & 0.4993 & 0.7684 & -9.512 & -9.681 & 0.6958 & 0.9368 & -9.037 & -10.164 & 0.0825 & 0.393 \\
\hline unknown & -10.024 & -10.147 & 0.5528 & 0.7953 & -10.262 & -10.771 & 0.3627 & 0.9350 & -10.720 & -10.488 & 0.5134 & 0.731 \\
\hline unknown & -9.803 & -9.751 & 0.6078 & 0.8186 & -9.874 & -9.712 & 0.2391 & 0.9350 & -10.033 & -9.948 & 0.3640 & 0.613 \\
\hline unknown & -10.840 & -13.459 & 0.1241 & 0.4520 & -12.270 & -10.540 & 0.2968 & 0.9350 & -13.473 & -13.426 & 0.9422 & 0.966 \\
\hline unknown & -10.346 & -10.238 & 0.6330 & 0.8224 & -9.970 & -10.015 & 0.9013 & 0.9792 & -9.674 & -10.462 & 0.1133 & 0.411 \\
\hline unknown & -10.732 & -10.973 & 0.3276 & 0.6346 & -10.688 & -11.039 & 0.1832 & 0.9350 & -11.130 & -11.021 & 0.8217 & 0. \\
\hline
\end{tabular}




\begin{tabular}{|c|c|c|c|c|c|c|c|c|c|c|c|c|}
\hline unknown & -9.147 & -9.564 & 0.1583 & 0.4757 & -8.955 & -9.826 & 0.0335 & 0.9350 & -9.609 & -9.664 & 0.9224 & 0.9475 \\
\hline unknown & -9.505 & -9.567 & 0.6683 & 0.8363 & -9.580 & -9.500 & 0.4701 & 0.9350 & -9.659 & -9.533 & 0.3488 & 0.5993 \\
\hline unknown & -8.037 & -7.259 & 0.0223 & 0.2663 & -7.644 & -7.417 & 0.5974 & 0.9350 & -7.218 & -7.898 & 0.2027 & 0.4774 \\
\hline unknown & -10.074 & -10.062 & 0.9201 & 0.9201 & -10.098 & -9.986 & 0.4097 & 0.9350 & -10.170 & -10.091 & 0.2593 & 0.5276 \\
\hline unknown & -10.066 & -10.045 & 0.8579 & 0.8985 & -10.134 & -10.069 & 0.5576 & 0.9350 & -10.254 & -10.238 & 0.7266 & 0.8585 \\
\hline unknown & -10.116 & -10.186 & 0.4021 & 0.6782 & -10.160 & -10.150 & 0.9511 & 0.9848 & -10.447 & -10.346 & 0.4952 & 0.7195 \\
\hline unknown & -10.055 & -10.023 & 0.8095 & 0.8834 & -9.941 & -10.020 & 0.7134 & 0.9458 & -10.404 & -10.127 & 0.1513 & 0.4361 \\
\hline unknown & -10.386 & -10.410 & 0.8389 & 0.8868 & -10.381 & -10.291 & 0.4930 & 0.9350 & -10.989 & -10.537 & 0.0705 & 0.3934 \\
\hline unknown & -9.416 & -9.561 & 0.3290 & 0.6346 & -9.520 & -9.611 & 0.7152 & 0.9458 & -9.960 & -9.649 & 0.4369 & 0.6721 \\
\hline unknown & -10.941 & -10.806 & 0.5760 & 0.8025 & -11.104 & -10.731 & 0.4159 & 0.9350 & -10.841 & -11.633 & 0.0706 & 0.3934 \\
\hline unknown & -8.826 & -7.644 & 0.0334 & 0.2953 & -8.010 & -8.208 & 0.5202 & 0.9350 & -7.949 & -8.519 & 0.1825 & 0.4554 \\
\hline unknown & -11.247 & -10.950 & 0.3455 & 0.6476 & -11.356 & -11.044 & 0.4323 & 0.9350 & -10.285 & -11.259 & 0.2295 & 0.4977 \\
\hline unknown & -8.785 & -8.090 & 0.0965 & 0.4262 & -8.564 & -8.769 & 0.6075 & 0.9350 & -8.039 & -8.823 & 0.1440 & 0.4361 \\
\hline unknown & -9.974 & -9.626 & 0.1904 & 0.5092 & -9.905 & -10.001 & 0.6842 & 0.9368 & -10.018 & -10.059 & 0.5957 & 0.7870 \\
\hline unknown & -10.840 & -10.608 & 0.4902 & 0.7584 & -11.069 & -11.020 & 0.8929 & 0.9758 & -11.702 & -11.041 & 0.0768 & 0.3934 \\
\hline unknown & -6.120 & -6.160 & 0.7154 & 0.8502 & -6.336 & -6.158 & 0.1649 & 0.9350 & -6.322 & -6.261 & 0.3982 & 0.6472 \\
\hline unknown & -9.678 & -9.545 & 0.8440 & 0.8905 & -9.808 & -10.073 & 0.4835 & 0.9350 & -9.236 & -9.266 & 0.9500 & 0.9708 \\
\hline unknown & -10.791 & -10.948 & 0.7205 & 0.8518 & -10.656 & -11.517 & 0.0242 & 0.9350 & -11.511 & -11.487 & 0.9471 & 0.9696 \\
\hline unknown & -8.581 & -8.476 & 0.6894 & 0.8372 & -8.376 & -8.924 & 0.0914 & 0.9350 & -8.239 & -8.737 & 0.0367 & 0.3934 \\
\hline unknown & -7.620 & -7.469 & 0.3578 & 0.6476 & -7.584 & -7.411 & 0.0245 & 0.9350 & -7.609 & -7.555 & 0.5255 & 0.7413 \\
\hline unknown & -9.276 & -9.536 & 0.3041 & 0.6168 & -9.331 & -9.850 & 0.1853 & 0.9350 & -10.035 & -9.808 & 0.7024 & 0.8455 \\
\hline unknown & -9.380 & -9.539 & 0.5321 & 0.7826 & -9.315 & -9.853 & 0.1856 & 0.9350 & -9.753 & -9.848 & 0.6778 & 0.8358 \\
\hline unknown & -8.933 & -8.899 & 0.7285 & 0.8551 & -9.000 & -8.978 & 0.8623 & 0.9665 & -9.088 & -9.039 & 0.5785 & 0.7717 \\
\hline unknown & -8.169 & -7.992 & 0.5123 & 0.7776 & -7.526 & -7.783 & 0.5216 & 0.9350 & -6.973 & -7.900 & 0.0713 & 0.3934 \\
\hline unknown & -9.742 & -9.812 & 0.5061 & 0.7727 & -9.837 & -9.802 & 0.8246 & 0.9665 & -9.953 & -9.738 & 0.0432 & 0.3934 \\
\hline unknown & -8.913 & -8.981 & 0.4953 & 0.7643 & -8.910 & -9.046 & 0.4138 & 0.9350 & -9.356 & -9.161 & 0.4254 & 0.6594 \\
\hline unknown & -9.593 & -9.586 & 0.9564 & 0.9564 & -9.672 & -9.553 & 0.3259 & 0.9350 & -9.823 & -9.641 & 0.0245 & 0.3934 \\
\hline unknown & -9.654 & -9.681 & 0.7495 & 0.8597 & -9.684 & -9.681 & 0.9594 & 0.9848 & -9.779 & -9.799 & 0.8462 & 0.9194 \\
\hline unknown & -9.766 & -9.702 & 0.5513 & 0.7953 & -9.894 & -9.679 & 0.1666 & 0.9350 & -10.090 & -9.967 & 0.0773 & 0.3934 \\
\hline unknown & -9.765 & -9.699 & 0.6046 & 0.8169 & -9.648 & -9.748 & 0.4937 & 0.9350 & -10.124 & -9.862 & 0.2492 & 0.5187 \\
\hline unknown & -10.129 & -10.172 & 0.7393 & 0.8557 & -10.174 & -10.132 & 0.7742 & 0.9665 & -10.390 & -10.124 & 0.0376 & 0.3934 \\
\hline unknown & -10.375 & -9.763 & 0.0066 & 0.1751 & -10.560 & -10.559 & 0.9985 & 0.9985 & -10.052 & -10.629 & 0.1269 & 0.4215 \\
\hline unknown & -10.561 & -10.066 & 0.0341 & 0.2964 & -10.637 & -10.473 & 0.6198 & 0.9350 & -10.347 & -10.734 & 0.3125 & 0.5752 \\
\hline unknown & -8.472 & -8.152 & 0.2893 & 0.6073 & -8.166 & -7.765 & 0.4165 & 0.9350 & -7.853 & -8.637 & 0.1113 & 0.4114 \\
\hline unknown & -10.244 & -10.141 & 0.8078 & 0.8834 & -10.279 & -10.702 & 0.3541 & 0.9350 & -11.523 & -10.954 & 0.0455 & 0.3934 \\
\hline unknown & -11.387 & -10.748 & 0.1822 & 0.5054 & -11.115 & -10.793 & 0.6024 & 0.9350 & -10.473 & -11.789 & 0.0784 & 0.3934 \\
\hline unknown & -9.354 & -8.762 & 0.0595 & 0.3750 & -9.197 & -9.289 & 0.8477 & 0.9665 & -9.592 & -9.687 & 0.7100 & 0.8495 \\
\hline unknown & -10.608 & -10.066 & 0.0414 & 0.3335 & -10.869 & -11.173 & 0.4051 & 0.9350 & -11.672 & -11.712 & 0.8761 & 0.9333 \\
\hline unknown & -5.930 & -5.539 & 0.3399 & 0.6417 & -5.927 & -6.263 & 0.4228 & 0.9350 & -7.916 & -7.036 & 0.1020 & 0.4106 \\
\hline unknown & -9.178 & -8.363 & 0.1185 & 0.4495 & -8.818 & -9.435 & 0.3881 & 0.9350 & -11.208 & -9.265 & 0.1261 & 0.4215 \\
\hline unknown & -10.217 & -9.977 & 0.3477 & 0.6476 & -10.105 & -10.186 & 0.7510 & 0.9605 & -9.817 & -10.264 & 0.1376 & 0.4345 \\
\hline unknown & -9.089 & -8.610 & 0.0528 & 0.3484 & -8.995 & -9.144 & 0.7362 & 0.9547 & -9.494 & -9.790 & 0.1017 & 0.4106 \\
\hline unknown & -10.170 & -10.237 & 0.5423 & 0.7900 & -10.244 & -10.180 & 0.5221 & 0.9350 & -10.303 & -10.198 & 0.5058 & 0.7262 \\
\hline unknown & -10.344 & -10.363 & 0.8899 & 0.9024 & -10.442 & -10.398 & 0.7092 & 0.9458 & -10.280 & -10.431 & 0.4070 & 0.6510 \\
\hline unknown & -9.029 & -8.699 & 0.5246 & 0.7826 & -9.009 & -9.206 & 0.4849 & 0.9350 & -8.656 & -8.815 & 0.6600 & 0.8240 \\
\hline unknown & -6.083 & -5.373 & 0.0153 & 0.2344 & -6.357 & -5.827 & 0.3137 & 0.9350 & -7.218 & -6.095 & 0.0298 & 0.3934 \\
\hline unknown & -9.181 & -7.929 & 0.0219 & 0.2663 & -9.643 & -8.721 & 0.2783 & 0.9350 & -11.331 & -9.265 & 0.0384 & 0.3934 \\
\hline unknown & -9.866 & -9.246 & 0.4299 & 0.7050 & -9.529 & -10.066 & 0.0799 & 0.9350 & -9.528 & -9.870 & 0.3914 & 0.6413 \\
\hline unknown & -8.484 & -7.740 & 0.1504 & 0.4702 & -7.657 & -8.143 & 0.4032 & 0.9350 & -10.092 & -8.542 & 0.0982 & 0.4106 \\
\hline unknown & -7.681 & -7.947 & 0.1140 & 0.4451 & -7.908 & -7.777 & 0.5145 & 0.9350 & -7.915 & -7.838 & 0.6923 & 0.8411 \\
\hline unknown & -8.261 & -8.064 & 0.5510 & 0.7953 & -8.172 & -8.508 & 0.1391 & 0.9350 & -7.890 & -8.192 & 0.1626 & 0.4367 \\
\hline unknown & -9.384 & -9.266 & 0.7504 & 0.8597 & -8.995 & -9.262 & 0.3629 & 0.9350 & -10.102 & -9.338 & 0.0321 & 0.3934 \\
\hline unknown & -4.125 & -4.223 & 0.2623 & 0.5817 & -4.277 & -4.201 & 0.4145 & 0.9350 & -4.291 & -4.284 & 0.8757 & 0.9333 \\
\hline unknown & -7.430 & -7.541 & 0.6001 & 0.8163 & -7.441 & -7.263 & 0.2204 & 0.9350 & -7.418 & -7.294 & 0.1307 & 0.4215 \\
\hline unknown & -9.653 & -9.985 & 0.2018 & 0.5170 & -9.454 & -10.142 & 0.0326 & 0.9350 & -10.408 & -10.286 & 0.7089 & 0.8495 \\
\hline unknown & -8.131 & -8.465 & 0.5029 & 0.7699 & -8.619 & -9.469 & 0.1265 & 0.9350 & -8.930 & -8.723 & 0.7184 & 0.8519 \\
\hline unknown & -10.060 & -9.886 & 0.3826 & 0.6615 & -9.937 & -10.181 & 0.2587 & 0.9350 & -9.968 & -10.059 & 0.5687 & 0.7689 \\
\hline unknown & -9.153 & -9.124 & 0.8701 & 0.8985 & -8.976 & -8.941 & 0.8381 & 0.9665 & -9.098 & -9.065 & 0.8630 & 0.9268 \\
\hline unknown & -9.901 & -9.389 & 0.0930 & 0.4262 & -9.759 & -9.462 & 0.5023 & 0.9350 & -9.165 & -9.826 & 0.1014 & 0.4106 \\
\hline unknown & -10.595 & -10.183 & 0.3628 & 0.6487 & -10.565 & -10.624 & 0.8496 & 0.9665 & -9.109 & -10.084 & 0.4062 & 0.6510 \\
\hline unknown & -10.864 & -10.449 & 0.0766 & 0.4088 & -10.645 & -10.495 & 0.6606 & 0.9368 & -10.247 & -10.767 & 0.1209 & 0.4205 \\
\hline unknown & -8.644 & -8.842 & 0.3756 & 0.6533 & -8.722 & -9.157 & 0.1503 & 0.9350 & -9.534 & -9.086 & 0.5452 & 0.7524 \\
\hline unknown & -9.314 & -9.498 & 0.6441 & 0.8253 & -9.258 & -9.860 & 0.1370 & 0.9350 & -10.291 & -9.711 & 0.4652 & 0.6944 \\
\hline unknown & -7.675 & -7.281 & 0.0552 & 0.3603 & -7.492 & -7.427 & 0.8004 & 0.9665 & -7.063 & -7.634 & 0.1447 & 0.4361 \\
\hline unknown & -10.037 & -10.269 & 0.6859 & 0.8363 & -10.934 & -10.313 & 0.4013 & 0.9350 & -10.344 & -10.530 & 0.1988 & 0.4721 \\
\hline unknown & -11.523 & -10.432 & 0.1305 & 0.4521 & -10.348 & -10.560 & 0.5902 & 0.9350 & -10.051 & -11.041 & 0.2545 & 0.5238 \\
\hline unknown & -9.651 & -9.759 & 0.6863 & 0.8363 & -9.580 & -10.109 & 0.2101 & 0.9350 & -10.282 & -9.997 & 0.6492 & 0.8215 \\
\hline unknown & -9.209 & -8.849 & 0.0116 & 0.2113 & -9.298 & -8.945 & 0.1414 & 0.9350 & -8.992 & -9.080 & 0.7395 & 0.8659 \\
\hline unknown & -10.470 & -10.070 & 0.0518 & 0.3484 & -10.435 & -10.407 & 0.9376 & 0.9848 & -10.355 & -11.239 & 0.0644 & 0.3934 \\
\hline unknown & -10.557 & -9.902 & 0.3256 & 0.6346 & -9.994 & -10.390 & 0.5031 & 0.9350 & -9.421 & -10.673 & 0.1535 & 0.4366 \\
\hline unknown & -10.049 & -9.738 & 0.3206 & 0.6323 & -9.936 & -10.344 & 0.1943 & 0.9350 & -11.029 & -10.230 & 0.1443 & 0.4361 \\
\hline unknown & -10.568 & -10.341 & 0.6718 & 0.8363 & -10.601 & -10.685 & 0.7818 & 0.9665 & -9.809 & -9.987 & 0.7275 & 0.858 \\
\hline unknown & -9.794 & -8.829 & 0.0691 & 0.3888 & -9.388 & -9.567 & 0.6689 & 0.9368 & -8.934 & -9.712 & 0.2967 & 0.568 \\
\hline unknown & -9.906 & -9.694 & 0.7815 & 0.8762 & -10.065 & -10.606 & 0.4003 & 0.9350 & -9.524 & -9.495 & 0.9547 & 0.970 \\
\hline
\end{tabular}




\begin{tabular}{|c|c|c|c|c|c|c|c|c|c|c|c|c|}
\hline unknown & -12.079 & -11.152 & 0.3503 & 0.6476 & -11.376 & -12.604 & 0.2120 & 0.9350 & -12.570 & -12.385 & 0.6562 & 0.824 \\
\hline unknown & -9.192 & -9.015 & 0.7797 & 0.8762 & -9.050 & -9.766 & 0.0432 & 0.9350 & -10.343 & -10.254 & 0.8916 & 0.938 \\
\hline unknown & -9.983 & -9.453 & 0.0333 & 0.2953 & -10.520 & -10.396 & 0.6280 & 0.9350 & -9.863 & -10.141 & 0.1637 & 0.436 \\
\hline unknown & -8.216 & -8.485 & 0.2392 & 0.5582 & -8.288 & -8.839 & 0.2598 & 0.9350 & -9.538 & -8.817 & 0.5486 & 755 \\
\hline unknown & -11.509 & -11.367 & 0.6473 & 0.8253 & -11.361 & -11.571 & 0.5563 & 0.9350 & -10.080 & -11.019 & 0.0512 & 0.393 \\
\hline unknown & -8.920 & -8.855 & 0.3735 & 0.6533 & -8.957 & -8.849 & 0.2417 & 0.9350 & -9.124 & -8.932 & 0.0598 & 393 \\
\hline unknown & -10.295 & -9.865 & 0.1711 & 0.4907 & -9.891 & -9.827 & 0.8511 & 0.9665 & -9.748 & -10.049 & 0.2157 & \\
\hline unknown & -11.395 & -9.645 & 0.0100 & 0.1949 & -11.671 & -10.477 & 0.1287 & 0.9350 & -12.876 & -10.822 & 0.0288 & 393 \\
\hline unknown & -9.178 & -9.098 & 0.6499 & 0.8253 & -9.113 & -9.327 & 0.5048 & 0.9350 & -9.057 & -9.413 & 0.1903 & \\
\hline unknown & -10.364 & -10.015 & 0.0826 & 0.4121 & -10.007 & -10.086 & 0.8336 & 0.9665 & -9.752 & -10.104 & 0.3375 & 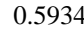 \\
\hline unknown & -5.686 & -5.167 & 0.0594 & 0.3750 & -5.496 & -5.435 & 0.8351 & 0.9665 & -4.933 & -5.749 & 0.1254 & 42 \\
\hline unknown & -8.627 & -8.078 & 0.1542 & 0.4709 & -8.252 & -8.277 & 0.9588 & 0.9848 & -7.975 & -8.698 & 0.1024 & 0.41 \\
\hline unknown & -10.051 & -10.073 & 0.8203 & 0.8834 & -10.107 & -9.881 & 0.1196 & 0.9350 & -10.104 & -10.089 & 0.8800 & \\
\hline unknown & -9.256 & -9.158 & 0.4355 & 0.7101 & -9.302 & -9.153 & 0.2460 & 0.9350 & -9.397 & -9.278 & 0.2495 & 0.518 \\
\hline unknown & -9.275 & -9.230 & 0.6424 & 0.8249 & -9.308 & -9.184 & 0.3384 & 0.9350 & -9.409 & -9.326 & 0.5533 & 0.75 \\
\hline unknown & -9.325 & -9.308 & 0.8230 & 0.8847 & -9.331 & -9.281 & 0.6685 & 0.9368 & -9.676 & -9.516 & 0.0145 & . \\
\hline unknown & -7.455 & -7.288 & 0.0909 & 0.4242 & -7.221 & -7.401 & 0.0170 & 0.9350 & -7.310 & -7.207 & 0.4975 & 72 \\
\hline unknown & -7.285 & -7.118 & 0.5977 & 0.8152 & -7.135 & -7.661 & 0.1824 & 0.9350 & -8.147 & -7.840 & 0.2826 & S \\
\hline unknown & -9.172 & -8.996 & 0.1131 & 0.4451 & -8.940 & -9.051 & 0.3253 & 0.9350 & -8.977 & -8.915 & 0.6796 & 836 \\
\hline unknown & -11.642 & -11.510 & 0.6570 & 0.8291 & -10.941 & -11.342 & 0.0883 & 0.9350 & -10.741 & -11.319 & 0.2538 & 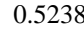 \\
\hline unknown & -8.088 & -7.936 & 0.6498 & 0.8253 & -7.951 & -8.483 & 0.1187 & 0.9350 & -9.455 & -9.080 & 0.3075 & 51 \\
\hline unknown & -9.852 & -9.572 & 0.4041 & 0.6782 & -9.639 & -10.196 & 0.1519 & 0.9350 & -10.883 & -10.406 & 0.2254 & \\
\hline unknown & -6.816 & -6.613 & 0.3826 & 0.6615 & -6.726 & -7.065 & 0.1803 & 0.9350 & -8.309 & -7.953 & 0.1526 & 436 \\
\hline unknown & -6.595 & -6.194 & 0.0959 & 0.4262 & -6.611 & -6.663 & 0.8785 & 0.9712 & -8.040 & -7.682 & 0.3088 & 575 \\
\hline unknown & -9.710 & -9.596 & 0.6420 & 0.8249 & -9.429 & -9.256 & 0.6649 & 0.9368 & -8.882 & -9.628 & 0.1037 & $0 \rightarrow 112$ \\
\hline unknown & -7.394 & -7.014 & 0.0835 & 0.4121 & -7.359 & -7.476 & 0.6965 & 0.9368 & -8.236 & -7.514 & 0.1608 & 0.43 \\
\hline unknown & -9.201 & -8.954 & 0.2717 & 0.5954 & -9.063 & -9.372 & 0.3133 & 0.9350 & -10.198 & -9.888 & 0.1717 & 0.445 \\
\hline unknown & -9.018 & -8.978 & 0.8516 & 0.8953 & -8.876 & -8.718 & 0.6973 & 0.9368 & -8.255 & -9.121 & 0.0741 & $39 ?$ \\
\hline unknown & -15.867 & -13.238 & 0.1979 & 0.5141 & -11.106 & -14.539 & 0.1379 & 0.9350 & -16.079 & -14.288 & 0.1633 & 436 \\
\hline unknown & -10.212 & -10.284 & 0.6903 & 0.8372 & -10.036 & -10.186 & 0.5124 & 0.9350 & -10.032 & -10.221 & 0.4948 & $71 \mathrm{c}$ \\
\hline unknown & -8.621 & -8.947 & 0.3965 & 0.6753 & -8.448 & -9.221 & 0.0302 & 0.9350 & -9.500 & -9.302 & 0.7744 & \\
\hline unknown & -10.895 & -10.906 & 0.9888 & 0.9888 & -10.669 & -12.004 & 0.0251 & 0.9350 & -12.008 & -11.782 & 0.7842 & 390 \\
\hline unknown & -5.387 & -5.340 & 0.6851 & 0.8363 & -5.359 & -5.430 & 0.4856 & 0.9350 & -5.391 & -5.363 & 0.8614 & 926 \\
\hline unknown & -9.919 & -9.303 & 0.0449 & 0.3391 & -9.460 & -9.085 & 0.3697 & 0.9350 & -8.952 & -9.528 & 0.1842 & \\
\hline unknown & -8.687 & -8.309 & 0.1328 & 0.4537 & -8.388 & -8.490 & 0.7819 & 0.9665 & -7.955 & -8.804 & 0.0575 & 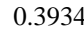 \\
\hline unknown & -6.122 & -6.044 & 0.2692 & 0.5924 & -6.140 & -6.047 & 0.2540 & 0.9350 & -6.208 & -6.126 & 0.4936 & 11 \\
\hline unknown & -7.681 & -7.635 & 0.6728 & 0.8363 & -7.749 & -7.602 & 0.1922 & 0.9350 & -7.673 & -7.706 & 0.7763 & 88 \\
\hline unknown & -9.143 & -8.883 & 0.1286 & 0.4521 & -9.152 & -9.088 & 0.6343 & 0.9368 & -8.989 & -9.072 & 0.6189 & 0.80 \\
\hline unknown & -8.396 & -8.360 & 0.7348 & 0.8557 & -8.408 & -8.523 & 0.4893 & 0.9350 & -8.558 & -8.554 & 0.9785 & 0.98 \\
\hline unknown & -8.386 & -8.360 & 0.7993 & 0.8834 & -8.406 & -8.523 & 0.4813 & 0.9350 & -8.558 & -8.525 & 0.8282 & 0.910 \\
\hline unknown & -6.898 & -7.150 & 0.0583 & 0.3750 & -6.944 & -7.257 & 0.1824 & 0.9350 & -7.483 & -7.402 & 0.8144 & \\
\hline unknown & -9.695 & -9.638 & 0.4782 & 0.7488 & -9.721 & -9.604 & 0.4458 & 0.9350 & -9.165 & -9.674 & 0.3681 & 0.614 \\
\hline unknown & -9.258 & -9.221 & 0.7724 & 0.8745 & -9.335 & -9.252 & 0.3322 & 0.9350 & -9.502 & -9.383 & 0.4136 & 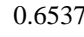 \\
\hline unknown & -10.629 & -9.626 & 0.0074 & 0.1837 & -10.173 & -9.986 & 0.6705 & 0.9368 & -9.778 & -9.884 & 0.6430 & 0.8162 \\
\hline unknown & -7.585 & -7.175 & 0.0434 & 0.3361 & -7.361 & -7.168 & 0.5080 & 0.9350 & -7. & -7.381 & 0.3031 & 1. \\
\hline unknown & -7.806 & -7.298 & 0.0479 & 0.3484 & -7.709 & -7.425 & 0.4092 & 0.9350 & -7.478 & -7.845 & 0.3952 & 64 \\
\hline unknown & -10.104 & -9.984 & 0.2843 & 0.6066 & -10.141 & -10.192 & 0.7627 & 0.9658 & -9.981 & -10.156 & 0.6585 & 82 \\
\hline unknown & -9.846 & -9.614 & 0.0758 & 0.4088 & -9.851 & -9.736 & 0.4294 & 0.9350 & -9.425 & -9.808 & 0.3820 & 0.63 \\
\hline unknown & -8.190 & -8.189 & 0.9925 & 0.9925 & -8.149 & -8.143 & 0.9559 & 0.9848 & -8.168 & -8.219 & 0.6881 & 0.841 \\
\hline unknown & -9.180 & -9.092 & 0.1873 & 0.5071 & -9.192 & -9.160 & 0.8337 & 0.9665 & -9.245 & -9.263 & 0.6316 & \\
\hline unknown & -9.815 & -9.776 & 0.5408 & 0.7899 & -9.955 & -9.749 & 0.2556 & 0.9350 & -9.895 & -9.772 & 0.3111 & 0.5752 \\
\hline unknown & -8.810 & -8.755 & 0.7260 & 0.8539 & -8.891 & -8.822 & 0.5621 & 0.9350 & -8.804 & -8.887 & 0.6523 & 0.0210 \\
\hline unknown & -8.779 & -8.765 & 0.8734 & 0.8985 & -8.699 & -8.741 & 0.5948 & 0.9350 & -8.979 & -8.849 & 0.1102 & 0.4114 \\
\hline unknown & -10.484 & -9.968 & 0.1050 & 0.4366 & -10.086 & -9.911 & 0.5821 & 0.9350 & -9.710 & -10.134 & 0.2226 & 0 \\
\hline unknown & -10.060 & -9.316 & 0.0249 & 0.2856 & -9.927 & -9.526 & 0.3526 & 0.9350 & -9.886 & -9.493 & 0.1065 & \\
\hline unknown & -9.937 & -9.949 & 0.8973 & 0.9024 & -9.780 & -9.850 & 0.4130 & 0.9350 & -10.091 & -10.011 & 0.4186 & \\
\hline unknown & -10.980 & -10.685 & 0.3302 & 0.6346 & -11.287 & -11.190 & 0.3791 & 0.9350 & -10.488 & -11.198 & 0.0943 & 02 \\
\hline unknown & -10.297 & -9.545 & 0.0269 & 0.2953 & -10.294 & -10.204 & 0.8439 & 0.9665 & -9.860 & -10.365 & 0.0960 & 40 \\
\hline unknown & -9.992 & -9.952 & 0.8306 & 0.8861 & -10.013 & -10.164 & 0.1675 & 0.9350 & -10.566 & -10.028 & 0.0043 & 0303 \\
\hline unknown & -10.012 & -9.125 & 0.0025 & 0.1459 & -10.502 & -10.635 & 0.7519 & 0.9605 & -10.439 & -10.628 & 0.3232 & 0.580 \\
\hline unknown & -7.491 & -7.966 & 0.1526 & 0.4709 & -7.424 & -8.207 & 0.0423 & 0.9350 & -8.147 & -8.304 & 0.7594 & 0.879 \\
\hline unknown & -9.566 & -9.124 & 0.1362 & 0.4557 & -9.066 & -9.069 & 0.9942 & 0.9985 & -8.312 & -9.403 & 0.0463 & . \\
\hline unknown & -8.275 & -8.737 & 0.1308 & 0.4521 & -8.182 & -9.049 & 0.0349 & 0.9350 & -9.330 & -9.505 & 0.7583 & 0.7 \\
\hline unknown & -7.987 & -8.235 & 0.1868 & 0.5071 & -7.918 & -8.066 & 0.5560 & 0.9350 & -7.712 & -8.067 & 0.1729 & \\
\hline unknown & -10.098 & -9.812 & 0.3651 & 0.6488 & -9.959 & -10.054 & 0.6279 & 0.9350 & -9.736 & -9.870 & 0.6661 & 00 \\
\hline unknown & -11.079 & -9.920 & 0.0173 & 0.2423 & -11.439 & -10.423 & 0.1947 & 0.9350 & -12.340 & -10.967 & 0.1175 & \\
\hline unknown & -9.953 & -10.176 & 0.3126 & 0.6255 & -10.027 & -10.472 & 0.3749 & 0.9350 & -10.669 & -10.910 & 0.5965 & \\
\hline unknown & -10.436 & -10.148 & 0.5485 & 0.7953 & -10.142 & -10.020 & 0.7561 & 0.9619 & -9.500 & -10.159 & 0.1984 & 17 \\
\hline unknown & -6.806 & -6.522 & 0.2694 & 0.5924 & -6.586 & -6.870 & 0.2424 & 0.9350 & -6.443 & -6.763 & 0.1604 & 0.450 \\
\hline unknown & -11.161 & -10.430 & 0.0220 & 0.2663 & -11.091 & -10.584 & 0.3210 & 0.9350 & -10.719 & -11.343 & 0.2315 & 0.500 \\
\hline unknown & -10.928 & -10.611 & 0.1538 & 0.4709 & -10.594 & -10.604 & 0.9675 & 0.9876 & -10.427 & -10.925 & 0.1432 & 0.436 \\
\hline unknown & -8.161 & -7.720 & 0.1636 & 0.4792 & -8.066 & -8.031 & 0.9398 & 0.9848 & -7.969 & -8.364 & 0.2850 & \\
\hline unknown & -9.576 & -9.132 & 0.0876 & 0.4175 & -9.962 & -9.854 & 0.8588 & 0.9665 & -10.402 & -9.968 & 0.3292 & \\
\hline unknown & -10.200 & -10.035 & 0.2834 & 0.6066 & -10.081 & -9.818 & 0.5406 & 0.9350 & -9.993 & -10.703 & 0.0500 & \\
\hline
\end{tabular}




\begin{tabular}{|c|c|c|c|c|c|c|c|c|c|c|c|c|}
\hline unknown & -10.765 & -11.299 & 0.2174 & 0.5380 & -10.456 & -11.553 & 0.0331 & 0.9350 & -11.836 & -11.839 & 0.9967 & 0.9967 \\
\hline unknown & -10.932 & -10.656 & 0.1222 & 0.4520 & -10.731 & -10.727 & 0.9904 & 0.9985 & -10.559 & -10.972 & 0.1553 & 0.4367 \\
\hline unknown & -8.750 & -8.482 & 0.2129 & 0.5306 & -8.766 & -8.930 & 0.4761 & 0.9350 & -8.802 & -8.836 & 0.8534 & 0.9229 \\
\hline unknown & -7.146 & -7.489 & 0.3516 & 0.6476 & -7.011 & -7.890 & 0.0271 & 0.9350 & -8.368 & -8.583 & 0.7091 & 0.8495 \\
\hline unknown & -9.341 & -8.690 & 0.0362 & 0.3099 & -8.980 & -8.794 & 0.6225 & 0.9350 & -8.630 & -9.357 & 0.1111 & 0.4114 \\
\hline unknown & -7.349 & -7.215 & 0.4428 & 0.7141 & -7.188 & -7.760 & 0.0712 & 0.9350 & -7.071 & -7.510 & 0.0549 & 0.3934 \\
\hline unknown & -8.632 & -9.058 & 0.2279 & 0.5509 & -8.477 & -9.298 & 0.0536 & 0.9350 & -9.049 & -9.308 & 0.5749 & 0.7717 \\
\hline unknown & -9.464 & -9.857 & 0.1206 & 0.4495 & -9.525 & -10.128 & 0.3464 & 0.9350 & -10.051 & -10.191 & 0.8005 & 0.8989 \\
\hline unknown & -10.333 & -10.429 & 0.6136 & 0.8186 & -10.209 & -10.583 & 0.3163 & 0.9350 & -10.786 & -10.854 & 0.7738 & 0.8865 \\
\hline unknown & -10.090 & -10.051 & 0.8320 & 0.8861 & -10.205 & -10.172 & 0.7817 & 0.9665 & -10.153 & -10.088 & 0.7742 & 0.8865 \\
\hline unknown & -10.422 & -10.274 & 0.6305 & 0.8224 & -10.514 & -10.879 & 0.1860 & 0.9350 & -9.651 & -10.553 & 0.1817 & 0.4554 \\
\hline unknown & -10.049 & -9.995 & 0.8659 & 0.8985 & -9.640 & -9.849 & 0.1869 & 0.9350 & -8.440 & -9.836 & 0.1456 & 0.4361 \\
\hline unknown & -10.656 & -9.961 & 0.0731 & 0.4037 & -10.414 & -10.668 & 0.5009 & 0.9350 & -9.995 & -10.665 & 0.1724 & 0.4455 \\
\hline unknown & -9.560 & -9.016 & 0.0935 & 0.4262 & -9.686 & -9.483 & 0.6415 & 0.9368 & -9.815 & -10.092 & 0.3458 & 0.5975 \\
\hline unknown & -10.213 & -9.529 & 0.0512 & 0.3484 & -10.177 & -10.008 & 0.7149 & 0.9458 & -9.738 & -10.456 & 0.1848 & 0.4554 \\
\hline unknown & -8.934 & -8.807 & 0.3283 & 0.6346 & -8.903 & -8.733 & 0.1817 & 0.9350 & -9.069 & -8.853 & 0.0640 & 0.3934 \\
\hline unknown & -8.045 & -7.432 & 0.0274 & 0.2953 & -8.029 & -7.978 & 0.9153 & 0.9804 & -8.454 & -8.507 & 0.8653 & 0.9268 \\
\hline unknown & -9.351 & -8.606 & 0.0155 & 0.2344 & -9.786 & -9.872 & 0.8539 & 0.9665 & -10.071 & -10.013 & 0.7753 & 0.8865 \\
\hline unknown & -10.567 & -10.112 & 0.1013 & 0.4313 & -10.336 & -10.238 & 0.8175 & 0.9665 & -10.413 & -11.065 & 0.0185 & 0.3934 \\
\hline unknown & -11.053 & -10.572 & 0.4123 & 0.6900 & -10.705 & -11.025 & 0.3215 & 0.9350 & -10.450 & -10.603 & 0.7325 & 0.8610 \\
\hline unknown & -8.490 & -7.993 & 0.0169 & 0.2423 & -8.358 & -8.342 & 0.9721 & 0.9885 & -8.336 & -8.707 & 0.0522 & 0.3934 \\
\hline unknown & -7.567 & -7.282 & 0.6136 & 0.8186 & -7.638 & -8.011 & 0.3128 & 0.9350 & -7.303 & -7.405 & 0.7835 & 0.8905 \\
\hline unknown & -11.838 & -11.520 & 0.6813 & 0.8363 & -12.115 & -11.980 & 0.5203 & 0.9350 & -11.238 & -11.529 & 0.4083 & 0.6513 \\
\hline unknown & -10.754 & -10.336 & 0.1479 & 0.4668 & -10.929 & -10.788 & 0.7780 & 0.9665 & -10.291 & -10.993 & 0.1220 & 0.4205 \\
\hline unknown & -8.374 & -8.370 & 0.9683 & 0.9683 & -8.408 & -8.381 & 0.8101 & 0.9665 & -8.363 & -8.421 & 0.6422 & 0.8162 \\
\hline unknown & -9.448 & -9.127 & 0.0622 & 0.3790 & -9.456 & -9.421 & 0.7657 & 0.9659 & -9.429 & -9.386 & 0.6593 & 0.8240 \\
\hline unknown & -6.502 & -6.394 & 0.6988 & 0.8374 & -6.485 & -6.763 & 0.2324 & 0.9350 & -6.318 & -6.523 & 0.2608 & 0.5276 \\
\hline unknown & -10.091 & -9.947 & 0.7988 & 0.8834 & -10.076 & -10.619 & 0.1075 & 0.9350 & -9.757 & -10.157 & 0.1297 & 0.4215 \\
\hline unknown & -11.141 & -10.747 & 0.1822 & 0.5054 & -10.785 & -10.198 & 0.2636 & 0.9350 & -10.535 & -11.245 & 0.1519 & 0.4361 \\
\hline unknown & -9.528 & -8.895 & 0.1115 & 0.4448 & -10.073 & -9.902 & 0.5560 & 0.9350 & -10.300 & -9.988 & 0.2968 & 0.5681 \\
\hline unknown & -10.295 & -9.821 & 0.0320 & 0.2953 & -10.369 & -10.304 & 0.7252 & 0.9486 & -10.451 & -10.396 & 0.6911 & 0.8411 \\
\hline unknown & -9.911 & -9.790 & 0.3328 & 0.6346 & -9.914 & -9.912 & 0.9909 & 0.9985 & -9.957 & -9.900 & 0.5546 & 0.7588 \\
\hline unknown & -8.883 & -8.894 & 0.9181 & 0.9181 & -8.872 & -8.854 & 0.8384 & 0.9665 & -9.011 & -8.926 & 0.3155 & 0.5752 \\
\hline unknown & -9.655 & -9.675 & 0.8660 & 0.8985 & -9.745 & -9.716 & 0.8110 & 0.9665 & -9.715 & -9.782 & 0.5930 & 0.7870 \\
\hline unknown & -10.431 & -9.915 & 0.0951 & 0.4262 & -10.916 & -10.854 & 0.8129 & 0.9665 & -11.607 & -11.041 & 0.1523 & 0.4361 \\
\hline unknown & -6.505 & -6.600 & 0.4284 & 0.7050 & -6.709 & -6.551 & 0.1136 & 0.9350 & -6.933 & -6.609 & 0.0073 & 0.3934 \\
\hline unknown & -6.374 & -6.386 & 0.8989 & 0.9024 & -6.513 & -6.381 & 0.2451 & 0.9350 & -6.586 & -6.403 & 0.1157 & 0.4119 \\
\hline unknown & -10.049 & -10.108 & 0.7446 & 0.8582 & -10.104 & -10.121 & 0.8880 & 0.9754 & -10.333 & -9.920 & 0.0657 & 0.3934 \\
\hline unknown & -10.860 & -10.275 & 0.0329 & 0.2953 & -11.273 & -11.426 & 0.7608 & 0.9658 & -10.760 & -11.300 & 0.1506 & 0.4361 \\
\hline unknown & -9.743 & -9.379 & 0.2313 & 0.5509 & -9.841 & -10.077 & 0.5577 & 0.9350 & -9.897 & -10.002 & 0.6676 & 0.8284 \\
\hline unknown & -11.191 & -10.002 & 0.0198 & 0.2588 & -10.202 & -9.961 & 0.7150 & 0.9458 & -9.500 & -10.461 & 0.1302 & 0.4215 \\
\hline unknown & -9.936 & -9.504 & 0.0493 & 0.3484 & -9.766 & -9.687 & 0.7684 & 0.9665 & -9.333 & -9.827 & 0.1306 & 0.4215 \\
\hline unknown & -8.442 & -7.949 & 0.0880 & 0.4175 & -8.301 & -8.614 & 0.3741 & 0.9350 & -8.021 & -8.827 & 0.0324 & 0.3934 \\
\hline unknown & -10.579 & -10.483 & 0.8201 & 0.8834 & -10.410 & -11.071 & 0.1219 & 0.9350 & -10.359 & -10.810 & 0.0442 & 0.3934 \\
\hline unknown & -9.542 & -9.469 & 0.5771 & 0.8025 & -9.610 & -9.510 & 0.3885 & 0.9350 & -9.533 & -9.649 & 0.5283 & 0.7413 \\
\hline unknown & -14.312 & -12.929 & 0.1170 & 0.4495 & -12.413 & -13.717 & 0.0759 & 0.9350 & -13.696 & -14.036 & 0.6050 & 0.7930 \\
\hline unknown & -9.462 & -9.448 & 0.8906 & 0.9024 & -9.495 & -9.370 & 0.2499 & 0.9350 & -9.605 & -9.484 & 0.2678 & 0.5330 \\
\hline unknown & -9.696 & -9.567 & 0.1148 & 0.4451 & -9.707 & -9.624 & 0.4715 & 0.9350 & -9.985 & -9.763 & 0.0362 & 0.3934 \\
\hline unknown & -11.090 & -10.073 & 0.0300 & 0.2953 & -10.740 & -10.877 & 0.6142 & 0.9350 & -10.295 & -10.984 & 0.2876 & 0.5587 \\
\hline unknown & -9.874 & -9.837 & 0.7900 & 0.8822 & -9.894 & -9.876 & 0.9000 & 0.9792 & -9.870 & -9.997 & 0.3568 & 0.6078 \\
\hline unknown & -10.056 & -10.095 & 0.6092 & 0.8186 & -10.166 & -10.147 & 0.8720 & 0.9665 & -10.491 & -10.265 & 0.0017 & 0.3934 \\
\hline unknown & -10.282 & -9.960 & 0.2305 & 0.5509 & -10.251 & -10.188 & 0.7012 & 0.9375 & -10.657 & -10.509 & 0.0766 & 0.3934 \\
\hline unknown & -10.367 & -10.302 & 0.7394 & 0.8557 & -10.365 & -10.441 & 0.7265 & 0.9486 & -10.887 & -10.646 & 0.3858 & 0.6356 \\
\hline unknown & -11.210 & -11.281 & 0.9079 & 0.9079 & -11.600 & -11.981 & 0.5123 & 0.9350 & -11.009 & -11.023 & 0.9778 & 0.9850 \\
\hline unknown & -9.960 & -9.250 & 0.1020 & 0.4313 & -10.118 & -9.895 & 0.6669 & 0.9368 & -10.061 & -10.222 & 0.5550 & 0.7588 \\
\hline unknown & -10.132 & -9.877 & 0.3128 & 0.6255 & -9.899 & -10.054 & 0.5665 & 0.9350 & -9.238 & -9.749 & 0.0696 & 0.3934 \\
\hline unknown & -10.020 & -10.071 & 0.6400 & 0.8249 & -10.064 & -9.953 & 0.2019 & 0.9350 & -10.287 & -10.039 & 0.0619 & 0.3934 \\
\hline unknown & -11.795 & -11.284 & 0.6216 & 0.8186 & -11.470 & -13.439 & 0.1326 & 0.9350 & -11.046 & -11.094 & 0.9042 & 0.9437 \\
\hline unknown & -10.182 & -10.152 & 0.5528 & 0.7953 & -10.338 & -10.192 & 0.3573 & 0.9350 & -10.419 & -10.161 & 0.0657 & 0.3934 \\
\hline unknown & -8.322 & -7.868 & 0.0627 & 0.3790 & -8.301 & -8.225 & 0.8226 & 0.9665 & -7.758 & -8.350 & 0.1592 & 0.4367 \\
\hline unknown & -6.923 & -6.991 & 0.3916 & 0.6710 & -7.066 & -6.901 & 0.1698 & 0.9350 & -7.044 & -6.922 & 0.2675 & 0.5330 \\
\hline unknown & -10.569 & -10.176 & 0.1258 & 0.4521 & -11.093 & -10.721 & 0.4089 & 0.9350 & -12.824 & -11.342 & 0.0622 & 0.3934 \\
\hline unknown & -9.933 & -9.356 & 0.0506 & 0.3484 & -10.467 & -9.662 & 0.1765 & 0.9350 & -12.075 & -10.305 & 0.0436 & 0.3934 \\
\hline unknown & -9.634 & -8.929 & 0.0130 & 0.2235 & -10.169 & -9.503 & 0.2552 & 0.9350 & -11.984 & -9.771 & 0.0452 & 0.3934 \\
\hline unknown & -9.941 & -9.290 & 0.1190 & 0.4495 & -10.784 & -9.868 & 0.3000 & 0.9350 & -12.552 & -10.072 & 0.0352 & 0.3934 \\
\hline unknown & -10.607 & -10.703 & 0.5550 & 0.7959 & -10.462 & -10.304 & 0.5898 & 0.9350 & -10.050 & -10.879 & 0.0718 & 0.3934 \\
\hline unknown & -10.470 & -9.789 & 0.0289 & 0.2953 & -10.870 & -10.328 & 0.3384 & 0.9350 & -12.747 & -10.605 & 0.0507 & 0.3934 \\
\hline unknown & -10.666 & -10.362 & 0.2962 & 0.6073 & -10.428 & -10.448 & 0.9508 & 0.9848 & -10.041 & -10.768 & 0.0467 & 0.3934 \\
\hline unknown & -8.063 & -8.043 & 0.8280 & 0.8855 & -8.145 & -8.037 & 0.3227 & 0.9350 & -8.192 & -8.071 & 0.4762 & 0.7055 \\
\hline unknown & -10.550 & -10.267 & 0.0673 & 0.3863 & -10.565 & -10.634 & 0.7449 & 0.9598 & -10.282 & -10.350 & 0.7131 & 0.8499 \\
\hline unknown & -10.044 & -9.626 & 0.1628 & 0.4792 & -10.034 & -10.218 & 0.6737 & 0.9368 & -10.316 & -9.585 & 0.2090 & 0.4828 \\
\hline unknown & -9.540 & -9.095 & 0.0433 & 0.3361 & -9.279 & -9.093 & 0.5338 & 0.9350 & -8.965 & -9.362 & 0.2162 & 0.4900 \\
\hline unknown & -10.397 & -9.764 & 0.0139 & 0.2235 & -10.347 & -10.340 & 0.9848 & 0.9980 & -10.171 & -10.357 & 0.4129 & 0.6537 \\
\hline unknown & -10.544 & -10.140 & 0.1203 & 0.4495 & -10.824 & -10.632 & 0.6968 & 0.9368 & -12.254 & -10.936 & 0.0743 & 0.3934 \\
\hline
\end{tabular}




\begin{tabular}{|c|c|c|c|c|c|c|c|c|c|c|c|c|}
\hline unknown & -10.130 & -9.655 & 0.1025 & 0.4313 & -10.364 & -10.067 & 0.5727 & 0.9350 & -11.638 & -10.113 & 0.0650 & 0.393 \\
\hline unknown & -10.292 & -9.521 & 0.0011 & 0.0907 & -10.420 & -10.337 & 0.7949 & 0.9665 & -10.328 & -10.529 & 0.3157 & 0.5752 \\
\hline unknown & -9.725 & -9.969 & 0.1848 & 0.5057 & -9.714 & -9.778 & 0.7914 & 0.9665 & -9.635 & -9.862 & 0.4144 & 0.6537 \\
\hline unknown & -8.926 & -8.655 & 0.3527 & 0.6476 & -8.742 & -8.757 & 0.9641 & 0.9871 & -8.468 & -8.754 & 0.3455 & 597 \\
\hline unknown & -7.887 & -7.663 & 0.3680 & 0.6520 & -7.689 & -7.975 & 0.2638 & 0.9350 & -7.496 & -7.916 & 0.0595 & \\
\hline unknown & -7.435 & -7.107 & 0.0635 & 0.3797 & -7.512 & -7.380 & 0.6886 & 0.9368 & -7.309 & -7.625 & 0.2530 & 523 \\
\hline unknown & -10.987 & -10.364 & 0.0232 & 0.2715 & -11.254 & -11.171 & 0.8702 & 0.9665 & -12.707 & -11.127 & 0.0270 & \\
\hline unknown & -8.711 & -8.458 & 0.2046 & 0.5219 & -9.497 & -8.695 & 0.2132 & 0.9350 & -9.626 & -8.890 & 0.0298 & 393 \\
\hline unknown & -8.282 & -8.045 & 0.0311 & 0.2953 & -8.383 & -8.559 & 0.6414 & 0.9368 & -9.259 & -8.564 & 0.0871 & 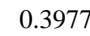 \\
\hline unknown & -9.620 & -9.463 & 0.3341 & 0.6350 & -9.860 & -9.640 & 0.3251 & 0.9350 & -9.822 & -9.676 & 0.4229 & .65 \\
\hline unknown & -8.220 & -7.914 & 0.0850 & 0.4121 & -8.401 & -8.305 & 0.7879 & 0.9665 & -9.407 & -8.575 & 0.0722 & 53 \\
\hline unknown & -9.047 & -9.046 & 0.9924 & 0.9924 & -9.220 & -9.066 & 0.5269 & 0.9350 & -9.810 & -9.265 & 0.0163 & 99. \\
\hline unknown & -10.052 & -9.856 & 0.0627 & 0.3790 & -10.227 & -10.107 & 0.6725 & 0.9368 & -10.566 & -10.157 & 0.0749 & 0.39 \\
\hline unknown & -8.579 & -7.995 & 0.1205 & 0.4495 & -8.537 & -8.367 & 0.7472 & 0.9605 & -9.588 & -8.124 & 0.0219 & 39. \\
\hline unknown & -8.014 & -7.158 & 0.0083 & 0.1837 & -8.157 & -7.625 & 0.3325 & 0.9350 & -9.319 & -7.682 & 0.0256 & 393 \\
\hline unknown & -8.761 & -8.295 & 0.2301 & 0.5509 & -9.073 & -8.313 & 0.1731 & 0.9350 & -10.566 & -8.597 & 0.0112 & 39. \\
\hline unknown & -9.893 & -9.372 & 0.0656 & 0.3863 & -9.757 & -9.933 & 0.5510 & 0.9350 & -9.681 & -9.928 & 0.0897 & $40 c$ \\
\hline unknown & -11.054 & -10.436 & 0.0481 & 0.3484 & -10.779 & -10.514 & 0.5226 & 0.9350 & -10.352 & -11.081 & 0.0835 & 59. \\
\hline unknown & -8.059 & -7.885 & 0.2946 & 0.6073 & -8.004 & -8.311 & 0.1372 & 0.9350 & -7.838 & -8.252 & 0.0652 & \\
\hline unknown & -8.690 & -9.005 & 0.0853 & 0.4121 & -9.074 & -8.834 & 0.4016 & 0.9350 & -9.419 & -8.954 & 0.1615 & 43 \\
\hline unknown & -9.946 & -10.023 & 0.5788 & 0.8025 & -10.080 & -10.046 & 0.8148 & 0.9665 & -9.907 & -10.005 & 0.6649 & 828 \\
\hline unknown & -9.998 & -10.146 & 0.1508 & 0.4702 & -10.040 & -10.097 & 0.7174 & 0.9466 & -10.024 & -10.127 & 0.4629 & 0.69 \\
\hline unknown & -8.793 & -8.689 & 0.4477 & 0.7179 & -8.804 & -9.076 & 0.2911 & 0.9350 & -8.606 & -8.952 & 0.0836 & .39 \\
\hline unknown & -10.920 & -10.336 & 0.0085 & 0.1837 & -10.748 & -10.710 & 0.9208 & 0.9804 & -10.879 & -10.824 & 0.8347 & 915 \\
\hline unknown & -10.938 & -10.346 & 0.1029 & 0.4313 & -10.768 & -10.937 & 0.6256 & 0.9350 & -10.390 & -10.922 & 0.2748 & 54 \\
\hline unknown & -9.651 & -8.879 & 0.0327 & 0.2953 & -9.705 & -9.565 & 0.8301 & 0.9665 & -11.260 & -9.278 & 0.0567 & 0.393 \\
\hline unknown & -10.229 & -10.311 & 0.6347 & 0.8224 & -10.439 & -10.271 & 0.1489 & 0.9350 & -10.680 & -10.235 & 0.0858 & 0.39 \\
\hline unknown & -9.185 & -9.003 & 0.2300 & 0.5509 & -9.260 & -9.031 & 0.1094 & 0.9350 & -9.233 & -9.145 & 0.2920 & 50 \\
\hline unknown & -10.005 & -9.520 & 0.0685 & 0.3888 & -10.141 & -10.047 & 0.8579 & 0.9665 & -10.468 & -10.647 & 0.4789 & \\
\hline unknown & -9.548 & -9.099 & 0.0086 & 0.1837 & -9.487 & -9.560 & 0.8719 & 0.9665 & -10.047 & -10.326 & 0.2720 & לכנס \\
\hline unknown & -10.294 & -9.775 & 0.0316 & 0.2953 & -10.165 & -10.209 & 0.9075 & 0.9804 & -10.073 & -10.432 & 0.2593 & 527 \\
\hline unknown & -8.512 & -8.330 & 0.6851 & 0.8363 & -8.642 & -8.890 & 0.3174 & 0.9350 & -8.356 & -8.454 & 0.7892 & \\
\hline unknown & -7.760 & -7.669 & 0.8056 & 0.8834 & -7.694 & -8.076 & 0.1648 & 0.9350 & -7.450 & -7.736 & 0.2418 & 51 \\
\hline unknown & -8.491 & -8.402 & 0.8066 & 0.8834 & -8.399 & -8.904 & 0.1256 & 0.9350 & -8.257 & -8.500 & 0.3253 & 582 \\
\hline unknown & -10.180 & -9.989 & 0.4279 & 0.7050 & -10.381 & -10.698 & 0.5124 & 0.9350 & -11.982 & -10.744 & 0.1028 & 0.41 \\
\hline unknown & -8.925 & -8.421 & 0.0849 & 0.4121 & -9.275 & -8.903 & 0.4497 & 0.9350 & -10.898 & -9.623 & 0.0525 & 393 \\
\hline unknown & -8.177 & -8.226 & 0.7215 & 0.8518 & -8.379 & -8.210 & 0.1542 & 0.9350 & -8.593 & -8.216 & 0.0587 & 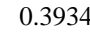 \\
\hline unknown & -6.947 & -6.993 & 0.5979 & 0.8152 & -7.128 & -7.000 & 0.2778 & 0.9350 & -7.221 & -7.011 & 0.0917 & \\
\hline unknown & -9.697 & -9.019 & 0.0063 & 0.1751 & -9.258 & -9.048 & 0.5879 & 0.9350 & -8.954 & -9.266 & 0.3850 & \\
\hline unknown & -10.735 & -10.462 & 0.3882 & 0.6672 & -10.744 & -10.980 & 0.5253 & 0.9350 & -11.165 & -10.997 & 0.3498 & 599 \\
\hline unknown & -10.417 & -10.135 & 0.1944 & 0.5120 & -10.228 & -10.301 & 0.6771 & 0.9368 & -10.037 & -10.243 & 0.4809 & \\
\hline unknown & -10.841 & -10.519 & 0.2109 & 0.5286 & -10.820 & -11.032 & 0.4971 & 0.9350 & -10.496 & -11.185 & 0.0329 & \\
\hline unknown & -10.311 & -10.222 & 0.5616 & 0.7959 & -10.230 & -10.134 & 0.4 & 0.9350 & -10.280 & 18 & 0.7025 & 0 \\
\hline unknown & -11.786 & -11.673 & 0.8795 & 0.9024 & -11.796 & -12.317 & 0.3719 & 0.9350 & -11.358 & -11.331 & 0.9529 & 91 \\
\hline unknown & -10.276 & -10.411 & 0.6974 & 0.8374 & -10.004 & -11.010 & 0.0324 & 0.9350 & -10.874 & -10.962 & 0.9109 & 94 \\
\hline unknown & -11.312 & -10.975 & 0.1563 & 0.4746 & -11.272 & -11.211 & 0.7950 & 0.9665 & -10.486 & -10.934 & 0.2064 & 0.48 \\
\hline unknown & -10.291 & -10.393 & 0.5323 & 0.7826 & -10.314 & -10.306 & 0.9235 & 0.9804 & -10.666 & -10.303 & 0.1141 & 0.41 \\
\hline unknown & -9.609 & -9.216 & 0.0416 & 0.3335 & -9.683 & -9.626 & 0.8656 & 0.9665 & -9.379 & -9.643 & 0.3653 & \\
\hline unknown & -8.165 & -8.224 & 0.4883 & 0.7575 & -8.379 & -8.211 & 0.1679 & 0.9350 & -8.347 & -8.283 & 0.6292 & \\
\hline unknown & -8.647 & -8.682 & 0.7869 & 0.8805 & -8.713 & -8.565 & 0.2527 & 0.9350 & -8.949 & -8.770 & 0.2429 & 0.5121 \\
\hline unknown & -10.016 & -9.627 & 0.0332 & 0.2953 & -9.809 & -9.646 & 0.5941 & 0.9350 & -9.593 & -9.778 & 0.5277 & \\
\hline unknown & -10.850 & -11.089 & 0.5893 & 0.8112 & -10.654 & -11.412 & 0.0247 & 0.9350 & -11.865 & -11.417 & 0.5182 & 0 \\
\hline unknown & -10.460 & -10.715 & 0.2558 & 0.5759 & -10.560 & -10.631 & 0.7890 & 0.9665 & -10.394 & -10.519 & 0.6747 & \\
\hline unknown & -9.165 & -9.456 & 0.2096 & 0.5278 & -9.304 & -9.938 & 0.0737 & 0.9350 & -9.978 & -9.707 & 0.6348 & 0 \\
\hline unknown & -10.011 & -10.142 & 0.7670 & 0.8735 & -9.874 & -10.647 & 0.1202 & 0.9350 & -11.469 & -10.949 & 0.5336 & 0.741 \\
\hline unknown & -10.150 & -9.886 & 0.3551 & 0.6476 & -10.042 & -9.985 & 0.8470 & 0.9665 & -9.759 & -9.935 & 0.5295 & - \\
\hline unknown & -9.148 & -8.871 & 0.2424 & 0.5588 & -8.976 & -9.226 & 0.3006 & 0.9350 & -8.787 & -9.160 & 0.0726 & \\
\hline unknown & -8.674 & -8.385 & 0.1111 & 0.4448 & -8.864 & -8.635 & 0.5263 & 0.9350 & -8.544 & -8.879 & 0.1694 & 0.44 \\
\hline unknown & -9.332 & -9.230 & 0.5285 & 0.7826 & -9.248 & -9.685 & 0.1279 & 0.9350 & -9.082 & -9.579 & 0.0519 & 0.393 \\
\hline unknown & -10.125 & -9.953 & 0.3309 & 0.6346 & -9.962 & -10.483 & 0.1358 & 0.9350 & -9.857 & -10.226 & 0.0699 & . \\
\hline unknown & -9.769 & -9.754 & 0.9160 & 0.9160 & -9.828 & -9.756 & 0.4733 & 0.9350 & -9.819 & -9.690 & 0.5280 & 7 \\
\hline unknown & -10.932 & -11.374 & 0.3755 & 0.6533 & -10.738 & -11.915 & 0.1056 & 0.9350 & -12.197 & -12.114 & 0.9170 & 0 \\
\hline unknown & -8.841 & -9.110 & 0.5572 & 0.7959 & -8.660 & -9.490 & 0.0197 & 0.9350 & -10.391 & -9.968 & 0.5652 & \\
\hline unknown & -9.872 & -9.630 & 0.6155 & 0.8186 & -9.849 & -10.035 & 0.5582 & 0.9350 & -9.508 & -9.710 & 0.5939 & \\
\hline unknown & -7.736 & -7.988 & 0.2209 & 0.5443 & -7.813 & -8.297 & 0.2581 & 0.9350 & -8.458 & -8.297 & 0.7961 & \\
\hline unknown & -8.986 & -8.886 & 0.7797 & 0.8762 & -8.903 & -9.356 & 0.1202 & 0.9350 & -8.694 & -8.989 & 0.2286 & \\
\hline unknown & -9.668 & -9.676 & 0.9846 & 0.9846 & -9.549 & -10.029 & 0.1271 & 0.9350 & -9.363 & -9.718 & 0.0774 & 0.593 \\
\hline unknown & -10.370 & -10.785 & 0.2412 & 0.5588 & -10.323 & -11.350 & 0.0046 & 0.9176 & -11.959 & -11.507 & 0.6128 & 0.801 \\
\hline unknown & -9.196 & -9.299 & 0.4423 & 0.7141 & -9.386 & -9.306 & 0.4876 & 0.9350 & -9.674 & -9.358 & 0.0353 & 0.393 \\
\hline unknown & -7.866 & -7.984 & 0.2251 & 0.5509 & -8.043 & -7.940 & 0.4642 & 0.9350 & -8.109 & -7.938 & 0.0756 & \\
\hline unknown & -9.793 & -10.071 & 0.1848 & 0.5057 & -9.599 & -10.271 & 0.0915 & 0.9350 & -10.307 & -10.581 & 0.6858 & \\
\hline unknown & -11.205 & -11.652 & 0.5615 & 0.7959 & -11.070 & -10.855 & 0.8215 & 0.9665 & -11.857 & -11.513 & 0.7586 & \\
\hline unknown & -10.645 & -11.047 & 0.3265 & 0.6346 & -10.363 & -11.291 & 0.0115 & 0.9350 & -11.716 & -11.648 & 0.9133 & \\
\hline unknown & -7.040 & -7.397 & 0.3273 & 0.6346 & -6.949 & -7.739 & 0.0421 & 0.9350 & -8.457 & -8.615 & 0.8048 & \\
\hline
\end{tabular}




\begin{tabular}{|c|c|c|c|c|c|c|c|c|c|c|c|c|}
\hline unknown & -9.280 & -9.637 & 0.1434 & 0.4623 & -9.549 & -10.037 & 0.4343 & 0.9350 & -10.345 & -10.208 & 0.8248 & 0.9103 \\
\hline unknown & -8.956 & -9.086 & 0.3203 & 0.6323 & -9.148 & -8.962 & 0.1862 & 0.9350 & -9.119 & -8.987 & 0.3154 & 0.5752 \\
\hline unknown & -10.872 & -10.974 & 0.9087 & 0.9087 & -10.237 & -10.144 & 0.9155 & 0.9804 & -10.842 & -11.023 & 0.8407 & 0.9167 \\
\hline unknown & -9.668 & -9.636 & 0.8050 & 0.8834 & -9.765 & -9.660 & 0.5259 & 0.9350 & -9.944 & -9.742 & 0.1067 & 0.4114 \\
\hline unknown & -10.903 & -10.475 & 0.0792 & 0.4121 & -10.666 & -10.367 & 0.2985 & 0.9350 & -10.283 & -10.619 & 0.2221 & 0.4934 \\
\hline unknown & -9.782 & -9.494 & 0.1971 & 0.5141 & -9.552 & -9.840 & 0.2403 & 0.9350 & -9.388 & -9.719 & 0.1228 & 0.4209 \\
\hline unknown & -9.515 & -9.273 & 0.0996 & 0.4300 & -9.724 & -9.422 & 0.3187 & 0.9350 & -9.508 & -9.682 & 0.4224 & 0.6573 \\
\hline unknown & -9.898 & -9.921 & 0.9052 & 0.9052 & -9.930 & -10.332 & 0.1569 & 0.9350 & -9.665 & -10.223 & 0.0300 & 0.3934 \\
\hline unknown & -10.601 & -10.262 & 0.4167 & 0.6953 & -10.684 & -10.793 & 0.6392 & 0.9368 & -10.225 & -10.336 & 0.7126 & 0.8499 \\
\hline unknown & -9.694 & -9.716 & 0.9456 & 0.9456 & -9.632 & -10.036 & 0.1616 & 0.9350 & -9.396 & -9.669 & 0.2066 & 0.4828 \\
\hline unknown & -10.329 & -10.143 & 0.6324 & 0.8224 & -10.304 & -10.503 & 0.3658 & 0.9350 & -9.942 & -10.384 & 0.0950 & 0.4048 \\
\hline unknown & -10.248 & -10.386 & 0.4814 & 0.7508 & -10.543 & -10.352 & 0.3168 & 0.9350 & -10.702 & -10.448 & 0.2325 & 0.5007 \\
\hline unknown & -8.973 & -9.005 & 0.7400 & 0.8557 & -9.108 & -8.994 & 0.3690 & 0.9350 & -9.215 & -9.045 & 0.1283 & 0.4215 \\
\hline unknown & -9.692 & -10.020 & 0.0048 & 0.1659 & -9.976 & -9.796 & 0.0955 & 0.9350 & -9.956 & -9.773 & 0.3652 & 0.6135 \\
\hline unknown & -10.407 & -10.080 & 0.0488 & 0.3484 & -10.490 & -10.247 & 0.4020 & 0.9350 & -10.299 & -10.559 & 0.2182 & 0.4928 \\
\hline unknown & -6.935 & -7.825 & 0.0721 & 0.4017 & -6.023 & -5.970 & 0.9141 & 0.9804 & -6.695 & -7.421 & 0.2087 & 0.4828 \\
\hline unknown & -10.657 & -10.438 & 0.1244 & 0.4520 & -10.456 & -10.708 & 0.3308 & 0.9350 & -10.240 & -10.683 & 0.0927 & 0.4048 \\
\hline unknown & -10.885 & -10.730 & 0.7227 & 0.8518 & -10.981 & -11.332 & 0.4032 & 0.9350 & -10.863 & -10.834 & 0.9217 & 0.9475 \\
\hline
\end{tabular}

${ }^{\mathrm{a}}$ The ambiguous subspecies indicates that the lipidomic profiling gave two possible identifications of the lipid. ${ }^{\mathrm{b}}$ Abbreviations: Cer, ceramide. HexCer, hexosylceramide. SM, sphingomyelin. PC, phosphatidylcholine. PE, phosphatidylethanolamine. PI, phosphatidylinositol. 
a)

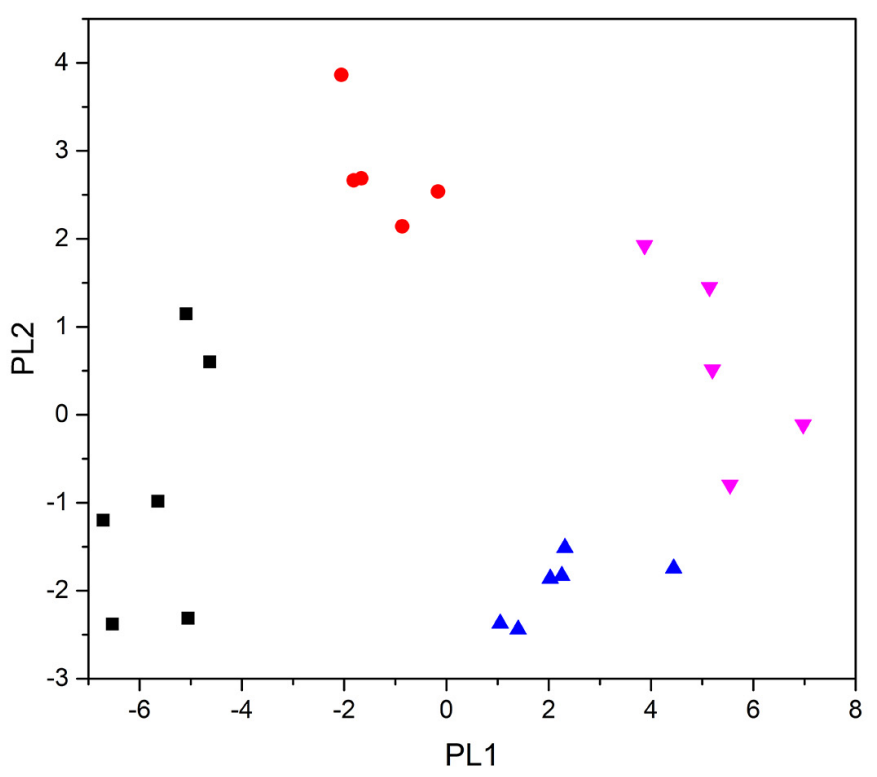

b)

Liver ESI-

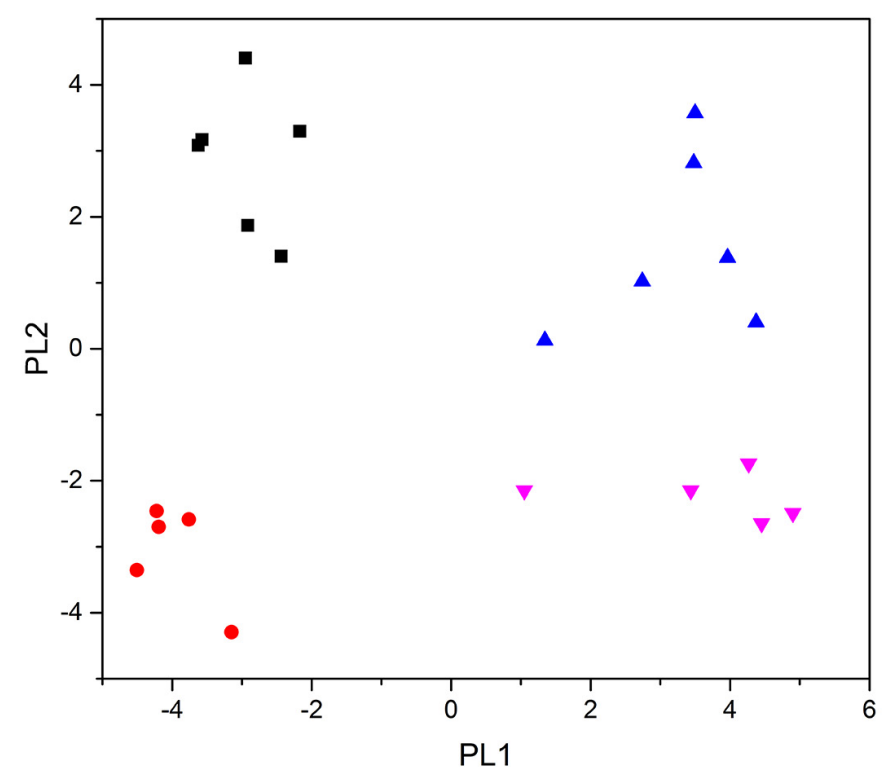

- $\mathrm{CON},-8 \mathrm{~d}-\mathrm{HIGH},-8 \mathrm{~d} \wedge \mathrm{CON}, 9 \mathrm{~d} \nabla \mathrm{HIGH}, 9 \mathrm{~d}$

\section{Online Resource Fig. S1}

Combined principal component and linear discriminant analysis on (a) liver positive electrospray ionization mode (ESI + ) dataset and (b) liver negative electrospray ionization mode (ESI-) dataset. These analyses reveal that the identified lipids distinguished the liver samples collected from different feeding groups and different time points. 
a)

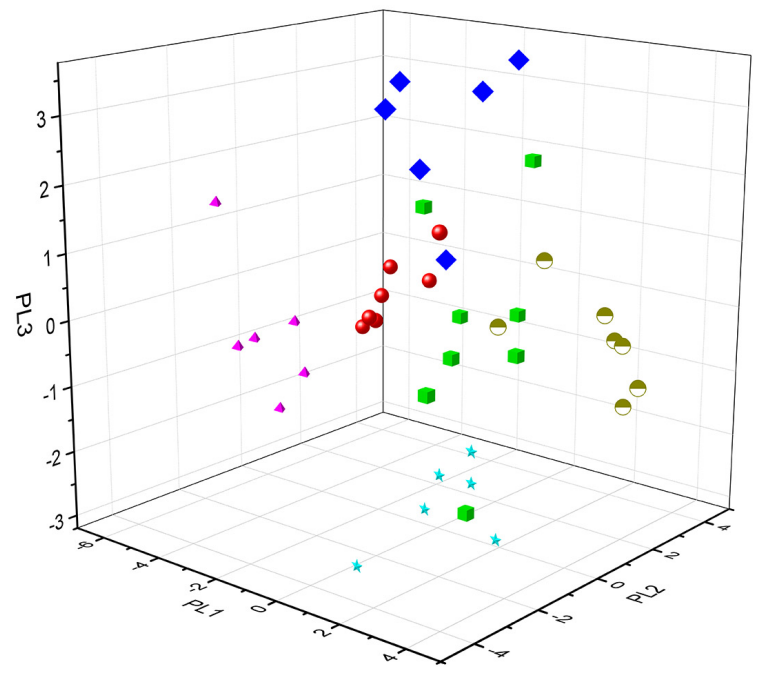

- $\operatorname{CON},-8 \mathrm{~d}=\mathrm{HIGH},-8 \mathrm{~d}$ b)

AT ESI-

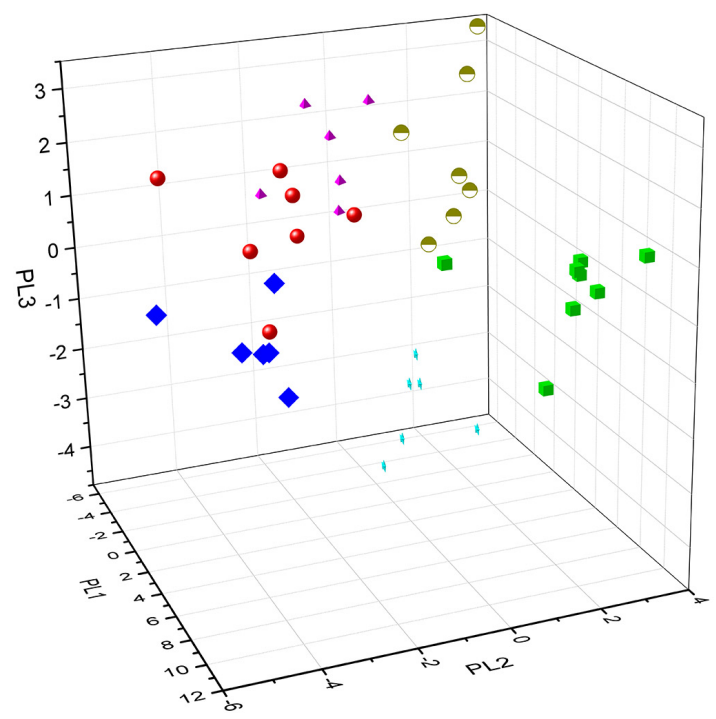

HIGH, 9d

\section{Online Resource Fig. S2}

Combined principal component and linear discriminant analysis on (a) AT positive electrospray ionization mode (ESI+) dataset and (b) AT negative electrospray ionization mode (ESI-) dataset. These analyses reveal that the identified lipids distinguished the AT samples collected from different feeding groups and different time points. 


\section{Online Resource Table S7}

Number of lipids that showed significant diet and time effects in repeated measures ANOVA.

\begin{tabular}{lcc}
\hline Dataset and effect & $\begin{array}{l}\text { Number of lipids } \\
\text { with } p<0.05 \text { in the } \\
\text { dataset }\end{array}$ & $\begin{array}{l}\text { Number of lipids with } p<0.05 \text { among the } \\
\text { 20 lipids that contribute most to the } \\
\text { separation in PCA-LDA }\end{array}$ \\
\hline Liver ESI+ Diet (PL2) & 55 & 10 \\
Liver ESI+ Day (PL1) & 179 & 14 \\
Liver ESI- Diet (PL2) & 15 & 6 \\
Liver ESI- Day (PL1) & 36 & 15 \\
AT ESI+ Diet (PL1) & 1 & 1 \\
AT ESI- Diet (PL2) & 6 & 3 \\
\hline
\end{tabular}

Abbreviations: PCA-LDA, principal component analysis-linear discriminant analysis. ESI+, positive electrospray ionization mode in mass spectrometry. ESI-, negative electrospray ionization mode in mass spectrometry. PL, PCA-LDA function. AT, adipose tissue. 